\title{
Predictions of Spectral Parameters by Several Inflationary Universe Models in Light of the Planck Results
}

\author{
Øyvind Gron \\ College of Applied Sciences, Faculty of Technology, Art and Design, Oslo and Akershus University, \\ NO-0130 Oslo, Norway; Oyvind.Gron@hioa.no
}

Received: 29 September 2017; Accepted: 5 December 2017; Published: 29 January 2018

\begin{abstract}
I give a review of predictions of values of spectral parameters for a large number of inflationary models. The present review includes detailed deductions and information about the approximations that have been made, written in a style that is suitable for text book authors. The Planck data have the power of falsifying several models of inflation as shown in the present paper. Furthermore, they fix the beginning of the inflationary era to a time about $10^{-36} \mathrm{~s}$, and the typical energy of a particle at this point of time to $10^{16} \mathrm{GeV}$, only a few orders of magnitude less than the Planck energy, and at least 12 orders of magnitude larger than the most energetic particle produced by CERN's particle accelerator, LHC. This is a phenomenological review with contents as given in the list below. It includes systematic presentations of the different types of slow roll parameters that have been in use, and also of the $\mathrm{N}$-formalism.
\end{abstract}

Keywords: cosmology; inflationary models; spectral parameters; Planck measurements

\section{Introduction}

We have a so-called standard-model for the evolution of the universe. According to this model, the universe started from a quantum fluctuation where the universe appeared in a state dominated by dark energy with extremely great density. The dark energy caused repulsive gravity and made the universe expand with great acceleration.

This state lasted for about $10^{-33} \mathrm{~s}$, and the distances between reference points then increased by 50-60 e-folds. This is called the inflationary era of the universe. At the beginning of this era, there was thermal equilibrium, which explains the observed isotropy of the CMB-temperature. Also, space inflated and became nearly flat, i.e., the geometry of the three-dimensional space became close to Euclidean, meaning that the sum of the densities of all types of cosmic energy and matter approached the critical density. This explains that the observed density is so close to the critical density.

The Big-Bang explosion that caused most of the observed expansion velocity of the universe, may have been this era. Also quantum fluctuations happened at the beginning of the inflationary era, and they were the seeds from which the structure of the universe evolved. Calculations show that these fluctuations had a scale invariant spectrum, explaining the observed Harrison-Zel'dovich spectrum of the large scale structure in the universe.

At the beginning of the inflationary era, there were wildly changing patterns in the cosmic density distribution, and these changing shapes produced gravity waves. These gravity waves functioned as messengers telling about events that happened before the universe was $10^{-35} \mathrm{~s}$ old. About 380,000 years later the gravity waves imprinted upon the CMB a B-mode polarization pattern, which then became observable when the universe became transparent for this radiation.

The possibility that the B-mode signal observed by BICEP 2 was due to galactic dust in the Milky way and not to primordial gravitational waves, was discussed early on. A preprint from the 
Planck team that came in September 2014 concluded that all of the BICEP 2 signal might be due to galactic dust [1]. They concluded that in order to clarify the consequences of the BICEP 2 and Planck observations that had been made up to then, the two teams ought to co-operate about the analysis of the observational data. A common report came in a preprint 3 February 2015 [2]. At the present time the conclusion is that the observed B-mode signal most probably is of a galactic origin.

However during the next years a more accurate mapping of the B-mode polarization contributed by galactic dust may make it possible to subtract the galactic contribution from the observed signal, and if the primordial contribution is not too small, then it may then become detectable.

In the present situation with new observations of the B-mode polarization pattern in the CMB radiation field expected the next years, the predictions of spectral parameters from different inflationary models should be presented in a way suitable for chapters in text books and for teachers and students.

In this article I will provide detailed deductions of the values of spectral parameters and of relationships between spectral parameters, for the inflationary models in the list below. Consequences of the Planck-data for the inflation models are also considered.

\begin{tabular}{|c|c|c|}
\hline Number & Name & Potential \\
\hline 1 & Polynomial chaotic inflation & $V(\phi)=M^{4} \hat{\phi}^{p}, \quad \hat{\phi}=\phi / M_{P}$ \\
\hline & & $V(\phi) \approx M^{4}\left(1-\frac{1}{2} \eta_{0} \hat{\phi}^{2}\right)$ \\
\hline 2 & Hilltop inflation & $\begin{array}{r}V(\phi) \approx V_{0}\left(1-\widetilde{\phi}^{p}\right), \quad \widetilde{\phi}=\phi / \phi_{0}, \quad p \neq 1 \quad \& \quad p \neq 2 \\
V(\phi)=\left(1-n \phi^{\alpha}\right)^{\beta}\end{array}$ \\
\hline 3 & $\begin{array}{l}\text { Symmetry breaking inflation } \\
\text { Double well inflation }\end{array}$ & $\begin{array}{c}V(\phi)=M^{4}\left(\phi^{2}-M^{2}\right)^{2} \\
V(\phi)=V_{0}\left[1-(\phi / \mu)^{2}\right]^{2}\end{array}$ \\
\hline 4 & $\begin{array}{l}\text { Exponential potential and } \\
\text { power law inflation }\end{array}$ & $\begin{aligned} V(\phi) & =M^{4} e^{-\lambda \hat{\phi}} \\
V(\phi) & =V_{0} e^{-\lambda \hat{\phi}^{p}}\end{aligned}$ \\
\hline 5 & Natural inflation & $\begin{array}{l}V_{-}(\phi)=V_{0}(1-\cos \widetilde{\phi})=2 V_{0} \sin ^{2}(\widetilde{\phi} / 2) \\
V_{+}(\phi)=V_{0}(1+\cos \widetilde{\phi})=2 V_{0} \cos ^{2}(\widetilde{\phi} / 2)\end{array}$ \\
\hline 6 & Hybrid natural inflation & $V(\phi)=V_{0}(1-\beta \cos \widetilde{\phi})$ \\
\hline 7 & Higgs-Starobinsky inflation & $\begin{array}{c}V(\phi)=\left\{\begin{array}{lc}\left(V_{0} \tilde{\xi}^{2} / M_{P}^{4}\right)\left(\hat{\phi}^{2}-v^{2}\right)^{2}, & \hat{\phi}<<1 / \xi \\
V_{0}\left(1-e^{-\sqrt{2 / 3} \hat{\phi}}\right)^{2}, & \hat{\phi}>>1 / \xi\end{array}\right. \\
V(\phi)=V_{0}\left(1+e^{-\sqrt{2 / 3} \hat{\phi}}\right)^{-2}, V(\phi)=V_{0}\left(1-e^{-q \hat{\phi}}\right), \\
V(\phi)=V_{0}\left(1-e^{-\sqrt{2 / 3 \alpha} \hat{\phi}}\right)^{2}, V(\phi)=V_{0}\left(1-e^{\alpha \hat{\phi}}\right)^{\beta}\end{array}$ \\
\hline 8 & S-dual inflation & $V(\phi)=V_{0} \cosh ^{p} \widetilde{\phi}$ \\
\hline 9 & Hyperbolic inflation & $V(\phi)=A \sinh ^{p} \widetilde{\phi}$ \\
\hline 10 & M-inflation & $V(\phi) \propto \phi^{2}(\phi-\mu)^{2}$ \\
\hline 11 & $\begin{array}{l}\text { Supergravity motivated } \\
\text { inflation }\end{array}$ & $V(\phi)=V_{0} \alpha^{p / 2} \tanh ^{p}(\phi / M), M=M_{P} \sqrt{6 \alpha}$ \\
\hline 12 & Goldstone inflation & $V(\phi)=V_{0} \cos ^{2} \hat{\phi}$ \\
\hline 13 & Coleman-Weinberg inflation & $V(\phi)=V_{0}\left\{\hat{\phi}^{4}\left[\ln \hat{\phi}-\frac{1}{4}\right]+\frac{1}{4}\right\}$ \\
\hline 14 & Kähler moduli inflation & $V(\phi)=V_{0}\left(1-\alpha \hat{\phi}^{4 / 3} e^{-\beta \hat{\phi}^{4 / 3}}\right)$ \\
\hline 15 & Hybrid inflation & $\begin{array}{c}V(\chi, \phi)=g^{2}\left(M^{2}-\frac{\chi^{2}}{4}\right)^{2}+\frac{m^{2}}{2} \phi^{2}+\frac{\lambda^{2}}{4} \chi^{2} \phi^{2} \\
V(\phi)=V_{0}\left(1+\widetilde{\phi}^{2}\right), \quad \tilde{\phi}=m \phi / \sqrt{2 V_{0}}, \quad V_{0}=g^{2} M^{4}\end{array}$ \\
\hline 16 & Brane inflation & $\begin{array}{l}\text { In this class of models the Friedmann equation takes the form } \\
\qquad H^{2}=\frac{\kappa}{3}\left[\frac{1}{2} \dot{\phi}^{2}+V\left(1+\frac{V}{2 \lambda}\right)\right]\end{array}$ \\
\hline
\end{tabular}




\begin{tabular}{|c|c|c|}
\hline Number & Name & Potential \\
\hline 17 & Fast roll inflation & $\begin{aligned} V(\phi) & =(1 / 2) M^{2} M_{P}^{2}[6+\alpha-\alpha \cosh (\sqrt{2(3+\alpha)} \hat{\phi}) \\
& =M^{2} M_{P}^{2}\left[3+\alpha-\alpha \cosh ^{2}\left(\sqrt{\frac{3+\alpha}{2}} \hat{\phi}\right)\right]\end{aligned}$ \\
\hline 18 & Running mass inflation & $V(\phi)=V_{0}\left[1-\frac{\phi^{2}}{M^{2}}\left(\ln \frac{\phi}{\phi_{0}}-\frac{1}{2}\right)\right]$ \\
\hline 19 & k-inflation & unspecified \\
\hline 20 & $\begin{array}{l}\text { Dirac-Born-Infield } \\
\text { (DBI) inflation }\end{array}$ & $V \propto \phi^{p}$ \\
\hline 21 & $\begin{array}{l}\text { Loop of flux-brane inflation } \\
\text { Spontaneously broken } \\
\text { SUSY inflation }\end{array}$ & $V(\phi)=V_{0}(1+\alpha \ln \hat{\phi})$ \\
\hline 22 & Mutated hilltop inflation & $V(\phi)=V_{0}[1-1 / \cosh (\alpha \hat{\phi})]$ \\
\hline 23 & Arctan inflation & $V(\phi)=V_{0}\left(1+\frac{2}{\pi} \arctan \bar{\phi}\right), \quad \bar{\phi}=\phi / M$ \\
\hline 24 & $\begin{array}{l}\text { Inflation with fractional } \\
\text { potential }\end{array}$ & $V(\phi)=V_{0} \frac{\alpha \hat{\phi}^{2}}{1+\alpha \hat{\phi}^{2}}$ \\
\hline 25 & Twisted inflation & $V(\phi)=M^{4}\left(1-A \widetilde{\phi}^{2} e^{-\widetilde{\phi}}\right), \quad \widetilde{\phi}=\phi / \phi_{0}$ \\
\hline 26 & $\begin{array}{l}\text { Inflation with invariant } \\
\text { density spectrum }\end{array}$ & $V(\phi)=V_{0}(1-\alpha \hat{\phi})^{-2}$ \\
\hline 27 & Quintessential inflation & $\begin{array}{c}V(\phi)=\sinh ^{2}\left(\frac{\alpha}{2} \hat{\phi}\right), \\
V=\frac{V_{0}}{2}[1+\tanh (p \hat{\phi})], \quad p>0 \\
V=M^{4} \exp \left(-2 n e^{\phi} / \sqrt{2} N_{1}\right) \\
V(\phi)=\frac{V_{0}}{\cosh \left[(\beta \hat{\phi})^{n}\right]}\end{array}$ \\
\hline 28 & $\begin{array}{l}\text { Generalized Chaplygin Gas } \\
\text { (GCG) inflation }\end{array}$ & $V(\phi)=\left(V_{0} / 2\right) \frac{1+\cosh ^{2} \breve{\phi}}{\cosh ^{2(1+3 / m)} \bar{\phi}}$ \\
\hline 29 & Axion monodromy inflation & $V=a_{1} \hat{\phi}+a_{2} \cos \left(\frac{\hat{\phi}}{f}+\delta\right)$ \\
\hline 30 & $\begin{array}{c}\text { Intermediate } \\
\text { inflationBrane-intermediate } \\
\text { inflation }\end{array}$ & $a(t)=a_{0} e^{A\left(M_{P} t\right)^{\alpha}} \quad, \quad 0<\alpha<1$ \\
\hline 31 & Constant-roll inflation & $\eta_{H}=$ constant \\
\hline 32 & Warm inflation & Dissipation of inflaton energy to radiation \\
\hline 33 & Tachyon inflation & $V(T)=V_{0}^{\frac{2 \beta}{1-2 \beta}} T^{\frac{4 \beta}{1-2 \beta}} \quad, \quad \beta \neq 1 / 2, \phi=\sqrt{V} T$ \\
\hline
\end{tabular}

The present review is different from earlier ones in several ways. I. It is focused upon predicted values of the scalar spectral index and the tensor-to-scalar-ratio for a large number of inflationary models. II. The presentation is self contained to a larger degree than usual, like a text book. III. Also, it includes in between calculations and details of the deductions to a larger degree than usual. IV. There are systematic and detailed presentations of the three main types of slow roll parameters that have been used to describe inflationary universe models, and the relationships between these parameters. V. Also, I give an encompassing review of the $N$-formalism with applications to a large number of inflationary universe models. VI. The large classes of warm and tachyonic inflationary universe models are thoroughly reviewed.

\section{The Inflationary Era of the Universe}

The inflationary era of the universe was an extremely brief period lasting only for $10^{-33} \mathrm{~s}$ with approximately exponentially accelerating expansion of the universe [3-5]. It is usually assumed that the inflaton field had a large value before inflation and rolled down the potential during inflation. 
Before the announcement of the BICEP2 results we did not know when the inflationary era started. The earlier it started the warmer it was, and the larger was the energy per particle. At the Planck time $t_{P}=\sqrt{G h / c^{5}}=1.4 \times 10^{-43} \mathrm{~s}$ the energy per particle was equal to the Planck energy, $E_{P}=\sqrt{h c^{5} / G}=1.2 \times 10^{19} \mathrm{GeV}$, where $h=6.6 \times 10^{34} \mathrm{Js}$ is Planck's constant. In this connection the energy $E$ of the inflationary era is said to be small if $E<<E_{P}$.

Comment on notation. Einstein's gravitational constant is $\kappa=8 \pi \mathrm{G} / \mathrm{c}^{4}$. The reduced Planck mass is often defined as $M_{P}=\sqrt{h / \kappa c}=4.3 \times 10^{-9} \mathrm{~kg}$ corresponding to the energy $2.4 \times 10^{18} \mathrm{GeV}$. Using units so that the velocity of light in empty space and Planck's constant $h=c=1$, Einstein's gravitational constant is $\kappa=1 / M_{P}^{2}$. In many articles one uses units so that $\kappa=1$, but we shall keep $\kappa$ or $M_{P}$ in the equations here. I will use a hat to denote that a symbol represents the relationship between a physical quantity and the corresponding Planck unit, hence it is dimensionless. For example the dimensionless quantities representing the inflaton field and time are and where is the Planck time.

One often distinguishes between large field and small field inflation. These terms concern the energy contents of the inflaton field. Large field inflation is when $\phi>M_{P}$ and small field inflation when $\phi<M_{P}$.

\section{The Dynamical Equations}

During the inflationary era the evolution of the universe is assumed to be dominated by a scalar field $\phi$ which is called the inflaton field. This field is often described as a perfect fluid with density and pressure

$$
\rho=\frac{1}{2} \dot{\phi}^{2}+V, \quad p=\frac{1}{2} \dot{\phi}^{2}-V .
$$

The first Friedmann equation is

$$
H^{2}=\frac{\kappa}{3} \rho=\frac{\kappa}{3}\left(\frac{1}{2} \dot{\phi}^{2}+V\right)
$$

where the dot denotes differentiation with respect to cosmic time, $H=\dot{a} / a$ is the Hubble parameter that is assumed to be positive (expansion), $\rho$ is the energy density of the inflaton field, and $V=V(\phi)$ is the potential of the inflaton field. The continuity equation is

$$
\dot{\rho}+3 H(\rho+p)=0
$$

Differentiating the first of the Equation (2.1) with respect to time and using that $\dot{V}=V^{\prime} \dot{\phi}$, where $V^{\prime}=d V / d \phi$ and $\dot{V}=d V / d t$, we obtain $\dot{\rho}=\dot{\phi}\left(\ddot{\phi}+V^{\prime}\right)$

$$
\dot{\rho}=\dot{\phi}\left(\ddot{\phi}+V^{\prime}\right) \text {. }
$$

Inserting this and $\rho+p=\dot{\phi}^{2}$ from Equation (2.1) into the continuity Equation (2.3) we get the evolution equation for the inflaton field,

$$
\ddot{\phi}+3 H \dot{\phi}+V^{\prime}=0 \text {. }
$$

This equation shows that a constant inflaton field requires a flat scalar potential, $V^{\prime}=0$. For a flat scalar potential, on the other hand, integration of Equation (2.5) gives

$$
a^{3} \dot{\phi}=K,
$$

where $K$ is a non-negative constant. Hence the inflaton field is either constant or increases with time if the potential is flat. 
It follows from the second Friedmann equation that the acceleration of the cosmic expansion is given by

$$
\frac{\ddot{a}}{a}=-\frac{\kappa}{6}(\rho+3 p)
$$

The inflaton field is often described as a perfect fluid with density and pressure as given in Equation (2.1). Hence, the fluid obeys the equation of state

$$
p=w \rho, \quad w=\frac{(1 / 2) \dot{\phi}^{2}-V}{(1 / 2) \dot{\phi}^{2}+V} .
$$

For $-1<w<1$ the inflaton field interpolates between a Lorentz invariant vacuum energy (LIVE) with $w=-1$ for a constant inflaton field and a Zel'dovich fluid with $w=1$ for a flat potential with $V=0$. Solved with respect to $V$ the second of these equations gives

$$
V=\frac{1}{2} \frac{1-w}{1+w} \dot{\phi}^{2}
$$

showing that $V>0$ for $|w|<1$.

Using Equations (2.2) and (2.8) the acceleration Equation (2.7) of the scale factor takes the form

$$
\ddot{a}=-\frac{a H^{2}}{2}(1+3 w) .
$$

With Equation (2.1) the same equation may be written as

$$
\frac{\ddot{a}}{a}=-\frac{\kappa}{3}\left(\dot{\phi}^{2}-V\right)
$$

Hence accelerated expansion requires that $V>\dot{\phi}^{2}$ or, from Equation (2.10), that $w<-1 / 3$. Differentiating Equation (2.2), inserting Equation (2.5) and using that $V^{\prime} \dot{\phi}=\dot{V}$ gives

$$
\dot{H}=-(\kappa / 2) \dot{\phi}^{2},
$$

or

$$
\dot{\phi}=-(2 / \kappa) H^{\prime},
$$

where $H^{\prime}=d H / d \phi=\dot{H} / \dot{\phi}>0$ since $\dot{H}<0$ according to Equation (2.12), and $\dot{\phi}<0$ because the inflaton field rolls down the potential. It follows from Equations (2.2) and (2.13) that

$$
\kappa^{2} V=3 \kappa H^{2}-2 H^{\prime 2} .
$$

Equation (2.12) shows that the Hubble parameter is constant and there is exponential expansion for a constant inflaton field. This represents the case where the inflaton field behaves like Lorentz invariant vacuum energy (LIVE) with a constant density, which may be represented by a cosmological constant. Equation (2.12) implies that the Hubble parameter is a decreasing function of time for a variable scalar field.

During most of the inflationary era, i.e., except during the transient phases at the beginning and the end of the era, the scalar field changes very slowly so that $\ddot{\phi}<<H \dot{\phi}$. Then Equation (2.5) reduces to

$$
3 H \dot{\phi}+V^{\prime}=0 .
$$


If the potential $V$ is not too small, the condition $\dot{\phi}^{2}<<V$ may also be satisfied. Then Equations (2.2), (2.8) and (2.14) reduce to

$$
\kappa V \approx 3 H^{2}, \quad w \approx-1,
$$

which means that the inflaton field behaves like LIVE with approximately constant energy density, and with exponential expansion of the space during most of the inflationary era. It follows from Equations (2.15) and (2.16) that

$$
\dot{\phi}=-\frac{2}{\sqrt{3 \kappa}}\left(V^{1 / 2}\right)^{\prime} \text {. }
$$

Equations (2.9) and (2.12) give

$$
\dot{H}=-\kappa V \frac{1+w}{1-w} .
$$

It follows from Equations (2.2) and (2.12) that

$$
\kappa V=\dot{H}+3 H^{2} .
$$

Hence

$$
\dot{H}=-(3 / 2)(1+w) H^{2} .
$$

This equation is exact. In general, i.e., for most inflation models, the equation of state parameter $w$ is not constant. However in the special case with constant $w \neq-1$ integration of Equation (2.20) gives

$$
a=a_{1}\left(\frac{t}{t_{1}}\right)^{\frac{2}{3(1+w)}} .
$$

Hence, power law expansion corresponds to a constant equation of state parameter $w \neq-1$ during the inflationary era, and exponential expansion to $w=-1$. Inserting the first of Equation (2.8) into Equation (2.3) gives

$$
\dot{\rho}=-\sqrt{3 \kappa \rho}(1+w) \rho .
$$

Integrating this equation for $w \neq-1$ with $\rho(0)=\rho_{0}$ leads to

$$
\rho=\frac{\rho_{0}}{\left[1+(1 / 2)(1+w) \sqrt{3 \kappa \rho_{0}} t\right]^{2}} .
$$

Hence for $\sqrt{\rho_{0}} t>>M_{P}$ the energy density of an inflaton field with constant equation of state parameter $w \neq-1$ decreases approximately inversely proportionally to the square of time. As shown by Equation (2.22) the density is constant if $w=-1$.

In the case of a flat potential Equations (2.6) and (2.21) give

$$
\dot{\phi}=K_{1} t^{-\frac{2}{1+w}},
$$

where $K_{1}$ is a positive constant. Integration leads to

$$
\phi=K_{2}-K_{1} \frac{1+w}{1-w} t^{-(1-w) /(1+w)} .
$$

In this case the inflaton field increases for all values of $p$. For $p>0$, i.e., for $-1<w<1$ the inflaton field then approaches the constant value $K_{2}$ for large values of $t$. 


\section{The Slow Roll Parameters}

In the theory of the inflationary universe models three different types of slow roll parameters have been commonly in use. The first set of parameters is defined in terms of the derivatives of the potential with respect to the inflaton field. They may be called the potential slow roll parameters.

\subsection{The Potential Slow Roll Parameters}

The standard definitions of the potential slow roll parameters are

$$
\varepsilon \equiv \frac{1}{2 \kappa}\left(\frac{V^{\prime}}{V}\right)^{2}, \quad \eta \equiv \frac{1}{\kappa} \frac{V^{\prime \prime}}{V}, \quad \xi \equiv \frac{1}{\kappa^{2}} \frac{V^{\prime} V^{\prime \prime \prime}}{V^{2}} \quad, \quad \sigma \equiv \frac{1}{\kappa^{3}} \frac{V^{\prime 2} V^{\prime \prime \prime \prime}}{V^{3}} .
$$

It is usual to write $\xi^{2}$ instead of $\xi$ in the third expression, but we will not put any restriction upon the sign of $V^{\prime} V^{\prime \prime \prime}$ here. The absolute values of the slow roll parameters are much less than one during the slow roll period. This means that during a slow-roll period the graph of $V(\phi)$ is very flat and has small curvature.

If $\varepsilon=$ constant, integration of the first Equation (3.1) gives

$$
V=V_{0} e^{\sqrt{2 \varepsilon} \hat{\phi}}
$$

In this case $\eta=2 \varepsilon, \xi=\eta^{2}, \sigma=\eta^{3}$. This represents power law inflation with an exponential potential which will be considered later in relation to the Planck observations $[1,6,7]$.

If $\eta=$ constant integration of the second Equation (3.1) gives

$$
V=V_{0} \sinh \left(\sqrt{\eta} \hat{\phi}+\hat{\phi}_{0}\right) .
$$

This corresponds to hyperbolic inflation which will be considered in Section 6.9.

In the slow roll approximation we shall assume that $\ddot{\phi}<<H \dot{\phi}$. From Equations (2.5), (2.16) and (3.1) we then get

$$
\dot{\phi}^{2} \approx \frac{V^{\prime 2}}{9 H^{2}} \approx \frac{V^{\prime 2}}{3 \kappa V}=\frac{2}{3} \varepsilon V
$$

Hence the term $(1 / 2) \dot{\phi}^{2}$ in Equation (2.1) and $\dot{\phi}^{2}$ in Equation (2.8) can be neglected in the slow roll era, giving $\rho \approx V, p \approx-V$. Thus, with a positive potential the inflaton field has negative pressure giving a repulsive gravitational contribution to the cosmic acceleration (2.7), which according to Equation (2.11) is $\ddot{a} \approx(\kappa / 3) a V$.

With the present accuracy of the measurements of the optical parameters and that expected in the coming years, it is sufficient to perform the calculations of the optical parameters for different inflationary models to first order in the slow roll parameters. Hence we are not discussing second order corrections here.

We shall later need the derivatives of the slow roll parameters with respect to the inflaton field. They can be expressed as

$$
\sqrt{\frac{2 \varepsilon}{\kappa}} \varepsilon^{\prime}=2 \varepsilon(\eta-2 \varepsilon), \quad \sqrt{\frac{2 \varepsilon}{\kappa}} \eta^{\prime}=\xi-2 \varepsilon \eta, \quad \sqrt{\frac{2 \varepsilon}{\kappa}} \xi^{\prime}=\sigma-(4 \varepsilon-\eta) \xi .
$$

The second derivatives of $\varepsilon$ and $\eta$ are [8]

$$
\varepsilon^{\prime \prime}=\kappa\left[\xi-2 \eta \varepsilon-4 \varepsilon(\eta-2 \varepsilon)+(\eta-2 \varepsilon)^{2}\right] \quad, \quad \eta^{\prime \prime}=\kappa\left[\frac{\sigma}{2 \varepsilon}+\eta(4 \varepsilon-\eta)-2 \xi\right] .
$$




\subsection{The Hubble Slow Roll Parameters}

Secondly, one defines Hubble slow roll parameters, $\varepsilon_{H}, \eta_{H}$ and $\xi_{H}$, in terms of the Hubble parameter and its derivatives with respect to the inflaton field $[9,10]$,

$$
\varepsilon_{H}=\frac{2}{\kappa}\left(\frac{H^{\prime}}{H}\right)^{2} \quad, \quad \eta_{H}=\frac{2}{\kappa} \frac{H^{\prime \prime}}{H} \quad, \quad \xi_{H}=\frac{4}{\kappa^{2}} \frac{H^{\prime} H^{\prime \prime \prime}}{H^{2}} .
$$

Since $H^{\prime}>0$ it follows from the first of these expressions that

$$
H^{\prime}=H \sqrt{\frac{\kappa \varepsilon_{H}}{2}}
$$

nserting the first of the expressions (3.7) into Equation (2.14) we get for the inflaton potential

$$
\kappa V=\left(3-\varepsilon_{H}\right) H^{2} .
$$

It follows from Equations (2.13) and (3.8) that during the slow roll era differentiation with respect to time and with respect to the inflaton field are related by

$$
\frac{d}{d t}=-\frac{2}{\kappa} H^{\prime} \frac{d}{d \phi}=-\sqrt{\frac{2 \varepsilon_{H}}{\kappa}} H \frac{d}{d \phi}
$$

Hence

$$
H^{\prime 2}=-\frac{\kappa}{2} \dot{H} \quad, \quad H^{\prime \prime}=-\frac{\kappa}{4} \frac{\ddot{H}}{\dot{H}} \quad, \quad H^{\prime} H^{\prime \prime \prime}=\frac{\kappa^{2}}{8}\left(\frac{\ddot{H}}{\dot{H}}\right) .
$$

sing this in the definitions (3.7) we get simple expressions for $\varepsilon_{H}, \eta_{H}$ and $\xi_{H}$,

$$
\varepsilon_{H} \equiv-\frac{\dot{H}}{H^{2}} \quad, \quad \eta_{H}=-\frac{1}{2} \frac{\ddot{H}}{H \dot{H}} \quad, \quad \xi_{H}=\frac{\dddot{H}}{2 H^{2} \dot{H}}-2 \eta_{H}^{2} .
$$

$\mathrm{t}$ may be noted that $\varepsilon_{H}=1+q$, where $q$ is the decelation parameter. The expression for $\eta_{H}$ may be written

$$
\eta_{H}=\varepsilon_{H}-\frac{\dot{\varepsilon}_{H}}{2 H \varepsilon_{H}} .
$$

Since $H=\dot{a} / a$ the first Equation (3.12) takes the form

$$
\varepsilon_{H}=1-\frac{\ddot{a}}{\dot{a}^{2}} \quad \text { or } \quad \frac{\ddot{a}}{a}=H^{2}\left(1-\varepsilon_{H}\right) .
$$

A requirement for inflation is that there is accelerated expansion, $\ddot{a}>0$. Hence a necessary condition for inflation is that $\varepsilon_{H}<1$. Schwarz and Terrero-Escalante [11] have defined graceful exit from the inflationary era as the moment when $\varepsilon_{H}$ crosses unity.

It follows from Equation (2.12) that

$$
\frac{\ddot{\phi}}{\dot{\phi}}=\frac{1}{2} \frac{\ddot{H}}{\dot{H}} .
$$

Hence

$$
\varepsilon_{H}=\frac{\kappa}{2}\left(\frac{\dot{\phi}}{H}\right)^{2}, \quad \eta_{H}=-\frac{\ddot{\phi}}{H \dot{\phi}} \quad, \quad \xi_{H}=\frac{\dddot{\phi}}{H^{2} \dot{\phi}}-\eta_{H}^{2} .
$$


The equation for $\eta_{H}$ may be written

$$
\left[(1 / 2) \dot{\phi}^{2}\right]=-\eta_{H} H \dot{\phi}^{2}
$$

Hence the sign of the parameter $\eta_{H}$ decides whether the kinetic energy of the inflaton field increases, $\eta_{H}<0$, or decreases, $\eta_{H}>0$. The kinetic energy is constant for $\eta_{H}=0$.

It may be noted that the slow roll approximation should not be applied uncritically when calculating $\eta_{H}$. Inserting for $H \dot{\phi}$ from Equation (2.5) into Equation (3.16) gives

$$
\eta_{H}=\frac{3 \ddot{\phi}}{\ddot{\phi}+V^{\prime}} \text {. }
$$

Hence if the term with $\dot{\phi}^{2}$ is neglected in Equation (2.1) meaning that $\ddot{\phi} \approx 0$, one obtains $\eta_{H} \approx 0$.

There is a simple relationship between $\varepsilon, \varepsilon_{H}$ and $\eta_{H}$. Inserting the expression (2.1) for $V$ and (2.4) for $V^{\prime}$ into the expression (3.1) for $\varepsilon$ we get

$$
\varepsilon=\frac{1}{2 \kappa}\left(\frac{3 H \dot{\phi}+\ddot{\phi}}{(3 / \kappa) H^{2}-(1 / 2) \dot{\phi}^{2}}\right)^{2} .
$$

Using this together with Equations (3.13), (3.16) and (3.1) leads to

$$
\varepsilon=\varepsilon_{H}\left(\frac{3-\eta_{H}}{3-\varepsilon_{H}}\right)^{2}
$$

This relationship is exact and does not depend upon the slow roll approximation. Often $\varepsilon_{H} \approx \varepsilon$ will be a good approximation. Differentiating the slow roll Equation (2.16) gives

$$
\frac{V^{\prime}}{V}=2 \frac{H^{\prime \prime}}{H}+2\left(\frac{H^{\prime}}{H}\right)^{2}
$$

From this equation together with Equations (3.1) and (3.7) it follows that

$$
\eta=\eta_{H}+\varepsilon_{H}
$$

which is a slow roll relationship. The corresponding exact expressions for $\eta$ and $\xi$ are $[9,12,13]$

$$
\begin{aligned}
& \eta=\frac{3\left(\varepsilon_{H}+\eta_{H}\right)-\eta_{H}^{2}-\xi_{H}}{3-\varepsilon_{H}}, \\
& \xi=3 \frac{3-\eta_{H}}{\left(3-\varepsilon_{H}\right)^{2}}\left(3 \varepsilon_{H} \eta_{H}+\xi_{H}\left(1-\eta_{H}\right)-\frac{1}{6} \sigma_{H}\right), \quad \sigma_{H}=4 M_{P}^{4} \varepsilon_{H} \frac{H^{\prime \prime \prime \prime}}{H} .
\end{aligned}
$$

To lowest order this gives

$$
\xi \approx \xi_{H}+3 \varepsilon_{H} \eta_{H}
$$

In the slow roll approximation the inverse relationships are

$$
\varepsilon_{H}=\varepsilon-\frac{4}{3} \varepsilon^{2}+\frac{2}{3} \varepsilon \eta \quad, \quad \eta_{H}=(\eta-\varepsilon)\left(1-\frac{8}{3} \varepsilon\right)+\frac{1}{3}\left(\xi+\eta^{2}\right) \quad, \quad \xi_{H}=\xi-3 \varepsilon(\eta-\varepsilon) .
$$

Hence to lowest order

$$
\varepsilon_{H}=\varepsilon=\frac{1}{2 \kappa} \frac{V^{\prime 2}}{V^{2}} \quad, \quad \eta_{H}=\eta-\varepsilon=\frac{1}{\kappa}\left(\frac{V^{\prime \prime}}{V}-\frac{1}{2} \frac{V^{\prime 2}}{V^{2}}\right) .
$$


From Equations (3.5), (3.20), and (3.22) we get

$$
\varepsilon_{H}^{\prime}=\sqrt{2 \kappa \varepsilon_{H}}\left(\eta_{H}-\varepsilon_{H}\right), \quad \eta_{H}^{\prime}=\sqrt{\frac{\kappa}{2 \varepsilon_{H}}}\left(\xi_{H}-\varepsilon_{H} \eta_{H}\right) .
$$

Using Equation (3.10) we then have

$$
\dot{\varepsilon}_{H}=2 H \varepsilon_{H}\left(\varepsilon_{H}-\eta_{H}\right) \quad, \quad \dot{\eta}_{H}=H\left(\varepsilon_{H} \eta_{H}-\xi_{H}\right) .
$$

Differentiating Equation (3.9) and using Equations (3.28) and (3.12) gives

$$
\dot{V}=-H \dot{\phi}^{2}\left(3-\eta_{H}\right) \text {. }
$$

Normally $\left|\eta_{H}\right|<1$, and then the inflaton potential is a decreasing function of time. However, the potential is constant if $\eta_{H}=3$. According to Equation (3.12) this gives

$$
\ddot{H}+6 H \dot{H}=0 .
$$

Solving this equation with $H(0)=H_{0}$ leads to

$$
H(t)=H_{0} \tanh \left[3 H_{0}\left(t-t_{0}\right)\right] .
$$

As seen from Equation (3.26) $\eta_{H}=3$ corresponds to $\eta-\varepsilon=3$, or

$$
\frac{V^{\prime \prime}}{V}-\frac{1}{2} \frac{V^{\prime 2}}{V^{2}}=\frac{3}{M_{P}^{2}}
$$

The general solution of this equation is

$$
V(\phi)=[A \sinh (\sqrt{3 / 2} \hat{\phi})+B \cosh (\sqrt{3 / 2} \hat{\phi})]^{2}
$$

It should be noted that the relationships (3.26) are not exact. They are only valid in the slow roll approximation. Hence Equation (3.32) and its solution is not generally valid. Equation (3.18), however, is exact, and inserting $\eta_{H}=3$ into this equation implies $V^{\prime}=0$ or $V(\phi)=$ constant.

We further have

$$
\frac{\ddot{a}}{a}=H^{2}+\dot{H}=H^{2}\left(1+\frac{\dot{H}}{H^{2}}\right)=H^{2}\left(1-\varepsilon_{H}\right)
$$

Integration of this equation or the first of the Equation (3.12) with a constant value of $\varepsilon_{H}$ gives

$$
a= \begin{cases}K_{1}\left(\varepsilon_{H} t+K\right)^{-1 / \varepsilon_{H}}, & \varepsilon_{H} \neq 0, \\ a_{0} e^{H_{0} t} & , \quad \varepsilon_{H}=0 .\end{cases}
$$

where $K_{1}$ and $K$ are constants of integration. If $\varepsilon_{H} t<<K$ during the slow roll period, $H$ will be approximately constant. Then there will be approximately exponential expansion.

Equations (2.10) and (3.14) give

$$
\varepsilon_{H}=(3 / 2)(1+w)
$$

or

$$
w=-1+(2 / 3) \varepsilon_{H}
$$


Hence a universe with $\varepsilon_{H}=0$ is dominated by a Lorentz invariant vacuum energy (LIVE) with equation of state parameter $w=-1$ and a constant energy density which can be represented by a cosmological constant.

Inserting Equations (2.11) and (2.2) into Equation (3.14) we get

$$
\varepsilon_{H}=3 \frac{\dot{\phi}^{2} / 2}{\dot{\phi}^{2} / 2+V} .
$$

Hence the parameter $\varepsilon_{H}$ represents 3 times the ratio of the kinetic energy and the total energy of the inflaton field. This is exact. It does not require the slow roll approximation. From Equation (3.38) is seen that the condition $\varepsilon_{H}<<1$ means that the kinetic energy of the inflaton field is much smaller than its potential energy.

In the slow roll approximation Equations (2.2) and (2.5) reduce, respectively, to (2.15) and

$$
V^{\prime} \approx-3 H \dot{\phi}
$$

Hence

$$
V^{\prime \prime}=\kappa \eta V \approx 3 \eta H^{2} .
$$

Inserting Equations (3.39) and (3.40) into Equation (3.21) gives

$$
\ddot{\phi} \approx H(\varepsilon-\eta) \dot{\phi} .
$$

This equation has an interesting consequence. In the slow roll approximation we neglect $\ddot{\phi}$ in Equation (2.4), and Equation (3.41) is a slow roll version of Equation (2.4). Hence we expect that $\ddot{\phi} \approx 0$ in the slow roll era. According to Equation (3.41) this means $\varepsilon \approx \eta$ which corresponds to

$$
2 V V^{\prime \prime}=V^{\prime 2}
$$

Solving this equation with $V(0)=0$ gives

$$
V(\phi)=K \phi^{2}
$$

where $K$ is a constant of integration. This so called chaotic inflation model with a quadratic potential will be discussed later. Note that this result appears in a model independent way, only as a result of the slow roll approximation. Hence most of the inflationary models are not of a strictly slow roll type.

The end of the slow roll era is sometimes defined by the condition $\varepsilon=1$ and sometimes by $\varepsilon_{H}=1$. Let us consider the latter case. Then Equation (3.14) gives $a(t) \propto t$. This scale factor is the same as that of the Milne universe, i.e., of the Minkowski spacetime as described in an expanding reference frame. The reason for this seemingly strange relationship is found by considering Equations (2.8) and (2.11). With $\ddot{a}=0$ Equation (2.11) gives $\dot{\phi}^{2}=V$. Inserting this into Equation (2.8) shows that this particular inflaton field acts as a perfect fluid with equation of state

$$
p=-(1 / 3) \rho
$$

As seen from Equation (2.7) the gravitational mass density of this inflaton field vanishes. Media with this property are sometimes called a K-fluid [14] and sometimes a texture gas [15,16]. They do not gravitate, and this is the reason for the Milne type scale factor. 


\subsection{The Number of e-Folds}

The ratio of the final value $a_{f}$ of the scale factor during the inflationary era and the initial value $a(N)$ is

$$
\frac{a_{f}}{a(N)}=e^{N},
$$

where $N$ is called the number of e-folds of the slow roll era. Hence

$$
N=\ln \left(a_{f} / a\right) .
$$

Note that $N=0$ at the end of inflation, so that $N$ counts the number of e-folds until inflation ends and increases as we go backward in time. This is the usual choice, but some authors (for example Leach et al. [17] and Martin [18]) use $a_{i}$ where is the initial value of the scale factor during the slow roll era. We shall keep to the definition (3.45) in this article. It follows that

$$
\dot{N}=-H,
$$

or

$$
\frac{d}{d N}=-\frac{1}{H} \frac{d}{d t}
$$

Equation (2.16) implies

$$
\frac{V^{\prime}}{V}=2 \frac{H^{\prime}}{H}, V^{\prime \prime}=(6 / \kappa)\left(H^{\prime 2}+H H^{\prime \prime}\right)
$$

Using this together with Equations (3.48), (2.12), (3.49) and (3.1) and $\dot{N}=N^{\prime} \dot{\phi}$, we have

$$
d N=-\frac{H}{\dot{\phi}} d \phi=\frac{\kappa}{2} \frac{H}{H^{\prime}} d \phi=\kappa \frac{V}{V^{\prime}} d \phi=\sqrt{\frac{\kappa}{2 \varepsilon}} d \phi .
$$

This equation can be used to relate the derivative with respect to $N$ and the derivative with respect to $\phi$ as

$$
\frac{d}{d N}=\sqrt{\frac{2 \varepsilon}{\kappa}} \frac{d}{d \phi},
$$

which may be written

$$
\varepsilon=\frac{\kappa}{2} \phi_{\prime N}^{2},
$$

showing that $\varepsilon>0$. We use the notation, ${ }_{N}$ for differentiation with respect to $N$. From Equations (3.7) and (3.51) and the approximation $\varepsilon_{H} \approx \varepsilon$ we have

$$
\varepsilon_{H} \approx \frac{H, N}{H} .
$$

It follows from Equation (3.53) that $H_{, N}>0$. Differentiating this equation gives

$$
\frac{H, N N}{H, N} \approx \varepsilon_{H}+\frac{\varepsilon_{H, N}}{\varepsilon_{H}} .
$$

Also, from the definition (3.1) and Equation (3.51) we get

$$
\varepsilon=\frac{1}{2} \frac{V, N}{V} .
$$

This shows that $V, N>0$. Since $N$ increases backwards in time, this means that $V$ decreases with time in the slow roll era. 
It follows from Equations (3.52) and (3.55) that

$$
[\ln V(N)]_{N}=\kappa \phi_{, N}^{2} .
$$

From the definition (3.1), Equations (3.51), (3.49) and (3.55) we obtain

$$
\eta=2 \varepsilon+\frac{\varepsilon, N}{2 \varepsilon} \quad, \quad \xi=2 \varepsilon \eta+\eta_{, N}=4 \varepsilon^{2}+\varepsilon,,_{N}+\eta_{, N} .
$$

Using Equation (3.51) we can write Equation (3.5) as

$$
\varepsilon,_{N}=2 \varepsilon(\eta-2 \varepsilon) \quad, \quad \eta_{, N}=\xi-2 \varepsilon \eta \quad, \quad \xi_{N_{N}}=\sigma-(4 \varepsilon-\eta) \xi .
$$

The first two equations are identical to those in Equation (3.57) which has been deduced in a different way. Inserting $\varepsilon \approx \varepsilon_{H}$ and (3.22) into Equation (3.58) we obtain [19,20]

$$
\varepsilon_{H, N}=2 \varepsilon_{H}\left(\eta_{H}-\varepsilon_{H}\right) \quad, \quad \eta_{H, N}=\xi_{H}-\varepsilon_{H} \eta_{H},
$$

where the first equation is in agreement with Equation (A10) of Peiris et al. [10].

Integration of Equation (3.50) between the value of $\phi$ when the CMB scale cross the horizon, which will be our definition of the beginning of the slow roll era, and the final value $\phi_{f}$ of the inflaton field during the slow roll era, gives

$$
N \approx \kappa \int_{\phi_{f}}^{\phi} \frac{V}{V^{\prime}} d \phi=\int_{\phi_{f}}^{\phi} \sqrt{\frac{\kappa}{2 \varepsilon}} d \phi<\sqrt{\frac{\kappa}{2 \varepsilon_{\min }}}\left(\phi-\phi_{f}\right) .
$$

Note that if $V^{\prime}>0$ we must have $\phi_{f}<\phi$ in order that $N>0$, and if $V^{\prime}<0$ we must have $\phi_{f}>\phi$. Equation (3.60) implies a bound on the change of the value of the scalar field during the inflationary era,

$$
\Delta \phi>N \sqrt{\frac{2 \varepsilon_{\min }}{\kappa}}=N M_{P} \sqrt{2 \varepsilon_{\min }} .
$$

This is a first form of the so-called Lyth bound [21-24], which we shall come back to below.

Note also from Equation (3.50) that the number of e-folds is given by

$$
N=\int_{t_{*}}^{t_{f}} H d t
$$

where $t_{*}$ is the initial point of time of the slow roll era, and $t_{f}$ the final point of time which is usually defined by $\varepsilon\left(t_{f}\right)=1$.

\subsection{The Horizon-Flow Slow Roll Parameters}

There exists a third type of slow roll parameters. They have been called the horizon-flow parameters by Schwarz [11], but were called the Hubble flow parameters (or functions) by Coone et al. [25] and Martin [18]. They are defined by

$$
\varepsilon_{1}=\varepsilon_{H} \quad, \quad \varepsilon_{n+1}=-\left(\ln \left|\varepsilon_{n}\right|\right)_{N} .
$$

The minus signs are not present in the definitions of Liddle et al. [9] and Leach et al. [17], but they have used the opposite sign of the usual one in their definition of the number of $\mathrm{N}$-folds. Therefore the 
minus sign is included here in order to have the same definition of the slow roll parameters $\varepsilon_{n}$ as they have. Using Equation (3.48) we have

$$
\dot{\varepsilon}_{n}=H \varepsilon_{n} \varepsilon_{n+1},
$$

or

$$
\varepsilon_{n, N}=-\varepsilon_{n} \varepsilon_{n+1} .
$$

From Equations (3.64), (3.12) and (3.28) we find

$$
\varepsilon_{2}=\frac{\dot{\varepsilon}_{1}}{H \varepsilon_{1}}=\frac{\ddot{H}}{H \dot{H}}-2 \frac{\dot{H}}{H^{2}}=2\left(\varepsilon_{H}-\eta_{H}\right) .
$$

Differentiating Equation (2.12) and using Equation (2.5) leads to

$$
M_{P}^{2} \ddot{H}=\dot{\phi} V^{\prime}+3 H \dot{\phi}^{2} .
$$

Inserting this into Equation (3.65) and using once more Equation (2.12) gives [11]

$$
\varepsilon_{2}=2\left(\varepsilon_{1}-\frac{V^{\prime}}{H \dot{\phi}}-3\right) .
$$

The conditions $\left|\varepsilon_{1}\right|<<1,\left|\varepsilon_{2}\right|<<1$ thus implies that to first order during slow roll, $3 H \dot{\phi} \simeq-V^{\prime}$. From Equation (2.5) it then follows that $\ddot{\phi} \approx 0$. Not surprisingly we again see that the inflaton field must have a flat potential during the slow roll era.

Using first Equations (3.66) and (3.26), and then (3.64) and (3.28) we get

$$
\varepsilon_{2}=2(2 \varepsilon-\eta), \quad \varepsilon_{2} \varepsilon_{3}=2\left(\xi+8 \varepsilon^{2}-6 \varepsilon \eta\right)
$$

Inserting the definitions (3.1), we get $[17,18,26,27]$

$$
\frac{\kappa}{2} \varepsilon_{2}=\left(\frac{V^{\prime}}{V}\right)^{2}-\frac{V^{\prime \prime}}{V}=-\left(\frac{V^{\prime}}{V}\right)^{\prime}, \quad \frac{\kappa^{2}}{2} \varepsilon_{2} \varepsilon_{3}=2\left(\frac{V^{\prime}}{V}\right)^{4}-3 \frac{V^{\prime 2} V^{\prime \prime}}{V^{3}}+\frac{V^{\prime} V^{\prime \prime \prime}}{V^{2}}
$$

These expressions are valid only in the slow roll approximation. They are different from those given by Steer and Vernizzi [28]. Using Equations (3.39) and (3.49) together with the approximation $\varepsilon_{H} \approx \varepsilon$ the Hubble flow parameters can be expressed in terms of the Hubble parameter as

$$
\frac{\kappa}{2} \varepsilon_{1}=\left(\frac{H^{\prime}}{H}\right)^{2} \quad, \quad \frac{\kappa}{4} \varepsilon_{2}=\left(\frac{H^{\prime}}{H}\right)^{2}-\frac{H^{\prime \prime}}{H} \quad, \quad \frac{\kappa^{2}}{8} \varepsilon_{2} \varepsilon_{3}=2\left(\frac{H^{\prime}}{H}\right)^{4}-3 \frac{H^{\prime 2} H^{\prime \prime}}{H^{3}}+\frac{H^{\prime} H^{\prime \prime \prime}}{H^{2}} .
$$

It was noted by Vennin [26] that these expressions are exact. They follow directly from Equations (3.51) and (3.63).

The inverse of the relationships (3.69) are

$$
\varepsilon \approx \varepsilon_{1} \quad, \quad \eta \approx 2 \varepsilon_{1}-\varepsilon_{2} / 2, \quad \xi=4 \varepsilon_{1}^{2}-3 \varepsilon_{1} \varepsilon_{2}+\varepsilon_{2} \varepsilon_{3} / 2
$$

which may be written

$$
M_{P} \frac{V^{\prime}}{V}=\sqrt{2 \varepsilon_{1}} \quad, \quad M_{P}^{2} \frac{V^{\prime \prime}}{V}=2 \varepsilon_{1}-\frac{\varepsilon_{2}}{2} \quad, \quad M_{P}^{3} \frac{V^{\prime \prime \prime}}{V}=\frac{4 \varepsilon_{1}^{2}-3 \varepsilon_{1} \varepsilon_{2}+\varepsilon_{2} \varepsilon_{3} / 2}{\sqrt{2 \varepsilon_{1}}} .
$$

The corresponding formulae for the Hubble slow roll parameters are

$$
\varepsilon_{H}=\varepsilon_{1} \quad, \quad \eta_{H}=\varepsilon_{1}-\frac{\varepsilon_{2}}{2} \quad, \quad \xi_{H}=\varepsilon_{1}^{2}-\frac{3}{2} \varepsilon_{1} \varepsilon_{2}+\frac{1}{2} \varepsilon_{2} \varepsilon_{3} .
$$


Inserting the expressions (3.73) into Equations (3.20) and (3.22) we obtain the relationships

$$
\varepsilon=\varepsilon_{1}\left[1-\frac{\varepsilon_{2}}{2\left(3-\varepsilon_{1}\right)}\right]^{2}, \quad \eta=\frac{24 \varepsilon_{1}-6 \varepsilon_{2}-8 \varepsilon_{1}^{2}+10 \varepsilon_{1} \varepsilon_{2}-\varepsilon_{2}^{2}-2 \varepsilon_{2} \varepsilon_{3}}{4\left(3-\varepsilon_{1}\right)} .
$$

Using Equations (3.38), (3.64), (3.42) and (3.72) the ratio of 3 times the kinetic energy and the total energy, and the rate of change of this ratio, and of two times the kinetic energy, can be rewritten in terms of the horizon-flow parameters as follows

$$
\varepsilon_{1}=\frac{3 \dot{\phi}^{2}}{\dot{\phi}^{2}+2 V}, \dot{\varepsilon}_{1}=H \varepsilon_{1} \varepsilon_{2},\left(\dot{\phi}^{2}\right)=-\left(2 \varepsilon_{1}-\varepsilon_{2}\right) H \dot{\phi}^{2} .
$$

Schwarz et al. [11] have constructed a classification of inflation models based upon these equations. It should be noted that the validity of their classification is restricted to first order in the slow roll parameters, but does not work in general. They write:

- $\varepsilon_{2}=2 \varepsilon_{1}$ : Kinetic energy is constant. For slow roll models this is realized to 1 , order in the slow roll parameters for chaotic inflation with a quadratic potential, $V(\phi) \propto \phi^{2}$.

It was noted by Schwarz et al. [11] that a model with a constant ratio of kinetic and total energy density has $\varepsilon_{2}=0$. Equation (3.70) then gives

$$
V V^{\prime \prime}-V^{\prime 2}=0
$$

with general solution

$$
V=V_{0} e^{A \phi},
$$

where $V_{0}$ and $A$ are integration constants. Inflation with such an exponential potential will be considered later.

V. Vennin [26] has calculated the second order corrections to the first order horizon flow parameter, and found

$$
\varepsilon_{S 1}=\varepsilon_{F 1}\left(1-\frac{\varepsilon_{F 2}}{3}\right) \quad, \quad \varepsilon_{S 2}=\varepsilon_{F 2}\left(1-\frac{\varepsilon_{F 2}}{6}-\frac{\varepsilon_{F 3}}{3}\right),
$$

where $\varepsilon_{F}$ are first order parameters.

The parameters $\varepsilon_{n}$ have been used by Myrzakulov et al. [29] in order to reconstruct viable inflationary models starting from the measured values of $n_{S}$ and $r$. Their point of departure comes from an article by Mukhanov [30]. Let us first follow Mukhanov's deduction. From Equations (2.1) and (2.2) we get

$$
H^{2}=\frac{2 \kappa V}{3(1-w)}
$$

Mukhanov makes the slow roll assumption that $\dot{\phi}^{2}<<V$. It then follows from Equation (2.8) that $w \approx-1$. Hence $1-w \approx 2$, and Equation (3.80) can be approximated by Equation (2.15). Similarly when $w \approx-1$ Equation (2.9) reduces to

$$
\dot{\phi}^{2}=(1+w) V
$$

With $\varepsilon \approx \varepsilon_{H}$ we have from Equation (3.36) that

$$
\varepsilon=(3 / 2)(1+w) .
$$


This is the relationship between the first slow roll parameter and the equation of state parameter for the dominating cosmic fluid during the inflationary era. Combining this with Equation (3.80) we get

$$
\varepsilon \approx 3-\frac{\kappa V}{H^{2}} .
$$

Ballesteros and Casas [31] have given a general argument which shows that a relatively large value of $r$ and $\alpha_{S}$ lead to problems for many inflation models. The argument goes as follows. The potential is normalized as

$$
\hat{V}=V / V_{*} \quad, \quad V_{*}=V\left(\phi_{*}\right),
$$

where $\phi_{*}$ is the value of the inflaton field at the horizon crossing scale $k_{*}$. Here $k_{*}$ is called the pivot scale and was chosen by the Planck project as $k_{*}=0.05 \mathrm{Mpc}^{-1}\left(1 \mathrm{Mpc}=3.26 \times 10^{6}\right.$ light years). Using Equation (3.1) the derivatives of the normalized potential is given by the slow roll parameters as

$$
\hat{V}_{\hat{\phi}}=\sqrt{2 \varepsilon}, \quad \hat{V}_{\hat{\phi} \hat{\phi}}=\eta \quad, \quad \hat{V}_{\hat{\phi} \hat{\phi} \hat{\phi}}=\xi / \sqrt{2 \varepsilon},
$$

where $\hat{\phi}=\phi / M_{P}$. Typical values for the slow roll parameters coming from the Planck 2015 results are $\varepsilon=-5 \eta=(4 / 3) \xi=6 \times 10^{-3}$ which gives $\hat{V}{ }_{\hat{\phi} \hat{\phi} \hat{\phi}}=-0.01 \hat{V}_{{ }^{\prime}}$ and $\hat{V}{ }_{\hat{\phi} \hat{\phi} \hat{\phi}}=0.36 \hat{V}_{,}{ }_{\hat{\phi}}$ with $\hat{V}_{\hat{\phi}}=0.11$. This means that $\left|\hat{V}_{, \hat{\phi}}\right|>>\left|\hat{V}_{, \hat{\phi} \hat{\phi}}\right|<<\left|\hat{V}_{, \hat{\phi} \hat{\phi} \hat{\phi}}\right|$. These inequalities require a very special shape of the inflaton potential. Ballesteros and Casas [31] have pointed out that a value of $\xi$ which has not a sufficiently small absolute value, may trigger the breakdown of slow roll, and thus of inflation, too early.

\subsection{Ultra Slow-Roll Inflation}

Some authors have investigated a situation where the early universe enters an era with constant potential, $V=V_{0}$ for a while [32-34]. This has been termed ultra slow-roll inflation.

We shall here calculate the different slow roll parameters in such an era, illustrating that they can be rather different. We have met with this case a few times above. The relationship between the scale factor and the rate of change of the inflaton field is given in Equation (2.6). Furthermore based upon the approximate Equation (2.19) the time dependency of the inflaton field for a flat potential was calculated in Equation (2.25). We shall now give a more general approach.

Integrating the exact Equation (2.14) for a constant potential gives

$$
H=\sqrt{\frac{\kappa V_{0}}{3}} \cosh \left(\sqrt{\frac{3 \kappa}{2}} \phi\right), \quad H^{\prime}=\kappa \sqrt{\frac{V_{0}}{2}} \sinh \left(\sqrt{\frac{3 \kappa}{2}} \phi\right), \quad H^{\prime \prime}=\frac{\kappa}{2} \sqrt{3 \kappa V_{0}} \cosh \left(\sqrt{\frac{3 \kappa}{2}} \phi\right)
$$

Inserting the expression for $H^{\prime}$ into Equation (2.13) gives

$$
\dot{\phi}=-\sqrt{2 V_{0}} \sinh \left(\sqrt{\frac{3 \kappa}{2}} \phi\right) .
$$

Integration leads to

$$
\tanh \left(\frac{1}{2} \sqrt{\frac{3 \kappa}{2}} \phi\right)=K_{1} e^{-\sqrt{3 \kappa V_{0}} t}
$$

where $K_{1}$ is an arbitrary constant. Combining this with the expression for $H$ in Equation (3.86) and using that

$$
\cosh x=\frac{1+\tanh ^{2}(x / 2)}{1-\tanh ^{2}(x / 2)}
$$


we obtain

$$
H(t)=\sqrt{\frac{\kappa V_{0}}{3}} \frac{e^{2 \sqrt{3 \kappa V_{0}} t}+K_{1}^{2}}{e^{2 \sqrt{3 \kappa V_{0}} t}-K_{1}^{2}} .
$$

In order to give a simple illustration of the behavior of this class of models, and of the differences of the slow roll parameters, we choose $K_{1}=1$. Then

$$
H(t)=\sqrt{\frac{\kappa V_{0}}{3}} \operatorname{coth}\left(\sqrt{3 \kappa V_{0}} t\right) \quad, \quad \dot{H}=-\frac{\kappa V_{0}}{\sinh ^{2}\left(\sqrt{3 \kappa V_{0}} t\right)} \quad, \quad \ddot{H}=2 \sqrt{3}\left(\kappa V_{0}\right)^{\frac{3}{2}} \frac{\cosh \left(\sqrt{3 \kappa V_{0}} t\right)}{\sinh ^{3}\left(\sqrt{3 \kappa V_{0}} t\right)}
$$

The scale factor is

$$
a(t)=a_{1} \sinh \left(\sqrt{3 \kappa V_{0}} t\right)
$$

where $a_{1}$ is an arbitrary constant. In this case Equation (3.88) can be written as

$$
\cosh \left(\sqrt{\frac{3 \kappa}{2}} \phi\right)=\operatorname{coth}\left(\sqrt{3 \kappa V_{0}} t\right) .
$$

The number of e-folds of the super slow-roll era is found by inserting the expression (3.92) of the Hubble parameter into Equation (3.62) and performing the integration

$$
N=\ln \frac{\sinh \left(\sqrt{3 \kappa V_{0}} t\right)}{\sinh \left(\sqrt{3 \kappa V_{0}} t_{f}\right)},
$$

where $t_{f}$ is the point of time of the end of this era.

The potential slow roll parameters, defined in Equation (3.1), the Hubble slow roll parameters, defined in Equation (3.7), and the horizontal slow roll parameter 3, defined in Equation (6.63) and calculated from Equation (3.66), are

$$
\varepsilon=\eta=0 \quad, \quad \varepsilon_{H}=\frac{3}{\cosh ^{2}\left(\frac{1}{2} \sqrt{\frac{3 \kappa}{2}} \phi\right)}, \eta_{H}=3 \quad, \quad \varepsilon_{1}=\varepsilon_{H} \quad, \quad \varepsilon_{2}=-6 \tanh ^{2}\left(\frac{1}{2} \sqrt{\frac{3 \kappa}{2}} \phi\right)
$$

Using Equation (3.12) and once more Equation (3.66) we get

$$
\varepsilon_{H}=3 \tanh ^{2}\left(\sqrt{3 \kappa V_{0}} t\right) \quad, \quad \varepsilon_{2}=-\frac{6}{\cosh ^{2}\left(\sqrt{3 \kappa V_{0}} t\right)} .
$$

Due to Equation (3.94) these expressions are equivalent to those in Equation (3.96). Hence in this case we have part of an inflationary era with large values of the slow roll parameters. These values are not related to the observable spectral parameters. They illustrate, however, how different the potential-, the Hubble-, and the horizontal slow roll parameters can be.

\section{Power Spectra of Primordial Fluctuations}

\subsection{Spectral Parameters}

We shall here review the mathematical quantities that are used to describe the temperature fluctuations in the CMB. The power spectra of scalar and tensor fluctuations are represented by [5]

$$
\begin{aligned}
& P_{S}=A_{S}\left(k_{*}\right)\left(\frac{k}{k_{*}}\right)^{n_{S}-1+(1 / 2) \alpha_{S} \ln \left(k / k_{*}\right)+\cdots}, P_{T}=A_{T}\left(k_{*}\right)\left(\frac{k}{k_{*}}\right)^{n_{T}+(1 / 2) \alpha_{T} \ln \left(k / k_{*}\right)+\cdots}, \\
& A_{S}=\frac{V}{24 \pi^{2} \varepsilon M_{P}^{4}}=\left(\frac{H^{2}}{2 \pi \dot{\phi}}\right)^{2}, \quad A_{T}=\frac{2 V}{3 \pi^{2} M_{P}^{4}}=\varepsilon\left(\frac{2 H^{2}}{\pi \dot{\phi}}\right)^{2}
\end{aligned}
$$

Here $k$ is the wave number of the perturbation that is a measure of the average spatial extension for a perturbation with a given power, and $k_{*}$ is the value of $k$ at a reference scale usually chosen as the 
scale at horizon crossing, called the pivot scale. One often writes $k=\dot{a}=a H$, where $a$ is the scale factor representing the ratio of the physical distance between reference particles in the universe relative to their present distance. The quantities $A_{S}$ and $A_{T}$ are amplitudes at the pivot scale, and $n_{S}$ and $n_{T}$ are the spectral indices of the scalar and tensor fluctuations. The quantity $\delta_{n s} \equiv 1-n_{s}$ is called the tilt of the power spectrum of curvature perturbations because it represents the deviation of the value $n_{S}=1$ which represents a scale invariant spectrum. In the present article we shall represent $n_{S}$ by $\delta_{n s}$.

Furthermore $\alpha_{S}$ and $\alpha_{T}$ are factors representing the $k$-dependence of the spectral indices. They are called the running of the spectral indices and are defined by

$$
\alpha_{S}=\frac{d n_{S}}{d \ln k}, \quad \alpha_{T}=\frac{d n_{T}}{d \ln k} .
$$

If $n_{S}=1$ the spectrum of the scalar fluctuations is said to be scale invariant. An invariant mass-density power spectrum is called a Harrison-Zel'dovich spectrum. One of the predictions of the inflationary universe models is that the cosmic mass distribution has a spectrum that is nearly scale invariant, but not exactly. The observations and analysis of the Planck team $[1,7,35,36]$ have given $n_{S}=0.968 \pm 0.006$. Hence we shall use $n_{S}=0.968$ as the preferred value of $n_{S}$. Furthermore, they have obtained $\alpha_{S}=-0.003 \pm 0.007$. Adding data from other measurements Huang [37] gives $\alpha_{S}=-0.006 \pm 0.007$. Different inflationary models will be evaluated against the Planck 2015 value of the tilt of the curvature fluctuations, $\delta_{n s}=0.032$. A combination of all the relevant experiments gives the restriction $n_{t}<0.36$.

The tensor-to-scalar ratio $r$ is defined by

$$
r \equiv \frac{P_{T}\left(k_{*}\right)}{P_{S}\left(k_{*}\right)}=\frac{A_{T}}{A_{S}}
$$

As noted by Baumann [38], the tensor-to-scalar ratio is a measure of the energy scale of inflation, $V^{1 / 4}=(100 r)^{1 / 4} 10^{16} \mathrm{GeV}$. From Equations (4.1) and (4.3) we have

$$
r=16 \varepsilon .
$$

\subsection{The BICEP2 Announcment}

The seventeenth of March 2014 the BICEP2 team announced [39] the possible discovery of B-mode signals in the cosmic microwave radiation corresponding to a tensor-to-scalar ratio $r=0.20$. They estimated that $20 \%$ of the signal came from dust in the Milky way, and hence that there was a contribution of magnitude $r=0.16$ from primordial gravitational waves that were produced in the inflationary era.

This inspired researchers working in this field to produce a great number of papers on this topic, several hundred in one year. However one year later, after having made a thorough analysis of the observational data together, the Planck and BICEP2 teams published a report together [2] concluding that all of the detected signal might be due to galactic dust. But the uncertainty is still so large that a signal representing a value of $r$ of the order of a few percent may be hidden behind the galactic curtain of dust.

Some of the results that were produced partly as a result of the original BICEP2 announcement will here be reviewed. The first observational result that was discussed was the discrepancy between the Planck result that had been established prior to the BICEP2 announcement, that $r<0.12$ and the BICEP2 result. This was overcome in several ways.

The researchers immediately noted that the observations of Planck and BICEP were performed at different scales. Hence the problem of reconciling the results could be solved by a sufficiently large scale dependence of the value of $r$. For example Ashoorioon and coworkers [40] noted that agreement could be obtained if $\alpha_{T} \geq 0.16$. They constructed an inflationary scenario in agreement 
with the BICEP2-Planck 2014 resultsthat were based upon a non Bunch-Davis initial state for cosmic perturbations. Due to the BICEP2-Planck 2015 result we will not consider this here.

The tensor-to-scalar ratio can be determined from observations of the B-mode of the polarization of the CMB. In the measured wavelength region this B-mode pattern is partly due to radiation from galactic dust and partly to imprints on the CMB at the time 380,000 years after the Big Bang, when the universe became transparent for the $\mathrm{CMB}$, from relic gravitational waves that were produced by quantum fluctuations in the inflationary era.

As mentioned above the BICEP2 team recently announced $[1,40]$ that they have measured the B-mode in the CMB-polarization. Disregarding a possible contribution from the foreground they obtained a best fit value $r=0.20$. Subtracting a contribution $r_{f}=0.04$ due to the foreground according to a preferred model, they arrived at $r=0.16$. In September 2014 the experimental bounds on $n_{S}$, $r$ and $\alpha_{S}$ were summarized as follows [41-43]: $n_{S}=0.957 \pm 0.015, r=0.16_{-0.05}^{+0.06}, \alpha_{S}=-0.022_{-0.021}^{+0.020}$. In October 2016 Benetti and Ramos [44] gave $\alpha_{s}=0.011 \pm 0.014$ while Ballesteros and Casas [31] gave a smaller uncertainty, $\alpha_{S}=-0.018 \pm 0.009$, excluding values very close to zero. In January 2016 Bamba et al. [45] gave, $n_{S}=0.968 \pm 0.006, r<0.11, \alpha_{S}=-0.003 \pm 0.007$. When considering the consequences for the inflationary models of the observations we shall here mostly use the center values given in [35,36], namely $n_{S}=0.968$, i.e., $\delta_{n s}=0.032, \alpha_{S}=-0.003$ and $r=0.05$. With $r=0.05$ Equation (4.4) gives $\varepsilon=0.003$. These will be called the BPK-values (BICEP2, Planck, Keck). The most recent analysis [46] of the BKP-data concludes that $r<0.04$, which will also be used in the confrontation of different inflationary models with observational data.

It follows from Equations (3.82) and (4.4) that the equation of state parameter during the slow roll era is given in terms of the tensor-to-scalar ratio as

$$
1+w=r / 24
$$

With $r=0.05$ this gives $1+w=0.002$ during the slow roll era. According to Equation (2.21) this corresponds to power law expansion with an extremely large exponent, $a=a_{1}\left(t / t_{1}\right)^{16 / r}=a_{1}\left(t / t_{1}\right)^{320}$ during the slow roll era.

\subsection{The Lyth Bound}

Assuming that $\varepsilon_{\min }$ in Equation (3.61) is equal to the value of $\varepsilon$ at the slow roll period we can use Equation (4.4) to express the Lyth bound in terms of the scalar to-tensor ratio [23],

$$
r<2\left(\frac{2 \Delta \phi}{N M_{P}}\right)^{2}
$$

This form of the Lyth bound tells that in general $r$ will have very small values for small field inflation with $\Delta \phi<M_{P}$. Lyth [21] and Minor and Kaplinghat [47] have argued that the right hand side of Equation (4.6) gives an estimate of the order of magnitude of $r$ predicted by different classes of inflationary models.

The Lyth bound can also be written [48,49]

$$
\Delta \phi>N \sqrt{r / 8} M_{P},
$$

where $\Delta \phi$ is the change of the value of the scalar field during the slow roll era. Hence small field inflation requires $N \sqrt{r / 8}<1$ or $r<8 / N^{2}$. In order to solve the horizon problem the number of e-fold must be at least $N \simeq 50$. Then small field inflation demands $r<0.003$ [50]. 


\subsection{Relationships between the Spectral Indices and the Slow Roll Parameters}

We shall now find how the spectral indices depend upon the slow roll parameters. From Equation (4.1) it follows that they are given by

$$
\delta_{n s}=-\left[\frac{d \ln P_{S}(k)}{d \ln k}\right]_{k=a H^{\prime}} \quad n_{T}=\left[\frac{d \ln P_{T}(k)}{d \ln k}\right]_{k=a H} .
$$

The quantities inside the brackets are evaluated at the horizon crossing where $k=k_{*}$, and the wave number is equal to the scale factor times the Hubble parameter. It will be useful to write

$$
\frac{d}{d \ln k}=\frac{d}{d N} \times \frac{d N}{d \ln k}
$$

Hence, using that $A_{S} \propto H^{2} / \varepsilon$, the scalar spectral indices may be written as

$$
\delta_{n s}=\left(\frac{d \ln \varepsilon}{d N}-2 \frac{d \ln H}{d N}\right) \frac{d N}{d \ln k} \quad, \quad n_{T}=2 \frac{d \ln H}{d N} \frac{d N}{d \ln k} .
$$

Using Equations (3.48) and (3.12), we get in the slow roll approximation

$$
\frac{d \ln H}{d N}=-\frac{\dot{H}}{H^{2}}=\varepsilon_{H}
$$

From the condition that the spectral indices are calculated at the horizon crossing we have $k=a H$. Equation (3.46) gives $d N=-d \ln a$. Hence $d \ln k=d \ln a+d \ln H=-d N+d \ln H$. Since $H$ is approximately constant during the slow roll inflationary era, it follows that

$$
\frac{d}{d \ln k} \approx-\frac{d}{d N}
$$

Inserting this together with Equations (3.58) and (4.11) into Equation (4.10) leads to

$$
\delta_{n s}=2(3 \varepsilon-\eta)
$$

Using Equation (3.5) this equation can be written as

$$
\delta_{n s}=2\left[\varepsilon-(\sqrt{2 \varepsilon / \kappa})^{\prime}\right] .
$$

Equations (3.26) and (4.10)-(4.13) give

$$
n_{T} \approx-2 \varepsilon
$$

A consistency relation between $r$ and $n_{T}$ follows from Equations (4.4) and (4.15)

$$
n_{T}=-\frac{r}{8}
$$

This relationship is model independent, and must be taken into account by inflationary models in general. According to this relationship the BICEP2/Planck preferred value $0<r<0.05$ gives $-0.006<n_{T}<0$. This is not in agreement with the combined BICEP2/Planck and LIGO data which give $n_{T}=-0.76_{-0.52}^{+1.37}$ [51]. However the BICEP/Planck data alone constrain the tensor tilt to $n_{T}=0.66_{-1.44}^{+1.83}$, so the actual value of $n_{T}$ is presently quite uncertain. However if it turns out that future observations imply a large absolute value of $n_{T}$, say $\left|n_{T}\right|>2$, then most present inflationary models are in trouble. While inflationary models obeying the consistency relationship (4.16) predict a small absolute value of $n_{T}$, the ekpyrotic universe model with colliding branes has an opposite 
problem. Huang and Wang [51] noted that such models predicts $n_{T}=2$, and that observational data, including the LIGO data, rule out the ekpyrotic universe model.

Martin [19] has noted that in general we have six independent spectral quantities, $n_{S}$ (or $\delta_{n s}$ ), $n_{T}, \alpha_{S}, \alpha_{T}, \beta_{S}, \beta_{T}$, where

$$
\beta_{S}=\frac{d \alpha_{S}}{d \ln k} \quad, \quad \beta_{T}=\frac{d \alpha_{T}}{d \ln k} .
$$

The quantities $\beta$ are called the running of the running. Martin further pointed out that the predictions of slow roll inflation can be expressed in terms of 3 slow roll parameters. Hence, there exist three consistency relations between the spectral parameters. The three parameters describing the tensor sector can be expressed in terms of those describing the scalar sector.

In Section 5.1 we shall need the generalizations of Equations (4.4), (4.14), and (4.15) that are accurate to second order in the slow roll parameters [52],

$$
\begin{gathered}
\delta_{n s}=2(3 \varepsilon-\eta)+2 \varepsilon(7 \varepsilon-3 \eta)+8 C \varepsilon(3 \varepsilon-2 \eta)+2 C \xi, \\
r=16 \varepsilon[1+2 C(2 \varepsilon-\eta)], \quad n_{T}=-2 \varepsilon[1+\varepsilon+2(1+C)(2 \varepsilon-\eta)],
\end{gathered}
$$

where $C=-0.73$. Using the approximate version $\varepsilon \approx \varepsilon_{H}$ of Equation (3.26) $\delta_{n s}, n_{T}$ and $r$ can to lowest order be expressed in terms of the Hubble slow roll parameters as ([14] with $\sigma \rightarrow-\delta_{n s}$ )

$$
\delta_{n s}=2\left(2 \varepsilon_{H}-\eta_{H}\right) \quad, \quad n_{T}=-2 \varepsilon_{H} \quad, \quad r=16 \varepsilon_{H} .
$$

Inserting the expressions (3.20) and (3.22) into Equations (4.18) and (4.19), we have to second order [53]

$$
\delta_{n s}=2\left[2 \varepsilon_{H}-\eta_{H}+C \xi+4(1+C) \varepsilon_{H}^{2}+(3+5 C) \varepsilon_{H} \eta_{H}\right] \quad, \quad r=16 \varepsilon_{H}\left[1+2 C\left(\varepsilon_{H}-\eta_{H}\right)\right] .
$$

From Equations (4.14), (3.27), and (3.22) we obtain

$$
\delta_{n s, N}=2\left(12 \varepsilon^{2}-\xi-4 \varepsilon \delta_{n s}\right) .
$$

Combining this with Equation (3.24) we get [14]

$$
\delta_{n s, N}=12 \varepsilon_{H}^{2}-2 \xi_{H}-5 \varepsilon_{H} \delta_{n s} .
$$

From Equations (4.4) and (4.14) we have

$$
\varepsilon=\frac{r}{16} \quad, \quad \eta=\frac{1}{16}\left(3 r-8 \delta_{n s}\right) .
$$

Inserting the Planck and BICEP2 values $\delta_{n s}=0.032$ and $r=0.05$ gives $\varepsilon=0.003, \eta=-0.007$. With $r<0.04$ we have $\varepsilon<0.0025$. For $r=(8 / 3) \delta_{n s}$ we have $\eta \approx 0$ which happens if the inflaton potential is $V(\phi)=A \phi+B$. It may be noted that $r=0$ gives $\eta=-0.016$. The corresponding formulae for the Hubble slow roll parameters are

$$
\varepsilon_{H}=\frac{r}{16} \quad, \quad \eta_{H}=\frac{1}{8}\left(r-4 \delta_{n s}\right) .
$$

Equation (4.13) implies that an inflationary universe model with a scale invariant spectrum has $\eta=3 \varepsilon$ or equivalently $\eta_{H}=2 \varepsilon_{H}$ [54]. Inserting the expressions (3.1) we get the differential equation

$$
2 V V^{\prime \prime}-3 V^{\prime 2}=0
$$


with general solution

$$
V(\phi)=(A \phi+B)^{-2},
$$

where $A$ and $B$ are arbitrary constants [55].

The running of the spectral index of scalar fluctuations may also be expressed in terms of the slow roll parameters. From Equations (4.2), (4.12), and (3.51), the first of Equations (3.1), and (3.11) it follows that

$$
\alpha_{S}=-\frac{1}{\kappa} \frac{V^{\prime}}{V} \frac{d n_{S}}{d \phi}=-\sqrt{\frac{2 \varepsilon}{\kappa}} n_{s}^{\prime}=\frac{1}{H} \dot{n}_{S}=-\frac{1}{H} \dot{\delta}_{n s}
$$

with—for $V^{\prime}>0$ and + for $V^{\prime}<0$. Using this together with Equations (4.22) and (3.48) and then (3.22), (3.24) we obtain,

$$
\alpha_{S}=8 \varepsilon(2 \eta-3 \varepsilon)-2 \xi=2 \varepsilon_{H}\left(5 \eta_{H}-4 \eta_{H}\right)-2 \xi_{H} .
$$

From Equations (3.5), (3.6) and (4.24) we get

$$
\varepsilon^{\prime \prime}=\xi-\frac{1}{8}\left(\frac{9}{32} r^{2}-r \delta_{n s}-2 \delta_{n s}^{2}\right) .
$$

With more accurate observations than we have presently it may also be possible to falsify inflationary models by considering the running of the running of the scalar spectral index, $\beta_{S}$. This quantity is given in terms of the slow roll parameters and the fourth derivative of the inflaton potential by $[27,56]$,

$$
\beta_{S}=-32 \varepsilon\left(6 \varepsilon^{2}-6 \varepsilon \eta+\eta^{2}\right)-2 \xi(12 \varepsilon-\eta)+2 \sigma,
$$

or by using Equations (3.5) and (3.11),

$$
\beta_{S}=-32 \varepsilon\left(6 \varepsilon^{2}-6 \varepsilon \eta+\eta^{2}\right)-16 \varepsilon \xi-2 \dot{\xi} / H .
$$

Huang [37] have pointed out that observational data already lead some restrictions on $\beta_{S}$. From the Planck-data he found $\beta_{S} \simeq 0.025 \pm 0.013$. This is in good agreement with the analysis of Benetti and Ramos [44], giving $\beta_{s} \simeq 0.029 \pm 0.015$. Inserting (4.4) and (4.13) into Equation (4.29) gives $\xi$ in terms of observable quantities,

$$
\xi=\frac{r}{64}\left(3 r-16 \delta_{n s}\right)-\frac{\alpha_{S}}{2} .
$$

The Planck/BICEP2 data are $\delta_{n s}=0.032 \pm 0.005, r<0.04, \alpha_{S}=-0.003 \pm 0.007$. The value of $r$ giving the smallest value of $\xi$ is

$$
r_{1}=(8 / 3) \delta_{n s}=0.085 \pm 0.013
$$

The corresponding minimum value of $\xi$ is

$$
\xi_{\min }=-(1 / 2) \alpha_{S}-(1 / 3) \delta_{n s}^{2} .
$$

Inserting the Planck $/$ BICEP2 data gives $-0.002<\xi_{\min }<0.005$. Thus, inflationary models that predict $\xi<<-0.002$ are disfavored by the Planck/BICEP2 data.

From Equations (4.30) and (4.33) we have

$$
\varepsilon^{\prime \prime}=\frac{1}{256}\left(r-8 \delta_{n s}\right)\left(3 r-8 \delta_{n s}\right)-\frac{\alpha_{s}}{2} .
$$


Using Equations (4.22) and (4.31) the running of the running of the spectral index $n_{S}$ can be written

$$
\beta_{S}=\frac{1}{2} \alpha_{S} \delta_{n S}+\frac{9}{16} \alpha_{S} r-\frac{r}{4}\left(\delta_{n S}^{2}-\frac{15}{16} \delta_{n s} r+\frac{15}{128} r^{2}\right)+2 \sigma .
$$

It may also be noted that during the slow roll inflationary era the running of the spectral index of scalar fluctuations may be written

$$
\alpha_{S} \approx-n_{S, N}=\delta_{n S, N}
$$

From Equations (3.25) and (4.30) we have

$$
\xi_{H}=(1 / 128)\left(3 r^{2}-20 r \delta_{n s}-64 \alpha_{S}\right) .
$$

The rate of change of the slow roll parameters can now be expressed in terms of observable quantities. From Equations (3.5) and (4.24) we obtain

$$
\sqrt{\frac{2 \varepsilon}{\kappa}} \varepsilon^{\prime}=\frac{1}{128} r\left(r-8 \delta_{n S}\right), \quad \sqrt{\frac{2 \varepsilon}{\kappa}} \eta^{\prime}=\frac{1}{128}\left(3 r^{2}-24 r \delta_{n S}-64 \alpha_{S}\right) .
$$

Correspondingly we find using Equations (3.26) and (4.40) for the rate of change of the Hubble slow roll parameters,

$$
\sqrt{\frac{2 \varepsilon}{\kappa}} \varepsilon_{H}^{\prime}=\frac{1}{128} r\left(r-8 \delta_{n S}\right) \quad, \quad \sqrt{\frac{2 \varepsilon}{\kappa}} \eta_{H}^{\prime}=\frac{1}{64}\left(r^{2}-8 r \delta_{n s}-32 \alpha_{S}\right) .
$$

The running of the spectral index of tensor fluctuations is

$$
\alpha_{T}=-\sqrt{\frac{2 \varepsilon}{\kappa}}\left(n_{T}\right)^{\prime}=2 \sqrt{\frac{2 \varepsilon}{\kappa}} \varepsilon^{\prime} .
$$

Inserting the expression for $\varepsilon^{\prime}$ from Equation (3.5) we get [57]

$$
\alpha_{T}=4 \varepsilon(\eta-2 \varepsilon) \text {. }
$$

Applying the approximation $\varepsilon \approx \varepsilon_{H}$ and using Equations (3.20), (3.22), and (3.72), we get to second order in the slow roll parameters

$$
\alpha_{T} \simeq 4 \varepsilon_{H}\left(\eta_{H}-\varepsilon_{H}\right) \simeq 4 \varepsilon_{1}\left(\varepsilon_{1}-\frac{3}{2} \varepsilon_{2}\right) .
$$

A constant spectral index of the tensor fluctuations require $\eta=2 \varepsilon$ which corresponds to

$$
V V^{\prime \prime}-V^{\prime 2}=0
$$

The general solution of this equation is

$$
V(\phi)=V_{0} e^{A \phi},
$$

where $A$ is an arbitrary constant and $V_{0}=V(0)$.

We also have

$$
\alpha_{T} \approx-n_{T, N}
$$


A second consistency relation, this time between $\alpha_{T}, r$ and $\delta_{n s}$ follows from Equations (4.20) and (4.43) as was shown by Carrillo-González et al. [58],

$$
\alpha_{T}=-\frac{r}{64}\left(8 \delta_{n s}-r\right) .
$$

Inserting the Planck-BICEP2 values $\delta_{n s}=0.032,0<r<0.04$ gives $-0.00014<\alpha_{T}<0$. Due to the great observational uncertainty in the value of $n_{T}$, it is at the present time not possible to give a restriction on the value of $\alpha_{T}$ directly from observations.

Ballesteros and Casas [31] have defined a running of the tensor-to-scalar ratio,

$$
\alpha_{r} \equiv \frac{d r}{d \ln k} .
$$

It follows from Equations (4.42) and (4.49) that

$$
\alpha_{r}=\left(\delta_{n s}-\frac{r}{8}\right) r
$$

A related quantity was considered by Ashoorioon and coworkers [40],

$$
\hat{\alpha}_{r} \equiv \frac{d \ln r}{d \ln k} .
$$

It follows from Equation (4.3) that

$$
\hat{\alpha}_{r}=\frac{d \ln P_{T}}{d \ln k}-\frac{d \ln P_{S}}{d \ln k} .
$$

From Equation (4.8) it now follows that

$$
\hat{\alpha}_{r}=n_{T}+\delta_{n s} .
$$

Using Equation (4.17) we have

$$
\hat{\alpha}_{r}=\delta_{n s}-\frac{r}{8} .
$$

It may be noted that models with $r=8 \delta_{n s}$ has no running of the tensor-to-scalar ratio or the tilt of the tensor fluctuations. These models are ruled out by the BPK-observations.

It follows from Equation (4.16) and the BICEP2 result that the tilt of the power spectrum of the tensor modes of the CMB-spectrum should have a negative value, $n_{T}=-0.005$. This has been further discussed by Chen and Huang $[59,60]$.

It follows from Equation (4.47) that the expressions in Equation (4.40) may be written

$$
\sqrt{\frac{2 \varepsilon_{H}}{\kappa}} \varepsilon_{H}^{\prime}(\phi)=\frac{\alpha_{T}}{2}, \quad \sqrt{\frac{2 \varepsilon_{H}}{\kappa}} \eta_{H}^{\prime}(\phi)=\alpha_{T}-\frac{\alpha_{S}}{2} .
$$

From Equations (4.13), (4.4), (4.15), (4.17) and (4.42) and using Equation (3.72) we have to first order in the slow roll parameters

$$
\delta_{n s}=2 \varepsilon_{1}+\varepsilon_{2} \quad, \quad n_{T}=-2 \varepsilon_{1} \quad, \quad r=16 \varepsilon_{1} \quad, \quad \alpha_{S}=-2 \varepsilon_{1} \varepsilon_{2}-\varepsilon_{2} \varepsilon_{3} \quad, \quad \alpha_{T}=-2 \varepsilon_{1} \varepsilon_{2} .
$$

The inverse equations are

$$
\varepsilon_{1}=\frac{r}{16} \quad, \quad \varepsilon_{2}=\delta_{n s}-\frac{r}{8}=-8 \frac{\alpha_{T}}{r} \quad, \quad \varepsilon_{2} \varepsilon_{3}=\alpha_{T}-\alpha_{S} .
$$


Furthermore, the running of the running for the tensor mode is [19]

$$
\beta_{T}=-2 \varepsilon_{1} \varepsilon_{2}\left(\varepsilon_{2}+\varepsilon_{3}\right)
$$

Inserting the expressions (4.57) into Equation (4.58) gives the third consistency relation

$$
\beta_{T}=\frac{r}{8}\left[\alpha_{S}-\left(\delta_{n s}-\frac{r}{4}\right)\left(\delta_{n s}-\frac{r}{8}\right)\right] .
$$

It may be noted that the BKP-values give the very small value $\beta_{T}=2 \times 10^{-5}$. A confrontation against observations is presently not possible.

As pointed out by Martin [19], inserting the BICEP2/Planck data gives the following constraints, $\varepsilon_{1}<0.007$ and $\varepsilon_{2}=0.030_{-0.006}^{+0.007}$, and almost no constraints on $\varepsilon_{3}$. This has consequences for the shape of the inflaton potential. From $\varepsilon_{1} \approx \varepsilon$ and Equation (3.70) we get

$$
V^{\prime}(\phi)=\sqrt{2 \varepsilon_{1}}\left(V / M_{P}\right) \quad, \quad V^{\prime \prime}(\phi)=\left(2 \varepsilon_{1}-\varepsilon_{2} / 2\right)\left(V / M_{P}^{2}\right) .
$$

The BICEP2/Planck constraints on $\varepsilon_{1}$ and $\varepsilon_{2}$ then lead to $\left|v^{\prime}(\phi)\right|<0.14\left(V / M_{P}\right)$ and $-0.03\left(V / M_{P}^{2}\right)<V^{\prime \prime}(\phi)<0.008\left(V / M_{P}^{2}\right)$.

To second order in the slow roll parameters the expressions of the spectral parameters in terms of the horizon-flow parameters are found by inserting the transformations in Equation (3.72) into Equations (4.18) and (4.19). This gives

$$
\begin{aligned}
& \delta_{n s}=2 \varepsilon_{1}+\varepsilon_{2}+2 \varepsilon_{1}^{2}+(3+2 C) \varepsilon_{1} \varepsilon_{2}+C \varepsilon_{2} \varepsilon_{3} \\
& r=16 \varepsilon_{1}\left(1+C \varepsilon_{2}\right), \quad n_{T}=-2 \varepsilon_{1}\left[1+\varepsilon_{1}+(1+C) \varepsilon_{2}\right] . \\
& \alpha_{s}=-\varepsilon_{2}\left(2 \varepsilon_{1}+\varepsilon_{3}\right), \quad \alpha_{T}=-2 \varepsilon_{1} \varepsilon_{2}
\end{aligned}
$$

The first two expressions are slightly different from those of Barbosa-Candejas et al. [61].

\subsection{Inflection-Point Inflation}

As an illustration of the application of the formalism we shall here consider inflection-point inflation, which is a model of inflation near an inflection point. Inflation near inflection points have been investigated by several researchers [62-64].

Close to the inflection point with $\phi=\phi_{0}$ the inflaton potential can be written

$$
V=V_{0}+\lambda_{1}\left(\phi-\phi_{0}\right)+\frac{1}{2} \lambda_{2}\left(\phi-\phi_{0}\right)^{2}+\frac{1}{6} \lambda_{1}\left(\phi-\phi_{0}\right)^{3},
$$

where $V_{0}=V\left(\phi_{0}\right)$. Then the potential slow roll parameters are

$$
\begin{aligned}
& \varepsilon=\frac{M_{P}^{2}}{2}\left(\frac{\lambda_{1}+\lambda_{2}\left(\phi-\phi_{0}\right)+\left(\lambda_{3} / 2\right)\left(\phi-\phi_{0}\right)^{2}}{V_{0}+\lambda_{1}\left(\phi-\phi_{0}\right)+\frac{1}{2} \lambda_{2}\left(\phi-\phi_{0}\right)^{2}+\frac{1}{6} \lambda_{1}\left(\phi-\phi_{0}\right)^{3}}\right)^{2}, \\
& \eta=M_{P}^{2} \frac{\lambda_{2}+\lambda_{2}\left(\phi-\phi_{0}\right)}{V_{0}+\lambda_{1}\left(\phi-\phi_{0}\right)+\frac{1}{2} \lambda_{2}\left(\phi-\phi_{0}\right)^{2}+\frac{1}{6} \lambda_{1}\left(\phi-\phi_{0}\right)^{3}} .
\end{aligned}
$$

We shall first follow Okada et al. [64] and evaluate the tensor-to-scalar ratio at the inflection point. At this point the slow roll parameters reduce to

$$
\varepsilon\left(\phi_{0}\right)=\frac{M_{P}^{2}}{2}\left(\frac{\lambda_{1}}{V_{0}}\right)^{2}, \quad \eta\left(\phi_{0}\right)=M_{P}^{2} \frac{\lambda_{2}}{V_{0}} .
$$


They then used the Planck data to obtain

$$
\frac{\lambda_{1}}{\phi_{0}^{3}} \approx 2 \times 10^{3}\left(\frac{\phi_{0}}{M_{P}}\right)^{3}\left(\frac{V_{0}}{\phi_{0}^{4}}\right)^{3 / 2}, \quad \frac{\lambda_{2}}{\phi_{0}^{2}} \approx-0.5 \delta_{n s}\left(\frac{\phi_{0}}{M_{P}}\right)^{2} \frac{V_{0}}{\phi_{0}^{4}} .
$$

Hence

$$
\varepsilon\left(\phi_{0}\right) \approx 2 \times 10^{6} \frac{V_{0}}{M_{P}^{4}} \quad, \quad \eta\left(\phi_{0}\right) \approx-0.5 \delta_{n s} .
$$

From (4.4) and (4.13) we have

$$
r=16 \varepsilon=\frac{8}{3}\left(\delta_{n s}+2 \eta\right)
$$

This leads to an inconsistency. Together with the last of the Equations (4.66) it gives $r=0$, which together with (4.64) and (4.66) requires $\lambda_{1}=V_{0}=0$. Hence with these values it does not work to evaluate the spectral parameters at the inflection point.

We will now briefly review the more general treatment of inflection point inflation given by Choi and Lee [63]. They calculated the spectral parameters from the expressions (4.63) and found the $r, \delta_{n s}-$ relation

$$
r=\frac{1}{3}\left[8 \delta_{n s}+4 \sqrt{\frac{r \lambda_{3}}{\lambda_{1}}} \sin (2 \theta)\right],
$$

where the value of $\sin (2 \theta)$ must either be assumed or determined from measured values of the spectral parameters, and

$$
\frac{\lambda_{1}}{\lambda_{3}}=91\left(\frac{N_{\max }}{120}\right)^{2} r \cos ^{4} \theta
$$

For $\theta=0$ the tensor-to-scalar ratio is

$$
r_{0}=\frac{8}{3} \delta_{n s}
$$

With the Planck value $\delta_{n s}=0.032$ we have $r_{0}=0.085$. Inserting Equation (4.69) with $N_{\max }=115$ into Equation (4.68) gives

$$
\tan \theta=3\left(r-r_{0}\right) .
$$

Recent analysis of the BICEP2, Planck and Keck data [46] indicate that $r<0.04$ and hence that $r<r_{0}$. Then $\tan \theta<0$. According to Choi and Lee

$$
\tan \theta=\sqrt{\frac{\lambda_{3}}{2 \lambda_{1}}}\left(\phi_{*}-\phi_{0}\right)
$$

Hence, this requires that $\phi_{*}<\phi_{0}$.

Choi and Lee have further shown that

$$
V_{0}=3.25 \times 10^{-8} r \quad, \quad \lambda_{1}=1.26 \times 10^{-10}\left(\frac{120}{N_{\max }}\right)^{2} \sqrt{r} \sec ^{2} \theta \quad, \quad \lambda_{2}=1.15 \times 10^{-8} r^{3 / 2} \cos ^{2} \theta .
$$

Inserting Equation (4.71) and using the value $N_{\max }=115$ gives

$$
\lambda_{1}=\frac{1.15 \times 10^{-8} r^{3 / 2}}{1+9\left(r-r_{0}\right)^{2}} \quad, \quad \lambda_{3}=1.37 \times 10^{-10} \sqrt{r}\left[1+9\left(r-r_{0}\right)^{2}\right] .
$$

With $r<0.04$ we get $V_{0}<1.3 \times 10^{-9}, \lambda_{1}<9.2 \times 10^{-11}, \lambda_{3}>>2.7 \times 10^{-11}$. 


\section{The N-Formalism}

K. Bamba, S. Nojiri and S. D. Odintsov [65], and Garcia-Bellido and Roest [66,67] have independently of each other introduced a new so-called $N$-formalism, which is useful in calculating the physical parameters characterizing the observable properties of the CMB-radiation. It has been further developed by Bamba and Odintsov [68]. In this formalism the spectral quantities are expressed by the slow roll parameter $\varepsilon$ and its derivatives with respect to the number of e-folds, $N$. From the first of the Equation (3.58), together with the Equations (4.4), (4.13), and (4.15), we have

$$
\delta_{n s}=2 \varepsilon-(\ln \varepsilon)_{N} \quad, \quad r=16 \varepsilon, \quad n_{T}=-2 \varepsilon,
$$

where ${ }_{N}$ means derivative with respect to $N$. It may be noted from Equation (4.40) that if $\varepsilon=$ constant then

$$
r=8 \delta_{n s}
$$

which is larger than allowed by the Planck data.

Using Equations (4.28), (3.50) and (4.42) give for the running of the spectral indices

$$
\alpha_{S}=\delta_{n s, N} \quad, \quad \alpha_{T}=2 \varepsilon, N
$$

These expressions are different from those in Equation (II.13) of Bamba et al. [68]. It follows from Equation (3.55), which is valid in the slow roll approximation, that when $\varepsilon$ is given as a function of $N$ the potential $V$ is found as a function of $N$ from

$$
V(N)=\exp \left[2 \int \varepsilon(N) d N\right]
$$

It may be noted that the inverse procedure of Chiba [69] for calculating the potential from the spectral index $n_{S}$ by means of the formulae (5.1) and (5.4) is not mathematically equivalent to the calculation of $n_{S}$ from the potential. These procedures give the same result only in the large $N$ limit.

Barbosa-Cendejas et al. [61] have expressed this formalism in terms of the parameter $\varepsilon_{1}=\varepsilon_{H}$. From Equation (3.53) we have

$$
H(N)=H_{0} \exp \left[\int_{N}^{0} \varepsilon_{1}(N) d N\right] .
$$

Equations (3.65) and (3.70) give

$$
\varepsilon_{2}=-\frac{\varepsilon_{1, N}}{\varepsilon_{1}} \quad, \quad \varepsilon_{2} \varepsilon_{3}=\frac{\varepsilon_{1, N N}}{\varepsilon_{1}}-\left(\frac{\varepsilon_{1, N}}{\varepsilon_{1}}\right)^{2} .
$$

From these equations and Equation (4.61) it follows that all the cosmological observables can be expressed by the $\varepsilon_{1}(N)$ parameter.

The inflaton field as a function of $N$ can be found by writing Equation (3.50) as

$$
\phi(N)=\sqrt{\frac{2}{\kappa}} \int \sqrt{\varepsilon_{1}(N)} d N .
$$

Using the $N$-formalism Roest [70], Mukhanov [71], and Garcia-Bellido et al. [66,67] have recently classified a large number of inflationary universe models into so-called universality classes. In these classes the slow roll parameters $\varepsilon, \eta$ and $\xi$ have an asymptotic power law dependence on the number $N$ of e-folds. They consider several inflationary models of this type. 


\subsection{Constant \& Class}

This class of inflationary models has constant value of the slow roll parameter $\varepsilon=\varepsilon_{0}$. Then the spectral parameters as calculated from Equations (4.14), (4.28) and (4.42) are

$$
\delta_{n s}=2 \varepsilon_{0} \quad, \quad r=16 \varepsilon_{0} \quad, \quad n_{T}=-2 \varepsilon_{0} \quad, \quad \alpha_{S}=\alpha_{T}=0
$$

The Planck value $\delta_{n s}=0.032$ gives $\varepsilon_{0}=\delta_{n s} / 2=0.016$. Hence this class of models predicts $r=0.256$, which is larger than permitted by the BPK-data.

The potential is given as a function of the inflaton field by Equation (3.2) and as a function of the number of e-foldings by performing the integral in Equation (5.4), giving

$$
V(N)=V_{0} e^{2 \varepsilon_{0} N}
$$

From Equation (5.7) we have in this case

$$
N=\sqrt{\kappa / 2 \varepsilon_{0}}\left(\phi-\phi_{f}\right)
$$

and hence that

$$
V(\phi)=V_{f} e^{\sqrt{2 \kappa \varepsilon_{0}}\left(\phi-\phi_{f}\right)}
$$

\subsection{Perturbative Class}

In this class of models $\varepsilon$ is given by a power function of $N$,

$$
\varepsilon=\beta / N^{n}
$$

A similar parametrization has been considered by Huang [43] and by Lin, Gao and Gong [72]. This parametrization is not meant to describe the end of the inflationary models when $N=0$, but represents the slow roll era with $N>>1$.

It will be shown below that for $n<<N, \beta=n / 4, n=1$ this corresponds respectively to polynomial chaotic inflation, brane inflation, tachyon inflation, DBI-inflation and loop inflation, for $n=$ $4 / 3$ to arctan inflation, for $n=3 / 2$ to inflation with fractional potential, for $n=2$ to Hilltop, mutated Hilltop and Kähler moduli inflation, and with $N>>1$ to Higgs inflation and supergravity motivated inflation, and for $n=3$ approximately to Coleman-Weinberg inflation (see Table 1 ).

Table 1. Values of spectroscopic parameters according to the chaotic inflation model.

\begin{tabular}{cccccc}
\hline$n$ & $n_{S}$ & $n_{T}$ & $r$ & $\alpha_{s}$ & $\alpha_{T}$ \\
\hline $2 / 3$ & 0.973 & -0.007 & 0.045 & -0.0005 & -0.00013 \\
1 & 0.97 & -0.01 & 0.08 & -0.0006 & -0.0002 \\
2 & 0.96 & -0.02 & 0.16 & -0.0008 & -0.0004 \\
3 & 0.95 & -0.03 & 0.24 & -0.0010 & -0.0006 \\
4 & 0.94 & -0.04 & 0.32 & -0.0012 & -0.0008 \\
\hline
\end{tabular}

Combining Equation (3.52) with Equation (5.12) we have

$$
\phi, N=M_{P} \sqrt{2 \beta} N^{-n / 2} \text {. }
$$

Integrating with $\phi(1)=\phi_{f}$ gives

$$
\phi(N)=\left\{\begin{array}{ll}
\phi_{f}+\frac{2 \sqrt{2 \beta} M_{P}}{2-n}\left(N^{1-n / 2}-1\right)=\phi_{f}+\frac{2 \sqrt{2 \beta} M_{P}}{n-2}\left[1-(1 / N)^{n / 2-1}\right], & n \neq 2 \\
\phi_{f}+M_{P} \sqrt{2 \beta} \ln N & n=2
\end{array} .\right.
$$


Note that increasing $N$ means going backwards in time, since $\phi(N)$ is the value of the inflaton field $N$ e-folds before the end of the slow roll era. Hence the fact that $\phi(N)$ increases with $N$ means that the inflaton field decreases with time.

At a large part of the slow roll era $N>>1$. Then the expressions for the inflaton field can be approximated by

$$
\phi(N)= \begin{cases}\frac{2 \sqrt{2 \beta} M_{P}}{2-n} N^{1-n / 2}, & 0<n<2 \\ \frac{2 \sqrt{2 \beta} M_{P}}{n-2}, & n>2 \\ M_{P} \sqrt{2 \beta} \ln N, & n=2\end{cases}
$$

It follow from Equations (3.20) and (3.53) that to lowest order

$$
\varepsilon \approx \varepsilon_{H}=(\ln H)_{N}
$$

Equations (2.16), (5.16) and (5.12) give

$$
(\ln V)_{N}=2(\ln H)_{N}=2 \varepsilon=\frac{2 \beta}{N^{n}} .
$$

Integration with $V(0)=0$ gives

$$
V(N)= \begin{cases}V_{0} N^{2 \beta}, & n=1 \\ V_{0}-V_{0} e^{-\frac{2 \beta}{n-1}\left(\frac{1}{N}\right)^{n-1}}, & n>1 .\end{cases}
$$

From Equations (5.15) and (5.18) we obtain

$$
\begin{gathered}
V(\phi)= \begin{cases}V_{0} \phi^{4 \beta}, & n=1 \\
V_{0}-V_{0} \exp \left[-2 \beta \exp \left(\frac{\phi}{M_{P} \sqrt{2 \beta}}\right)\right], & n=2 \\
V_{0}-V_{0} \exp \left[-\frac{2 \beta}{n-1}\left(\frac{\phi}{M}\right)^{p}, p=\frac{2(1-n)}{2-n}, \quad M=\frac{2 \sqrt{2 \beta}}{2-n} M_{P},\right. & n>1, n \neq 2\end{cases} \\
V(\phi)=\left\{\begin{array}{ll}
V_{0} \phi^{4 \beta}, & n=1 \\
V_{0}-V_{0} \exp \left[-2 \beta \exp \left(\frac{\phi}{M_{P} \sqrt{2 \beta}}\right)\right], & n=2 \\
V_{0}-V_{0} \exp \left[-\frac{2 \beta}{n-1}\left(\frac{\phi}{M}\right)^{p}, \quad p=\frac{2(1-n)}{2-n}, \quad M=\frac{2 \sqrt{2 \beta}}{2-n} M_{P},\right. & n>1, n \neq 2
\end{array} .\right.
\end{gathered}
$$

The spectral parameters as calculated from Equations (5.1), (5.2), and (5.12) are to lowest order in $N$,

$$
\left.\begin{array}{l}
\delta_{n s}=\frac{2 \beta+1}{N}, \quad r=\frac{16 \beta}{N}, \quad n_{T}=-\frac{2 \beta}{N}, \quad \alpha_{S}=-\frac{2 \beta+1}{N^{2}}, \quad \alpha_{T}=-\frac{2 \beta}{N^{2}}, \quad n=1 \\
\delta_{n s}=\frac{n}{N}+\frac{2 \beta}{N^{n}}, \quad r=\frac{16 \beta}{N^{n}}, \quad n_{T}=-\frac{2 \beta}{N}, \quad \alpha_{S}=-\frac{n}{N^{2}}\left(1+\frac{2 \beta}{N^{n-1}}\right), \quad \alpha_{T}=-\frac{2 \beta}{N^{2}}, \quad n>1
\end{array}\right\} .
$$

The expressions (5.20) imply the following relationships

$$
\left.\begin{array}{l}
r=\frac{16 \beta}{1+2 \beta} \delta_{n s}=8\left(\delta_{n s}-\frac{1}{N}\right), \quad \alpha_{S}=-\frac{\delta_{n s}^{2}}{1+2 \beta}, \alpha_{T}=-\frac{2 \beta}{(1+2 \beta)^{2}} \delta_{n s}^{2}, N=-\frac{\delta_{n s}}{\alpha_{S}}, \quad n=1 \\
r=8\left(\delta_{n s}-\frac{n}{N}\right), \quad n_{T}=\frac{\beta}{4 n}\left(r-8 \delta_{n s}\right), \quad n=N\left(\delta_{n s}-\frac{r}{8}\right), \quad n>1
\end{array}\right\},
$$

or

$$
\left.\begin{array}{l}
\beta=\frac{r}{2\left(8 \delta_{n s}-r\right)} \quad, \quad n=1 \\
\beta=\frac{4 n n_{T}}{r-8 \delta_{n s}} \quad \text { or } \quad \beta=\frac{1}{2} N^{n}\left(\delta_{n s}-\frac{n}{N}\right)=N^{n} \frac{r}{16} \quad, \quad n>1
\end{array}\right\} .
$$

With $\delta_{n s}=0.032, r=0.10$ and $N=50$ we get from the last expressions in Equations (5.21) and (5.22) $n=1.0$ and $\beta=0.31$, showing that for this class of models polynomial inflation is favored. Furthermore these values of $\delta_{n s}$ and $N$ gives $\alpha_{S}=-0.0006$. This class of inflationary models has been considered by L. Barranco, L. Boubekeur and O. Mena [73] with $N$ replaced by $N+1$, and by Gao and Gong [74] with $n=2$ and $N$ replaced by $N+N_{0}$. 
A related parametrization has been considered by Lin, Gao and Gong [72] and Q. Fei et al. [75],

$$
\delta_{n s}=\frac{p}{N+\alpha}
$$

The same parametrization with $\alpha=0$ has been discussed by P. Creminelli et al. [76], R. Gobetti et al. [77]. Here the constant $\alpha$ accounts for the contribution from the scalar field $\phi_{e}=\phi(0)$ at the end of inflation where $N=0$. Inserting Equation (5.24) into the first of Equation (5.1) gives

$$
\varepsilon^{\prime}=\varepsilon\left(2 \varepsilon-\frac{p}{x}\right)
$$

where $x=N+\alpha$. Introducing $y=1 / \varepsilon$ Equation (5.25) may be written

$$
\left(x^{-p} y\right)^{\prime}=-2 x^{-p}
$$

From the solution of this equation with $\varepsilon(0)=1$ and $\alpha \neq 0$ together with $r=16 \varepsilon$ we get

$$
r=\left\{\begin{array}{ll}
\frac{16(p-1)}{\frac{\left(1+\frac{N}{\alpha}\right)\left[2 \alpha+(p-1-2 \alpha)\left(1+\frac{N}{\alpha}\right)^{p-1}\right]}{16}}, & p \neq 1, \\
\frac{16}{\left(1+\frac{N}{\alpha}\right)\left[1-2 \alpha \ln \left(1+\frac{N}{\alpha}\right)\right]} & , \quad p=1
\end{array} .\right.
$$

Since $\varepsilon>0$ and the last term in the denominator dominates, the constant $p$ must fullfill $p \geq 1+2 \alpha$ for $p \neq 1$. In the case $p=1$ we must have $\alpha>0$. From Equation (5.24) this requires $N \delta_{n s}<1$. However $N=50, \delta_{n s}=0.032$ gives $N \delta_{n s}=1.6$ so this case is in conflict with the Planck data and the requirements of a sufficiently long inflationary era to solve the horizon problem. Combining Equations (5.24) and (5.27) gives

$$
r=\frac{16(p-1) \delta_{n s}}{2 p+\left[2\left(N \delta_{n s}-p\right)+(p-1) \delta_{n s}\right]\left(\frac{p}{N \delta_{n s}-p}\right)^{p}} .
$$

In order to have $r>0$ the constant $p$ must be restricted to $1<p<N \delta_{n s}$ unless $p=1+2 \alpha$. Using Equation (5.24) we have

$$
\alpha=\frac{p-N \delta_{n s}}{\delta_{n s}},
$$

So in the special case that $p=1+2 \alpha$ we have

$$
p=\frac{(2 N-1) \delta_{n s}}{2-\delta_{n s}}
$$

Hence

$$
2\left(N \delta_{n s}-p\right)=-(p-1) \delta_{n s}=-2 \alpha \delta_{n s}<0
$$

So $p>N \delta_{n s}$, but in this case this is allowed because now the last term in the denominator of the first Equation (5.28) vanishes.

The tensor-to-scalar ratio $r$ is plotted as a function of $p$ from Equation (5.28) in Figure 1 for $N=60, \delta_{n s}=0.032$ which gives $N \delta_{n s}=1.92$.

We see from Figure 1 that for inflationary models with a scalar tilt given in terms of the $N$-fold by an equation of the form (5.24), the present 'standard' values of the number of $N$-folds and the scalar tilt, $N=60, \delta_{n s}=0.032$, lead to acceptable values of the tensor-to-scalar ratio, $r<0.03$. 


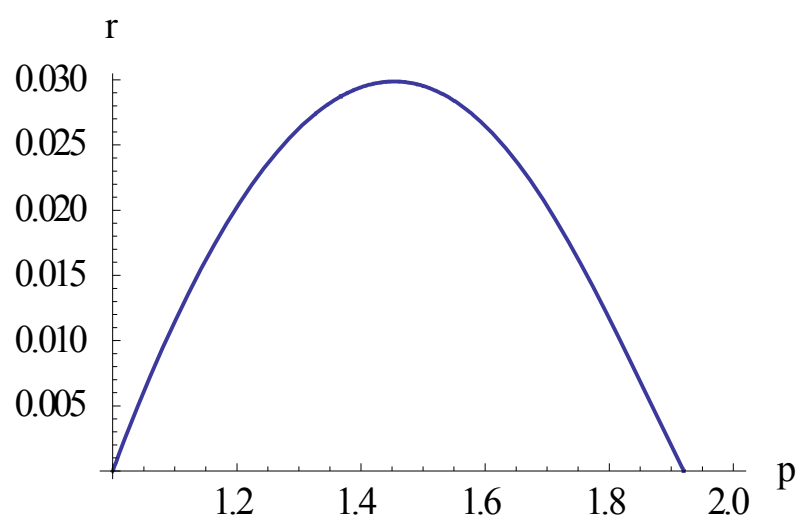

Figure 1. The tensor-to-scalar-ratio plotted as a function of $p$ for an inflationary model with $N=60$, $\delta_{n s}=0.032$.

Equations (5.4) and (5.27) now give the potential as a function of the number of $N$-folds,

$$
V(N)=\frac{\left(1+\frac{N}{\alpha}\right)^{p-1}}{\left(1-\frac{2 \alpha}{p-1}\right)\left(1+\frac{N}{\alpha}\right)^{p-1}+\frac{2 \alpha}{p-1}} V(0)
$$

where $V(0)=1 /(p-1)$.

For the special case $p=1+2 \alpha$ Equation (5.27) for the tensor to scalar ratio gives

$$
r=\frac{8 \alpha}{N+\alpha} .
$$

Using once more Equation (5.24) we get

$$
r=\frac{8(p-1)}{p} \delta_{n s}
$$

which may be written

$$
p=\frac{8 \delta_{n s}}{8 \delta_{n s}-r} .
$$

The Planck results $\delta_{n s}=0.032, r<0.04$ give $p<1,016$ corresponding to $\alpha<0.008$. In this case Equation (5.32) reduces to

$$
V(N)=V(0)\left(1+\frac{N}{\alpha}\right)^{2 \alpha} .
$$

Inserting Equation (5.33) into Equation (5.7) and integrating gives

$$
\phi(N)=\phi(0)+2 \sqrt{2} M_{P} \alpha\left(\sqrt{1+\frac{N}{\alpha}}-1\right)
$$

Hence

$$
V(\phi)=V_{0}\left(\phi-\phi_{0}\right)^{4 \alpha}
$$

where

$$
V_{0}=V(0) / 2 \sqrt{2} M_{P} \alpha, \phi_{0}=\left(\phi(0) / 2 \sqrt{2} M_{P} \alpha\right)-1 .
$$

If the constant $\phi_{0}$ is chosen to be zero, the constant $\alpha$ is represents the potential at the end of inflation as follows

$$
\alpha=\phi_{e} / 2 \sqrt{2} M_{p} .
$$


With $N>50$ we have $\phi>M_{P}$ so this model corresponds to power law large field inflation. Lin, Gao and Gong [72] have shown that this model is only marginally compatible with the Planck results.

Koh et al. [78] have considered a class of models with

$$
\delta_{n s}=\frac{2}{N+\alpha} \quad, \quad r=\frac{q}{N^{2}+\gamma N+\alpha} .
$$

This corresponds to $p=2$ in Equation (5.24).

\subsection{Reconstructing the Inflaton Potential from the Spectral Parameters}

T. Chiba [69] has shown how one can find the inflaton potential from the spectral index. The formalism has recently been generalized to inflationary models with a Gauss-Bonnet term by Koh et al. [78].

Using Equations (3.1) and (3.51) we have

$$
V^{\prime}=\frac{1}{M_{P}}(V V, N)^{1 / 2} .
$$

Differentiating once more we get

$$
V^{\prime \prime}=\frac{V V,_{N}}{M_{P}^{2} V^{\prime}}=\frac{V}{M_{P}^{2}} \frac{1}{\left(V V,_{N}\right)^{1 / 2}}\left[\left(V V,_{N}\right)^{1 / 2}\right],_{N}=\frac{V}{2 M_{P}^{2}}\left[\ln V V,_{N}\right]_{N} .
$$

Hence, the slow roll parameters $\varepsilon$ and $\eta$ are

$$
\varepsilon=\frac{1}{2}(\ln V)_{N} \quad, \quad \eta=\frac{1}{2}[\ln (V V, N)]_{N} .
$$

This, together with the definitions (4.4), (4.13) and (4.29), gives

$$
\delta_{n s}=\left\{\ln \left[\left(-\frac{1}{V}\right),_{N}\right]\right\}, \quad r=8(\ln V)_{, N} \quad, \quad \alpha_{S}=\delta_{n s, N} .
$$

The potential as a function of $N$ is given by the first of these equations. It can be written

$$
V(N)=-1 / \int e^{-\int \delta_{n s}(N) d N} d N .
$$

Knowing $V$ as a function of $N$ the relationship between $\phi$ and $N$ is found by integrating Equation (3.56) in the form

$$
\phi=M_{P} \int \sqrt{(\ln V)_{N}} d N=\frac{M_{P}}{2 \sqrt{2}} \int \sqrt{r(N)} d N .
$$

Chiba [69] has illustrated the method by considering a class of inflationary models where

$$
\delta_{n s}=2 / N \text {. }
$$

This corresponds to the parametrization (5.24) with $p=2, \alpha=0$. With $N=60$ this gives $\delta_{n s}=0.033$ in agreement with the Planck data. Inserting this into equation the first of Equation (5.46) gives

$$
V(N)=\frac{N}{\alpha+\beta N}
$$


where $\alpha$ and $\beta$ are constants of integration. Inserting this into the two last equations in (5.45) and introducing $\gamma \equiv \beta / \alpha$ gives

$$
r=\frac{8}{N(1+\gamma N)} \quad, \quad \alpha_{S}=-\frac{2}{N^{2}} .
$$

$N=60$ gives $\alpha_{S}=-0.0006$ which is allowed by the Planck data. For this model the $r, \delta_{n s}$-relation, and the corresponding relations involving $\alpha_{s}$, are

$$
r=\frac{4 \delta_{n s}^{2}}{\delta_{n s}+2 \gamma} \quad, \quad \alpha_{s}=-\frac{r}{6}=-\frac{1}{2} \delta_{n s}^{2} .
$$

From the first of these equations we get

$$
\gamma=\frac{1}{2}\left(4 \frac{\delta_{n s}}{r}-1\right) \delta_{n s} .
$$

Inserting $\delta_{n s}=0.032$ and $r<0.04$ gives $\gamma>0.035$.

Inserting (5.49) into Equation (5.42) gives

$$
\phi=\int \frac{d N}{\sqrt{\gamma N^{2}+N}} .
$$

Integration with $N(0)=0$ leads to

$$
N= \begin{cases}(1 / \gamma) \sinh ^{2}\left(\frac{1}{2} \sqrt{\gamma} \phi\right), & \gamma>0 \\ \phi^{2} / 4 & , \quad \gamma=0 \\ (1 / \gamma) \sin ^{2}\left(\frac{1}{2} \sqrt{-\gamma} \phi\right), & \gamma<0\end{cases}
$$

Inserting these expressions into Equation (5.46) gives

$$
V(\phi)=\left\{\begin{array}{ll}
(1 / \beta) \tanh ^{2}((1 / 2) \sqrt{\gamma} \phi), & \gamma>0 \\
\phi^{2} / 4 \alpha & , \quad \gamma=0 \\
-(1 / \beta) \tan ^{2}((1 / 2) \sqrt{-\gamma} \phi), & \gamma<0
\end{array} .\right.
$$

Inflationary universe models with these potentials for the inflaton field will be considered in detail later in this article and have also been studied by Kallosh and Linde [79]. The case $\beta>0$ gives inflationary models of the type motivated by supergravity, for example so-called $\alpha$ - attractor models, and will be considered in Section 6.11. The case $\beta=0$ represents the simplest chaotic inflationary models and will be presented in Section 6.1. The case $\beta<0$ will also be considered in Section 6.11.

\subsection{S-Dual and Hyperbolic Inflation}

Lin, Gao and Gong [72] have also considered the parametrization

$$
\varepsilon(N)=\frac{\alpha}{1+s e^{-\beta N}},
$$

where $s= \pm 1$ with $\alpha>0, \beta>0$. For this class of inflationary models

$$
\delta_{n s}=\frac{2 \alpha-\beta s e^{-\beta N}}{1+s e^{-\beta N}} \quad, \quad r=\frac{16 \alpha}{1+s e^{-\beta N}} .
$$

It follows from these equations that

$$
r=\frac{16 \alpha\left(\delta_{n s}+\beta\right)}{2 \alpha+\beta} .
$$


and that

$$
8 s \beta=\left(r-8 \delta_{n s}\right)\left(s+e^{\beta N}\right) .
$$
$s=-1$.

Inserting the Planck values $\delta_{n s}=0.032, r<0.04$ gives $r-8 \delta_{n s}<0$. Hence $\beta>0$ requires

Inserting the first of the expressions (5.56) into Equation (5.45) and performing the integration gives

$$
V(N)=C\left(s+e^{\beta N}\right)^{2 \alpha / \beta},
$$

where $C$ is a constant. Inserting the expression (5.59) into Equation (5.46) and integrating gives

$$
\left.\begin{array}{ll}
\phi=\phi_{0}+\frac{2 \sqrt{2 \alpha}}{\beta} \operatorname{arsinh}\left(e^{\beta N / 2}\right), & s=1 \\
\phi=\phi_{0}+\frac{2 \sqrt{2 \alpha}}{\beta} \operatorname{arcosh}\left(e^{\beta N / 2}\right), & s=-1
\end{array}\right\},
$$

Inserting this into Equation (5.59) gives

$$
\left.\begin{array}{ll}
V(\phi)=V_{0} \cosh ^{4 \alpha / \beta}\left(\frac{\beta \phi}{2 \sqrt{2 \alpha}}\right), & s=1 \\
V(\phi)=V_{0} \sinh ^{4 \alpha / \beta}\left(\frac{\beta \phi}{2 \sqrt{2 \alpha}}\right), & s=-1
\end{array}\right\} .
$$

Lin, Gao and Gong [72] have shown that these inflationary models do not satisfy the Planck/BICEP2 constraints at the $99.8 \%$ confidence level. It may be shown, for example, that with the Planck values for $\delta_{n s}, r$ and $N$ Equation (5.58) has no positive, real solution for $\beta$.

The same authors have also investigated some inflationary models with the parametrization

$$
\phi(N)=\mathcal{K}^{-1 / 2}\left(N+N_{0}\right)^{\sqrt{2 \gamma}},
$$

where $N_{0}$ is a positive constant and I have replaced their $\beta$ by $\sqrt{2 \gamma}$ to simplify later expressions. Equation (5.46) may be written

$$
V(N)=\exp \left\{\kappa \int\left[\phi^{\prime}(N)\right]^{2} d N\right\} .
$$

For $\gamma \neq 0, \gamma \neq 1 / 8$ integration of this equation gives

$$
V(N)=C \exp \left[\frac{2 \gamma}{2 \sqrt{2 \gamma}-1}\left(N+N_{0}\right)^{2 \sqrt{2 \gamma}-1}\right] .
$$

Inserting Equation (5.62) gives

$$
V(\phi)=K \exp \left(\frac{\kappa^{1 / \sqrt{2 \gamma}} 2 \gamma}{2 \sqrt{2 \gamma}-1} \phi^{2-1 / \sqrt{2 \gamma}}\right) .
$$

This leads to

$$
\delta_{n s}=\frac{2 \gamma}{\left(N+N_{0}\right)^{p}}+\frac{p}{N+N_{0}} \quad, \quad r=\frac{16 \gamma}{\left(N+N_{0}\right)^{p}},
$$

where $p=2(1-\sqrt{2 \gamma})$. The usual condition for a graceful exit of the slow roll era is $\varepsilon(0)=1$, hence $r(0)=16$, which gives $\gamma=N_{0}^{p}$. Equation (5.66) gives the $\delta_{n s}, r$ relationship

$$
\delta_{n s}=\frac{r}{8}+\frac{p}{(16 \gamma)^{1 / p}} r^{1 / p}
$$

The Planck values $\delta_{n s}=0.032, r=0.05$ have $\delta_{n s}>r / 8$ which requires $\gamma<1 / 2$. Assuming that $p=1$ and solving Equation (5.67) with these values of $\delta_{n s}$ and $r$, give $\gamma=r / 2\left(8 \delta_{n s}-r\right) \approx 0.12$. In this 
case $N_{0} \approx(2 / r)-N$. A positive value of $N_{0}$ requires $r<2 / N$. For $N=60$ this leads to the prediction $r<0.033$.

Lin, Gao and Gong [72] have furthermore considered the parametrization

$$
\phi(N)=\beta \ln \left(N+N_{0}\right)
$$

This leads to

$$
V(N)=K \exp \left(-\frac{\beta^{2}}{N+N_{0}}\right)
$$

and

$$
V(\phi)=K \exp \left[-\beta^{2} \exp (-\phi / \beta)\right]
$$

Hence,

$$
\delta_{n s}=\frac{2}{N+N_{0}}+\frac{\beta^{2}}{\left(N+N_{0}\right)^{2}} \quad, \quad r=\frac{8 \beta^{2}}{\left(N+N_{0}\right)^{2}} .
$$

This is similar to Equation (5.66) with $p=2$. In this case the $\delta_{n s}, r$ relationship takes the form

$$
\delta_{n s}=\frac{r}{8}+\frac{1}{\beta} \sqrt{\frac{r}{2}}
$$

Thus, $\beta$ can be expressed in terms of $\delta_{n s}$ and $r$ as

$$
\beta=\frac{\sqrt{8 r}}{8 \delta_{n s}-r}
$$

Inserting the Planck/BICEP2 data gives $\beta<7.2$.

Finally Lin, Gao and Gong [72] considered the parametrization

$$
\phi(N)=\exp \left(\beta N+N_{0}\right)
$$

with $\beta<0$. Then

$$
V=V_{0} \exp \left(\beta \phi^{2} / 2\right)
$$

giving

$$
\delta_{n s}=\beta^{2} \exp \left(2 \beta N+2 N_{0}\right)-2 \beta, \quad r=8 \beta^{2} \exp \left(2 \beta N+2 N_{0}\right) .
$$

The $\delta_{n s}, r$ relationship can then be written

$$
\beta=\frac{1}{16}\left(r-8 \delta_{n s}\right) .
$$

Here $\delta_{n s}=0.032, r<0.04$ gives $\beta<-0.016$.

If the end of the slow roll era is defined by $\varepsilon(N=0)=1$, i.e., $r(N=0)=16$ we get $\beta^{2} \exp \left(2 N_{0}\right)=2$ giving

$$
\delta_{n s}=2\left(e^{2 \beta N}-\beta\right), \quad r=16 e^{2 \beta N} .
$$

It follows from Equation (5.78) that

$$
N=\frac{8}{8 \delta_{n s}-r} \ln \left(\frac{16}{r}\right)
$$

With $\delta_{n s}=0.032$ this function has a minimum for $r_{1}=0.036$ giving $N\left(r_{1}\right)=222$. Hence this class of inflationary models has $N>222$, which is not a realistic scenario. 


\subsection{The Equation of State Parameter during the Slow Roll Era}

We shall now follow Mukhanov [30]. Equation (4.5) can be written

$$
r=24(1+w)
$$

Inserting Equation (3.57) into Equation (4.13) leads to

$$
\delta_{n s}=3(1+w)-(\ln w)_{N} .
$$

The quantity $1+w$ must have a small value during the inflationary era, but not zero. A zero value means that the dominating fluid is LIVE which has constant density during the exponentially expanding era which can be represented in Einstein's field equations by a cosmological constant. This does not provide any mechanism for a graceful exit from the inflationary era. One needs a time dependent equation of state parameter. Mukhanov [30] writes that from the very beginning of the inflationary era there should be a small deviation of the value of $1+w$ from zero, and the value of $1+w$ should be monotonously increasing during the inflation, ending with an absolute value of order 1 at the end of the era.

The usual condition for a graceful exit of the slow roll era is to require that $\varepsilon=r / 16=1$ at the end of inflation. This implies that $1+w=2 / 3$ at the end of inflation. Mukhanov has therefore proposed the ansatz

$$
1+w=\frac{\beta}{(N+1)^{\alpha}}
$$

where $\alpha$ and $\beta$ are positive constants of order unity. The condition $1+w=2 / 3$ at the end of inflation with $N=0$ gives $\beta=2 / 3$. Gao and Gong [74] have considered inflationary models obeying the ansatz (5.82) with $\alpha=2$ and $N+1$ replaced by $N+N_{0}$.

Equations (5.80)-(5.82) give

$$
\delta_{n s}=\frac{3 \beta}{(N+1)^{\alpha}}+\frac{\alpha}{N+1} \quad, \quad r=\frac{24 \beta}{(N+1)^{\alpha}} .
$$

The condition $\varepsilon(0)=1$ at the end of inflation requires $\beta=2 / 3$.

We have the following cases

$$
\delta_{n s} \approx \begin{cases}\frac{3 \beta+1}{N+1}, \quad r=\frac{24 \beta}{3 \beta+1} \delta_{n s}, & \alpha=1, \\ \frac{\alpha}{N+1}, \quad r=24 \beta\left(\frac{\delta_{n s}}{\alpha}\right)^{\alpha}, & \alpha>1, \\ \frac{3 \beta}{(N+1)^{\alpha}}, & r=8 \delta_{n s}, \quad \alpha<1 .\end{cases}
$$

It follows from Equation (5.83) that

$$
\alpha=\left(\delta_{n s}-\frac{r}{8}\right)(N+1)
$$

independent of the value of $\beta$. A positive value of $r$ requires $\alpha<(N+1) \delta_{n s}$. Inserting $\delta_{n s}=0.032$, $N=60$ gives $\alpha<1.95$, while $\delta_{n s}=0.032, r=0.05, N=60$ in Equation (5.85) gives $\alpha=1.6$, and inserting this value into the second of Equation (5.83) gives $\beta=1.3$ which is a little higher than required by the condition for a graceful exit of the slow roll era. Also the value of $\alpha$ is smaller than that assumed by Gao and Gong [74].

However replacing $N+1$ by $N+N_{0}$ permits the choice $\alpha=2$ of Gao and Gong. Then the modified Equation (5.85) gives

$$
N_{0}=\frac{16}{8 \delta_{n s}-r}-N
$$

Hence $r>0$ implies $N_{0}>2.5$, and $\delta_{n s}=0.032, r=0.05, N=60$ gives $N_{0}=17.7$. 
As an illustration, choosing $\beta=1 / 3$ in Equation (5.83) gives

$$
\delta_{n s}=\frac{r}{8}+\left(\frac{r}{8}\right)^{1 / \alpha} \alpha .
$$

Since $|r|<<1$ the first term dominates for $\alpha<1$ and the second one for $\alpha>1$. Note that the value $\alpha=1$ gives $r=4 \delta_{n s}=0.128$ which is larger than allowed by the BICEP2/Planck result. Also as seen from Equation (5.84), $\alpha<1$ gives too high value of $r$. The parameter $\delta_{n s}$ is plotted as a function of $\alpha$ for $r=0.05$ in Figure 2.

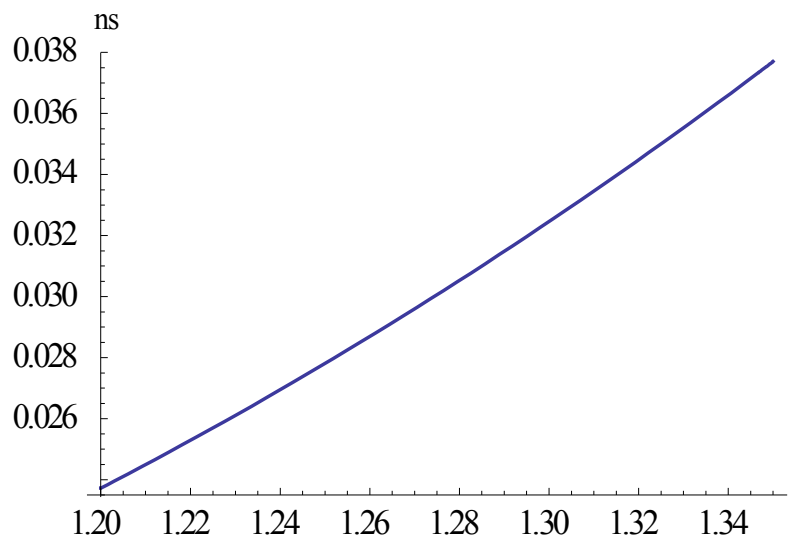

Figure 2. The parameter $\delta_{n s}$ plotted as a function of $\alpha$ for $1.20<\alpha<1.35$ with $r=0.05$.

Gao and Gong [74] have applied the $N$-formalism and deduced the potential with the parametrization

$$
\varepsilon=\frac{3 \beta}{2\left(N+N_{0}\right)^{2}} .
$$

At the end of inflation $\varepsilon(0)=1$ which leads to

$$
N_{0}=\sqrt{\frac{3 \beta}{2}} \quad \text { or } \quad \beta=\frac{2}{3} N_{0}^{2} .
$$

Inserting the expression (5.86) into (5.89) gives

$$
\beta=\frac{2}{3}\left(\frac{16}{8 \delta_{n s}-r}-N\right)^{2} .
$$

The value $\beta=2 / 3$ required by graceful exit of inflation, gives $N_{0}=1$ and

$$
r=8\left(\delta_{n s}-\frac{2}{N+1}\right) .
$$

Hence with the values $\delta_{n s}=0.032, N=60$ this class of models predicts $r \approx 0$. A positive value of $r$ requires

$$
N>\frac{2}{\delta_{n s}}-1
$$

Thus $\delta_{n s}=0.032$ gives $N>61.5$.

Inserting the parametrization (5.88) into the first of Equations (5.44) and (5.47) and integrating gives

$$
V(N)=V_{0} \exp \left(-\frac{3 \beta}{N+N_{0}}\right) .
$$


Together with Equation (5.45) this gives

$$
\delta_{n s}=\frac{2}{N+N_{0}}+\frac{3 \beta}{\left(N+N_{0}\right)^{2}}
$$

and

$$
\phi=\phi_{0}+\sqrt{3 \beta} \ln \left(1+\frac{N}{N_{0}}\right), \quad \phi_{0}=\sqrt{\frac{3 \beta}{4}} \ln \frac{3 \beta}{2} .
$$

The graceful exit value $\beta=2 / 3$ and $N_{0}=1$ gives $\phi_{0}=0$ and

$$
\phi=\sqrt{2} \ln (N+1)
$$

It follows from Equations (5.93) and (5.95) that for this class of inflationary models the potential is

$$
V(\phi)=V_{0} \exp \left(-2 e^{-\phi}\right) .
$$

Myrzakulov et al. [29] have followed up the analysis of Mukhanov [30] and investigated how one can construct viable inflationary models starting from the measured values of $n_{S}$ and $r$ and assuming the ansatz (5.82). In particular they have considered the cases $\alpha=1$ and $\alpha=2$.

From the second of Equation (2.9) we have

$$
1+w=\frac{2 \dot{\phi}^{2}}{\dot{\phi}^{2}+2 V} .
$$

Myrzakulov et al. [29] assume that

$$
1+w=\beta\left(\rho_{0} / \rho\right)^{1 / 3 \beta}
$$

where $\rho_{0}$ is the energy density at the end of inflation. From Equations (2.1), (5.82) and (5.83) we have

$$
\dot{\phi}^{2}=(\beta / 2)\left(2 \rho_{0}\right)^{1 / 3 \beta}\left(\dot{\phi}^{2}+2 V\right)^{1-\frac{1}{3 \beta}} .
$$

With the slow roll approximation $\dot{\phi}^{2}<<V$ this equation reduces to

$$
\dot{\phi}=\frac{\sqrt{\beta} \rho_{0}^{1 / 6 \beta}}{V^{\frac{1-3 \beta}{6 \beta}}} .
$$

Equation (3.4) can be written

$$
V^{-1 / 2} V^{\prime}=\sqrt{3} \kappa \dot{\phi}
$$

Inserting the expression (5.101) for $\dot{\phi}$ one obtains

$$
V^{\frac{1}{6 \beta}-1} V^{\prime}=\sqrt{3 \beta} \kappa \rho_{0}^{1 / 6 \beta} .
$$

Integrating with $V(0)=0$ leads to

$$
V(\phi)=\left(\frac{3 \kappa^{2}}{\beta}\right)^{3 \beta} \rho_{0}\left(\frac{\phi}{6}\right)^{6 \beta}
$$

The preferred value of $\beta$ is $\beta=1 / 3$ which gives

$$
V(\phi)=(\kappa / 2)^{2} \rho_{0} \phi^{2} .
$$


Following Davis et al. [80] we shall now deduce some general results for inflationary models where the inflaton field has a potential of the form

$$
V(\phi)=V_{0}[1-f(\widetilde{\phi})] \quad, \quad \widetilde{\phi}=\phi / \phi_{0} .
$$

Assuming that $f(\widetilde{\phi})<<1$ during the slow roll era we may approximate $\varepsilon, \eta$ and $\xi$ by

$$
\varepsilon \approx \frac{1}{2 \kappa}\left(\frac{V^{\prime}}{V_{0}}\right)^{2}, \quad \eta \approx \frac{1}{\kappa} \frac{V^{\prime \prime}}{V_{0}}, \quad \xi \approx \frac{1}{\kappa^{2}} \frac{V^{\prime} V^{\prime \prime \prime}}{V_{0}^{2}} .
$$

The expressions for the standard slow roll parameters then take the form

$$
\varepsilon \simeq \frac{M_{P}^{2}}{2 \phi_{0}^{2}}\left[f^{\prime}(\widetilde{\phi})\right]^{2} \quad, \quad \eta \simeq \frac{M_{P}^{2}}{\phi_{0}^{2}} f^{\prime \prime}(\widetilde{\phi}) \quad, \quad \xi \simeq \frac{M_{P}^{4}}{\phi_{0}^{4}} f^{\prime}(\widetilde{\phi}) f^{\prime \prime \prime}(\widetilde{\phi})
$$

This gives

$$
\delta_{n s} \simeq \frac{M_{P}^{2}}{\phi_{0}^{2}}\left(3 f^{\prime 2}-2 f^{\prime \prime}\right) \quad, \quad r=8 \frac{M_{P}^{2}}{\phi_{0}^{2}} f^{\prime 2} \quad, \quad \xi=-2 \frac{M_{P}^{4}}{\phi_{0}^{4}}\left(3 f^{\prime 4}-4 f^{\prime 2}+f^{\prime} f^{\prime \prime \prime}\right) .
$$

The number of $N$-folds during the slow roll era is

$$
N=-\frac{\phi_{0}^{2}}{M_{P}^{2}} \int_{\widetilde{\phi}_{f}}^{\widetilde{\phi}} \frac{d \widetilde{\phi}}{f^{\prime}(\widetilde{\phi})}
$$

It is usual to define the end of the slow roll era by the condition that $\varepsilon\left(\widetilde{\phi}_{f}\right)=1$. Hence the value of the inflaton field at the end of the slow roll era is given by

$$
f^{\prime}\left(\widetilde{\phi}_{f}\right)=\sqrt{2} \phi_{0} / M_{P}
$$

Finally we shall consider implications of a vanishing tensor-to-scalar-ratio. Biagetti et al. [81] asked "What We Can Learn from the Running of the Spectral Index if no Tensors are Detected in the Cosmic Microwave Background Anisotropy". Here we shall consider the implications of a vanishing value of $r$ in general for the inflationary models.

It follows immediately from Equation (4.4) that $\varepsilon=0$ if $r=0$ and hence, from Equation (4.15), $n_{T}=0$. From the first of the Equation (3.1) it then follows that the potential $V$ has an extremum at the horizon crossing. It cannot be constant because that leads to a scale invariant spectrum, $\delta_{n s}=0$, which is not allowed by the Planck data.

With $r=0$ Equations (4.13) and (4.29) give

$$
\delta_{n s}=-2 \eta \quad \alpha_{S}=-2 \xi
$$

Since the Planck data give $\delta_{n s}>0$ this equation implies that $\eta<0$. Hence the second derivative of the potential must be negative, and the potential has a maximum at horizon crossing. Also there will be a running of the scalar spectral index only if the third derivative of the potential is non vanishing.

\subsection{The $\beta$-Function Formalism}

This formalism was introduced in 2015 by P. Binétruy and coworkers [82]. It was inspired by the fact that the dynamical equations of the inflationary models can be given a form similar to the renormalization group equations. 
They defined a new function

$$
\beta(\phi)=-\frac{2}{\kappa} \frac{H^{\prime}}{H} .
$$

Combining this with Equation (2.12) we obtain

$$
\dot{H}=-(\kappa / 2) H^{2} \beta^{2}(\phi) .
$$

Hence the acceleration of the scale factor is

$$
\ddot{a}=a\left(H^{2}+\dot{H}\right)=a H^{2}\left[1-\frac{\kappa}{2} \beta^{2}(\phi)\right],
$$

showing that accelerated expansion requires $|\beta|<\sqrt{2} M_{P}$. Combining Equations (2.20) and (5.113) we get

$$
\beta^{2}(\phi)=\frac{3}{\mathcal{\kappa}}(1+w)
$$

In the $N$-formalism the Hubble parameter and hence the function $\beta(\phi)=\hat{\beta}(N)$ is given as a function of the number of e-folds, $N$. In this connection it is useful to note from Equations (3.7), (3.20) and (5.113) that

$$
\hat{\beta}^{2}(N)=\frac{\kappa}{2} \varepsilon_{H} \approx \frac{\kappa}{2} \varepsilon
$$

It follows from Equations (3.50) and (5.113) that the number of e-folds is

$$
N=\int_{\phi}^{\phi_{f}} \frac{d \phi}{\beta(\phi)}
$$

The inflaton field can be expressed as a function of the number of e-folds, as given in Equation (3.50), as

$$
\phi=\phi_{f}+\int_{N}^{0} \hat{\beta}(N) d N .
$$

In this formalism the value of the inflaton field at the end of the slow roll period is determined from the condition

$$
\beta\left(\phi_{f}\right)=1 \text {. }
$$

Let us consider an example. With Mukhanov's choice (5.82), Equation (5.116) gives (choosing the positive square root)

$$
\hat{\beta}(N)=\frac{\sqrt{3 \beta / \kappa}}{(N+1)^{\alpha / 2}},
$$

where $\beta$ without an argument is a constant. Inserting this into Equation (5.119) and performing the integration gives

$$
\phi(N)=\left\{\begin{array}{ll}
\phi_{0}-\frac{2}{2-\alpha} \sqrt{\frac{3 \beta}{\kappa}}(N+1)^{\frac{2-\alpha}{2}}, \quad \phi_{0}=\phi_{f}+\frac{2}{2-\alpha} \sqrt{\frac{3 \beta}{\kappa}}, \quad, \quad \alpha \neq 2 \\
\phi_{f}+\sqrt{\frac{3 \beta}{\kappa}} \ln (N+1) & , \quad \alpha=2
\end{array} .\right.
$$

Hence

$$
\beta(\phi)=\left\{\begin{array}{ll}
\left(\frac{\kappa}{3 \beta}\right)^{\frac{1}{\alpha-2}}\left[\frac{2-\alpha}{2}\left(\phi-\phi_{0}\right)\right]^{\frac{\alpha}{\alpha-2}}, \quad \alpha \neq 2 \\
\exp \left[-\sqrt{\frac{\kappa}{3 \beta}}\left(\phi-\phi_{f}\right)\right], \quad \alpha=2
\end{array} .\right.
$$


Inserting this into Equation (5.113) and integrating gives for $\alpha \neq 1,2$,

$$
H=\left\{\begin{array}{ll}
H_{0} \exp \left[-\frac{3 \beta}{2(\alpha-1)} \widetilde{\phi}^{2 \frac{\alpha-1}{\alpha-2}}\right], \widetilde{\phi} \equiv \sqrt{\frac{\kappa}{3 \beta} \frac{2-\alpha}{2}\left(\phi-\phi_{0}\right)}, \quad \alpha \neq 1,2 \\
H_{1}\left(\phi-\phi_{0}\right)^{3 \beta} & , \quad \alpha=1 \\
H_{f} \exp \left\{\sqrt{\frac{3 \beta}{\kappa}}\left[e^{-\sqrt{\frac{\kappa}{3 \beta}}\left(\phi-\phi_{f}\right)}-1\right]\right\} & , \quad \alpha=2
\end{array} .\right.
$$

Combining Equations (2.14) and (5.113) the potential of the inflaton field is given by

$$
\kappa V=H^{2}\left(3-\frac{\kappa}{2} \beta^{2}(\phi)\right) .
$$

Inserting the expressions (5.123) and (5.124) we get

$$
V(\phi)= \begin{cases}H_{0}^{2}\left[3-\frac{\kappa}{2}\left(\frac{\kappa}{3 \beta}\right)^{-2 \frac{\alpha-1}{\alpha-2}} \widetilde{\phi}^{\frac{2 \alpha}{\alpha-2}}\right] \exp \left[-\frac{3 \beta}{\alpha-1} \widetilde{\phi}^{\left.\frac{\alpha-1}{\alpha-2}\right]}\right. & , \quad \alpha \neq 1,2 \\ H_{1}^{2}\left(\phi-\phi_{0}\right)^{6 \beta}\left[3-\frac{9 \beta^{2}}{4 \kappa}\left(\phi-\phi_{0}\right)^{-2}\right] & , \quad \alpha=1 \\ {\left[3-\frac{\kappa}{2} \exp \left(-2 \sqrt{\frac{\kappa}{3 \beta}}\left(\phi-\phi_{f}\right)\right)\right] \exp \left\{2 \sqrt{\frac{3 \beta}{\kappa}} \exp \left[-\sqrt{\frac{\kappa}{3 \beta}}\left(\phi-\phi_{f}\right)\right]\right\},} & \alpha=2\end{cases}
$$

Differentiating Equation (5.113) and using the definitions (3.7) Binétruy et al. have calculated the Hubble slow roll parameters in terms of the $\beta$ - function and its derivatives with the result

$$
\varepsilon_{H}=\frac{1}{2} \beta^{2} \quad, \quad \eta_{H}=\frac{1}{2} \beta^{2}-\frac{\beta^{\prime}}{\kappa} \quad, \quad \xi_{H}=\frac{1}{4} \beta^{4}-\frac{\beta^{3 \prime}}{2 \kappa}+\frac{\beta \beta^{\prime \prime}}{\kappa^{2}} .
$$

Inserting these expressions into Equations (4.20), (4.29) and (4.44) the optical parameters may be expressed in terms of the $\beta$ - function and its derivatives as follows

$$
\begin{aligned}
& \delta_{n s}=\beta^{2}+\frac{2 \beta^{\prime}}{\kappa}, \quad r=8 \beta^{2}, \quad n_{t}=-\beta^{2}, \\
& \alpha_{s}=-\frac{2 \beta}{\kappa}\left(\beta \beta^{\prime}+\beta^{\prime \prime}\right) \quad, \quad \alpha_{t}=-\frac{2}{\kappa} \beta^{2} \beta^{\prime} .
\end{aligned}
$$

We see that $\beta^{2}$ is of the same order of magnitude as $\varepsilon_{H}$ which is usually of the order $10^{-2}$. Assuming that the derivatives of $\beta$ is of the same order of magnitude as $\beta$, it follows that $\varepsilon_{H}<<\eta_{H}$ and that we can use the approximations

$$
\kappa \eta_{H} \approx-\beta^{\prime}, \quad \kappa^{2} \xi_{H} \approx \beta \beta^{\prime \prime} .
$$

With these approximations we have

$$
\kappa \delta_{n s} \approx 2 \beta^{\prime}, \quad \kappa \alpha_{s} \approx-2 \beta \beta^{\prime \prime} .
$$

According to Equations (3.50) and (5.113) the relationship between derivatives with respect to $N$ and $\phi$ are

$$
\frac{d}{d N}=-\beta(\phi) \frac{d}{d \phi}
$$

Using this the Hubble slow roll parameters can be expressed as functions of $N$ as

$$
\varepsilon_{H}=\frac{1}{2} \hat{\beta}^{2} \quad, \quad \eta_{H}=\frac{1}{2} \hat{\beta}^{2}+(\ln |\hat{\beta}|)_{N}, \quad \xi_{H}=\frac{1}{4} \hat{\beta}^{4}-\frac{1}{2} \hat{\beta}^{2},_{N}+\frac{5}{2} \hat{\beta}^{2}(\ln |\hat{\beta}|)_{N}+(\ln |\hat{\beta}|)_{, N N} .
$$

To lowest order in $\hat{\beta}$ we have

$$
\eta_{H} \approx(\ln |\hat{\beta}|)_{N}, \quad \xi_{H} \approx(\ln |\hat{\beta}|){ }_{N N}=\eta_{H, N} .
$$


The optical parameters can be expressed as functions of $N$ as

$$
\delta_{n s} \approx \hat{\beta}^{2}-2(\ln |\hat{\beta}|)_{, N}, \quad r=8 \hat{\beta}^{2}, \quad n_{T}=-\hat{\beta}^{2}, \quad \alpha_{S} \approx \hat{\beta}^{2},_{N}-2(\ln |\hat{\beta}|)_{, N N}, \quad \alpha_{T} \approx \hat{\beta}^{2}{ }_{, N} .
$$

Using the approximations (5.130) we have

$$
\delta_{n s} \approx-2(\ln |\hat{\beta}|), N \quad, \quad \alpha_{S} \approx-2(\ln |\hat{\beta}|), N N .
$$

Note from Equation (5.132) that

$$
\alpha_{S}=\delta_{n s, N} .
$$

In order to give a classification of inflationary models Binétruy et al. now assume that the potential is close to an extremal point where the field has the value $\phi_{0}$, so that the $\beta$-function has the following expansion,

$$
\beta(\phi)=\beta_{1} \hat{\phi}^{q},
$$

where $\beta_{1}$ is a constant and $\hat{\phi}=\left(\phi-\phi_{0}\right) / M_{P}$. Inserting this into Equation (5.113) and integrating gives

$$
H=\left\{\begin{array}{ll}
H_{0} \exp \left[-\frac{\beta_{1} \sqrt{\kappa}}{2(1+q)} \hat{\phi}^{1+q}\right], & q \neq-1 \\
H_{1} \hat{\phi}^{-\kappa \beta_{1} / 2}, & q=-1
\end{array},\right.
$$

where $H_{0}=H\left(\phi_{0}\right)$. Inserting these expressions for $\beta$ and $H$ into Equation (5.125) gives

$$
V(\phi)=\left\{\begin{array}{ll}
H_{0}^{2}\left(3-\frac{\kappa \beta_{1}^{2}}{2} \hat{\phi}^{2 q}\right) \exp \left(-\frac{\beta_{1} \sqrt{\kappa}}{1+q} \hat{\phi}^{1+q}\right), & q \neq-1 \\
H_{1}^{2} \hat{\phi}^{-\kappa \beta_{1}}\left(3-\frac{\kappa}{2} \hat{\phi}^{-2}\right) & , \quad q=-1
\end{array} .\right.
$$

Sufficiently near the extremal point $\left[\beta_{1} \sqrt{\kappa} /(1+q)\right] \hat{\phi}^{1+q}<<1$ for $q>-1$. Binétruy et al. [82] have therefore used the approximation

$$
V(\phi) \approx H_{0}^{2}\left(3-\frac{\kappa \beta_{1}^{2}}{2} \hat{\phi}^{2 q}\right)\left(1-\frac{\beta_{1} \sqrt{\kappa}}{1+q} \hat{\phi}^{1+q}\right) .
$$

They made a classification with seven classes of inflationary models.

1. Monomial model. $q>1$. Then $2 q>1+q$ and we can make the further simplification

$$
V(\phi) \approx 3 H_{0}^{2}\left(1-\frac{\beta_{1} \sqrt{\kappa}}{1+q} \hat{\phi}^{1+q}\right) .
$$

Calculating the number of e-folds from Equation (5.118) then gives

$$
N=\frac{1}{\beta_{1}(q-1) \hat{\phi}^{q-1}}-N_{f} \quad, \quad N_{f}=\frac{1}{\beta_{1}(q-1) \hat{\phi}_{f}^{q-1}} .
$$

According to Equations (5.119) and (5.137) the value of the inflaton field at the end of the slow roll era is

$$
\hat{\phi}_{f}=\beta_{1}^{-1 / q},
$$

Giving

$$
N_{f}=\frac{1}{(q-1) \beta_{1}^{1 / q}} .
$$


Inserting this into Equation (5.142) gives

$$
\hat{\phi}(N)=\frac{1}{\beta_{1}^{1 / q}\left[(q-1) \beta_{1}^{1 / q} N+1\right]^{1 /(q-1)}} .
$$

From this equation together with Equation (5.129) we obtain

$$
\hat{\beta}(N)=\frac{1}{\left[(q-1) \beta_{1}^{1 / q} N+1\right]^{q /(q-1)}} .
$$

Assuming that $(q-1) \beta_{1}^{1 / q} N>>1$ this can be approximated by

$$
\hat{\beta}(N) \approx \frac{1}{\beta_{1}^{1 /(q-1)}[(q-1) N]^{q /(q-1)}} .
$$

Using this expression in Equation (5.134) we obtain for $\delta_{n s}, r$ and $\alpha_{S}$

$$
\delta_{n s} \approx \frac{2 q}{q-1} \frac{1}{N} \quad, \quad r \approx \frac{8}{\beta_{1}^{2 /(q-1)}[(q-1) N]^{2 q /(q-1)}} \quad, \quad \alpha_{S} \approx-\frac{2 q}{q-1} \frac{1}{N^{2}} .
$$

Hence

$$
r \approx 8\left(\frac{\delta_{n s}}{2 \beta_{1} q}\right)^{2 q /(q-1)}, \quad \alpha_{S} \approx-\frac{q-1}{2 q} \delta_{n s}^{2}
$$

The function $r(q)$ as given in Equation (5.147) is plotted in Figure 3 for $4<q<12$.

We see that $r<5.5 \times 10^{-6}$. Hence the monomial class of models predicts a practically speaking vanishing tensor-to-scalar ratio. Furthermore $\alpha_{S}$ decreases from zero to $-(1 / 2) \delta_{n s}^{2}$ when $q$ increases from 1 to infinity.

2. The linear class. $q=1$. From Equations (5.138) and (5.139) we then have

$$
\begin{gathered}
\beta(\phi)=\beta_{1} \hat{\phi}, \\
H(\phi)=H_{0} \exp \left(-\frac{\beta_{1} \sqrt{\kappa}}{4} \hat{\phi}^{2}\right), \\
V(\phi) \approx 3 H_{0}^{2}\left(1-\frac{\beta_{1} \sqrt{\kappa}}{2} \hat{\phi}^{2}\right) .
\end{gathered}
$$

Using Equation (5.118) the number of e-folds is

$$
N=-\frac{1}{\beta_{1}} \ln \left(\beta_{1} \hat{\phi}\right)
$$

Hence

$$
\hat{\beta}(N)=e^{-\beta_{1} N}
$$

Note that with the expression (5.154) it follows that $(\ln \hat{\beta})_{, N N}=0$, so in this case we need the accurate expressions (5.133). This gives the optical parameters

$$
\delta_{n s}=2 \beta_{1}+e^{-2 \beta_{1} N} \quad, \quad r=8 e^{-2 \beta_{1} N} \quad, \quad \alpha_{S}=-2 \beta_{1} e^{-2 \beta_{1} N} .
$$


If $e^{-2 \beta_{1} N}<<\beta_{1}$ then

$$
\delta_{n s} \approx 2 \beta_{1},
$$

and we have the consistency relationships

$$
r=8 e^{-N \delta_{n s}}, \quad \alpha_{S}=-\delta_{n s} e^{-N \delta_{n s}} .
$$

Inserting $N=60, \delta_{n s}=0.032$ gives $r=1.18$ which is much too large compared with the BPK-observations.

3. Inverse field monomial class. $\phi_{0}=0, q<-p, p>1$. This is an example of large field inflation. The leading term of the $\beta$ - function is now

$$
\beta(\phi) \simeq-\frac{\beta_{2}}{\hat{\phi}^{p}}
$$

In this case the $r, \delta_{n s}-$ relationship takes the form

$$
r \approx 8\left(\frac{\delta_{n s}}{2 \beta_{1} p}\right)^{2 p /(p+1)}
$$

which is plotted as a function of $\mathrm{p}$ in Figure 4. We see that $r<0.04$ requires $p>1.5$.

Furthermore

$$
\alpha_{S} \approx-\frac{p+1}{2 p} \delta_{n S}^{2}
$$

Hence $\alpha_{S}$ increases from $-\delta_{n s}^{2}$ to $-(1 / 2) \delta_{n s}^{2}$ when $p$ increases from 1 to infinity.

4. Chaotic class, $p=1$. Then

$$
\beta(\phi) \simeq-\frac{\beta_{2}}{\hat{\phi}}
$$

where $0<\beta_{2}<1$. In this case integration of Equation (5.113) gives the Hubble parameter

$$
H(\phi)=H_{1} \hat{\phi}^{\kappa \beta_{2} / 2} .
$$

From Equation (5.125) we then find the potential

$$
\kappa V(\phi)=H_{1}^{2} \hat{\phi}^{\kappa \beta_{2}}\left(3-\frac{\kappa \beta_{2}^{2}}{2 \hat{\phi}^{2}}\right) .
$$

In the large field case $\kappa \beta_{2}^{2} / 2 \hat{\phi}^{2}<<1$, and the potential can be approximated by

$$
V(\phi) \approx 3 H_{1}^{2} \hat{\phi}^{\kappa \beta_{2}} .
$$

Using Equation (5.118) the number of e-folds is

$$
N=\frac{1}{2 \beta_{2}}\left(\hat{\phi}^{2}-\hat{\phi}_{f}^{2}\right) .
$$

Combining this equation with Equation (5.161) gives

$$
\hat{\beta}(N)=-\frac{\beta_{2}}{\sqrt{\phi_{f}^{2}+2 \beta_{2} N}} .
$$


The slow roll era ends when $\beta\left(\phi_{f}\right)=1$ giving $\phi_{f}^{2}=\beta_{2}^{2}$. Hence

$$
\hat{\beta}(N)=-\left(1+\frac{2}{\beta_{2}} N\right)^{-1 / 2}
$$

With $\beta_{2}<1$ we have $2 N / \beta_{2}>>1$, so we can approximate $\hat{\beta}$ by

$$
\hat{\beta}_{2}(N) \approx \sqrt{\beta_{2} / 2 N}
$$

Using this in Equation (5.134) gives the optical parameters

$$
\delta_{n S} \approx \frac{1+\beta_{2} / 2}{N}, \quad r \approx \frac{4 \beta_{2}}{N}, \quad \alpha_{S} \approx-\frac{1+\beta_{2} / 2}{N^{2}}
$$

It follows from these relationships that

$$
\beta_{2}=2\left(N \delta_{n s}-1\right)
$$

and

$$
r=8\left(\delta_{n s}-1 / N\right) \quad, \quad \alpha_{S}=-\delta_{n s}\left(\delta_{n s}-\frac{r}{8}\right)=-\frac{\delta_{n s}}{N} .
$$

Inserting $N=60, \delta_{n s}=0.032$ gives $\beta_{2}=1.8, r=0.13$ and $\alpha_{S}=-0.0005$. The value of the tensor-to-scalar ratio is too large to be compatible with the BPK-observations.

5. Fractional class, $0<p<1$. For this class of inflationary models

$$
r=8 \delta_{n s}=\frac{\beta_{2}^{2 /(p+1)}}{[(p+1) N]^{2 p /(p+1)}} \quad, \quad \alpha_{S}=-\frac{2 p \beta_{2}^{2 /(p+1)}}{[(p+1) N]^{(3 p+1) /(p+1)}} .
$$

Hence $\delta_{n s}=0.032$ gives $r=0.256$. This prediction is in conflict with the BPK-data.

6. Power law class, $p \rightarrow 0$. In this limit the $\beta$ - function is constant, $\beta(\phi)=\beta_{2}$. Integration of

Equation (5.113) then gives the Hubble parameter as a function of the inflaton field

$$
H=H_{2} e^{\left(\kappa \beta_{2} / 2\right) \phi} .
$$

Equation (5.125) then gives the potential

$$
V(\phi)=\left(3-\frac{\kappa}{2} \beta_{2}^{2}\right) e^{\kappa \beta_{2} \phi}
$$

Integration of Equation (5.114) gives the Hubble parameter as a function of time

$$
H=\frac{1}{H_{0}+\left(\kappa \beta_{2}^{2} / 2\right) t} \text {. }
$$

Letting $H_{0}=0$ we have a Big Bang with infinitely great Hubble parameter at the initial moment, $H(0)=\infty$. In this case the scale factor is

$$
a(t)=a_{1}\left(t / t_{1}\right)^{2 / \kappa \beta_{2}^{2}} .
$$


Hence there is power law expansion, which is the reason for the name "power law class" of this case. For this class of inflationary models the optical parameters are

$$
\delta_{n s}=\frac{\beta_{2}^{2}}{1-\beta_{2}^{2} / 2} \quad, \quad r=8 \beta_{2}^{2} \quad, \quad \alpha_{S}=0,
$$

giving

$$
r=\frac{8 \delta_{n s}}{1+\delta_{n s}} \approx 8 \delta_{n s} .
$$

Like the previous class of inflationary models those of this class are ruled out by the BPK-data.

7. Exponential class

$$
\beta(\phi)=-\beta_{2} \exp (-\gamma \hat{\phi}),
$$

where $\gamma$ is a positive constant. This has the same form as the $\beta$ - function of Equation (5.122) for $\alpha=2$. In this case the optical parameters are

$$
\delta_{n s} \approx \frac{2}{N} \quad, \quad r \approx \frac{8}{\gamma^{2} N^{2}} \quad, \quad \alpha_{S}=-\frac{2}{N^{2}} .
$$

For this class of models

$$
r=\left(2 / \gamma^{2}\right) \delta_{n s} \quad, \quad \alpha_{S}=-(1 / 2) \delta_{n s}^{2} .
$$

The BPK-data, $\delta_{n s}=0.032, r<0.04$ requires $\gamma>1.3$.

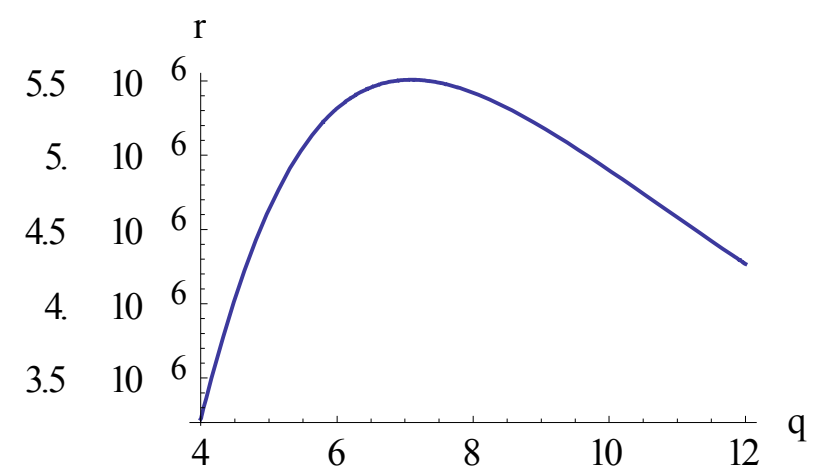

Figure 3. The tensor-to-scalar ratio plotted as function of $q$ for $\beta=1$ and $\delta_{n s}=0.032$.

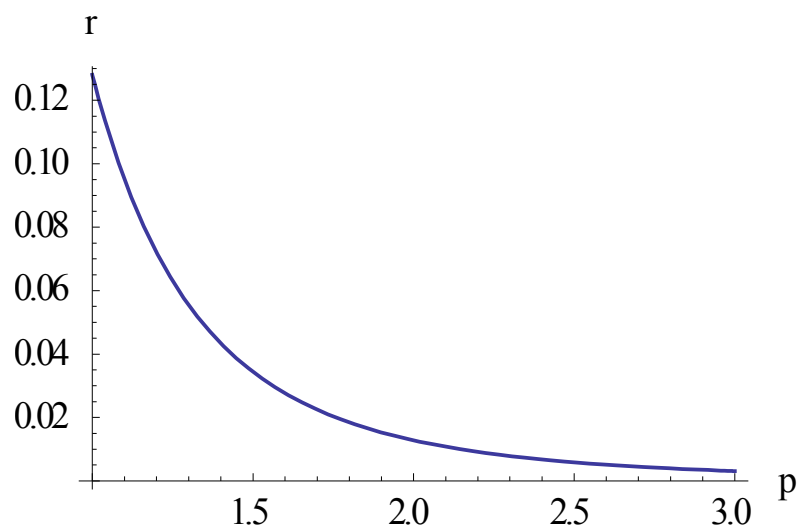

Figure 4. The tensor-to-scalar ratio plotted as function of $p$ for $\beta=1$ and $\delta_{n s}=0.032$. 


\section{Predictions from Different Inflationary Models}

Different inflation models have different physical motivations. However the calculations of the predictions of the models for observable quantities are usually calculated in the same way using the slow roll formalism. One often calculates numerically $n_{S}$ versus $r$ diagrams (see for example Okada et al., 2014). Then the differences between the models cook down to specifying the potential by different functions of the potential, $V(\phi)$. In this connection one often classifies the models in three classes:

Large field inflation: In these models the field strength at the end of the inflation is larger than an order of magnitude less than the Planck energy. Small field inflation: Models where the field strength at the end of the inflation is several orders of magnitude less than the Planck energy. Hybrid inflation: This is a class of models where the inflationary era ends due to an extra field different from the inflaton field that dominates during the slow roll era.

For the models that are considered in the present article we shall calculate all or some of the expressions for the spectral parameters $n_{S}, n_{T}, r, \alpha_{S}, \alpha_{T}$ that may be used to judge how successful the models are in relation to the Planck- and BICEP2 data and future data coming the next years. Particular focus will be put on the $\delta_{n s}, r$ - relationship which is well constrained by the latest observations.

There has not yet been any general agreement as to how the different inflation models should be named. I suggest that the models are given names after the mathematical form of the potential as a function of the inflaton field.

\subsection{Polynomial Chaotic Inflation}

The so-called chaotic inflation [83] was proposed by A. Linde [84], and is a class of polynomial inflation models. They are large field inflation models. The potential of the inflaton field in this type of inflationary models is of the large field inflation type and has a potential (Martin et al. [27] and Clesse [85],

$$
V=M^{4} \hat{\phi}^{p}
$$

where $\hat{\phi}=\phi / M_{P}$, and $M$ is the energy scale of the potential when the inflaton field has Planck mass. It is assumed that $p$ is constant and that $\phi>0$. The class of models with $n=-p$ and $n>0$ is called the inverse power-law inflation and has been investigated by J. D. Barrow and A. R. Liddle [86] and by K. Rezazadeh, K. Karami and S. Hashemi [87] in the context of tachyon inflation.

For this potential Equation (2.17) leads to

$$
\dot{\hat{\phi}}=A \hat{\phi}^{\frac{p}{2}-1}, \quad A=-\frac{p M^{2}}{\sqrt{3}} .
$$

The solution of this equation with the inflaton field equal to the Planck mass at the Planck time gives the time evolution of the inflaton field during the slow roll era.

$$
\phi(t)=M_{P}\left(1+\frac{4-p}{2 M_{P}} A\left(t-t_{P}\right)\right)^{\frac{2}{4-p}} \quad, \quad p \neq 4
$$

Hence, the inflaton field depends linearly upon time for $p=2$. Inserting this into Equation (6.1.1) gives the time evolution of the potential,

$$
V(t)=M^{4}\left(1-\frac{4-p}{2 M_{P}} A\left(t-t_{P}\right)\right)^{\frac{2 p}{4-p}} \quad, \quad p \neq 4 .
$$

For $p=4$ the time evolution of the inflaton field and the potential is

$$
\phi(t)=M_{P} e^{\left(A / M_{P}\right)\left(t-t_{P}\right)}
$$


and

$$
V(t)=M^{4} e^{\left(2 A / M_{P}\right)\left(t-t_{P}\right)} .
$$

From Equation (2.16) it then follows that the time evolution of the Hubble parameter is

$$
H(t)=\left\{\begin{array}{ll}
\frac{1}{\sqrt{3}} \frac{M^{2}}{M_{P}}\left[1+\frac{4-p}{2 M_{P}} A\left(t-t_{P}\right)\right]^{\frac{p}{4-p}}, & p \neq 4 \\
\frac{1}{\sqrt{3}} \frac{M^{2}}{M_{P}} e^{\left(A / M_{P}\right)\left(t-t_{P}\right)} & , \quad p=4
\end{array} .\right.
$$

We shall now deduce expressions for the spectroscopic parameters of this model in terms of the number of e-folds. Differentiating the potential we get

$$
\frac{V^{\prime}}{V}=\frac{p}{\hat{\phi}}, \quad \frac{V^{\prime \prime}}{V}=\frac{p(p-1)}{\hat{\phi}^{2}}, \quad \frac{V^{\prime \prime \prime}}{V}=\frac{p(p-1)(p-2)}{\hat{\phi}^{3}} .
$$

The potential slow roll parameters for this model are

$$
\varepsilon=\frac{1}{2}\left(\frac{p}{\hat{\phi}}\right)^{2}, \quad \eta=\frac{p(p-1)}{\hat{\phi}^{2}}, \quad \xi=\frac{p^{2}(p-1)(p-2)}{\hat{\phi}^{4}},
$$

For this class of inflationary universe models we therefore have

$$
\eta=\frac{2(p-1)}{p} \varepsilon, \quad \xi=\frac{4(p-1)(p-2)}{p^{2}} \varepsilon^{2},
$$

Note that $\eta=\xi=0$ for $p=1$. From Equation (3.22) we then get

$$
\eta_{H}=-\varepsilon_{H}
$$

which has been called the linear model by Kinney et al. [88]. The definition (3.7) gives in this case the equation

with the general solution

$$
\frac{H^{\prime \prime}}{H^{\prime}}+\frac{H^{\prime}}{H}=0
$$

$$
H(\phi)=\left(C_{1} \phi+C_{2}\right)^{1 / 2},
$$

where $C_{1}$ and $C_{2}$ are constants of integration. Using Equation (2.16), we then obtain the linear relationship

$$
V(\phi)=\left(3 M_{P}^{2}\right)\left(C_{1} \phi+C_{2}\right) .
$$

Equation (3.12), on the other hand, gives in this case

$$
\frac{\ddot{H}}{\dot{H}}+2 \frac{\dot{H}}{H}=0
$$

with the solution

$$
H(t)=\left(C_{3} t+C_{4}\right)^{1 / 3} .
$$

Inserting the expressions (6.1.9) into Equations (4.4), (4.13), and (4.29) we get

$$
\delta_{n s}=\frac{p(p+2)}{\hat{\phi}^{2}}, \quad r=\frac{8 p^{2}}{\hat{\phi}^{2}} \quad, \quad \alpha_{S}=-\frac{2 p^{2}(p+2)}{\hat{\phi}^{4}} .
$$


For this model there is also a simple expression for $\beta_{S}$ as given in Equation (4.32) (Amorós and Haro [56])

$$
\beta_{S}=-\frac{8 p^{3}(p+2)}{\hat{\phi}^{6}} \text {. }
$$

When comparing with the first of the expressions (6.1.17) we obtain

$$
\beta_{S}=-\frac{8}{(p+2)^{2}} \delta_{n S}^{3}
$$

Equation (6.1.17) gives the $\delta_{n s}, r-$ and $r, \alpha_{S}-$ relations

$$
r=\frac{8 p}{p+2} \delta_{n s} \quad, \quad \alpha_{S}=-\frac{2}{p+2} \delta_{n s}^{2}=-\frac{1}{32} \frac{p+2}{p^{2}} r^{2}=\left(\frac{r}{8}-\delta_{n s}\right) \delta_{n s} \quad, \quad p=\frac{2 r}{8 \delta_{n s}-r} .
$$

With $\delta_{n s}=0.032$ and for example $p=2$ we get $r=0.128, \alpha_{S}=-0.0005$ and $\beta_{n s}=-0.000016$. This value of $r$ is larger than admitted by the by the BPK-data. Also these data prefer larger absolute values of $\alpha_{S}$ and $\beta_{S}$. However smaller values of $p$ may give acceptable values of $r$. Inserting for example $r=0.05, \delta_{n s}=0.032$ in the expression (6.1.20) for $p$ gives $p=0.64$. Ballesteros and Casas [31] conclude that the single field inflationary model with a quadratic potential predicts too little running of the spectral index.

It follows from Equations (4.54) and (6.1.20) that for this class of inflationary models the running of the tensor-to-scalar ratio is

$$
\hat{\alpha}_{r}=\frac{2}{p+2} \delta_{n s} .
$$

Hence

$$
p=2\left(\frac{\delta_{n s}}{\hat{\alpha}_{r}}-1\right)
$$

For inflationary models with the standard Bunch-Davis initial conditions for cosmic perturbations the B2014-Planck results required $\hat{\alpha}_{r} \geq 0.16$ which gives $p \leq-1.6$, corresponding to inverse power law inflation.

One may also try to determine the value of $p$ from observable data. Solving the expressions (6.1.20) with respect to $p$ we get

$$
p=\frac{2 r}{8 \delta_{n s}-r}=-\frac{2 \delta_{n s}^{2}}{\alpha_{S}}-2=-\frac{r \delta_{n s}}{4 \alpha_{S}} .
$$

Due to the large uncertainties in the measured value of $r$ and $\alpha_{S}$ one may obtain different values of $p$ from these expressions. With the Planck/BICEP2 2015 center values $\delta_{n s}=0.032, r=0.05$ the first expression gives $p \approx 0.5$.

Note for $p=2$ one obtains $\varepsilon=\eta=2 / \hat{\phi}^{2}$, and the slow roll condition $\varepsilon, \eta<<1$ takes the form $\hat{\phi}>>\sqrt{2}$, i.e., the inflaton field is larger than the Planck energy, making this inflationary model rather speculative as long as we lack a generally accepted quantum gravity theory.

Equation (6.1.9) gives the consistency relation (Chiba and Kohri [89])

$$
\xi=2 \eta(\eta-\varepsilon) .
$$

Inserting the expressions (3.1) for the slow roll parameters one obtains the differential equation

$$
V V^{\prime} V^{\prime \prime \prime}+V^{\prime 2} V^{\prime \prime}-2 V V^{\prime \prime 2}=0 .
$$

The general solution of this equation is

$$
V(\phi)=\left(K_{2} \phi+K_{3}\right)^{K_{1}}
$$


where $K_{1}, K_{2}, K_{3}$ are integrations constants. Hence the consistency relation (6.1.24) is valid for all inflationary mono-field models with potential of this form.

It follows from the expressions (6.1.17) that

$$
\alpha_{S}=\varepsilon^{2}-(5 \varepsilon-2 \eta)^{2} .
$$

Hence for this class of universe models $\alpha_{S}<\varepsilon^{2}$. Using Equation (4.24), Equation (6.1.27) may be written as a relation between observables

$$
\alpha_{S}=-\delta_{n s}\left(\delta_{n s}-r / 8\right),
$$

or

$$
r=\left(8 / \delta_{n s}\right)\left(\delta_{n s}^{2}+\alpha_{S}\right) .
$$

Together with the Planck data this formula shows that there is a problem with the polynomial inflation model. The ratio $r$ must be positive, which requires $\alpha_{S}>-\delta_{n s}{ }^{2}$. However the best fit Planck data are $\delta_{n s}=0.032$, giving $\delta_{n s}{ }^{2}=0.001$, and $\alpha_{S}=-0.003$, which is less than $-\delta_{n s}{ }^{2}$. Hence the Planck data and Equation (6.1.29) gives a negative value of r. As noted by Ade et al. [36] this class of inflationary models is now ruled out by the observational data.

It follows from Equations (3.43) and (6.1.28) that the ratio of the running of the spectral index of tensor and scalar fluctuations is

$$
\frac{\alpha_{T}}{\alpha_{S}}=\frac{r}{8 \delta_{n s}} .
$$

for the polynomial inflationary models.

It is usual to define the end of the inflationary era by $\varepsilon\left(\phi_{f}\right)=1$. Using that $\kappa=1 / M_{P}^{2}$, it then follows that the value of the inflaton field at the end of the slow roll era is (Remmen and Carroll, 1985)

$$
\phi_{f}=(p / \sqrt{2}) M_{P} .
$$

From the first of Equation (4.1.9) we have

$$
\phi=\frac{p M_{P}}{\sqrt{2 \varepsilon}} .
$$

Hence $\phi / \phi_{f}=1 / \sqrt{\varepsilon}$, and during the slow roll era with $\varepsilon, \eta<<1$ the inflaton field is $\phi>>\phi_{f}$ and $\phi>>M_{P}$.

From Equations (3.50) and (6.1.9) we have

$$
d N=\frac{\kappa}{p} \phi d \phi
$$

Integrating through the slow roll inflationary era, we get

$$
N=\frac{\kappa}{2 p}\left(\phi^{2}-\phi_{f}^{2}\right)
$$

where $\phi=\phi(N)$ is the value of the field strength when the slow roll era with $N$ e-folds begins. Inserting the expression (6.1.25) for $\phi_{f}$ into Equation (6.1.27) it follows that the initial value of the field strength is

$$
\phi=\sqrt{\frac{p}{2}(p+4 N)} M_{P}
$$


It then follows from Equations (6.1.9) and (6.1.34) that for an inflationary era in which the potential of the dark energy is a power of the scalar field, the slow roll parameters are

$$
\varepsilon=\frac{p}{p+4 N} \quad, \quad \eta=\frac{2(p-1)}{p+4 N} \quad, \quad \xi=\frac{4(p-1)(p-2)}{(p+4 N)^{2}} .
$$

From Equations (4.13), (4.15), (4.4), (4.29) and (4.43) respectively, we then get

$$
\delta_{n s}=\frac{2(p+2)}{p+4 N}, \quad n_{T}=-\frac{2 p}{p+4 N}, \quad r=\frac{16 p}{p+4 N}, \quad \alpha_{S}=-\frac{8(p+2)}{(p+4 N)^{2}}, \quad \alpha_{T}=-\frac{8 p}{(p+4 N)^{2}} \text {. }
$$

Hence

$$
\frac{r}{\delta_{n s}}=\frac{8 p}{p+2} \quad, \quad-\frac{n_{T}}{\delta_{n s}}=\frac{\alpha_{T}}{\alpha_{S}}=\frac{p}{p+2} .
$$

There is an arbitrariness in this inflationary model, because there is no mechanism determining when the inflationary era starts or ends. A start with field strength much larger than the Planck energy such as in Equation (6.1.32), does not seem very natural in the domain of a non-quantum theory. Hence, there has been proposed that the chaotic inflation period starts at the Planck time with $V\left(\phi_{1}\right)=M_{P}^{4}$ and ends with $\phi_{f}<<\phi$ so that $\phi_{f}$ can be neglected in Equation (6.1.34). Equation (6.1.35) then reduces to

$$
\phi=(2 p N)^{1 / 2} M_{P}
$$

Equation (6.1.1) with $V(\phi)=M_{P}^{4}$ together with Equation (6.1.37) gives

$$
M=M_{P} /(2 p N)^{p / 8} \text {. }
$$

The most simple chaotic inflationary universe model has $p=2$. Typically one requires about 50-fold increase of the scale factor during the slow roll era in order to solve the monopole, horizon and flatness problems, giving $M=0.26 M_{P}$.

Calculating the spectroscopic parameters, one finds that putting $\phi_{f}=0$ corresponds to neglecting $p$ in the denominator in the expressions (6.1.36). Usual values for $p$ and $N$ are 2 and 50. With these values the slow roll parameters and the spectroscopic parameters will be changed only by about one per cent if $p$ is neglected in the denominator. Therefore, the arbitrariness of the final point of time of the slow roll era in this model does not influence the predicted values of the spectroscopic parameters seriously. The expressions are then simplified to

$$
\varepsilon \approx \frac{p}{4 N}, \quad \eta \approx \frac{p-1}{2 N}, \quad \xi \approx \frac{(p-1)(p-2)}{4 N^{2}}
$$

and

$$
\delta_{n s}=\frac{p+2}{2 N}, \quad n_{T}=-\frac{p}{2 N} \quad, \quad r=\frac{4 p}{N}, \quad \alpha_{S}=-\frac{p+2}{2 N^{2}}, \quad \alpha_{T}=-\frac{p}{2 N^{2}} .
$$

The values of these quantities for $N=50$ and different values of $p$ are shown in Table 1 .

The values of $n_{S}$ predicted by polynomial inflation are roughly in agreement with the value from the Planck data, $n_{S}=0.968$ but the predicted value of $\alpha_{S}$ is more than ten times smaller than the value $\alpha_{S}=-0.003$ from the Planck data except for large values of $p$. Also, the quartic potential inflation model and models with higher powers of the inflaton field, are in conflict within observational data with too small predicted values of $n_{S}$ and too large values of $r$. The value of $p$ can be determined by

$$
p=\frac{4\left(N \delta_{n s}-1\right)}{1+n_{S}} \approx 2\left(N \delta_{n s}-1\right),
$$


where the first expression is calculated from Equation (6.1.37) and the second from Equation (6.1.42). For the Planck data in combination with $N=50$ this gives $p=1.2$ leading to a value of $\alpha_{S}$ in conflict with the Planck data. Similarly $r$ can be expressed in terms of $N$ and $\delta_{n s}$ by

$$
r=\frac{8 p}{p+2} \delta_{n s}=\frac{16\left(N \delta_{n s}-1\right)}{2 N-1} \approx 8\left(\delta_{n s}-\frac{1}{N}\right)
$$

giving $r=0.10$ which is larger than the value favored by the BPK-analysis (Ade et al., $2015 \mathrm{~A}$ ).

The equations above are accurate to first order in $\varepsilon$. Creminelli et al. [12] have considered more accurate relationships valid to second order in $\varepsilon$ for the case of a quadratic potential. With $p=2$ the expressions (6.1.37) gives $\eta=\varepsilon, \xi=0$. Then Equations (4.18) and (4.19) give

$$
\delta_{n s}=4 \varepsilon+\frac{2}{3}(7+12 C) \varepsilon^{2} \quad, \quad r=16 \varepsilon-\frac{32}{3}(1-3 C) \varepsilon^{2} .
$$

From these equations we get

$$
\delta_{n s}-r / 4=(22 / 3) \varepsilon^{2}
$$

Hence the quantity $\delta_{n s}-r / 4$ is small to second order in $\varepsilon$. In order to obtain an $n_{S}, r$-relation accurate to second order in $\varepsilon$ we can therefore substitute for $\varepsilon$ from the first order approximations of Equation (6.1.45) where we neglect the second order terms, i.e., $\varepsilon \approx(1 / 4) \delta_{n s}$ and $\varepsilon \approx(1 / 16) r$. This gives the alternative relationships

$$
\delta_{n s}-r / 4=(11 / 24) \delta_{n s}^{2}=(11 / 384) r^{2} .
$$

The corresponding expressions with an arbitrary value of $p$ are

$$
\delta_{s n}=\frac{2(p+2)}{p} \varepsilon+\frac{8(p+2) C}{p^{2}} \varepsilon^{2}+\frac{2\left(3 p^{2}+14 p-12\right)}{3 p^{2}} \varepsilon^{2}, \quad r=16 \varepsilon-\frac{64(1-3 C)}{3 p} \varepsilon^{2},
$$

and

$$
\delta_{n s}-\frac{p+2}{8 p} r=\frac{2\left(3 p^{2}+18 p-4\right)}{3 p^{2}} \varepsilon^{2}
$$

giving

$$
\delta_{n s}-\frac{p+2}{8 p} r=\frac{3 p^{2}+18 p-4}{6(p+2)^{2}} \delta_{n s}^{2}=\frac{3 p^{2}+18 p-4}{384 p^{2}} r^{2} .
$$

Kobayashi and Seto [90] and Martin et al. [33] have considered inflationary models with potentials

$$
V(\phi)=(1 / 2) m^{2} \hat{\phi}^{2}+(1 / 4) \sigma \hat{\phi}^{4},
$$

and

$$
V=V_{0}-(1 / 2) m^{2} \hat{\phi}^{2}+(1 / 4) \sigma \hat{\phi}^{4} .
$$

They showed that with suitable values of $m$ and $\lambda$ such models predict values of the spectroscopic parameters in accordance with observations.

Trinomial inflation with

$$
V(\phi)=\frac{m^{2}}{2} \hat{\phi}^{2}+\frac{m g}{3} \hat{\phi}^{3}+\frac{\sigma}{4} \hat{\phi}^{4}
$$

has been thoroughly discussed by Destri et al. [91] and Martin et al. [33].

J. de Haro, J. Amorós and S. Pan [92] have studied inflationary models with the potential

$$
V(\phi)=3\left(H_{E} M_{P}\right)^{2}\left(\frac{\phi}{\phi_{E}}\right)^{\frac{2 \alpha}{2-\alpha}}\left[\left(\frac{\phi}{\phi_{E}}\right)^{2}-1\right], \quad \phi<\phi_{E}, \quad V(\phi)=0, \quad \phi>\phi_{E},
$$


where $H_{E}=H(0)$ is a fixed value of the Hubble parameter at the chosen origin of time, $\phi_{E}{ }^{2}=8 M_{P}^{2} / 3(2-\alpha)^{2}$, and we have put the cosmological constant equal to zero in their expressions. They have deduced this form of the potential from the assumption that the rate of change of the Hubble parameter with time is given by

$$
\dot{H}=-3 H_{E}^{2-\alpha} H^{\alpha} \quad, \quad H>H_{E} .
$$

Hence the Hubble slow roll parameters $\varepsilon_{H}$ and $\eta_{H}$ given in Equation (3.12) are

$$
\varepsilon_{H}=3\left(\frac{H_{*}}{H_{E}}\right)^{\alpha-2}, \quad \eta_{H}=\frac{\alpha}{2} \varepsilon_{H}
$$

where $H_{*}$ is the value of the Hubble parameter at the initial moment of the slow roll era. Inserting the expressions (6.1.55) and (6.1.56) into Equation (3.12) and then using Equations (4.20) and (4.29), the spectral index parameter $\delta_{n s}$ its running $\alpha_{s}$ and the tensor-to-scalar ratio $r$ are

$$
\delta_{n s}=(4-\alpha) \varepsilon_{H} \quad, \quad \alpha_{s}=(\alpha-2)(4-\alpha) \varepsilon_{H}^{2} \quad, \quad r=16 \varepsilon_{H} .
$$

Hence, we have the relationships

$$
r=\frac{16}{4-\alpha} \delta_{n s} \quad, \quad \alpha_{s}=\frac{\alpha-2}{4-\alpha} \delta_{n s}^{2} .
$$

For these models $\dot{H}$ is given in Equation (6.1.55) as a function of $H$. Then it will be advantageous to write Equation (3.62) for the number of e-folds as

$$
N=\int_{H_{*}}^{H_{\text {end }}} \frac{H}{\dot{H}} d H .
$$

where $H_{\text {end }}$ is the value of $H$ at the end of the slow roll era. Inserting the expression (6.1.55) for $\dot{H}$ and performing the integration gives

$$
N=\frac{1}{2-\alpha}\left(\frac{1}{\varepsilon_{*}}-\frac{1}{3}\right) \quad, \quad \varepsilon_{*}=3\left(\frac{H_{*}}{H_{E}}\right)^{\alpha-2}
$$

with $H_{\text {end }}=H_{E}$. Solving this equation with respect to $\varepsilon_{*}$ gives

$$
\varepsilon_{*}=\frac{1}{1 / 3+(2-\alpha) N} .
$$

Inserting this into Equation (6.1.57) gives

$$
\delta_{n s}=\frac{4-\alpha}{1 / 3+(2-\alpha) N} \quad, \quad r=\frac{16 \alpha}{1 / 3+(2-\alpha) N} \quad, \quad \alpha_{s}=\frac{(\alpha-2)(4-\alpha)}{[1 / 3+(2-\alpha) N]^{2}} .
$$

The first of the Equations (6.1.58) and (6.1.62) may be written, respectively, as

$$
\alpha=4\left(1-4 \frac{\delta_{n s}}{r}\right)
$$

and

$$
\alpha=2 \frac{N \delta_{n s}-2}{N \delta_{n s}-1} .
$$


Compatibility of these equations requires

$$
r=8\left(\delta_{n s}-1 / N\right)
$$

or

$$
N=\frac{1}{\delta_{n s}-r / 8}
$$

J. de Haro, J. Amorós and S. Pan [92] required $0 \leq \alpha \leq 1$. It is usual to require that the inflationary last for between 50 and 60 e-folds in order to solve the horizon- and flatness problems adequately. In order to make some estimates we will therefore assume that $50<N<60$. Also we will use the Planck value of the scalar optical parameter, i.e., $\delta_{n s}=0.032$. With these values Equation (6.1.65) gives $0.010<r<0.012$ which is allowed by the BPK-observations. Equations (5.1.63) and (5.1.64) then give respectively $-1.12<\alpha<-0.27$ and $-1.33<\alpha<-0.17$. Hence positive values of $\alpha$ are not allowed by the observational data.

\subsection{Hilltop Inflation}

Think of the curve describing the potential as a function of a scalar field. The name hilltop inflation (Boubekeur and Lyth [93]) refers to the case that inflation occurs near a local maximum of this curve, i.e., near a point where $V^{\prime}(\phi)=0$. With local symmetry around this point, having $V(0)=M^{4}$, the most simple version of the potential is written (Kohri, Lin and Lyth [94])

$$
V(\phi) \approx M^{4}\left(1-\frac{1}{2} \eta_{0} \hat{\phi}^{2}\right), \quad \eta_{0}>0
$$

where $\eta_{0}$ is a positive constant.

Inflationary models with this potential have also been called "New inflation". Here $\eta_{0}$ is the absolute value of the slow roll parameter $\eta$ at the maximum of the potential. Hill-top inflation occurs for small $\eta_{0}$.

Hilltop inflationary models may appear as the result of considering models where the potential is a more complicated function of the inflaton field and then making a series expansion. S. Basilakos et al. [95], for example, have considered a Starobinsky-like inflationary scenario with potential

$$
V(\phi)=M^{4} \frac{1+(1 / 3) \sinh ^{2}(n \hat{\phi} / 2)}{\cosh ^{2(n+2) / n}(n \hat{\phi} / 2)} .
$$

Assuming that $n \phi<<1$ and performing a Taylor expansion to second order in $n \phi$ one obtains

$$
V(\phi) \approx M^{4}\left[1-\frac{n(n+3)}{6} \hat{\phi}^{2}\right] .
$$

This potential has the Hilltop form (6.2.1) with

$$
\eta_{0}=(1 / 3) n(n+3) .
$$

Also it is sometimes assumed that

$$
\hat{\phi}=\phi / M_{P}<<1,
$$

which may not be a good approximation in light of the Lyth relationship (4.7). With this assumption it is usual to calculate the slow roll parameters with the approximation $V \approx M^{4}$ in the numerator. This leads to

$$
\varepsilon \approx \frac{1}{2}\left(\eta_{0} \hat{\phi}\right)^{2}, \quad \eta=-\eta_{0} \quad, \quad \xi=0 .
$$


Hence, the slow roll condition $|\eta|<<1$ requires that $\left|\eta_{0}\right|<<1$.

It follows from Equation (6.2.6) that in the small field case, when $\phi<M_{P}$, then $\varepsilon<<\eta$. In this case it is usual to neglect $\varepsilon$ in the expressions (4.13), (4.16), (4.29) and (4.43) for $r, \alpha_{S}$ and $\alpha_{T}$. This gives

$$
\delta_{n s} \approx 2 \eta_{0}, \quad n_{T}=-\left(\eta_{0} \hat{\phi}\right)^{2}, \quad r=8\left(\eta_{0} \hat{\phi}\right)^{2}, \quad \alpha_{S}=-8 \eta_{0}\left(\eta_{0} \hat{\phi}\right)^{2}, \quad \alpha_{T}=-2 \eta_{0}\left(\eta_{0} \hat{\phi}\right)^{2}
$$

With this approximation there is no running of the spectral index of the scalar fluctuation spectrum, $\alpha_{S}=0$. For this model the Planck result $\delta_{n s}=0.032$ gives $\eta_{0} \approx 0,016$.

With the potential (6.2.1) the number of e-folds is approximately given by

$$
N \approx \frac{M^{4}}{M_{P}^{2}} \int_{\phi_{f}}^{\phi} \frac{1}{V^{\prime}} d \phi=\frac{1}{\eta_{0}} \ln \frac{\phi_{f}}{\phi} .
$$

The end of the slow roll era is given by $\varepsilon\left(\phi_{f}\right)=1$. With Equation (6.2.6) this gives

$$
\phi_{f}=\frac{\sqrt{2} M_{P}}{\eta_{0}} .
$$

Hence

$$
\phi=\frac{\sqrt{2} M_{P}}{\eta_{0}} e^{-\eta_{0} N}
$$

Inserting this into Equation (6.2.6) gives

$$
\varepsilon \approx e^{-2 \eta_{0} N}
$$

The tensor-to-scalar ratio $r$, the spectral index $n_{T}$ and its running $\alpha_{T}$ for this model are

$$
r \approx 16 e^{-2 \eta_{0} N}=16 e^{-\delta \delta_{n S} N}, \quad n_{T}=-2 e^{-2 \eta_{0} N} \quad, \quad \alpha_{S}=-16 \eta_{0} e^{-2 \eta_{0} N}, \quad \alpha_{T}=-4 \eta_{0} e^{-2 \eta_{0} N} .
$$

For $n_{S}=0.096,50<N<60$ this gives $2.2<r<3.2$ which is much larger than the value $r<0.04$ suggested by the BPK-data even when foreground dust is neglected, and $-0.3<n_{T}<-0.2$, $-0.04<\alpha_{S}<-0.02, \quad-0.01<\alpha_{T}<-0.007$.

We have seen that the small field Hilltop inflation does not give a realistic scenario. Let us therefore investigate the large field Hilltop inflation scenario in the remainder of this section. Hence, we shall now present a more accurate calculation, not making the assumption $\phi / M_{P}<<1$. Then it is useful to write the potential as

$$
V=M^{4}\left(1-\widetilde{\phi}^{2}\right), \quad \widetilde{\phi}=\sqrt{\frac{\eta_{0}}{2}} \hat{\phi} .
$$

Here $\phi$ is the magnitude of the inflaton field, while $\widetilde{\phi}$ is a convenient, dimensionless field variable. The slow roll parameters are

$$
\varepsilon=\frac{\eta_{0} \widetilde{\phi}^{2}}{\left(1-\widetilde{\phi}^{2}\right)^{2}} \quad, \quad \eta=-\frac{\eta_{0}}{1-\widetilde{\phi}^{2}}
$$

These expressions imply that

$$
\varepsilon-\eta=\frac{\eta_{0}}{\left(1-\widetilde{\phi}^{2}\right)^{2}}
$$

This shows that $\varepsilon>\eta$ in large field Hilltop inflation, which is opposite to the small field Hilltop inflation. Combining this with the second of the Equation (6.2.14) we have

$$
\eta_{0}=\frac{\eta^{2}}{\varepsilon-\eta}
$$


which confirms that $\eta_{0}<<1$. From the expressions (5.2.14) it also follows that

$$
\varepsilon=\widetilde{\phi}^{2} \eta^{2} / \eta_{0}
$$

The 'spectral index' $\delta_{n s}$ and the tensor-to-scalar ratio $r$ are

$$
\delta_{n s}=2 \eta_{0} \frac{1+2 \widetilde{\phi}^{2}}{\left(1-\widetilde{\phi}^{2}\right)^{2}} \quad, \quad r=16 \eta_{0} \frac{\widetilde{\phi}^{2}}{\left(1-\widetilde{\phi}^{2}\right)^{2}} .
$$

Hence

$$
r=\frac{8 \widetilde{\phi}^{2}}{1+2 \widetilde{\phi}^{2}} \delta_{n s}
$$

This relationship is shown graphically in Figure 5.

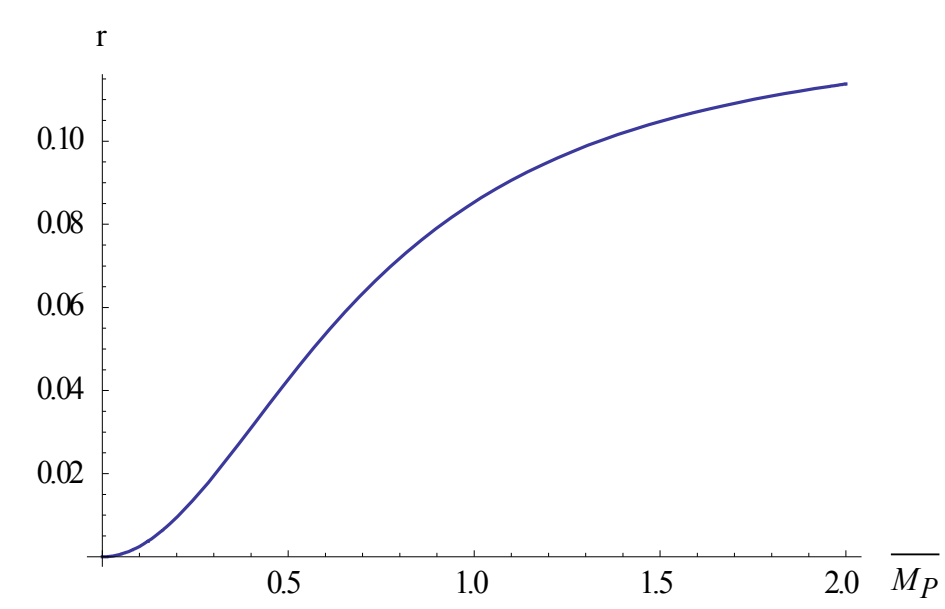

Figure 5. The tensor-to-scalar ratio as a function of the magnitude of the inflaton field of the Hilltop inflation for $n_{S}=0.968, \eta_{0}=0.016$ and $0<\widetilde{\phi}<2 M_{P}$. For larger inflaton field $r$ approaches the value 0.128 .

This equation is valid for arbitrary values of $\widetilde{\phi}$ while Equation (6.2.17) is valid only for $\widetilde{\phi}^{2}<<1$. Equation (6.2.19) may be written

$$
\widetilde{\phi}^{2}=\frac{r / 2}{4 \delta_{n s}-r}
$$

which requires $r<4 \delta_{n s}$. The Planck and BICEP2 data, $\delta_{n s}=0.032$ and $r=0.05$ corresponds to the largest allowed value of $r$ in this inflationary model. The BICEP2/Planck data give $\widetilde{\phi}=0.56$.

It may be noted that this model will be ruled out if one discovers a large running of the tensor-to-scalar ration. This is seen as follows. Equations (4.50) and (6.2.19) gives

$$
\alpha_{r}=\frac{1+\widetilde{\phi}^{2}}{1+2 \widetilde{\phi}^{2}} \delta_{n s}
$$

or

$$
\widetilde{\phi}^{2}=-\frac{\alpha_{r}-\delta_{n s}}{2 \alpha_{r}-\delta_{n s}}
$$

which requires $(1 / 2) \delta_{n s}<\alpha_{r}<\delta_{n s}$. With $\delta_{n s}=0.032$ the running of the tensor-to-scalar ration must obey $0.016<\alpha_{r}<0.032$. Hence, for example the Planck-BICEP-2014 result would rule out this class of inflationary models. 
The number of e-folds is

$$
N=\int_{\phi_{f}}^{\phi} \frac{V}{V^{\prime}} d \phi=\frac{1}{2 \eta_{0}}\left(\widetilde{\phi}^{2}-\widetilde{\phi}_{f}^{2}\right)-\frac{1}{\eta_{0}} \ln \frac{\widetilde{\phi}}{\widetilde{\phi}_{f}}
$$

where $\phi_{f}$ is the final value of the inflaton potential at the end of the slow roll era. The value of $\widetilde{\phi}_{f}$ is usually determined by the condition $\varepsilon\left(\widetilde{\phi}_{f}\right)=1$ which gives

$$
\widetilde{\phi}_{f}=\frac{1}{1-\sqrt{\eta_{0}}} \approx 1
$$

The corresponding final value of the inflaton field is

$$
\phi_{f}=\sqrt{\frac{2}{\eta_{0}}} \frac{M_{P}}{1-\sqrt{\eta_{0}}} \approx \sqrt{\frac{2}{\eta_{0}}} M_{P}
$$

which is much larger than the Planck energy.

We now assume that the initial value of the inflaton field is much smaller than the final value so that the first term at the right hand side of Equation (6.2.23) can be neglected. Solving the resulting equation with respect to $\widetilde{\phi}$ and using Equation (6.2.24) we get

$$
\widetilde{\phi}=\widetilde{\phi}_{f} e^{-(1 / 2)\left(1+3 f_{N}\right)} \quad, \quad f_{N}=(2 / 3) \eta_{0} N
$$

Inserting this into the expressions (6.2.18) and using the approximation $\phi_{f} \approx 1$, gives

$$
\delta_{n s}=\frac{\eta_{0}\left(e^{1+3 f_{N}}+2\right)}{2 \sinh ^{2}\left[\frac{1}{2}\left(1+3 f_{N}\right)\right]} \quad, \quad r=\frac{4 \eta_{0}}{\sinh ^{2}\left[\frac{1}{2}\left(1+3 f_{N}\right)\right]}
$$

which is different from the expressions in Equation (8) in Basilakos et al. [95]. It follows from the expressions in Equation (6.2.27) that

$$
e^{1+3 f_{N}}=(2 / r)\left(4 \delta_{n s}-r\right)
$$

or

$$
r=\frac{4 \delta_{n s}}{1+(1 / 2) e^{1+3 f_{N}}}
$$

Hence, there is a linear relationship between $r$ and $n_{S}$ in this inflationary model. Since $e^{1+(1 / 3) f_{N}}>1$, Equation (6.2.29) requires that (using $\delta_{n s}=0.032$ )

$$
r<(8 / 3) \delta_{n s}=0.085
$$

This is the main prediction of the large field hilltop model. It is in agreement with the BICEP2/Planck data.

Inserting the expression (6.2.28) into the second of the expressions (6.2.27) gives $\eta_{0}$ in terms of observable quantities

$$
\eta_{0}=\frac{1}{32} \frac{\left(8 \delta_{n s}-3 r\right)^{2}}{4 \delta_{n s}-r}
$$


Seemingly this allows a large value of $\eta_{0}$ since $0<\eta_{0}<\infty$ for $(8 / 3) \delta_{n s}<r<4 \delta_{n s}$. However, as was shown in Equation (6.2.30), this region of $r$ is not allowed. Inserting the expression (4.2.29) for $r$ into Equation (6.2.31) gives

$$
\eta_{0}=\frac{\delta_{n s}}{8} \frac{\left[1-4 e^{-\left(1+3 f_{N}\right)}\right]^{2}}{1+2 e^{-\left(1+3 f_{N}\right)}} .
$$

This shows that $\eta_{0}<(1 / 8) \delta_{n s}$. With the Planck value this gives $\eta_{0}<0.004$.

Basilakos et al. [95] have considered the case $n=2, N=50$, giving $f_{N}=10^{3}$. However, the model in combination with the Planck results does not permit so large value of $n$. Inserting Equation (6.2.19) into Equation (6.2.26) and then using Equation (6.2.14) we have

$$
f_{N}=\frac{2}{3} \frac{N \eta^{2}}{\varepsilon-\eta}=\frac{4}{3} \frac{N M_{P}^{2} \eta}{\phi^{2} \eta-2 M_{P}^{2}}
$$

showing again that in this inflationary model the inflaton field must be large, $\phi>\sqrt{2 / \eta} M_{P}$.

A more general form of the Hilltop inflaton potential has also been considered (Kinney et al. [88,96], Zarei [97] and Barenboim et al. [50]),

$$
V(\phi) \approx V_{0}\left(1-\widetilde{\phi}^{p}\right), \quad \widetilde{\phi}=\phi / \phi_{0}, \quad p \neq 1 \& p \neq 2,
$$

where $\phi_{0}$ is the value of the inflaton field at the extremum of the potential. It is usually assumed that $\widetilde{\phi}<<1$. We shall first consider this case, which permits the approximation $V \approx V_{0}$ in the numerator in the expressions for the slow roll parameters. This gives

$$
\varepsilon=\frac{p^{2}}{2} \hat{\phi}_{0}^{-2} \widetilde{\phi}^{2(p-1)}, \quad \eta=-p(p-1) \hat{\phi}_{0}^{-2} \widetilde{\phi}^{p-2}, \quad \xi=p^{2}(p-1)(p-2) \hat{\phi}_{0}^{-4} \widetilde{\phi}^{2(p-2)},
$$

where $\hat{\phi}_{0}=\phi_{0} / M_{P}$. Hence $\varepsilon<<\eta$. We shall now assume that $p \neq 1$ and $p \neq 2$. Then it follows that

$$
\xi=\frac{p-2}{p-1} \eta^{2}
$$

Inserting the expression (6.2.35) into Equations (4.13) and (4.29) we get

$$
\begin{aligned}
& \delta_{n s}=2 p(p-1) \hat{\phi}_{0}^{-2} \widetilde{\phi}^{p-2}, \quad r=8 p^{2} \hat{\phi}_{0}^{-2} \widetilde{\phi}^{2(p-1)}, \\
& \alpha_{S}=-2 p^{2}(p-1)(p-2) \hat{\phi}_{0}^{-4} \widetilde{\phi}^{2(p-2)}
\end{aligned}
$$

It follows that for this class of inflationary models the $\left(\delta_{n s}, r\right)$ and $\left(\delta_{n s}, \alpha_{S}\right)$ relationships can be written

$$
r \approx \frac{4 p}{p-1} \frac{\delta_{n s}^{\frac{2(p-1)}{p-2}}}{\left[2 p(p-1) \hat{\phi}_{0}^{2}\right]^{\frac{p}{p-2}}} \quad, \quad \alpha_{S}=-\frac{1}{2} \frac{p-2}{p-1} \delta_{n s}^{2} .
$$

Hence that $r$ has a value close to zero in these inflation models for $p>1$.

The number of e-folds is

$$
N=\frac{1}{M_{P}^{2}} \int_{\phi_{f}}^{\phi} \frac{V}{V^{\prime}} d \phi=-\frac{\phi_{0}^{p}}{p M_{P}^{2}} \int_{\phi_{f}}^{\phi} \phi^{1-p} d \phi=\frac{\hat{\phi}_{0}^{2}}{p(p-2)}\left(\frac{1}{\widetilde{\phi}^{p-2}}-\frac{1}{\widetilde{\phi}_{f}^{p-2}}\right) .
$$

We shall here consider a slow roll era with $\widetilde{\phi}<<\widetilde{\phi}_{f}$, so we can neglect the last term in Equation (6.2.39), which gives

$$
\hat{\phi}_{0}^{2}(\widetilde{\phi})^{2-p} \approx p(p-2) N
$$


Hence

$$
\eta \approx-\frac{p-1}{p-2} \frac{1}{N} \quad, \quad \xi \approx \frac{p-2}{p-1} \frac{1}{N^{2}}
$$

From this we get $[98,99]$

$$
r=\frac{2 \hat{\phi}_{0}^{\frac{2 p}{p-2}}}{[p(p-2) N]^{\frac{2(p-1)}{p-2}}} \rightarrow \frac{8 p^{2} \hat{\phi}_{0}^{\frac{2 p}{p-2}}}{[p(p-2) N]^{\frac{2(p-1)}{p-2}}}
$$

It may be noted that for these models it is possible to obtain sufficiently small values by choosing $\hat{\phi}_{0}<<1$, which are then small field models. The $r, \delta_{n s}-$ relation takes the form

$$
r=\left(\frac{\phi_{0}}{2}\right)^{\frac{2 p}{p-2}}\left[\frac{\delta_{n s}}{p(p-1)}\right]^{\frac{2(p-1)}{p-2}} \rightarrow 8 p^{2} \hat{\phi}_{0}^{\frac{2 p}{p-2}}\left[\frac{\delta_{n s}}{2 p(p-1)}\right]^{\frac{2(p-1)}{p-2}}
$$

It follows from the expressions (6.2.42) that the running of the spectral index of scalar fluctuations can be written

$$
\alpha_{S}=-\frac{1}{2} \frac{p-2}{p-1} \delta_{n S}^{2}=-\frac{1}{N} \delta_{n s} .
$$

With $\delta_{n s}=0.032$ and $N=50$ this gives $\alpha_{S}=-0.0006$ which is permitted by the Planck 2015 results. Furthermore we get

$$
p=\frac{2\left(N \delta_{n s}-1\right)}{N \delta_{n s}-2}
$$

giving $p=-3$. Inserting this, together with $\delta_{n s}=0.032$, into Equation (6.2.43) gives $r \approx 3 \times 10^{-5} \phi_{0}^{1.2}$. For $\hat{\phi}_{0}<<1$ this gives a very small value the tensor-to-scalar ratio.

Again we shall make a more accurate calculation, this time not making the assumption $\widetilde{\phi}<<1$. Then the slow roll parameters are

$$
\begin{aligned}
\varepsilon & =\frac{p^{2}}{2} \hat{\phi}_{0}^{-2} \frac{\widetilde{\phi}^{2(p-1)}}{\left(1-\widetilde{\phi}^{p}\right)^{2}}, \quad \eta=-p(p-1) \hat{\phi}_{0}^{-2} \frac{\widetilde{\phi}^{p-2}}{1-\widetilde{\phi}^{p}}, \\
\xi & =p^{2}(p-1)(p-2) \hat{\phi}_{0}^{-4} \frac{\widetilde{\phi}^{2(p-2)}}{\left(1-\widetilde{\phi}^{p}\right)^{2}}
\end{aligned}
$$

It follows from these expressions that

$$
\xi=\frac{p-2}{p-1} \eta^{2}
$$

The corresponding differential equation is

$$
V^{\prime} V^{\prime \prime \prime}=\frac{p-2}{p-1} V^{\prime \prime 2}
$$

with general solution

$$
V(\phi)=(A \phi+B)^{p}+C,
$$

where $A, B$ and $C$ are arbitrary constants. This generalizes the potential (6.2.34).

J. Garcia-Bellido and D. Roest [70] have called the corresponding inflationary universe model with negative value of $p$ for the inverse hilltop model, while Shiu and Tye [100] and Drees et al. [101] have called it inverse power law inflation. Such a model has also been investigated by Z. Lu [102] with $p=-2$. Steer and Vernizzi [28] have considered an inflationary model with inverse power law potential,

$$
V(\hat{\phi})=\frac{V_{0}}{1+\hat{\phi}^{4}}
$$


With the approximation (4.2.5) their predictions are $\delta_{n s}=3 / N$, and the $\delta_{n s}, r$-relation is $r=(1 / 3) \widetilde{\phi}^{4} \delta_{n s}$.

\subsection{Symmetry Breaking Inflation}

There exists a so-called symmetry breaking inflation model with potential (Chiba and Khori, 2014)

$$
V(\phi)=M^{4}\left(\phi^{2}-M^{2}\right)^{2} .
$$

This has also been called double-well inflation by Martin et al. (2013), hilltop inflation by Chiba and Khori [89], topological inflation by Chung and Lin [103], and Higgs-like by and Escudero et al. [104]. Here $M$ represents a symmetry breaking energy scale where the potential has a minimum. In the weak field case the potential (6.3.1) is often called a Higgs potential (Rehman et al. [105], but this term will here be reserved for the potential in Section 6.7.

Inserting the potential (6.3.1) into Equation (2.17) and integrating gives the time evolution of the inflaton field during the slow roll era

$$
\phi=\phi_{0} e^{4 M_{P} \sqrt{V_{0} / 3} t} .
$$

which is similar to that of polynomial inflation with $p=4$.

For the potential (6.3.1) the slow roll parameters are

$$
\varepsilon=\frac{8 M_{P}^{2} \phi^{2}}{\left(\phi^{2}-M^{2}\right)^{2}} \quad, \quad \eta=\frac{4 M_{P}^{2}\left(3 \phi^{2}-M^{2}\right)}{\left(\phi^{2}-M^{2}\right)^{2}} \quad, \quad \xi=\frac{96 M_{P}^{4} \phi^{2}}{\left(\phi^{2}-M^{2}\right)^{3}} .
$$

The number of e-folds for this model is (Qiu, 2014)

$$
N=\frac{1}{8 M_{P}^{2}}\left(\phi^{2}-\phi_{f}^{2}\right)-\frac{M^{2}}{4 M_{P}^{2}} \ln \frac{\phi}{\phi_{f}}
$$

where the final value of the inflaton field is determined by $\varepsilon\left(\phi_{f}\right)=1$ which leads to

$$
\phi_{f}^{2}=4 M_{P}^{2}+M^{2} \pm \sqrt{16 M_{P}^{4}+8 M_{P}^{2} M^{2}} .
$$

There is a consistency relation for this model,

$$
\xi=3 \varepsilon(\eta-\varepsilon) .
$$

Inserting the expressions (3.1) for the slow roll parameters leads to the differential equation

$$
\frac{V V^{\prime \prime \prime}}{V^{\prime 2}}-\frac{3}{2} \frac{V^{\prime \prime}}{V^{\prime}}+\frac{3}{4} \frac{V^{\prime}}{V}=0,
$$

which may be written

$$
\left(V^{1 / 2}\right)^{\prime \prime \prime}=0 .
$$

Hence the general form of the potential for mono-field inflationary models fulfilling the consistency condition (6.3.6) is

$$
V=\left(A \phi^{2}+B \phi+C\right)^{2} .
$$

where $A, B, C$ are integration constants. 
Inserting the relation (6.3.6) into Equation (4.29) and using Equation (4.24) gives

$$
\alpha_{S}=\frac{r}{64}\left(3 r-20 \delta_{n S}\right) \text {. }
$$

With $\delta_{n s}=0.032$ and $r=0.05$ we get $\alpha_{S}=-0.0004$ which is permitted by the Planck 2015 data giving $\alpha_{S}=0.003 \pm 0.007$ [35].

Using the expressions (6.3.3), we find that $\delta_{n s}$ and $r$ are

$$
\delta_{n s}=8 M_{P}^{2} \frac{3 \phi^{2}+M^{2}}{\left(\phi^{2}-M^{2}\right)^{2}} \quad, \quad r=128 M_{P}^{2} \frac{\phi^{2}}{\left(\phi^{2}-M^{2}\right)^{2}} .
$$

It follows that

$$
r=4\left(\delta_{n s}-3 x^{2}+x \sqrt{2 \delta_{n s}+9 x^{2}}\right) \quad, \quad x=M_{P} / M
$$

A plot of this relationship for $\delta_{n s}=0.032$ and $0<x<0.1$ is shown in Figure 6 .

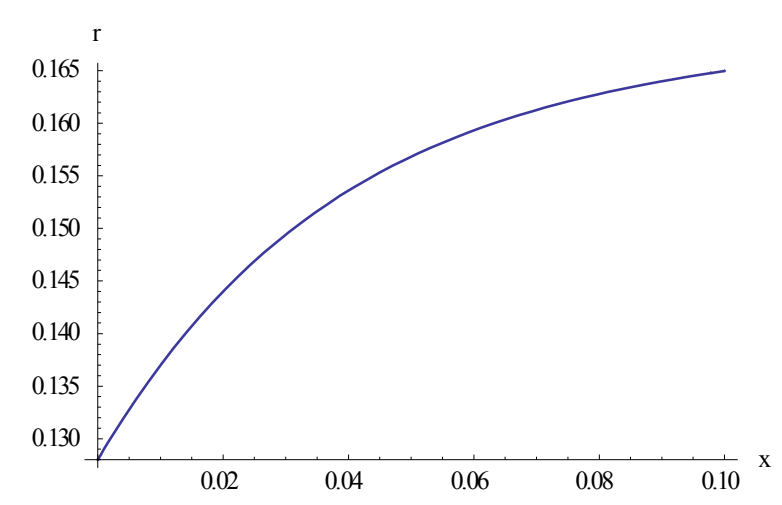

Figure 6. Predicted values of $r$ given in Equation (6.3.12) as a function of $M$ for $M>10 M_{P}$ and $n_{S}=0.968$.

Equation (6.3.10) gives $\lim _{x \rightarrow \infty} r=5 \delta_{n s}$. Hence this inflationary model predicts that $4 \delta_{n s}<r<5 \delta_{n s}$ or with the Planck data, $0.128<r<0.16$. This is larger than the values, $r<0.04$, favored by the BPK-data.

Solving Equation (6.3.12) with respect to $M$ we find for the symmetry breaking inflationary models

$$
M=2 \sqrt{2} M_{P} \frac{\sqrt{16 \delta_{n s}-3 r}}{4 \delta_{n s}-r}
$$

Here $\delta_{n s}=0.032, r=0.04$ gives $M \approx 20 M_{P}$. Hence this is an example of large field inflation.

The potential (6.3.1) can be generalized by including a non-minimal coupling to the Ricci scalar [104]. In this case the potential takes the form

$$
V(\phi)=\frac{M^{4}\left(\phi^{2}-M^{2}\right)^{2}}{\left(1+\xi \hat{\phi}^{2}\right)^{2}},
$$

where $\xi$ is the coupling constant. Escudero et al. [104] have shown that this model gives agreement with the Planck data if $\xi>100$.

\subsection{Exponential Potential and Power Law Inflation}

In these inflationary universe models the potential is an exponential function of the scalar field [106],

$$
V(\phi)=M^{4} e^{-\lambda \hat{\phi}}
$$


Inserting this potential into Equation (2.17) and integrating with $\phi(0)=0$ gives the time dependence of the inflaton field

$$
\phi=\frac{2 M_{P}}{\lambda} \ln \left(1+\frac{\lambda^{2}}{6} \frac{M^{2}}{M_{P}} t\right)
$$

With this expression in Equation (6.4.1) we get the time dependence of the potential

$$
V(t)=\frac{M^{4}}{\left(1+\frac{\lambda^{2}}{6} \frac{M^{2}}{M_{P}} t\right)^{2}} .
$$

Differentiating Equation (6.4.2) gives

$$
\dot{\phi}=\frac{(\lambda / 3) M^{2}}{1+\frac{\lambda^{2}}{6} \frac{M^{2}}{M_{P}} t} \quad, \quad \ddot{\phi}=\frac{\lambda^{3} M^{4}}{18 M_{P}\left(1+\frac{\lambda^{2}}{6} \frac{M^{2}}{M_{P}} t\right)^{2}} .
$$

Inserting Equation (6.4.3) and the first of the expressions (6.4.4) into Equation (2.2) gives

$$
H=\frac{M^{2}}{\sqrt{3} M_{P}} \frac{\sqrt{1+\frac{\lambda^{2}}{18}}}{1+\frac{\lambda^{2}}{6} \frac{M^{2}}{M_{P}} t} .
$$

This gives for the rate of roll,

$$
\frac{\ddot{\phi}}{H \dot{\phi}}=\sqrt{\frac{3}{2\left(18+\lambda^{2}\right)}} \lambda^{2},
$$

which is constant. The class of inflationary models with a constant rate of roll has been further studied by Motohashi et al. [107].

Integrating Equation (6.4.5) with $a(0)=a_{0}$ shows that the scale factor is a power function of the cosmic time,

$$
a(t)=a_{0}\left(1+\frac{\lambda^{2}}{6} \frac{M^{2}}{M_{P}} t\right)^{\frac{2}{\lambda^{2}} \sqrt{1+\frac{\lambda^{2}}{18}}}
$$

Therefore this inflationary model has been called power law inflation [108].

Amorós and Haro [56] have noted that the potential of these inflationary models obey the condition $\xi=\eta^{2}$. Inserting the definitions (3.1) for $\xi$ and $\eta$ we get the differential equation

$$
V^{\prime} V^{\prime \prime \prime}-V^{\prime \prime 2}=0
$$

with general solution

$$
V(\phi)=A e^{B \phi}+C
$$

where $A, B$ and $C$ are constants of integration.

For this class of inflationary models the slow roll parameters are independent of the duration of the inflation era and have values

$$
\varepsilon=(1 / 2) \lambda^{2}, \eta=\lambda^{2}, \xi=\lambda^{4},
$$

giving

$$
\delta_{n s}=\lambda^{2}, \quad n_{T}=-\lambda^{2}, \quad r=8 \lambda^{2}, \quad \alpha_{S}=0 .
$$

Hence there is no running of the scalar spectral index. Equation (6.4.11) implies the relationship

$$
r=8 \delta_{n s}, n_{T}=-\delta_{n s}
$$


In this model there is no running of the spectral indices or the tensor-to-scalar ratio. With the value $\delta_{n s}=0.032$ from the Planck measurements we get $\lambda^{2}=0.032$ and $r=0.256$. Exponential inflationary models with power law expansion have mainly been considered with values of the exponent $6 / \lambda^{2}$ smaller than 2, which is in conflict with the Planck data. Furthermore the value of $r$ is much too high. Such exponential inflationary models are ruled out by the BPK-data, that indicate $r<0.04$. This type of inflationary models is an example of models that fitted the preliminary BICEP2 result with a high value of $r$ quite well [59], but that are ruled out by the recent combined BPK-results [109].

Furthermore, is should be noted that there is no natural exit of the slow roll era in the original version of the exponential inflation model.

However, in light of the Planck results S. Unnikrishnan and V. Sahni [110] have constructed a revised power law model in which inflation is driven by a scalar field with an inverse power law potential.

Geng et al. [111] have recently generalized these types of inflationary models by including an additional free parameter $p$ so that the potential is given the form

$$
V=V_{0} e^{-\lambda \hat{\phi}^{p}}
$$

Drees et al. [101] have considered an inflationary model with a potential having the same form, but with plus in the exponent. For the potential (6.4.13) the slow roll parameters are

$$
\begin{aligned}
& \varepsilon=(1 / 2) p^{2} \lambda^{2} \hat{\phi}^{2(p-1)} \quad, \quad \eta=-p \lambda \hat{\phi}^{p-2}\left(p-1-p \lambda \hat{\phi}^{p}\right) \\
& \xi=p^{2} \lambda^{2} \hat{\phi}^{2 p-4}\left[(p-1)(p-2)-3 p(p-1) \lambda \hat{\phi}^{p}+p^{2} \lambda^{2} \hat{\phi}^{2 p}\right] .
\end{aligned} .
$$

Hence the scalar tilt $\delta_{n s}$ and the scalar-to-tensor ratio $r$ are

$$
\begin{aligned}
& \delta_{n S}=p^{2} \lambda^{2} \hat{\phi}^{2 p-2}+2 p(p-1) \lambda \hat{\phi}^{p-2}, \quad r=8 p^{2} \lambda^{2} \hat{\phi}^{2 p-2} \\
& \alpha_{S}=-2 p^{2}(p-1) \lambda^{2}\left(\frac{r}{8 p^{2} \lambda^{2}}\right)^{\frac{p-2}{p-1}}\left[p \lambda\left(\frac{r}{8 p^{2} \lambda^{2}}\right)^{\frac{1}{2(p-1)}}-(p-2)\right],
\end{aligned}
$$

for $p \neq 1$. It follows that

$$
\delta_{n s}=\frac{r}{8}+(p-1)(2 p \lambda)^{\frac{1}{p-1}}\left(\frac{r}{2}\right)^{\frac{p-2}{2 p-2}}
$$

and

$$
\alpha_{S}=-(p-1)(p \lambda)^{\frac{2}{p-1}}\left(\frac{r}{8}\right)^{\frac{p-2}{p-1}}\left[(p \lambda)^{\frac{p-2}{p-1}}\left(\frac{r}{8}\right)^{\frac{1}{2(p-1)}}-(p-2)\right] .
$$

Geng et al. [111] have chosen $\lambda=10^{-8}$.

In the special case with $p=2$ we have

$$
\begin{gathered}
V=V_{0} e^{-\lambda \hat{\phi}^{2}} \\
\varepsilon=2 \lambda^{2} \hat{\phi}^{2}, \quad \eta=-2 \lambda\left(1-2 \lambda \hat{\phi}^{2}\right), \quad \xi=8 \lambda^{3} \hat{\phi}^{2}\left(2 \lambda \hat{\phi}^{2}-3\right), \\
\delta_{n s}=4 \lambda\left(\lambda \hat{\phi}^{2}+1\right), \quad r=32 \lambda^{2} \hat{\phi}^{2} \\
\delta_{n s}=\frac{r}{8}+4 \lambda, \quad \alpha_{S}=-\lambda^{2}(2 r)^{1 / 2} .
\end{gathered}
$$

Hence

$$
\lambda=\frac{1}{4}\left(\delta_{n s}-\frac{r}{8}\right) .
$$

Inserting $\delta_{n s}=0.032$ and $r<0.04$ gives the requirement $\lambda>0.007$.

Other members of the class of exponential inflationary models are the $\beta$-exponential inflationary models [112,113]. In these models the usual exponential function in the potential is replaces by the 
general exponential function. This introduces a new parameter which may be adjusted in order that the models shall agree with the observational data.

The hubble slow roll parameters are

$$
\varepsilon_{H}=\frac{\lambda^{2}}{2} \frac{1}{(\beta \lambda \phi-1)^{2}} \quad, \quad \eta_{H}=\frac{\lambda^{2}}{2} \frac{1-2 \beta}{(\beta \lambda \phi-1)^{2}} .
$$

The scalar spectral tilt and the tensor-to-scalar ratio are

$$
\delta_{n s}=\lambda^{2} \frac{1+2 \beta}{(\beta \lambda \phi-1)^{2}} \quad, \quad r=8 \lambda^{2} \frac{1}{(\beta \lambda \phi-1)^{2}} .
$$

Hence

$$
r=\frac{8 \delta_{n s}}{1+2 \beta}
$$

which may be written

$$
\beta=\frac{1}{2}\left(8 \frac{\delta_{n s}}{r}-1\right)
$$

Inserting $\delta_{n s}=0.032$ and $r<0.04$ gives $\beta=2.7$.

The value of the inflaton field, $\phi_{f}$, is given by $\varepsilon_{H}\left(\phi_{f}\right)=1$ which leads to

$$
\phi_{f}=\frac{1}{\beta}\left(\frac{1}{\lambda}-\frac{1}{\sqrt{2}}\right) .
$$

For $\beta \neq 0$ the number of e-folds of the inflationary era is

$$
N=\int_{\phi_{f}}^{\phi} \frac{d \phi}{\sqrt{2 \varepsilon_{H}(\phi)}}=\frac{1}{\lambda}\left(\frac{1}{2} \beta \lambda \phi^{2}-\phi\right)+\frac{1}{2 \beta}\left(\frac{1}{\lambda^{2}}-\frac{1}{2}\right) .
$$

Inserting this into Equation (6.4.24) some nice cancellations happen, which lead to very simple, $\lambda$ - independent expressions for the spectral parameters in terms of the number of e-folds,

$$
\delta_{n s}=\frac{2(1+2 \beta)}{4 \beta N+1} \quad, \quad r=\frac{16}{4 \beta N+1} .
$$

The last of these equations can be written

$$
\beta=\frac{16-r}{4 N r} \text {. }
$$

Inserting $N=50, r=0.04$ gives $\beta \approx 2$. It follows from Equations (6.4.26) and (6.4.30) that

$$
r=16 \frac{N \delta_{n s}-1}{2 N-1}
$$

With $N=50, \delta_{n s}=0,32$ we get $r \approx 0.10$ which is a little larger than permitted by the Planck data.

\subsection{Natural Inflation}

The original natural inflation potential was presented by K. Freese et al. [114] and has been further developed and compared with observational data by Freese and Kinney $[115,116]$. In the original model there are two variants of the potential of the scalar field generating the dark energy, given by

$$
V_{-}(\phi)=V_{0}(1-\cos \widetilde{\phi})=2 V_{0} \sin ^{2}(\widetilde{\phi} / 2) \quad, \quad V_{+}(\phi)=V_{0}(1+\cos \widetilde{\phi})=2 V_{0} \cos ^{2}(\widetilde{\phi} / 2)
$$


Here $\widetilde{\phi}=\phi / M$, and $M$ is the spontaneous symmetry breaking scale. In order for inflation to occur, we must have $M>M_{P}$ [117]. The constant $V_{0}$ is a characteristic energy scale for the model. The potential $V_{-}$has a minimum at $\widetilde{\phi}=0$ and $V_{+}$at $\widetilde{\phi}=\pi$.

Inserting the potential $V_{+}$into Equation (2.17) and integrating we find the time evolution of the inflaton field during the slow roll era,

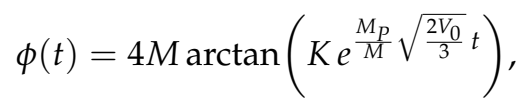

where $K$ is an integration constant. We here chose $K=1$. Then $\widetilde{\phi}_{0}=\widetilde{\phi}(0)=\pi$. This boundary condition will be chosen both for $V_{-}(\phi)$ and $V_{+}(\phi)$. Then $V_{-}\left(\phi_{0}\right)=2 V_{0}$ and $V_{+}\left(\phi_{0}\right)=0$.

Using the identity

$$
\cos (4 \theta)=1-\frac{8 \tan ^{2} \theta}{\left(1+\tan ^{2} \theta\right)^{2}}
$$

the time evolution of the potential $V_{-}$is found to be

$$
V_{-}(t)=\frac{2 V_{0}}{\cosh ^{2}\left(\frac{M_{P}}{M} \sqrt{\frac{2 V_{0}}{3}} t\right)} .
$$

Differentiating the expression (6.5.2) gives

$$
\dot{\widetilde{\phi}}=\frac{2 M_{P} \sqrt{2 V_{0} / 3}}{\cosh \left(\frac{M_{P}}{M} \sqrt{\frac{2 V_{0}}{3}} t\right)} .
$$

The slow roll era starts at a flat upper part of the curve, for example at $\phi=0$ (small field inflation) or at $\phi=2 \pi M$ (large field inflation). The slow roll parameters are (with the upper sign corresponding to $V_{-}$)

$$
\varepsilon_{ \pm}=\frac{b}{2} \frac{1 \pm c_{\phi}}{1 \mp c_{\phi}}, \quad \eta_{ \pm}=\mp b \frac{c_{\phi}}{1 \mp c_{\phi}}, \quad \xi_{ \pm}=-b^{2} \frac{1 \pm c_{\phi}}{1 \mp c_{\phi}}, \quad b=\left(\frac{M_{P}}{M}\right)^{2}, \quad c_{\phi} \equiv \cos \widetilde{\phi} .
$$

where $\phi$ is the initial value of the field giving rise to $N$ e-folds. The parameter $b$ represents the symmetry breaking scale and $b<1$ for $M>M_{P}$. It follows from these relations that

$$
c_{\phi}= \pm \frac{\eta_{ \pm}}{\eta_{ \pm}-2 \varepsilon_{ \pm}} .
$$

The expressions (6.5.6) may be given the alternative forms

$$
\begin{aligned}
& \varepsilon_{-}=\frac{b}{2}\left(\frac{s_{\phi}}{1-c_{\phi}}\right)^{2}=\frac{b}{2} \operatorname{cotan}^{2} \frac{\widetilde{\phi}}{2}, \quad \varepsilon_{+}=\frac{b}{2}\left(\frac{s_{\phi}}{1+c_{\phi}}\right)^{2}=\frac{b}{2} \tan ^{2} \frac{\widetilde{\phi}}{2}, \quad s_{\phi} \equiv \sin \widetilde{\phi} \\
& \eta_{-}=\frac{b}{2}\left(\cot \operatorname{con}^{2} \frac{\widetilde{\phi}}{2}-1\right)=\varepsilon_{-}-\frac{b}{2}, \quad \eta_{+}=\frac{b}{2}\left(\tan ^{2} \frac{\widetilde{\phi}}{2}-1\right)=\varepsilon_{+}-\frac{b}{2}, \\
& \xi_{-}=-b^{2} \operatorname{cotan}^{2} \frac{\widetilde{\phi}}{2}=-2 b \varepsilon_{-}, \quad \xi_{+}=-b^{2} \tan ^{2} \frac{\widetilde{\phi}}{2}=-2 b \varepsilon_{+}
\end{aligned}
$$

For both signs the expressions (6.5.3) give

$$
c_{\phi}^{2}=\frac{\eta^{2}}{\eta^{2}+2 b \varepsilon},
$$

and the consistency relations

$$
\eta-\varepsilon=-b / 2, \xi=-b \varepsilon
$$


and

$$
\xi=4 \varepsilon(\eta-\varepsilon) .
$$

From the last relation and Equation (4.29) we have

$$
\alpha_{S}=8 \varepsilon(\eta-2 \varepsilon)
$$

Inserting the expressions (4.24) for $\varepsilon$ and $\eta$ gives a relation between observables for the natural inflation models

$$
\alpha_{S}=\frac{r}{32}\left(r-8 \delta_{n s}\right)
$$

It follows from Equations (4.48), (4.50) and (6.5.10) that for this class of inflationary universe models

$$
\alpha_{T}=\frac{\alpha_{S}}{2}=-\frac{\alpha_{r}}{8}=\frac{r}{64}\left(r-8 \delta_{n s}\right) .
$$

With $\delta_{n s}=0.032$ and $r=0.05$ we get $\alpha_{S}=-0.00032, \alpha_{T}=-0.00016$.

Inserting the expressions for $\varepsilon$ and $\eta$ in Equation (3.1) into the first of Equation (6.5.10) gives the differential equation

$$
\frac{V^{\prime \prime}}{V}-\frac{1}{2} \frac{V^{\prime 2}}{V^{2}}=-\frac{1}{2 f^{2}}
$$

which may be written

$$
\left(V^{1 / 2}\right)^{\prime \prime}+(1 / 2 f)^{2} V^{1 / 2}=0
$$

The general solution is

$$
V(\phi)=(A \cos (\hat{\phi} / 2)+B \sin (\hat{\phi} / 2))^{2} .
$$

The two potentials in Equation (6.5.1) corresponds to the special cases $A=\sqrt{2 V_{0}}, \quad B=0$ and $A=0, B=\sqrt{2 V_{0}}$, respectively.

The last of the Equation (6.5.10) on the other hand, corresponds to the differential equation

$$
V^{\prime \prime \prime}=-\left(1 / M^{2}\right) V^{\prime}
$$

Integration gives

$$
V^{\prime \prime}+\left(1 / M^{2}\right) V=A,
$$

where $A$ is an integration constant. The general solution is

$$
V(\phi)=A M^{2}+B \sin (\widetilde{\phi})+C \cos (\widetilde{\phi}),
$$

where $A, B, C$ are integration constants.

Equation (6.5.11) leads to

$$
\frac{V V^{\prime \prime \prime}}{V^{\prime 2}}=2 \frac{V^{\prime \prime}}{V^{\prime}}-\frac{V^{\prime}}{V},
$$

which may be written

$$
\frac{\left(V^{1 / 2}\right)^{\prime \prime \prime}}{\left(V^{1 / 2}\right)^{\prime \prime}}=\frac{\left(V^{1 / 2}\right)^{\prime}}{V^{1 / 2}} .
$$

Integration gives

$$
\left|\frac{\left(V^{1 / 2}\right)^{\prime \prime}}{V^{1 / 2}}\right|=K
$$


where $K$ is a positive constant. Hence,

$$
\left(V^{1 / 2}\right)^{\prime \prime} \pm K V^{1 / 2}=0
$$

With $K=1 / M^{2}$ and the plus sign this is identical to Equation (6.5.16). With the minus sign the trigonometric functions in Equation (6.5.17) are replaced by hyperbolic functions.

The spectral parameters are

$$
\delta_{n s \pm}=b \frac{3 \pm c_{\phi}}{1 \mp c_{\phi}} \quad, \quad n_{T \pm}=-b \frac{1 \pm c_{\phi}}{1 \mp c_{\phi}}, \quad r_{ \pm}=8 b \frac{1 \pm c_{\phi}}{1 \mp c_{\phi}} .
$$

It follows from these equations that

$$
r=4\left(\delta_{n s}-b\right) \quad, \quad c_{\phi}= \pm \frac{8 \delta_{n s \pm}-3 r_{ \pm}}{8 \delta_{n s \pm}-r_{ \pm}} \quad, \quad n_{T}=-\frac{r}{8}
$$

where the relationship for $n_{T}$ is the same as Equation (4.16). Since $-1 \leq c_{\phi} \leq 1$ and $b>0$ these equations require $0 \leq r \leq 4 \delta_{n s}$. With the value $\delta_{n s}=0.032$ from the Planck measurements, a prediction of the natural inflation model is that $r \leq 0.128$. Inserting the BICEP2/Planck value $r=0.05$ gives $c_{\phi}=\mp 0.5$.

The symmetry breaking parameter $b$ can be determined from observations from the first of the relationships (6.5.26),

$$
b=\delta_{n s}-\frac{r}{4}
$$

With $\delta_{n s}=0.032$ and $r=0.05$ we have $b=0.02$, giving $M=7 M_{P}$. Hence the symmetry breaking energy is much higher than the Planck energy.

Using successively Equations (4.29), (4.24), and (6.5.10) the slow roll parameter $\xi$ can also be determined from observations,

$$
\xi=-\frac{r}{32}\left(4 \delta_{n s}-r\right)
$$

Hence, for $r<4 \delta_{n s}$ this parameter is negative. For the BICEP2/Planck data we get $\xi=-0.0001$. The number of e-folds, with + and - , respectively, in Equation (6.5.1), are

$$
N_{+}=\frac{2}{b} \ln \left|\frac{\sin \left(\widetilde{\phi}_{f} / 2\right)}{\sin (\widetilde{\phi} / 2)}\right| \quad N_{-}=\frac{2}{b} \ln \left|\frac{\cos \left(\widetilde{\phi}_{f} / 2\right)}{\cos (\widetilde{\phi} / 2)}\right| .
$$

It is usual to specify that the inflationary era ends when $\varepsilon=1$. Inserting $\phi=\phi_{f}$ in the first of the Equation (6.5.1) with $\varepsilon=1$ we get

$$
\cos \left(\widetilde{\phi}_{f \pm}\right)= \pm \frac{2-b}{2+b}
$$

Inserting this into the two expressions (6.5.29) gives

$$
c_{\phi \pm}= \pm\left(1-\frac{4}{2+b} e^{-b N}\right)
$$

or

$$
\sin \left(\widetilde{\phi}_{\phi+} / 2\right)=\cos \left(\widetilde{\phi}_{\phi-} / 2\right)=\sqrt{2 /(2+b)} e^{-(b / 2) N} .
$$

Hence $\widetilde{\phi}_{\phi+}+\widetilde{\phi}_{\phi-}=\pi$. It follows from Equations (6.5.25) and (6.5.32) that

$$
\delta_{n s}=b \frac{(2+b) e^{b N}+2}{(2+b) e^{b N}-2}, \quad r=\frac{16 b}{(2+b) e^{b N}-2} .
$$


This shows that the models with + and - in the formula (4.5.1) for the potential have the same empirical contents. These expressions are in agreement with the first of the Equation (4.5.23).

We have two limits [99],

A. $\quad b N>>1$. Then $\delta_{n s} \approx b$ and $r \approx[16 b /(2+b)] \exp (-b N)$. This is similar to the Hilltop predictions (4.2.7) and (4.2.12). In this limit $\delta_{n s}>>1 / N \approx 0.02$ which is not favored by the Planck measurements.

B. $\quad b N<<1$. Then $\delta_{n s} \approx 2 / N$ and $r \approx 8 / N \approx 4 \delta_{n s}$. This is similar to the predictions of chaotic inflation with $p=2$. With the Planck value $\delta_{n s}=0.032$ the prediction for the tensor-to-scalar ratio is $r \approx 0.128$, which is larger than favored by the Planck data.

Márián et al. [118] have recently considered Psudo periodic Higgs inflation with a potential

$$
V=(1 / 2) m^{2} \hat{\phi}^{2}+V_{0}[1-\cos (\widetilde{\phi})]
$$

and shown that it corresponds well with the Planck data for a suitable value of $V_{0} / \mathrm{m}^{2}$.

\subsection{Hybrid Natural Inflation}

A potential problem for the original natural inflation model is that to produce sufficient inflation, the scale $f$ of symmetry breaking must be larger than the Planck energy, and we have no accepted theory to describe such conditions where quantum gravitation effects are expected to appear.

In order to cure this potential problem, and also to provide an inflationary model with one more free parameter than the natural inflation model, thereby increasing the possibility of obtaining a model in agreement with observations, a so-called hybrid natural inflation model has been introduced. In this model the inflaton field is supplied by a second field, which is responsible for terminating inflation. This model allows for a symmetry breaking scale that is less that the Planck scale. It is also assumed that the symmetry breaking energy is not greater than the Planck energy, meaning that $b \geq 1$.

Recent investigations of hybrid natural inflation have been performed by Ross and Germán [119], Carrillo-González et al. [58], Hebecker et al. [120], Vázquez et al. [121], Ross et al. [122], and G. Germán et al. [8]. For this model the inflaton potential is written as

$$
V(\phi)=V_{0}(1+a \cos \widetilde{\phi}) .
$$

where $a$ is a constant with a value either $-1 \leq a<0$ or $0<a \leq 1$. Here $a= \pm 1$ represents the original natural inflation. The slow roll parameters of the hybrid natural inflation model are

$$
\varepsilon=\frac{a^{2} b}{2} \frac{1-c_{\phi}^{2}}{\left(1+a c_{\phi}\right)^{2}}, \quad \eta=-a b \frac{c_{\phi}}{1+a c_{\phi}}, \quad \xi=-a^{2} b^{2} \frac{1-c_{\phi}^{2}}{\left(1+a c_{\phi}\right)^{2}}=-2 b \varepsilon
$$

where $b$ and $c_{\phi}$ is defined in Equation (6.5.3). It may be noted that the relationship (6.5.6) is valid in hybrid natural inflation while (6.5.4) is not. The scalar spectral tilt and the tensor-to-scalar-ratio are

$$
\delta_{n s}=a b \frac{3 a+2 c_{\phi}-a c_{\phi}^{2}}{\left(1+a c_{\phi}\right)^{2}} \quad, \quad r=8 a^{2} b \frac{1-c_{\phi}^{2}}{\left(1+a c_{\phi}\right)^{2}} .
$$

It follows from Equations (4.29), (6.6.2), and (6.6.3) that the running of the scalar spectral index is Carrillo-Gonzalez et al. [58] and Germán et al. [8],

$$
\alpha_{s}=\frac{r}{32}\left(3 r-16 \delta_{n s}+8 b\right) \text {. }
$$

In order to avoid quantum corrections of the potential for which we have no reliable theory, Ross and Germán [123] have considered the case that $M<M_{P}$, i.e., $b>1$. Then it follows from the 
first Equation (6.6.3) that in order to obtain a small $\delta_{n s}$, the parameter $a$ must be small. Hence we can use the approximation

$$
\delta_{n s} \approx 2 a b c_{\phi} .
$$

This shows that with $c_{\phi} \approx 1$ (see below) we must have $a<0.016$.

Hebecker et al. [124] have taken the initial value of the inflaton field to $\phi=(\pi / 2) M$ in order to maximize $r=16 \varepsilon$, giving $c_{\phi}=0$ and $r_{\mathrm{m}}=8 \beta^{2} b$. However at this point the potential is maximally steep, so this may be said to represent "fast roll inflation" [107] rather than slow roll inflation. For slow roll inflation a more natural starting point is close to $\phi \approx 0$ where the potential is flat. This gives $c_{\phi}^{2} \approx 1$ and hence a value of $r$ very close to zero (Ross et al. [119,122,123]). Let us see how close to zero. From the expressions (6.6.3) we get

$$
r=\frac{8 a s_{\phi}^{2}}{2 c_{\phi}+a\left(3-c_{\phi}^{2}\right)} \delta_{n s} \approx 4 a \widetilde{\phi}^{2} \delta_{n s} .
$$

With $\phi<M_{P}$ we have $\widetilde{\phi}^{2}<b$. From Equation (6.6.5) with $c_{\phi}^{2} \approx 1$ we also have $2 a \approx \delta_{n s} / b$. Hence Equation (6.6.6) lead to the inequality

$$
r<2 \delta_{n s}^{2}
$$

Inserting $\delta_{n s}=0.032$ gives $r<0.002$.

The initial value of the inflaton field can be determined in the usual way by using that the final value of the inflaton field is given by $\varepsilon\left(\phi_{f}\right)=1$ and the requirement that the slow roll era produces $N$ e-folds. This can be calculated analytically, but the expressions are not nice. We shall therefore follow a similar procedure as that of Hebecker et al. [124], and find the initial value of the inflaton field from $d r / d \phi=0$. This gives $c_{\phi}=\beta$ leading to

$$
r_{\max }=\frac{8 a^{2} b}{1-a^{2}} .
$$

Hebecker et al. have used $a=-0.1$. With Equation (6.6.1) this corresponds to $\phi=(\pi / 2.14) M$ close to the value chosen by Hebecker et al. The slow roll parameters and the spectral parameters are evaluated with this value of the inflaton field to first order in $c_{\phi}$ and $a$ (Ross et al. [122]). This gives

$$
\varepsilon \approx \frac{a^{2} b}{2}, \eta=-a b c_{\phi}, \quad \xi=-2 b \varepsilon,
$$

and

$$
\delta_{n s} \approx-2 \eta, \quad r \approx 8 a^{2} b, \quad \alpha_{S} \approx 2 a^{2} b^{2} .
$$

and hence,

$$
\alpha_{S} \approx(1 / 4) b r .
$$

With the values $a=-0.1, b=1, r=0.05$, we obtain $\alpha_{S} \approx 0.0125$ which is a somewhat larger than the values preferred by the Planck 2015 results.

Equation (6.6.4) can be written

$$
b=2 \delta_{n s}-\frac{3}{8} r+4 \frac{\alpha_{S}}{r} .
$$

This expression is valid both for the original natural inflation model and the hybrid natural inflation model. But the expression (6.5.9) for $\alpha_{S}$ leading to Equation (6.5.23) for $b$, is only valid for the original natural inflation model and is now replaced by the expression (6.6.12). With the BICEP2/Planck values $\delta_{n s}=0.032, r=0.05, \alpha_{S}=-0.003$ we get a negative value for $b$ which is not allowable. But there is a great uncertainty in the value of $\alpha_{S}$, namely $\alpha_{S}=0.003 \pm 0.007$. 
This encompasses $\alpha_{S}=0$, so the observational data permit a value of $\alpha_{S}$ close to zero, giving a positive value of $b$. The BICEP2/Planck data then give $b=0.02$ corresponding again to a symmetry breaking energy larger than the Planck energy. Values $b \geq 1$ corresponding to $M<M_{P}$ require approximately $\alpha_{s}>r / 4$. Hence taking account of the the most recent observational results, also the hybrid natural inflation models seems to be in trouble unless $\alpha_{S}>0$ and $r<4 \alpha_{s}$.

\subsection{Higgs Inflation}

Higgs inflation has recently been considered by Bezrukov et al. [125,126], by Gorbunov and Tokareva [127] and by Zeynizadeh and Akbarieh [128] in connection with observations of spectral properties of the cosmic microwave background radiation. Rubio [129] has given the Higgs potential as

$$
V(\phi)=\left\{\begin{array}{ll}
\left(V_{0} \xi^{2} / M_{P}^{4}\right)\left(\hat{\phi}^{2}-v^{2}\right)^{2}, & \hat{\phi}<<1 / \xi \\
V_{0}\left(1-e^{-\sqrt{2 / 3} \hat{\phi}}\right)^{2}, & \hat{\phi}>>1 / \xi
\end{array},\right.
$$

where $\xi$ is a dimensionless coupling constant with value $\xi \sim O\left(10^{4}\right)$, and $v$ is the vacuum expectation value of $\hat{\phi}$. The small field case has been discussed in Section 5.3 .

Usually Higgs inflation is concerned with the large field potential in the second line of Equation (6.7.1). It may be noted that the potential of the Starobinsky model has the same form [97,130-132]. Multiplying by $\left(1+e^{-\sqrt{2 / 3} \hat{\phi}}\right)^{2} /\left(1+e^{-\sqrt{2 / 3} \hat{\phi}}\right)^{2}$ and neglecting the term the $e^{-2 \sqrt{2 / 3} \hat{\phi}}$ in the numerator, the Higgs potential is often approximated by

$$
V=V_{0}\left(1+e^{-\sqrt{2 / 3} \hat{\phi}}\right)^{-2}
$$

Differentiation of the potential (4.7.2) gives

$$
V^{\prime}=\frac{4 M_{P} V_{0}}{\xi \sqrt{6}} \frac{h^{2}-M_{p}^{2} / \xi}{h^{4}} \quad, \quad V^{\prime \prime}=-\frac{4 V_{0}}{3 \xi} \frac{h^{2}-2 M_{p}^{2} / \xi}{h^{4}} \quad, \quad V^{\prime \prime \prime}=\frac{8 V_{0}}{3 \sqrt{6} \xi M_{P}} \frac{h^{2}-4 M_{p}^{2} / \xi}{h^{4}},
$$

where $h=\left(M_{P} / \sqrt{\xi}\right) \exp (\hat{\phi} / \sqrt{6})$. In the case of Higgs inflation one usually assumes that $h>>M_{P} / \sqrt{\xi}$. Hence $\left(M_{P}^{2} / \xi\right) / h^{2}<<1$. One therefore approximates the derivatives of the potential by

$$
V^{\prime}=\frac{4 M_{P} V_{0}}{\xi \sqrt{6}} \frac{1}{h^{2}}, \quad V^{\prime \prime} \approx-\frac{4}{3} \frac{V_{0}}{\xi} \frac{1}{h^{2}} \quad, \quad V^{\prime \prime \prime} \approx \frac{8}{3 \sqrt{6}} \frac{V_{0}}{\xi M_{P}} \frac{1}{h^{2}},
$$

We then obtain

$$
\varepsilon=\frac{4 M_{P}^{4}}{3 \xi^{2}} \frac{1}{h^{4}} \quad, \quad \eta=-\frac{4 M_{P}^{2}}{3 \xi} \frac{1}{h^{2}} \quad, \quad \xi=\frac{16 M_{P}^{4}}{9 \xi^{2}} \frac{1}{h^{4}}
$$

Hence

$$
\eta=-(2 \sqrt{\varepsilon / 3}) \quad, \quad \xi=(4 / 3) \varepsilon=\eta^{2}
$$

Using the same approximations in the calculation of the number of e-folds we have

$$
N \approx-\frac{1}{M_{P}^{2}} \int_{\phi}^{\phi_{f}} \frac{V_{0}}{V^{\prime}} d \phi \approx \frac{\xi \sqrt{6}}{4 M_{P}^{3}} \int_{\phi_{f}}^{\phi} e^{\sqrt{2 / 3}} \hat{\phi} d \phi \approx \frac{3}{4} \frac{h^{2}-h_{f}^{2}}{M_{P}^{2} / \xi} .
$$

The field strength $\phi_{f}$ at the end of the inflationary era is defined by $\varepsilon\left(\phi_{f}\right)=1$, giving

$$
h_{f}^{2}=\frac{2 M_{P}^{2}}{\xi \sqrt{3}}
$$


Inserting this into Equation (6.7.7) gives

$$
h^{2}=\frac{2 M_{P}^{2}}{3 \xi}(2 N+\sqrt{3}) .
$$

Equation (6.7.5) then gives

$$
\varepsilon=\frac{3}{(2 N+\sqrt{3})^{2}} \quad, \quad \eta=-\frac{2}{2 N+\sqrt{3}} \quad, \quad \xi=\left(\frac{2}{2 N+\sqrt{3}}\right)^{2},
$$

and Equations (4.13) and (4.4) then lead to

$$
\delta_{n s}=\frac{8 N+18+4 \sqrt{3}}{(2 N+\sqrt{3})^{2}}, \quad r=\frac{48}{(2 N+\sqrt{3})^{2}} .
$$

Neglecting the numbers of order unity compared to $N$ we get

$$
\varepsilon \approx \frac{3}{4 N^{2}}, \quad \eta \approx-\frac{1}{N} \quad, \quad \xi=\frac{1}{N^{2}},
$$

Equations (4.13), (4.15), (4.4), (4.29) and (4.43) now give

$$
\delta_{n s} \approx \frac{2}{N}, \quad n_{T} \approx-\frac{3}{2 N^{2}}, \quad r \approx \frac{12}{N^{2}}, \quad \alpha_{S} \approx-\frac{2}{N^{2}}, \quad \alpha_{T}=-\frac{3}{N^{3}} .
$$

Hence, with this approximation we have the relationships

$$
\begin{aligned}
& N=2 / \delta_{n s}, \quad r=3 \delta_{n s}^{2}, \quad \alpha_{S}=-(1 / 2) \delta_{n s}^{2}, \\
& n_{T}=-(3 / 8) \delta_{n s}^{2}=-r / 8, \quad \alpha_{T}=-(3 / 8) \delta_{n s}^{3}
\end{aligned}
$$

Inserting $\delta_{n s}=0.032$ gives $N=62, r=0.003, \alpha_{S}=0005, n_{T}=-0.0004, \alpha_{T}=-00001$. These are the predictions of the Higgs inflationary models, given the Planck 2015 value of $n_{\mathcal{S}}$. So far these values are not in conflict with any observational results. The BICEP2/Planck results seem to favor the Higgs inflationary models.

Lyth and Riotto [22] and later Drees et al. [101] and Sebastiani et al. [130] have investigated several inflationary models with similar potentials as the one in Equation (6.7.1), for example

$$
V(\phi)=V_{0}\left(1-e^{-q \hat{\phi}}\right) .
$$

They assumed that the dimensionless number $q$ is of order 1 . With the same approximations as for the Higgs potential and assuming that $e^{-q \hat{\phi}}<<1$ we then find

$$
\varepsilon=\left(q^{2} / 2\right) e^{-2 q \hat{\phi}}, \quad \eta=-q^{2} e^{-q \hat{\phi}}, \quad \xi=q^{4} e^{-2 q \hat{\phi}},
$$

giving

$$
\delta_{n s}=2 q^{2} e^{-q \hat{\phi}} \quad, \quad r=8 q^{2} e^{-2 q \hat{\phi}} \quad, \quad \alpha_{s}=-2 q^{4} e^{-2 q \hat{\phi}} .
$$

The number of e-folds is

$$
N=\left(1 / q^{2}\right)\left(e^{q \hat{\phi}}-e^{q \hat{\phi}_{f}}\right) .
$$

The value of the inflaton field as given by $\varepsilon\left(\phi_{f}\right)=1$ is given by

$$
e^{q \hat{\phi}_{f}}=q / \sqrt{2}
$$


Hence the value of the inflaton field during the slow roll era is given by

$$
e^{q \hat{\phi}}=q^{2} N+q / \sqrt{2} \approx q^{2} N .
$$

Inserting this into Equation (6.7.15) gives

$$
\delta_{n s} \approx \frac{2}{N} \quad, \quad r \approx \frac{8}{q^{2} N^{2}} \quad, \quad \alpha_{S} \approx-\frac{2}{N^{2}} .
$$

This leads to the consistency conditions

$$
r \approx\left(2 / q^{2}\right) \delta_{n s}^{2} \quad, \quad \alpha_{S} \approx-(1 / 2) \delta_{n s}^{2} .
$$

For this model the Planck/BICEP2 data with $q=1$ give $r=0.002, \alpha_{S}=0.0005$. It follows from Equation (4.54) and (6.7.22) that for this class of inflationary models the running of the tensor-to-scalar ratio is

$$
\hat{\alpha}_{r}=\left(1-\frac{1}{4 q^{2}}\right) \delta_{n s}
$$

\subsection{S-Dual Inflation}

This is a scenario [133] inspired by string theory. But like many other inflationary universe models it has a certain ad hoc character with some free parameters that can be adjusted so that the model cannot easily be falsified. Nevertheless the model has some nice mathematical properties. The potential is given by

$$
V(\phi)=\frac{V_{0}}{\cosh \widetilde{\phi}^{\prime}}
$$

where $\widetilde{\phi}=\phi / M$, and $V_{0}$ is a free parameter. Here the mass $M$ characterizes the energy scale where the inflation begins. An inflationary model with this potential will be considered in Section 6.20 as an example of tachyon inflationary models. It has recently been studied by Agarwal et al. [134].

We shall here consider the more general class of potentials

$$
V=V_{0} \cosh ^{p} \widetilde{\phi}
$$

where $p$ is a real number. With $p=1$ we have the potential

$$
V=V_{0} \cosh \widetilde{\phi}
$$

which has been studied by Bamba et al. [65].

Calculating the slow roll parameters from the expressions (3.1) with the potential (6.8.2) gives

$$
\begin{aligned}
& \varepsilon=(1 / 2) b p^{2} \tanh ^{2} \widetilde{\phi}, \quad \eta=b p\left[1+(p-1) \tanh ^{2} \widetilde{\phi}\right], \\
& \xi=b^{2} p^{2}\left[3 p-2+(p-1)(p-2) \tanh ^{2} \widetilde{\phi}\right] \tanh ^{2} \widetilde{\phi} .
\end{aligned}
$$

where $b$ is given in Equation (6.5.3). Since $\tanh ^{2} \hat{\phi}<1$ we have that $\varepsilon<\left(p^{2} / 2\right) b$. Hence, it is clear that this class of inflationary universe models must be completed by adding a mechanism making it possible to have a "graceful exit" from the inflationary era.

Inserting the expressions (6.8.4) into Equations (4.13), (4.15), (4.4), (4.29) and (4.43) we find that the spectral indices of the scalar fluctuations, its running and the tensor-scalar ratio are

$$
\begin{aligned}
& \delta_{n S}=b p\left[(p+2) \tanh ^{2} \widetilde{\phi}-2\right], \quad n_{T}=-b p^{2} \tanh ^{2} \widetilde{\phi}, \quad r=8 b p^{2} \tanh ^{2} \widetilde{\phi}, \\
& \alpha_{S}=2 b^{2} p^{2}(p+2) \frac{\tanh ^{2} \widetilde{\phi}}{\cosh ^{2} \widetilde{\phi}}, \quad \alpha_{T}=2 b^{2} p^{3} \frac{\tanh ^{2} \widetilde{\phi}}{\cosh ^{2} \widetilde{\phi}} .
\end{aligned}
$$


The expression for $\delta_{n s}$ may be written

$$
\delta_{n s}=b p \frac{p \sinh ^{2} \widetilde{\phi}-2}{\cosh ^{2} \widetilde{\phi}}
$$

Hence $\delta_{n s}>0$ requires either $p \sinh ^{2} \widetilde{\phi}<2$ or $p<0$. Note also that

$$
\alpha_{r}=\frac{p}{2+p} \alpha_{s}
$$

Defining the end of inflation by $\varepsilon\left(\phi_{f}\right)=1$ we have

$$
b p^{2} \tanh ^{2} \phi_{f}=2 .
$$

Since $\tanh ^{2} \phi_{f}<1$ Equation (6.8.8) leads to the requirement $b>2 / p^{2}$ or $M<(|p| / \sqrt{2}) M_{p}$. Integrating Equation (3.50) with the expression for $\varepsilon$ in Equation (6.8.4) the number of e-folds is

$$
N=\frac{1}{b p} \ln \frac{\sinh \widetilde{\phi}}{\sinh \widetilde{\phi}_{f}}
$$

in agreement with Equation (6) of Anchordoqui et al. [133]. They have argued from this equation that the requirement that there is roughly 50-60 e-folds of expansion during the inflationary era implies that $M>M_{P}$, i.e., that $b<1$. It follows that the standard condition $\varepsilon\left(\phi_{f}\right)=1$ for the final value of the inflaton field, and a graceful exit of the slow roll era, cannot be fulfilled in this class of inflationary models.

Note that $\widetilde{\phi}<\widetilde{\phi}_{f}$ if $p<0$. The factor $1 / b$ is the xpression for $N$ is lacking in Equation (III.14) of Bamba et al. [65]. It follows from Equation (6.8.9) that

$$
\sinh \widetilde{\phi}=\sinh \widetilde{\phi}_{f} e^{b p N} .
$$

Inserting this equation into the first of the Equation (6.8.5) leads to

$$
\sinh ^{2} \widetilde{\phi}_{f}=\frac{2 b p+\delta_{n s}}{b p^{2}-\delta_{n s}} e^{-2 b p N}
$$

which requires $b>\delta_{n s} / p^{2}$. The last two equations give

$$
\sinh ^{2} \widetilde{\phi}=\frac{2 b p+\delta_{n s}}{p^{2} b-\delta_{n s}}
$$

Hence the inequality $p \sinh ^{2} \widetilde{\phi}>2$ requires $p>-2$.

In their first example Bamba et al. have used the values $N=50$ and $\hat{\phi}_{f} \approx 10^{-20}$ that represent the small field regime. This leads to $\tanh \widetilde{\phi}_{f} \approx \sinh \widetilde{\phi}_{f} \approx \widetilde{\phi}_{f}$ and $\cosh \hat{\phi}_{f} \approx 1$. Hence $\varepsilon \approx\left(p^{2} / 2\right) b \widetilde{\phi}_{f}^{2}$ and $\eta \approx p(2 p-1) b$ giving $\varepsilon \approx[p / 2(2 p-1)] \hat{\phi}_{f}^{2} \eta<<\eta$ and

$$
\delta_{n s} \approx-2 \eta \approx 2 p(1-2 p) b,
$$

giving

$$
p \approx \frac{1}{4}\left(1 \pm \sqrt{1-4 \frac{\delta_{n s}}{b}}\right)>0
$$

in conflict with the Planck data. 
It follows from the first and third of the Equation (6.8.5) that the $r, \delta_{n s}-$ relationship for this class of inflationary universe models has the form,

$$
r=\frac{8 p}{p+2}\left(\delta_{n s}+2 b p\right)
$$

Solving this with respect to $b$ gives

$$
b=\frac{(p+2) r-8 p \delta_{n s}}{16 p^{2}}
$$

which requires

$$
p<\frac{2 r}{8 \delta_{n s}-r}
$$

For $\delta_{n s}=0.032$ and $r<0.04$ this gives the condition $p<0.37$. The value $p=1$, for example, is not allowed by the PBK-data. The model with $p=-1$ must have $b<0.0185$, i.e., $M>7.35 M_{p}$.

\subsection{Hyperbolic Inflation}

Basilakos and Barrow [135] have considered a class of models of inflation that is very similar to S-dual inflation. They have called it hyperbolic inflation.

We shall here consider a flat universe model in this class with radiation and inflaton energy. In this model the inflaton field has the potential

$$
V(\phi)=A \sinh ^{p} \widetilde{\phi}
$$

where

$$
A=(3 / 2)(1-w) \Omega^{1-p / 2}(1-\Omega)^{p / 2} H_{0}^{2}, \quad p=\frac{6(1+w)}{1-3 w},
$$

and $w$ is the equation of state parameter of the inflaton energy and $\Omega$ its mass density parameter. Furthermore $\widetilde{\phi}=\phi / M$, where $M$ represents the energy scale during the slow roll era. The potential (6.9.1) leads to the following expressions for the slow roll parameters

$$
\begin{aligned}
& \varepsilon=(b / 2) p^{2} \operatorname{coth}^{2} \widetilde{\phi}, \quad \eta=b p\left[1+(p-1) \operatorname{coth}^{2} \widetilde{\phi}\right] . \\
& \xi=b^{2} p^{2}\left[3 p-2+(p-1)(p-2) \operatorname{coth}^{2} \widetilde{\phi}\right] \operatorname{coth}^{2} \widetilde{\phi}
\end{aligned} .
$$

where $b=M_{P}^{2} / M^{2}$. Since $|\operatorname{coth} \widetilde{\phi}|>1$ this class of inflationary models does not have the exit problem of the S-dual models. It follows from the expression (6.9.3) that

$$
\eta=\frac{2(p-1)}{p} \varepsilon+b p^{2}, \quad \xi=\left[b(3 p-2)+\frac{2(p-1)(p-2)}{p^{2}} \varepsilon\right] 2 \varepsilon .
$$

Hence the spectral parameters of the scalar fluctuations are

$$
\delta_{n s}=b p\left[(p+2) \operatorname{coth}^{2} \widetilde{\phi}-2\right] \quad, \quad r=8 b p^{2} \operatorname{coth}^{2} \widetilde{\phi}
$$

The $\delta_{n s}, r$-relation is

$$
r=\frac{8 p}{2+p}\left[\delta_{n s}+2 b p\right] .
$$

Solving this equation with respect to $M$ we can estimate the energy scale where the inflation begins in these models from the Planck and BICEP2 data,

$$
M=\frac{4 p}{\sqrt{(2+p) r-8 p \delta_{n s}}} M_{P}
$$


With for example $p=0.10, \delta_{n s}=0.032, r=0.04$ Equation (6.9.7) gives $M \approx 1.7 M_{P}$ or $b=0.35$.

In this class of inflationary models the number of e-folds is

$$
N=\frac{1}{b p} \ln \frac{\cosh \widetilde{\phi}}{\cosh \widetilde{\phi}_{f}}
$$

If the logarithmic factor is of order one, this requires that $b$ is of the order $b \simeq 1 / p N$ and hence $M \simeq \sqrt{p N} M_{P}$. For say $p>0.1$ this gives $M>2.3 M_{P}$. Hence in order to be compatible with the Planck/BICEP2 results the energy scale of this model must be larger than the Planck energy.

The value of the inflaton field at the end of the inflationary era is defined by $\varepsilon\left(\widetilde{\phi}_{f}\right)=1$, giving

$$
\operatorname{coth}^{2} \widetilde{\phi}_{f}=\frac{2}{b p^{2}}
$$

Hence there is a graceful exit of the inflationary era only if $b<2 / p^{2}$ or $M>(p / \sqrt{2}) M_{P}$ which is fulfilled in the case of large field inflation. Inserting Equation (6.9.9) into Equation (6.9.8) gives

$$
\cosh ^{2} \widetilde{\phi}=\frac{e^{2 b p N}}{1-(b / 2) p^{2}}
$$

Inserting this into Equation (6.9.5) gives

$$
\delta_{n s}=b p \frac{p+\left(2-b p^{2}\right) e^{-2 b p N}}{1+\left[(b / 2) p^{2}-1\right] e^{-2 b p N}} \quad, \quad r=\frac{8 b p^{2}}{1+\left[(b / 2) p^{2}-1\right] e^{-2 b p N}}
$$

\subsection{M-Flation}

Another string theory motivated inflation model is called M-flation [39]. In this model the potential is

$$
V(\phi) \propto \phi^{2}(\phi-\mu)^{2}
$$

where $\mu>M_{P}$ represent the energy per particle necessary to initiate inflation. The slow roll parameters are

$$
\varepsilon=\frac{2}{\kappa} \frac{(2 \phi-\mu)^{2}}{\phi^{2}(\phi-\mu)^{2}} \quad, \quad \eta=\frac{1}{\kappa} \frac{3(2 \phi-\mu)^{2}-\mu^{2}}{\phi^{2}(\phi-\mu)^{2}}
$$

This leads to

$$
\delta_{n s}=\frac{2}{\kappa} \frac{3(2 \phi-\mu)^{2}+\mu^{2}}{\phi^{2}(\phi-\mu)^{2}} \quad, \quad r=-8 n_{T}=\frac{32}{\kappa} \frac{(2 \phi-\mu)^{2}}{\phi^{2}(\phi-\mu)^{2}} .
$$

The slow roll period ends when $\varepsilon=1$. Inserting this into the first of the Equation (6.10.2) leads to a fourth degree equation for $\phi_{f}$. This can be rewritten as a second degree equation by introducing a new quantity representing the inflaton field, $y \equiv \phi(\phi-\mu)$, i.e., $(2 \phi-\mu)^{2}=4 y+\mu^{2}$. Considering the region $\phi>\mu$ we have $y>0$. Then the solution of the equation for $\phi_{f}$ is

$$
y_{f}=4 M_{P}^{2}(1+\sqrt{1+x / 8}) \quad, \quad x=\left(\mu / M_{P}\right)^{2} .
$$

Expressing the number of e-folds in terms of $y$ we obtain

$$
N=\kappa \int_{\phi_{f}}^{\phi} \frac{V}{V^{\prime}} d \phi=\frac{\kappa}{8}\left(y-y_{f}+\frac{\mu^{2}}{4} \ln \frac{4 y+\mu^{2}}{4 y_{f}+\mu^{2}}\right)
$$


It follows from Equation (6.10.3) that

$$
r=\frac{16}{3}\left(\delta_{n s}-\frac{2}{\kappa} \frac{\mu^{2}}{y^{2}}\right)
$$

Hence, a prediction of the M-flation model is

$$
r<\frac{16}{3} \delta_{n s}
$$

With the Planck value $\delta_{n s}=0.032$ this gives $r<0.17$. In terms of $y$ the expression (6.10.3) for $\delta_{n s}$ takes the form

$$
\delta_{n s}=8 M_{P}^{2} \frac{3 y+\mu^{2}}{y^{2}} .
$$

Solving this equation with respect to $y$ we get

$$
y=\frac{12 M_{P}^{2}}{\delta_{n s}}\left(1+\sqrt{1+\frac{1}{18} \delta_{n s} x}\right) .
$$

Inserting this into Equation (6.10.6) we obtain

$$
r=\frac{16}{3} \delta_{n s}\left[1-\frac{(1 / 72) \delta_{n s} x}{\left(1+\sqrt{1+(1 / 18) \delta_{n s} x}\right)^{2}}\right] .
$$

The function (6.10.10) is plotted in Figure 7 for $\delta_{n s}=0.032$.

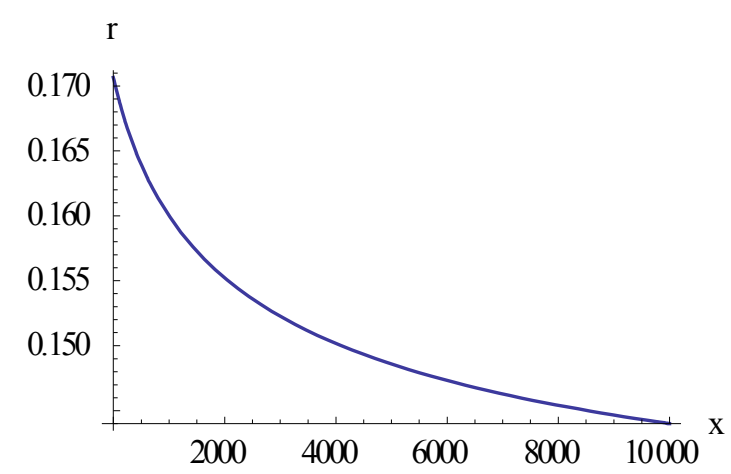

Figure 7. The predicted values of the tensor-to-scalar ratio $r$ by the M-flation model are here shown by plotting $r$ as a function of $x=\mu^{2} / M_{P}^{2}$ for $0<\mu<100 M_{P}$.

The expression (6.10.10) gives $\lim _{x \rightarrow \infty} r=4 \delta_{n s}$. Hence with the Planck value of $\delta_{n s}$ this inflationary model predicts that $r>0.128$ which is larger than the values allowed by the BICEP2/Planck 2015 result.

\subsection{Supergravity Motivated Inflation}

Kallosh et al. $[79,132,136]$ have studied a class of inflationary models motivated from supergravity, which they call $\alpha-$ attractor models. One version has potential

$$
V(\phi)=V_{0} \alpha^{p / 2} \tanh ^{p} \widetilde{\phi}, M=M_{P} \sqrt{6 \alpha},
$$


where $\widetilde{\phi}=\phi / M, p$ is an arbitrary constant, and $M$ represents the characteristic energy per particle at the beginning of the inflationary era. The parameter $\alpha$ can take any positive value. With the inflaton potential (6.11.1) the spectral index $n_{S}$ and the tensor-to-scalar ratio are given by

$$
\delta_{n s}=4 b p \frac{p+2 \cosh (2 \widetilde{\phi})}{\sinh ^{2}(2 \widetilde{\phi})} \quad, \quad r=\frac{32 b p^{2}}{\sinh ^{2}(2 \widetilde{\phi})}
$$

where $b=M_{P}^{2} / M^{2}$, and $\phi=\phi(N)$ is the inflaton field at the beginning of the slow roll era, $N$ e-folds before it ends. Gong and Shin [137] have considered models with $p=2$ in this class of models and called them 'natural cliff inflation'. In this case the expressions (6.11.2) lead to the $\delta_{n s}, r$ - relationship

$$
r=\frac{4 \delta_{n s}}{1+8 b / \delta_{n s}} .
$$

For the Planck 2015 value $\delta_{n s}=0.032$ this gives $r<4 \delta_{n s}=0.128$. Equation (6.3.11) may be written

$$
b=\frac{\delta_{n s}}{8}\left(4 \frac{\delta_{n s}}{r}-1\right) .
$$

Inserting $\delta_{n s}=0.032, r=0.04$ gives $b=0.009$ or $M \approx 10 M_{P}$.

The number of e-folds is

$$
N=\kappa \int_{\phi_{f}}^{\phi} \frac{V}{V^{\prime}} d \phi=\frac{1}{4 b p}\left[\cosh (2 \widetilde{\phi})-\cosh \left(2 \widetilde{\phi}_{f}\right)\right] .
$$

The end of this era is determined by $\varepsilon\left(\phi_{f}\right)=1$. This gives

$$
\sinh \left(2 \widetilde{\phi}_{f}\right)=\sqrt{2 b} p .
$$

Inserting Equations (6.11.4) and (6.11.5) into the expressions (6.11.2) leads to

$$
\begin{aligned}
& \delta_{n s}=\frac{2 N+(3 / 2) \alpha+(1 / p) \sqrt{3 \alpha\left(3 \alpha+p^{2}\right)}}{N^{2}+(N / p) \sqrt{3 \alpha\left(3 \alpha+p^{2}\right)}+(3 / 4) \alpha}, \\
& r=\frac{12 \alpha}{N^{2}+(N / p) \sqrt{3 \alpha\left(3 \alpha+p^{2}\right)}+(3 / 4) \alpha} .
\end{aligned}
$$

For $\alpha<<N$ and $p<<N$ the expressions (4.11.6) reduce to

$$
\delta_{n s}=\frac{2}{N}, \quad r=\frac{12 \alpha}{N^{2}} .
$$

In this case the model allows small values of $r$ in agreement with BPK-results. For $\alpha>>N^{2}$ and $\alpha>>p^{2}$ we get

$$
\delta_{n s} \approx 2 \frac{p+2}{p+4 N} \quad, \quad r=\frac{16 p}{p+4 N} .
$$

These are the same predictions as those of chaotic inflation in Equation (6.1.36), and are ruled out by the BICEP2/Planck 2015 results.

The $\alpha$ - inflation models have more recently been discussed by Kallosh and Linde [138]. Among others they considered a model where the inflaton field has a potential (6.1.11) with $p=2$. They also considered an $\alpha$ - attractor model with a potential similar to the one in Equation (6.7.1) for the Higgs inflation, namely

$$
V(\phi)=V_{0}\left(1-e^{-\sqrt{2 / 3 \alpha} \hat{\phi}}\right)^{2} .
$$


In this case and making the approximation that we can put $V \approx V_{0}$ in the numerators of the expressions for the slow roll parameters, the spectral parameters $n_{S}$ and $r$ are

$$
\delta_{n s}=\frac{8 N+18 \alpha+4 \sqrt{3 \alpha}}{(2 N+\sqrt{3 \alpha})^{2}}, \quad r=\frac{48 \alpha}{(2 N+\sqrt{3 \alpha})^{2}} .
$$

which reduces to Equation (6.7.11) for $\alpha=1$. These expressions give

$$
r=\frac{24 \alpha}{4 N+9 \alpha+2 \sqrt{3 \alpha}} \delta_{n s}
$$

Inserting $N=50, r=0.04, \delta_{n s}=0.032$ gives $\alpha=22.4$. However, the mentioned approximation is only valid when $\sqrt{2 / 3 \alpha}\left(\phi / M_{P}\right)>>1$, i.e., when $\alpha<<(2 / 3)\left(\phi / M_{P}\right)^{2}$. Hence the expressions (6.11.10) are not valid for large values of $\alpha$.

Let us calculate the corresponding expressions valid for large values of $\alpha$. The slow roll parameters $\varepsilon$ and $\eta$ are

$$
\varepsilon=\frac{4}{3 \alpha} \frac{1}{\left(e^{\sqrt{2 / 3 \alpha}}-1\right)^{2}} \quad, \quad \eta=-\frac{4}{3 \alpha} \frac{e^{\sqrt{2 / 3 \alpha}}-2}{\left(e^{\sqrt{2 / 3 \alpha}}-1\right)^{2}} .
$$

The spectral parameters $n_{S}$ and $r$ are

$$
\delta_{n s}=\frac{8}{3 \alpha} \frac{e^{\sqrt{2 / 3 \alpha} \phi}+1}{\left(e^{\sqrt{2 / 3 \alpha} \phi}-1\right)^{2}} \quad, \quad r=\frac{64}{3 \alpha} \frac{1}{\left(e^{\sqrt{2 / 3 \alpha} \phi}-1\right)^{2}} .
$$

These expressions lead to the following $\delta_{n s}, r-$ relation

$$
r=4 \delta_{n s}-(8 / 3 \alpha)\left(\sqrt{1+3 \alpha \delta_{n s}}-1\right) .
$$

This relationship is plotted as a function of $\alpha$ in Figure 8 for $1 \leq \alpha \leq 1000$ with $\delta_{n s}=0.032$. Note that $\lim _{\alpha \rightarrow \infty} r=4 \delta_{n s}=0.128$.

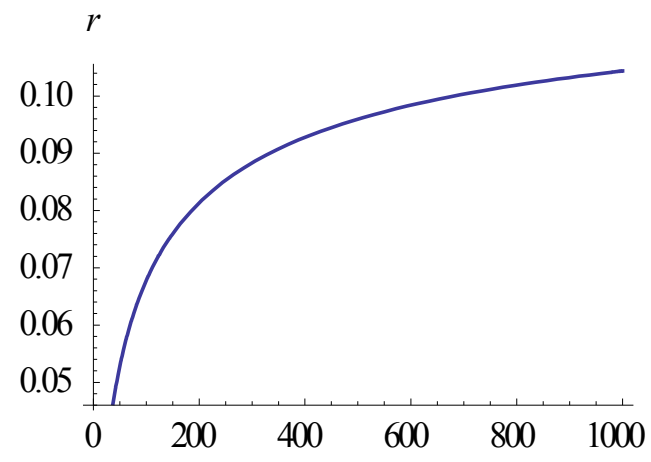

Figure 8. The relationship (6.11.14) plotted with $\delta_{n s}=0.032$.

The values of $r$ shown in this figure are allowed by the Planck/BICEP2 data.

\subsection{Goldstone Inflation}

Lyth and Riotto [22] write that the pseudo-Goldstone boson, coming from instanton effects, is typically of the form

$$
V(\phi)=V_{0} \cos ^{2} \hat{\phi},
$$


where $\hat{\phi}=\phi / M$ and $M$ is a mass significantly bigger than the Planck mass, $M_{P}$. With this potential the slow roll parameters are

$$
\varepsilon=2 b \tan ^{2} \hat{\phi}, \quad \eta=\varepsilon-2 b \quad, \quad b=\left(M_{P} / M\right)^{2} .
$$

The final value of the inflaton field at the end of the slow roll era is given by

$$
\tan ^{2} \hat{\phi}_{f}=1 / 2 b \text {. }
$$

The number of e-folds is

$$
N=\frac{1}{2 b} \ln \frac{\sin \hat{\phi}_{f}}{\sin \hat{\phi}},
$$

where $\phi$ is the value of the inflaton field at a point of time with $N$ e-folds before the end of the slow roll era. Inserting the expression (6.12.3) for $\phi_{f}$ into Equation (6.12.4) gives for the inflaton field at this point of time

$$
\tan ^{2} \hat{\phi}=\frac{1}{(1+2 b) e^{4 b N}-1} .
$$

Inserting this into the expressions (4.11.2) for $\varepsilon$ and $\eta$ and using Equations (3.4) and (3.13) give

$$
\delta_{n s}=4 b \frac{(1+2 b) e^{4 b N}+1}{(1+2 b) e^{4 b N}-1} \quad, \quad r=\frac{32 b}{(1+2 b) e^{4 b N}-1} .
$$

These expressions for $n_{S}$ and $r$ lead to

$$
b=(1 / 16)\left(4 \delta_{n s}-r\right) .
$$

The BICEP2/Planck values $\delta_{n s}=0.032$ and $r=0.05$ gives $b=0.005$ or $M \approx 14 M_{P}$, which means that this is a large field inflation model, possibly depending upon conditions outside the region of applicability of the general theory of relativity. It follows from Equations (4.29), (6.12.5) and (6.12.6) that

$$
\alpha_{S}=-\frac{r}{32}(r+32 b)=\frac{r}{32}\left(r-8 \delta_{n S}\right) .
$$

From Equation (3.46) we have

$$
\alpha_{r}=-4 \alpha_{s} .
$$

With the BICEP2/Planck values of $r$ and $\delta_{n s}$ this model gives $\alpha_{S}=-0.0003$ and $\alpha_{r}=0.0012$. Solving the last of the Equations (6.12.5) with respect to $N$ leads to

$$
N=\frac{1}{4 b} \ln \frac{r+32 b}{r(1+2 b)} .
$$

Inserting the values of $r$ and $b$ gives $N=72$.

It may be noted that this model is mathematically identical to one of the natural inflation models.

\subsection{Coleman-Weinberg Inflation}

The Coleman-Weinberg (CW) potential [139] has the form [105,140,141]

$$
V(\phi)=V_{0}\left\{\hat{\phi}^{4}\left[\ln \hat{\phi}-\frac{1}{4}\right]+\frac{1}{4}\right\},
$$

where $\hat{\phi}=\phi / M$. The shape of this potential is similar to that of the symmetry breaking potential in Figure 2, and $M$ is the value of the field where the potential has a minimum. We shall assume that this value of the field is much less than the Planck mass, $M<<M_{P}$. 
Two types of inflation are possible when the inflaton field has the CW potential: the large field inflation (LFI) and the small field inflation (SFI). The LFI is similar to polynomial inflation with $n=4$. We shall here take a closer look at the SFI branch of the CW inflation.

We then assume that $\phi<<M$. The derivatives of the potential (6.13.1) are

$$
V^{\prime}=\frac{4 V_{0}}{M} \hat{\phi}^{3} \ln \hat{\phi}, \quad V^{\prime \prime}=\frac{4 V_{0}}{M^{2}} \hat{\phi}^{2}(1+3 \ln \hat{\phi}) \quad, \quad V^{\prime \prime \prime}=\frac{4 V_{0}}{M^{3}} \hat{\phi}(5+6 \ln \hat{\phi}) .
$$

Since $|\ln (\phi / M)|=|\ln (M / \phi)|>>1$ we can approximate the second and third derivatives by

$$
V^{\prime \prime} \approx \frac{12 V_{0}}{M^{2}} \hat{\phi}^{2} \ln \hat{\phi}, \quad V^{\prime \prime \prime} \approx \frac{24 V_{0}}{M^{3}} \hat{\phi} \ln \hat{\phi} .
$$

Using these expressions together with the approximation $V \approx V_{0} / 4$ we obtain for the slow roll parameters

$$
\varepsilon=128 b \hat{\phi}^{6}(\ln \hat{\phi})^{2}, \quad \eta=48 b \hat{\phi}^{2} \ln \hat{\phi}, \quad \xi=16 \sqrt{6} b \hat{\phi}^{2} \ln \hat{\phi} .
$$

where $b=M_{P} / M$. Note that $\eta, \xi<0$ since $\phi<M$. We see that $\varepsilon<<|\eta|$. Hence we can approximate $n_{S}$ with

$$
\delta_{n s} \approx-2 \eta .
$$

The e-folding number is given by

$$
N=\frac{1}{M_{P}^{2}} \int_{\phi_{f}}^{\phi} \frac{V}{V^{\prime}} d \phi \approx \frac{\mu^{4}}{16 M_{P_{\phi_{f}}^{2}}^{2}} \int_{\phi^{3} \ln (\phi / M)}^{d \phi} .
$$

During slow roll the quantity $\ln (\phi / \mu)$ changes so slowly that one can approximate the integral by considering it as a constant. This gives

$$
N \approx-\frac{\mu^{4}}{32 M_{P}^{2}}\left[\frac{1}{\phi^{2} \ln \hat{\phi}}\right]_{\phi_{f}}^{\phi} .
$$

Using the expression for $\eta$ in Equation (6.13.4) we have

$$
N \approx \frac{3}{2}\left(\frac{1}{|\eta|}-\frac{1}{\left|\eta_{f}\right|}\right) .
$$

For this inflationary model the end of the slow roll era is given by $\left|\eta_{f}\right|=1$. Since $1 /|\eta|>>1$ we we can neglect the last term inside the parenthesis, and obtain

$$
\eta \approx-\frac{3}{2 N}
$$

Equations (6.13.5) and (6.13.9) gives

$$
\delta_{n s} \approx \frac{3}{2 N} .
$$

Inserting the Planck 2015 value of $n_{S}$ gives $N=47$. From the expressions (6.13.4) for $\varepsilon$ and $\eta$, and using Equation (6.13.5) together with Equation (4.4) we obtain

$$
r \approx \frac{27}{3456} \frac{1}{b N^{3}} \frac{1}{\ln \hat{\phi}}=\frac{\delta_{n s}^{3}}{432 b \ln \hat{\phi}} .
$$


Hence this inflationary model predicts that $r \approx 0$. Equations (3.21) and (6.13.11) lead to

$$
\alpha_{S}=-(2 / 3) \delta_{n S}^{2}
$$

giving $\alpha_{S}=-0.0007$.

\subsection{Kähler Moduli Inflation}

The Kähler moduli inflation was introduced by Conlon and Quevedo [142] and is characterized by a potential [70]

$$
V(\phi)=V_{0}\left(1-\alpha \hat{\phi}^{4 / 3} e^{-\beta \hat{\phi}^{4 / 3}}\right) .
$$

It is assumed that $\beta \hat{\phi}^{4 / 3}>>1$ and hence that $\alpha \hat{\phi}^{4 / 3} e^{-\beta \hat{\phi}^{4 / 3}}<<1$. We can therefore approximate the slow roll parameters $\varepsilon$ and $\eta$ by Equation (6.2.5). This gives

$$
\begin{aligned}
& \varepsilon=(8 / 9 \kappa) \alpha^{2} \hat{\phi}^{2 / 3} e^{-2 \beta \hat{\phi}^{4 / 3}}\left(\beta \hat{\phi}^{4 / 3}-1\right)^{2} \approx(8 / 9 \kappa) \alpha^{2} \beta^{2} \hat{\phi}^{-2 / 3}\left(\hat{\phi}^{2} e^{-\beta \hat{\phi}^{4 / 3}}\right)^{2} . \\
& \eta \approx(4 / 3) \alpha \beta\left[(5 / 3) \hat{\phi}^{2 / 3}-(4 / 3) \beta \hat{\phi}^{2}\right] e^{-\beta \hat{\phi}^{4 / 3}} \approx-(16 / 9) \alpha \beta^{2} \hat{\phi}^{2} e^{-\beta \hat{\phi}^{4 / 3}} .
\end{aligned}
$$

The number of e-folds during the slow roll era is

$$
N=\int_{\hat{\phi}_{f}}^{\hat{\phi}} \frac{1}{\sqrt{2 \kappa \varepsilon}} d \hat{\phi} \approx \frac{3}{4 \alpha \beta} \int_{\hat{\phi}_{f}}^{\hat{\phi}} \frac{e^{\beta \hat{\phi}^{4 / 3}}}{\hat{\phi}^{5 / 3}} d \hat{\phi} .
$$

Introducing a variable $x=\beta \hat{\phi}^{4 / 3}$ we find that for $\beta \hat{\phi}^{4 / 3}>>1$ this gives approximately

$$
N \approx \frac{9}{16 \alpha \beta^{2}}\left(\frac{e^{\beta \hat{\phi}^{4 / 3}}}{\hat{\phi}^{2}}-\frac{e^{\beta \hat{\phi}_{f}^{4 / 3}}}{\hat{\phi}_{f}^{2}}\right) .
$$

In this model the inflaton field is assumed to be much less at the end of the inflationary era than at the beginning, $\phi_{f}<<\phi$. Hence

$$
N \approx \frac{9}{16 \alpha \beta^{2}} \frac{e^{\beta \phi^{4 / 3}}}{\phi^{2}}
$$

giving

$$
\ln N=\beta \hat{\phi}^{4 / 3}+\ln \left(9 / 16 \alpha \beta^{2} \hat{\phi}^{2}\right) \approx \beta \hat{\phi}^{4 / 3} .
$$

Substituting from Equations (6.14.5) and (6.14.6) into Equation (6.14.2) gives

$$
\varepsilon(N) \approx \frac{9}{32 \beta^{3 / 2} N^{2} \sqrt{\ln N}}, \quad \eta(N) \approx-\frac{1}{N} .
$$

Hence $\varepsilon<<\eta$, so that

$$
\delta_{n s} \approx-2 \eta=\frac{2}{N} .
$$

With $\delta_{n s}=0.032$ this gives $N=62$. For $\beta \approx 1$ this gives a very small value of the tensor-to-scalar ratio,

$$
r \approx \frac{9}{2 N^{2} \sqrt{\ln N}} \approx 0.0003
$$

which is permitted by the BICEP2/Planck result. 
With a redefinition $\hat{\phi}^{4 / 3} \rightarrow \hat{\phi}$ the potential (6.14.1) takes the form

$$
V(\phi)=V_{0}\left(1-\alpha \hat{\phi} e^{-\beta \hat{\phi}}\right) .
$$

A similar model considered by Garcia-Bellido and Roest [70] has potential

$$
V=V_{0}\left(1-\alpha \hat{\phi} e^{\hat{\phi}}\right)
$$

with $\alpha \hat{\phi}>>1$. This form of the Kähler moduli inflation was also considered by Martin et al. [33]. Then the slow roll parameters are

$$
\varepsilon \approx(1 / 2) \alpha^{2} \hat{\phi}^{2} e^{-2 \hat{\phi}}, \eta \approx-\alpha \hat{\phi} e^{-\hat{\phi}} .
$$

The number of e-folds is

$$
N=\frac{1}{\alpha} \int_{\hat{\phi}_{f}}^{\hat{\phi}} \frac{e^{\hat{\phi}}}{\hat{\phi}} d \hat{\phi} \approx \frac{1}{\alpha} E i(\hat{\phi}),
$$

where $\operatorname{Ei}(\hat{\phi})$ is the exponential integral, and we have neglected the lower integration limit. This function has a series expansion for large $\phi$

$$
E i(\hat{\phi})=e^{\hat{\phi}}\left(\frac{1}{\hat{\phi}}+\frac{1}{\hat{\phi}^{2}}+\frac{2}{\hat{\phi}^{3}}+\cdots\right)
$$

Hence, we have approximately

$$
N \approx \frac{1}{\alpha} \frac{e^{\hat{\phi}}}{\hat{\phi}}
$$

Inserting this into Equation (6.14.2) leads to

$$
\varepsilon \approx \frac{1}{2 N^{2}}, \eta \approx-\frac{1}{N^{\prime}}
$$

giving

$$
\delta_{n s} \approx \frac{2}{N}, \quad r \approx \frac{8}{N^{2}}, \quad \alpha_{S}=-\frac{2}{N^{2}} .
$$

Again the Planck result $\delta_{n s}=0.032$ gives $N=62$. Hence $r=0.002$ and $\alpha_{S}=-0.0005$.

\subsection{Hybrid Inflation}

Hybrid inflation involves two fields, the so-called water fall field, $\chi$, and the inflaton field, $\phi$. The potential is given by [143]

$$
V(\chi, \phi)=g^{2}\left(M^{2}-\frac{\chi^{2}}{4}\right)^{2}+\frac{m^{2}}{2} \phi^{2}+\frac{\lambda^{2}}{4} \chi^{2} \phi^{2} .
$$

where $M, m$ are mass parameters, and $g, \lambda$ are dimensionless constants.

Rehman et al. [105] have revisited hybrid inflation in light of WMAP5 data. We shall here follow their exposition. The global minima of the potential lie at $(\chi, \phi)=( \pm 2 M, 0)$. For $\phi>\sqrt{2} g M / \lambda$ the only minimum of the potential lies at $\chi=0$. In this region the potential reduces to

$$
V(\phi)=V_{0}\left(1+\widetilde{\phi}^{2}\right) \quad, \quad \widetilde{\phi}=m \phi / \sqrt{2 V_{0}} \quad, \quad V_{0}=g^{2} M^{4} .
$$

Note the similarity of this potential with the Hilltop potential (6.2.1). The only essential difference is the sign inside the parenthesis. 
Slow roll inflation happens as the inflaton field $\phi$ rolls down the $\chi=0$ valley. Upon reaching the waterfall inflation ends abruptly as the inflaton field rapidly falls into one of the global minima. This scenario is termed "hybrid" because the vacuum energy density $V_{0}$ is provided by the waterfall field $\chi$, while $\phi$ is the slowly rolling inflaton field.

With the potential (6.15.2) the slow roll parameters are

$$
\varepsilon=\frac{\eta_{0} \widetilde{\phi}^{2}}{\left(1+\widetilde{\phi}^{2}\right)^{2}} \quad, \quad \eta=\frac{\eta_{0}}{1+\widetilde{\phi}^{2}}
$$

where $\eta_{0}=\eta(\widetilde{\phi}=0)=m^{2} M_{P}^{2} / V_{0}$. The spectral index $\delta_{n s}$ and the tensor-to-scalar ratio $r$ are

$$
\delta_{n s}=4 \eta_{0} \frac{\widetilde{\phi}^{2}-1 / 2}{\left(1+\widetilde{\phi}^{2}\right)^{2}} \quad, \quad r=\frac{16 \eta_{0} \widetilde{\phi}^{2}}{\left(1+\widetilde{\phi}^{2}\right)^{2}} .
$$

It follows that

$$
r=\frac{4 \delta_{n s} \widetilde{\phi}^{2}}{\widetilde{\phi}^{2}-1 / 2}
$$

or

$$
\widetilde{\phi}^{2}=\frac{r / 2}{r-4 \delta_{n s}} .
$$

This requires $r>4 \delta_{n s}=0.128$ which is just the opposite to the corresponding Hilltop requirement. Hence this model seems to be ruled out by the BICEP2/Planck result.

It follows from Equations (4.48) and (6.15.5) that the running of the tensor-to-scalar ratio is

$$
\alpha_{r}=\frac{\widetilde{\phi}^{2}-1}{2 \widetilde{\phi}^{2}-1} \delta_{n s}
$$

which gives

$$
\widetilde{\phi}^{2}=\frac{\alpha_{r}-\delta_{n s}}{2 \alpha_{r}-\delta_{n s}}
$$

which requires $\alpha_{r}>\delta_{n s}$ or $\alpha_{r}<\delta_{n s} / 2$. This, too, is opposite to the corresponding condition of the Hilltop model. Both models have difficulties in satisfying all the observational constraints simultaneously.

\subsection{Brane Inflation}

Several authors have considered 5-dimensional universe models where the inflationary era is due to a collision between branes [144-148]. This induces an effective modified gravity theory in a 4-dimensional world. We shall here follow the slow roll description as presented by Maartens and Koyoma [149].

It is usual to apply the Hubble slow roll parameters in the theory of brane inflation. However, there are some inaccuracies in the literature concerning the slow roll parameters of the brane inflation. Therefore I will here deduce the brane version of the connection between the potential slow roll parameters as defined in Equation (3.1) and the Hubble slow roll parameters as defined in Equation (3.7).

The brane version of the Friedmann Equation (2.1) takes the form

$$
H^{2}=\frac{\kappa}{3}\left[\frac{1}{2} \dot{\phi}^{2}+V\left(1+\frac{V}{2 \lambda}\right)\right]
$$


where $\lambda$ is the tension of each brane. Differentiating this equation and using Equation (2.2) which is unchanged in brane cosmology, give the brane generalization of Equation (2.12)

$$
\dot{H}=-\frac{\kappa \dot{\phi}^{2}}{2}\left(1-\frac{V V^{\prime}}{3 \lambda H \dot{\phi}}\right) .
$$

Using that $\dot{H}=V^{\prime} \dot{\phi}$ we obtain the brane version of Equation (2.5)

$$
H \dot{\phi}=\frac{V V^{\prime}}{3 \lambda}-\frac{2}{\kappa} H H^{\prime}
$$

Inserting this into the previous equation gives

$$
\dot{H}=-\frac{(\kappa / 2) \dot{\phi}^{2}}{1-\frac{\kappa}{6 \lambda} \frac{V V^{\prime}}{H H^{\prime}}} .
$$

Inserting Equation (6.16.2) into the first Equation (3.12) we find the brane version of the first Hubble slow roll parameter

$$
\varepsilon_{H B}=\frac{2}{\kappa}\left(\frac{H^{\prime}}{H}\right)^{2}-\frac{V V^{\prime} H^{\prime}}{3 \lambda H^{3}}
$$

which generalizes Equation (3.7).

Using the slow roll approximation to express $\varepsilon_{H B}$ in terms of $V$ and $V^{\prime}$ we may neglect $\dot{\phi}^{2}$ in Equation (6.16.1) which then reduces to

$$
H^{2}=\frac{\kappa}{3} V\left(1+\frac{V}{2 \lambda}\right)
$$

This gives

$$
H H^{\prime}=\frac{\kappa}{6}\left(1+\frac{V}{\lambda}\right) V^{\prime}
$$

Inserting this into Equation (6.16.5) leads to

$$
\varepsilon_{H B}=\left[\frac{1+V / \lambda}{(1+V / 2 \lambda)^{2}}\right] \varepsilon,
$$

where $\varepsilon$ is defined in Equation (3.1). This is in agreement with the result of Maartens and Koyoma [149].

At low energies, $V<<\lambda$, the brane Hubble slow roll parameter reduce to the standard form, $\varepsilon_{H B} \approx \varepsilon$, but at high energies, $V>>\lambda$, it takes the form

$$
\varepsilon_{H B} \approx(4 \lambda / V) \varepsilon
$$

Hence in this limit $\varepsilon_{H B}<<\varepsilon$.

Maartens and Koyoma have taken Equation (3.13) as the definition of $\eta_{H}$. Using Equation (2.2) we then have

$$
\eta_{H}=3+\frac{V^{\prime}}{H \dot{\phi}} .
$$

Inserting the brane expression (6.16.3) for $H \dot{\phi}$ we get

$$
\eta_{H B}=\frac{H H^{\prime}-\frac{\kappa}{6}\left(1+\frac{V}{\lambda}\right) V^{\prime}}{H H^{\prime}-\frac{\kappa}{6 \lambda} V V^{\prime}} .
$$


Note that the slow roll Equation (2.15) gives $\eta_{H B}=0$ corresponding to $\ddot{\phi}=0$. In order to express $\eta_{H B}$ in terms of $\eta$ and $\varepsilon$ we need a more accurate calculation.

Solving Equation (2.2) with respect to $\dot{\phi}$, differentiating with respect to time and using the expressions for $\eta_{H B}$ and $\varepsilon_{H B}$, we obtain

$$
-H \dot{\phi} \eta_{H B}=-\frac{V^{\prime}}{3} \varepsilon_{H B}-\frac{V^{\prime \prime} \dot{\phi}}{3 H}-\frac{\dddot{\phi}}{3 H}+\frac{1}{3} H \dot{\phi} \varepsilon_{H B} \eta_{H B} .
$$

We now use the slow roll approximation in the following way. It is assumed that $\dddot{\phi}$ is small to the second order in the slow roll parameters and can be neglected. Also the last term is small to the second order. Furthermore assuming that $\dddot{\phi}$ is small to the first order in the slow roll parameters, we make only a second order error by neglecting $\dddot{\phi}$ in Equation (2.2) so that we can use the approximation $H \dot{\phi}=-V^{\prime} / 3$ on the left hand and in second term on the right hand side. This gives

$$
\eta_{H B}=\frac{V^{\prime \prime}}{3 H^{2}}-\varepsilon_{H B}
$$

Again we make only a second order error by using Equation (6.16.6) in this equation, giving

$$
\eta_{H B}=\frac{V^{\prime \prime}}{\kappa V\left(1+\frac{V}{2 \lambda}\right)}-\varepsilon_{H B}
$$

Using the definition (3.1) of $\eta$ and Equation (6.16.8) this may be written,

$$
\eta_{H B}=\frac{\eta}{1+\frac{V}{2 \lambda}}-\frac{1+\frac{V}{\lambda}}{\left(1+\frac{V}{2 \lambda}\right)^{2}} \varepsilon
$$

which is different from the corresponding Equation (6.16.8) in Maartens and Koyoma [149]. At low energies, $V<<\lambda$, we have $\eta_{H B} \approx \eta-\varepsilon$ in agreement with Equation (3.22) since $\varepsilon \approx \varepsilon_{H}$. At high energies, $V>>\lambda$, Equation (6.16.15) gives

$$
\eta_{H B} \approx \frac{2 \lambda}{V}(\eta-2 \varepsilon)
$$

Hence, in this limit $\eta_{H B}$ has much smaller values than $\eta$ and $\varepsilon$. With the polynomial potential above we obtain

$$
\varepsilon_{H B} \approx \frac{8 \lambda}{\sigma \phi^{4}}, \varepsilon_{H B} \approx \frac{-4 \lambda}{\sigma \phi^{4}} .
$$

From Equations (4.20), (6.16.9) and (6.16.16) we have

$$
\delta_{n s} \approx \frac{4 \lambda}{V}(6 \varepsilon-\eta)
$$

The number of e-folds during inflation is

$$
N=\kappa \int_{\phi_{f}}^{\phi} \frac{V}{V^{\prime}}\left(1+\frac{V}{2 \lambda}\right) d \phi .
$$

For $V>>\lambda$ this reduces to

$$
N \approx \frac{\kappa}{2 \lambda} \int_{\phi_{f}}^{\phi} \frac{V^{2}}{V^{\prime}} d \phi
$$


Let us consider polynomial inflation with potential given in Equation (6.1.1). The low energy regime is similar to ordinary chaotic inflation as described in Section 6.1, so we shall here consider the high energy regime with $V>>\lambda$. Then Equation (6.16.20) gives

$$
N=\frac{\sigma M_{P}^{4}}{2 p(p+2) \lambda}\left[\left(\frac{\phi}{M_{P}}\right)^{p+2}-\left(\frac{\phi_{f}}{M_{P}}\right)^{p+2}\right] .
$$

Assuming that that the inflation ends at a much lower value of the field than the initial value, we can neglect the last term, which gives

$$
\left(\frac{\phi}{M_{P}}\right)^{p+2}=\frac{2 p(p+2) \lambda N}{\sigma M_{P}^{4}} .
$$

Differentiating the potential we get

$$
\varepsilon=\frac{p^{2} M_{P}^{2}}{2 \phi^{2}}, \eta=\frac{p(p-1) M_{P}^{2}}{\phi^{2}} .
$$

Inserting this into Equation (6.16.18) gives

$$
\delta_{n s B} \approx \frac{4 \lambda p(2 p+1)}{\sigma M_{P}^{4}}\left(\frac{M_{P}}{\phi}\right)^{p+2} .
$$

From Equations (6.15.19), (6.16.22) and (6.16.24) we get

$$
\varepsilon_{H B} \approx \frac{p}{p+2} \frac{1}{N} \quad, \quad \eta_{H B} \approx-\frac{1}{(p+2) N} \quad, \quad \delta_{n s B} \approx \frac{2(2 p+1)}{p+2} \frac{1}{N}
$$

in agreement with the results of Okada and Okada [148].

The Brane inflation models have a tensor-to-scalar ratio [150]

$$
r_{B}=24 \varepsilon_{H B},
$$

giving

$$
r_{B}=\frac{24 p}{p+2} \frac{1}{N}
$$

The relationship between the tensor-to-scalar ratio and the scalar frequency index is

$$
r_{B}=\frac{12 p}{2 p+1} \delta_{n s}
$$

or

$$
p=\frac{r_{B}}{2\left(6 \delta_{n s}-r_{B}\right)} .
$$

It follow from Equations (6.16.26) and (6.16.28) that

$$
r_{B}=8\left(\delta_{n s}-\frac{1}{N}\right)
$$

With $\delta_{n s}=0.032, N=50$ the chaotic brane inflation model predicts $r_{B}=0.096$. With these values of $\delta_{n s}$ and $r_{B}$ Equation (6.16.28) gives $p=0.5$.

It follows from Equations (4.29) and (6.15.25) that the running of the spectral indices are

$$
\alpha_{S B} \approx \frac{2(2 p+1) N}{p+2} \frac{1}{N_{2}} .
$$


For $0<p<\infty$ this gives $0.00066<\alpha_{S B}<0.0026$.

\subsection{Fast Roll Inflation}

H. Motohashi, A. A. Starobinsky and J. Yokoyama [107] have investigated a class of inflationary universe models given by a potential similar to that of hybrid inflation, but with the trigonometric functions replaced by hyperbolic functions,

$$
V(\phi)=(1 / 2) M^{2} M_{P}^{2}[6+\alpha-\alpha \cosh (\sqrt{2(3+\alpha)} \hat{\phi})]=M^{2} M_{P}^{2}\left[3-\alpha \sinh ^{2}\left(\sqrt{\frac{3+\alpha}{2}} \hat{\phi}\right)\right] .
$$

Essentially the same model was considered by Santos and Moraes [151]. Here $\alpha$ is a free parameter of this class of models that interpolate between $\alpha=0$ for a flat potential and $\alpha \simeq-3$ for the standard slow roll approximation. Furthermore they make the ansatz,

$$
\ddot{\phi}=-(3+\alpha) H \dot{\phi}
$$

Integration of this equation gives a relation between the scale factor and the time derivative of the inflaton field.

$$
a^{3+\alpha} \dot{\phi}=K_{1}
$$

where $K_{1}$ is an integration constant. Comparison of Equations (2.5) and (6.17.2) gives

$$
\alpha H \dot{\phi}=V^{\prime} .
$$

Substituting from Equation (2.13) for $\dot{\phi}$ gives

$$
2 \alpha M_{P}^{2} H H^{\prime}=-V^{\prime} .
$$

Integration leads to

$$
\alpha M_{P}^{2} H^{2}=-V+K_{2}
$$

Determining the integration constant from $H(0)=M$ gives $K_{2}=(3+\alpha) M_{P}^{2} M^{2}$. Then the Hubble parameter may be written as

$$
H(\phi)=M \cosh \left(\sqrt{\frac{3+\alpha}{2}} \hat{\phi}\right) .
$$

The constant $M$ represents the energy scale at the beginning of the inflationary era when the slow roll parameters are evaluated.

Differentiating $H$ and inserting the resulting expression for $H^{\prime}$ into Equation (2.13) gives

$$
\dot{\phi}=-\sqrt{2(3+\alpha)} M M_{P} \sinh \left(\sqrt{\frac{3+\alpha}{2}} \hat{\phi}\right) .
$$

Inserting this expression into Equation (6.17.3) and choosing $K_{1}=-M_{P} M \sqrt{2(3+\alpha)}$ gives the scale factor in terms of the inflaton field

$$
a(\phi)=\sinh ^{-1 /(3+\alpha)}\left(\sqrt{\frac{3+\alpha}{2}} \hat{\phi}\right) .
$$

Integration of Equation (6.17.8) gives

$$
\tanh \left(\frac{1}{2} \sqrt{\frac{3+\alpha}{2}} \frac{\phi}{M_{P}}\right)=K e^{-(3+\alpha) M t} .
$$


where $K$ is an integration constant. Hence, the inflaton field decreases with time and $\lim _{t \rightarrow \infty} \phi=0$. With the initial condition $\phi(0)=\infty$ we get $K=1$. Then the inflaton field as a function of time can then be written in several useful ways,

$$
\begin{aligned}
& \sinh (\sqrt{(3+\alpha) / 2} \hat{\phi})=1 / \sinh [(3+\alpha) M t], \\
& \cosh (\sqrt{(3+\alpha) / 2} \hat{\phi})=\operatorname{coth}[(3+\alpha) M t] \\
& \tanh (\sqrt{(3+\alpha) / 2} \hat{\phi})=1 / \cosh [(3+\alpha) M t]
\end{aligned}
$$

Inserting the first of these expressions into Equation (6.17.9) gives the scale factor as a function of time,

$$
a(t)=\sinh ^{1 /(3+\alpha)}[(3+\alpha) M t]
$$

and inserting the second of the expressions (6.17.11) into Equation (6.17.7) gives the Hubble parameter as a function of time

$$
H(t)=M \operatorname{coth}[(3+\alpha) M t]
$$

Note that for $\alpha=-3 / 2$ the scale factor of this class of inflationary models has the same time dependence,

$$
a(t)=\sinh ^{2 / 3}[(3 / 2) M t]
$$

as that of the standard $\Lambda$ CDM universe model dominated by dust and LIVE [152].

The horizon-flow slow roll parameters $\varepsilon_{n}$ can now be calculated from the definition (3.63) and the expression (6.17.13) for the Hubble parameter, giving

$$
\varepsilon_{1}=\varepsilon_{H}=\frac{3+\alpha}{\cosh ^{2}[(3+\alpha) M t]}=\frac{3+\alpha}{1+a^{2(3+\alpha)}} \quad, \quad \varepsilon_{2}=-2(3+\alpha) \tanh ^{2}[(3+\alpha) M t],
$$

correcting a minor printing error in [107] in the expression for $\varepsilon_{2}$. For $n \geq 1$ we get

$$
\varepsilon_{2 n}=2 \varepsilon_{1}-2(3+\alpha) \quad, \quad \varepsilon_{2 n+1}=2 \varepsilon_{1} .
$$

Hence $\lim _{t \rightarrow \infty} \varepsilon_{2 n+1}=2 \lim _{t \rightarrow \infty} \varepsilon_{1}=0, \lim _{t \rightarrow \infty} \varepsilon_{2 n}=-2(3+\alpha)$. Thus, as pointed out by Motohashi et al. [107], the even "slow roll" parameters are not small in these inflationary models.

The initial values of the slow roll parameters in the inflationary era are $\varepsilon_{1}(0)=3+\alpha, \varepsilon_{2}(0)=0$. Inserting this into Equation (4.57) gives

$$
\delta_{n s}=2(3+\alpha) \quad, \quad r=8 \delta_{n s}, \quad \alpha_{S}=-6 \delta_{n s}^{2}, \quad \alpha_{T}=2 \delta_{n s}^{2} .
$$

With the Planck 2015 value $\delta_{n s}=0.032$ we get $r=0.256, \alpha_{S}=-0.006, \alpha_{T}=0.002$. Also we then have $\alpha=-2.98$, and the quantity $3+\alpha$ appearing in many of the expressions above, has a small value, $3+\alpha=0.02$. Then the potential (6.17.1) is close to the constant potential case represented by a cosmological constant. In this case also the even slow roll parameters are rather small, so the rolling is not so fast after all. Unfortunately this nice model is ruled out by the BKP-data due to the high value it predicts for $r$.

We shall now show that by taking the number of e-folds into consideration we arrive at a very similar set of predictions. Inserting the expression (16.17.13) for the Hubble parameter into Equation (3.62), performing the integration gives and utilizing Equation (16.17.11), leads to

$$
N=\frac{1}{3+\alpha} \ln \frac{\sinh [\sqrt{(3+\alpha) / 2} \hat{\phi}]}{\sinh \left[\sqrt{(3+\alpha) / 2} \hat{\phi}_{f}\right]} .
$$


Equation (6.17.15) shows that $\varepsilon_{1}<3+\alpha$, so with the Planck data the final value of the inflaton field cannot be given by the usual condition $\varepsilon_{1}\left(\phi_{f}\right)=1$. In their diagrams Motohashi et al. (2014) have considered the model during the time interval from $t=0$ until $t=2 / M$. Hence we determine $\phi_{f}$ from the first expression in Equation (6.16.11) with $M t=2$, giving

$$
\sinh \left(\sqrt{\frac{3+\alpha}{2}} \hat{\phi}_{f}\right)=\frac{1}{\sinh [2(3+\alpha)]}
$$

Inserting this into Equation (6.17.18) we get

$$
\sinh \left(\sqrt{\frac{3+\alpha}{2}} \hat{\phi}_{f}\right)=\frac{e^{(3+\alpha) N}}{\sinh [2(3+\alpha)]}
$$

and further inserting this into Equation (6.17.15) finally gives

$$
\varepsilon_{1}=\frac{3+\alpha}{1+e^{-2(3+\alpha) N} \sinh ^{2}[2(3+\alpha)]} .
$$

For $3+\alpha=0.02$ we have $\sinh ^{2}[2(3+\alpha)]=0.0016$, and hence for $N=50$ we then get $e^{-2(3+\alpha) N} \sinh ^{2}[2(3+\alpha)]=2.2 \cdot 10^{-4}$ leading to nearly the same predictions of the spectral parameters as in Equation (6.17.17).

It seems natural now to construct a hybrid natural inflationary universe model with potential (6.5.1) instead of (6.17.1). Then the hyperbolic function in Equation (6.17.7) would be replaced by a trigonometric function. However, it may be shown that all models of this type have a negative Hubble parameter. Hence, they are contracting universe models.

\subsection{Running Mass Inflation}

This is a supersymmetry motivated class of inflationary models. The simplest version has the potential $[153,154]$

$$
V(\phi)=V_{0}\left[1-\frac{\phi^{2}}{M^{2}}\left(\ln \frac{\phi}{\phi_{0}}-\frac{1}{2}\right)\right] .
$$

Here $V_{0}, M$ and $\phi_{0}$ are three free parameters, $M$ represents an energy scale and $\phi=\phi_{0}$ is an extremum of $V(\phi)$. Defining $\hat{\phi}=\phi / \phi_{0}, \quad b=\left(M_{P} / M\right)^{2}$, the slow roll parameters may be written as

$$
\varepsilon=2 b^{2}\left(\frac{\phi}{M_{P}}\right)^{2}(\ln \hat{\phi})^{2}, \quad \eta=-2 b(1+\ln \hat{\phi}) \quad, \quad \xi=4 b^{2} \ln \hat{\phi} .
$$

Note that $\eta<0$. The expressions (6.18.2) give

$$
\delta_{n s}=4 b\left[1+\ln \hat{\phi}+3 b\left(\frac{\phi}{M_{P}}\right)^{2}(\ln \hat{\phi})^{2}\right], \quad r=32 b^{2}\left(\frac{\phi}{M_{P}}\right)^{2}(\ln \hat{\phi})^{2} .
$$

The observed values of $\delta_{n s}$ and $r$ can be used to restrict the parameter $M$ and the field strenth $\phi$ of this class of models. Inverting the expressions for $\delta_{n s}$ and $r$ we get

$$
\ln \hat{\phi}=\frac{\delta_{n s}-\frac{3}{8} r-4 b}{4 b},\left(\frac{\phi}{M_{P}}\right)^{2}=\frac{r}{2\left[\delta_{n s}-\frac{3}{8} r-4 b\right]^{2}} .
$$

Hence it is necessary that

$$
b<\frac{1}{4}\left[\delta_{n s}-\frac{3}{8} r\right]<\frac{1}{4} \delta_{n s}
$$


Inserting the Planck/BICEP2 results gives $b<0.008$ or $M>11 M_{P}$ and $\phi<11 M_{P}$.

\subsection{9. $k$-Inflation}

The usual models of the inflationary era describe it as a "slow roll" era where the potential energy of the inflaton field dominates over its kinetic energy and drives an accelerated and nearly exponential expansion of the universe.

In 1999 V. F. Mukhanov and coworkers [155,156] introduced a string theory inspired class of inflation models where the kinetic energy of the inflaton field, i.e., the square of the time derivative of the scalar field, drives the accelerated expansion. It was called k-inflation.

In this theory there appears a new parameter, $c_{s}$, the velocity of the sound waves in the perturbed inflaton field. One then defines a quantity representing the ratio of the velocity of the sound waves and the ratio of the cosmic expansion,

$$
s \equiv \frac{\left(\ln c_{s}\right)^{\cdot}}{(\ln a)^{\cdot}}=\frac{\dot{c}_{s}}{H c_{s}}
$$

The expression for the scalar tensor index, as given in Equation (4.56), is generalized to [157],

$$
\delta_{n s}=2 \varepsilon_{1}+\varepsilon_{2}+s .
$$

Lorenz et al. [158] have shown that to lowest order the tensor to scalar ratio is

$$
r=16 c_{s} \varepsilon_{1}
$$

From the relationship (4.15) with $\varepsilon_{1} \approx \varepsilon$ we then have

$$
n_{T}=r / 8 c_{s} .
$$

Tsjujikawa has discussed a model of this type with

$$
\delta_{n s}=\frac{24 c_{s}^{2}}{1+3 c_{s}^{2}} \quad, \quad r=\frac{192 c_{s}^{2}}{1+3 c_{s}^{2}} .
$$

For this model the $\delta_{n s, r}-$ relationship is $r=8 \delta_{n s}$, and the Planck 2015 value $\delta_{n s}=0.032$ gives $r=0.256$ which is too large according to the Planck/BICEP2 data.

\subsection{Dirac-Born-Infield (DBI) Inflation}

This is a string theory inspired class of inflationary models. We shall here only summarize the results of Li and Liddle [157] concerning the spectral parameters of such models. They considered a class of DBI-inflationary models with polynomial potential $V \propto \phi^{p}$ and deduced the following expressions for the scalar spectral index and the tensor-to-scalar ratio,

$$
\delta_{n s}=\left(1+\frac{p}{\beta}\right) \frac{1}{N} \quad, \quad r=\frac{8 p}{\beta N} \quad, \quad \beta=\frac{1}{2}\left(1-c_{s}^{2}\right) p+1+c_{s}^{2} .
$$

Here $c_{S}$ is the sound velocity in the cosmic plasma. It follows from these expressions that

$$
r=8\left(\delta_{n s}-\frac{1}{N}\right) .
$$

The running of the tensor-to-scalar ratio is

$$
T_{r}=1 / N
$$


With $\delta_{n s}=0.032, N=50$ we get $r=0.096$ and $T_{r}=0.02$. These predictions are independent of the value of $p$. Solving the last expression in Equation (5.21.1) with respect to $c_{s}^{2}$ we get

$$
c_{s}^{2}=\frac{2 \beta-2-p}{2-p} .
$$

Hence values of $p$ between 2 and $2 \beta-2$ are not allowed. Further observational restrictions of the DBI-models have been discussed by Tsujikawa [159].

\subsection{Fluxbrane Inflation}

Taking into account radiative corrections Martin et al. [33] has argued that one can consider an inflationary model where the inflaton field has the potential

$$
V(\phi)=V_{0}(1+\alpha \ln \hat{\phi}) .
$$

where $\hat{\phi}=\kappa \phi$. This has also been considered by Lyth and Riotto [22] and is called 'spontaneously broken SUSY inflation. This model has also been considered by Guo and Zhang [160]. An inflation model with this form of the potential has also been considered by Hebecker et al. [161].

In the potential (6.21.1) $\alpha$ is a dimensional parameter which represents the strength of the radiative effects. It is usually assumed that $\alpha>0$ and $\alpha<<1$. Hence we can use the approximations

$$
\varepsilon \equiv \frac{1}{2 \kappa}\left(\frac{V^{\prime}}{V_{0}}\right)^{2}, \quad \eta \equiv \frac{1}{\kappa} \frac{V^{\prime \prime}}{V_{0}}
$$

for the slow roll parameters. We then get

$$
\varepsilon \approx \frac{\alpha^{2}}{2 \hat{\phi}^{2}} \quad, \quad \eta \approx-\frac{\alpha}{\hat{\phi}^{2}} \quad, \quad \xi=\frac{2 \alpha^{2}}{\hat{\phi}^{4}} .
$$

In this case $\varepsilon<<|\eta|$, and we can approximate $\delta_{n s}$ by $\delta_{n s} \approx-2 \eta$. This gives

$$
\delta_{n s} \approx \frac{2 \alpha}{\hat{\phi}^{2}}, \quad r \approx \frac{8 \alpha^{2}}{\hat{\phi}^{2}}, \quad \alpha_{S}=-\frac{6 \alpha^{3}(2+\alpha)}{\hat{\phi}^{4}} .
$$

Hence the $\delta_{n s}, r-$ and $\delta_{n s}, \alpha_{S}-$ relations are

$$
r=4 \alpha \delta_{n s} \quad, \quad \alpha_{S}=-(3 / 2) \alpha(2+\alpha) \delta_{n s}^{2} .
$$

It follows from these expressions that

$$
\alpha_{S}=-\frac{3 r}{32}\left(8 \delta_{n s}+r\right) \quad, \quad \alpha_{r}=\left(1-\frac{\alpha}{2}\right) \delta_{n s} .
$$

The Planck value $\delta_{n s}=0.032$ and $\alpha<<1$ we get $r<<\delta_{n s}$ and $\alpha_{r} \approx 0.032$.

With the potential (6.21.1) the number of e-folds is approximately

$$
N \approx(1 / 2 \alpha)\left(\hat{\phi}^{2}-\hat{\phi}_{f}^{2}\right)
$$

The value of the inflaton field at the end of the slow roll era is given by $\varepsilon\left(\hat{\phi}_{f}\right)=1$, which leads to

$$
\phi_{f}^{2}=\alpha^{2} / 2
$$


Hence the value of the inflaton field during the slow roll era is given by

$$
\hat{\phi}^{2}=\alpha^{2} / 2+2 \alpha N \approx 2 \alpha N .
$$

Inserting this into Equation (6.21.4) gives

$$
\delta_{n s} \approx \frac{1}{N} \quad, \quad r \approx \frac{4 \alpha}{N} .
$$

With $\delta_{n s}=0.032$ we get $N=31$ which is lower than admitted in order to solve the horizon- and flatness problems.

\subsection{Mutated Hilltop Inflation}

B. K. Pal et al. $[162,163]$ have introduced a new supergravity inspired model of inflation which they have called mutated hilltop inflation. In this model the inflaton field has potential

$$
V(\phi)=V_{0}[1-1 / \cosh (\alpha \hat{\phi})] .
$$

Here $V_{0}$ represents the typical energy scale for hilltop inflation, $V_{0}^{1 / 4} \sim 10^{16} \mathrm{GeV}$, and $\alpha$ is a dimensionless parameter which characterizes the energy at the beginning of the slow roll era. Using the slow roll approximation in the form of Equation (2.61) the square of the Hubble parameter is

$$
H^{2}(\phi)=\frac{V_{0}}{3 M_{P}^{2}}[1-1 / \cosh (\alpha \hat{\phi})]
$$

Differentiating and inserting the resulting expressions into Equation (3.7), the Hubble slow roll parameters for this model come out as

$$
\begin{aligned}
& \varepsilon_{H}=\frac{M_{P}^{2} \alpha^{2}}{2} \frac{\tanh ^{2}(\alpha \hat{\phi})}{[\cosh (\alpha \hat{\phi})-1]^{2}}, \\
& \eta_{H}=M_{P}^{2} \alpha^{2} \frac{2-\cosh ^{2}(\alpha \hat{\phi})}{\cosh ^{2}(\alpha \hat{\phi})[\cosh (\alpha \hat{\phi})-1]}-\varepsilon_{H}=-\frac{M_{P}^{2} \alpha^{2}}{2} \frac{3+2 \cosh (\alpha \hat{\phi})}{\cosh ^{2}(\alpha \hat{\phi})} .
\end{aligned}
$$

Pal et al. considered in particular the large field case with $\cosh (\alpha \hat{\phi}) \approx e^{\alpha \hat{\phi}} / 2>>1$ and $\tanh (\alpha \hat{\phi}) \approx 1$. Then $\varepsilon_{H}<<\eta_{H}$ and the expressions for the slow roll parameters reduce to

$$
\varepsilon_{H} \approx 2 \alpha^{2} e^{-2 \alpha \hat{\phi}}, \quad \eta_{H} \approx-2 \alpha^{2} e^{-\alpha \hat{\phi}} .
$$

Hence according to Equation (4.16), in this approximation we have

$$
\delta_{n s} \approx-2 \eta_{H}=4 \alpha^{2} e^{-\alpha \hat{\phi}}, \quad r=16 \varepsilon_{H} \approx 32 \alpha^{2} e^{-2 \alpha \hat{\phi}} .
$$

The $\delta_{n s}, r$ - relationship then takes the form

$$
r \approx\left(2 / \alpha^{2}\right) \delta_{n s}^{2}
$$

Hence, the running of the tensor-to-scalar ratio is

$$
T_{r}=\left(1-\frac{\delta_{n s}}{4 \alpha^{2}}\right) \delta_{n s}
$$

B. K. Pal et al. [162] have considered models with $\alpha \approx 3$. With the Planck/BICEP2 value $\delta_{n s}=0.032$ we then get a very small value for the tensor-to-scalar ratio, $r=1.1 \times 10^{-4}$. 
We have an approximate expression for the number of e-folds

$$
N=\frac{1}{M_{P}} \int_{\hat{\phi}_{f}}^{\hat{\phi}} \frac{d \phi}{\sqrt{2 \varepsilon_{H}}}=\left(1 / 2 \alpha^{2}\right)\left(e^{\alpha \hat{\phi}}-e^{\alpha \hat{\phi}_{f}}\right) .
$$

The value of the inflaton field at the end of the slow roll era is given by $\varepsilon_{H}\left(\hat{\phi}_{f}\right)=1$, giving

$$
e^{\alpha \hat{\phi}_{f}}=\sqrt{2} \alpha
$$

Inserting this into Equation (6.22.7) gives the inflaton field during the slow roll era,

$$
e^{\alpha \hat{\phi}}=2 \alpha^{2} N+\sqrt{2} \alpha \approx 2 \alpha^{2} N
$$

From this equation in combination with Equation (6.22.5) we get

$$
\delta_{n s} \approx \frac{2}{N}, r \approx \frac{8}{\alpha^{2} N^{2}}
$$

With $\delta_{n s}=0.032$ we get $N=62.5$. This model is not ruled out by the Planck/BICEP2 observations.

\subsection{Arctan Inflation}

In a toy-model of inflation of the large field type, called arctan inflation by Drees and Erfani [101] and by Martin et al. [33], the inflaton potential is

$$
V(\phi)=V_{0}\left(1+\frac{2}{\pi} \arctan \bar{\phi}\right), \bar{\phi}=\phi / M,
$$

where the mass parameter $M$ is assumed to be much larger than the Planck mass, $M>>M_{P}$. It is also assumed that $\bar{\phi}>>1$, so that we can let $V(\bar{\phi})$ be approximated by $2 V_{0}$ in the in the expressions of the slow roll parameters. Defining $b=\left(M_{P} / M\right)^{2}$ we then get

$$
\varepsilon \simeq \frac{b}{2 \pi^{2}} \frac{1}{\bar{\phi}^{4}} \quad, \quad \eta \simeq-\frac{2 b}{\pi} \frac{1}{\bar{\phi}^{3}} \quad, \quad \xi \simeq \frac{6 b^{2}}{\pi^{2}} \frac{1}{\bar{\phi}^{6}} .
$$

It follows that $\varepsilon<<|\eta|$ and hence that $\varepsilon$ can be neglected in the expression for $\delta_{n s}$. This gives

$$
\delta_{n s} \simeq \frac{4 b}{\pi} \frac{1}{\bar{\phi}^{3}} \quad, \quad r \simeq \frac{8 b}{\pi^{2}} \frac{1}{\bar{\phi}^{4}} \quad, \quad \alpha_{S} \simeq-\frac{12 b^{2}}{\pi^{2}} \frac{1}{\bar{\phi}^{6}} .
$$

These expressions give the relationships

$$
r \simeq\left(\frac{2 b}{\pi^{2}}\right)^{1 / 3} \delta_{n s}^{4 / 3} \quad, \quad \alpha_{S}=-\frac{3}{4} \delta_{n s}^{2}
$$

Hence

$$
b \simeq \frac{\pi^{2}}{2} \frac{r^{3}}{\delta_{n s}^{4}}
$$

With the center values $\delta_{n s}=0.032$ and $r=0.05$ of Planck 2015 and Planck/BICEP we get $M \simeq 24 M_{P}$ and $\alpha_{S} \simeq-0.0008$. The value of $\alpha_{S}$ is in agreement with the data so far, but Martin et al (2013) have estimated the energy scale to be $M \simeq 10^{-3} M_{P}$ which requires $r=5.9 \times 10^{-5}$. 
The number of e-folds is

$$
N \simeq \frac{1}{M_{P}^{2}} \int_{\bar{\phi}_{f}}^{\bar{\phi}} \frac{2 V_{0}}{V^{\prime}} d \phi \simeq \frac{\pi}{3 b}\left(\bar{\phi}^{3}-\bar{\phi}_{f}^{3}\right)
$$

Assuming that $\bar{\phi}_{f}^{3}<<\bar{\phi}^{3}$ the value of the inflaton field during the slow roll era is given by

$$
\bar{\phi}^{3} \simeq \frac{3 b}{\pi} N
$$

Inserting this into Equation (6.23.3) the spectral parameters are found to be

$$
\delta_{n s} \simeq \frac{4}{3 N} \quad, \quad r=\left(\frac{1}{9 \pi}\right)^{2 / 3} b^{-1 / 3} \frac{8}{N^{4 / 3}} \quad, \quad \alpha_{S}=-\frac{4}{3} \frac{1}{N^{2}}
$$

With $\delta_{n s}=0.032$ the first of these relationships implies that the number of e-folds during the slow roll era is $N=42$ which is a little less that the optimal number for solving the horizon and flatness problems.

\subsection{Inflation with Fractional Potential}

Eshagli et al. [164] have investigated an inflation model in which the inflaton field has a fractional potential,

$$
V(\phi)=V_{0} \frac{\alpha \hat{\phi}^{2}}{1+\alpha \hat{\phi}^{2}}
$$

where $\alpha$ is an arbitrary dimensionless constant. It is assumed that $\alpha \hat{\phi}^{2}>>1$ during the slow roll era, so that the slow roll parameters can be calculated with $V$ replaced by $V_{0}$ in the numerator in the defintions (3.1). Hence

$$
\varepsilon \simeq \frac{2}{\alpha^{2}} \frac{1}{\hat{\phi}^{6}} \quad, \quad \eta \simeq-\frac{6}{\alpha} \frac{1}{\hat{\phi}^{4}} \quad, \quad \xi \simeq \frac{48}{\alpha^{2}} \frac{1}{\hat{\phi}^{8}} .
$$

The slow roll era ends when the inflaton field has a value $\hat{\phi}_{f}=\left(2 / \alpha^{2}\right)^{1 / 6}$. The number of e-folds during the slow roll era is

$$
N=\frac{\alpha}{8}\left(\hat{\phi}^{4}-\hat{\phi}_{f}^{4}\right)=\frac{\alpha}{8}\left[\hat{\phi}^{4}-\left(2 / \alpha^{2}\right)^{2 / 3}\right]
$$

Assuming that the last term can be neglected compared to the first the value of the inflaton field during the slow roll parameter is $\hat{\phi} \simeq(8 N / \alpha)^{1 / 4}$. Inserting this into Equation (6.24.2) gives

$$
\varepsilon \simeq \frac{1}{8 \sqrt{2 \alpha}} \frac{1}{N^{3 / 2}} \quad, \quad \eta \simeq-\frac{3}{4 N} \quad, \quad \xi \simeq \frac{3}{4} \frac{1}{N^{2}} .
$$

The spectral parameters are

$$
\delta_{n s} \simeq \frac{3}{2 N}\left(1+\frac{1}{2 \sqrt{2 \alpha N}}\right) \quad, \quad r \simeq \sqrt{\frac{2}{\alpha}} \frac{1}{N^{3 / 2}} \quad, \quad \alpha_{S} \simeq-\frac{3}{2} \frac{1}{N^{2}}\left(1+\frac{1}{\sqrt{2 \alpha N}}+\frac{1}{8 \alpha N}\right) .
$$

Neglecting the last term in the expression for $\alpha_{S}$ this gives the consistency conditions

$$
r \simeq \frac{8}{3}\left(\delta_{n s}-\frac{3}{2 N}\right) \quad, \quad \alpha_{S} \simeq-\frac{1}{N}\left(\delta_{n s}+\frac{3}{8} r\right) \simeq-\frac{1}{N}\left(2 \delta_{n s}-\frac{3}{2 N}\right) .
$$


The running of the tensor-to-scalar ratio is

$$
T_{r}=\frac{2}{3} \delta_{n s}-\frac{1}{2 N}
$$

Inserting $N=50$ and $\delta_{n s}=0.032$ gives $r \simeq 0.005, \alpha_{S} \approx-0.0007$ and $T_{r}=0.01$ which is permitted by the BPK-data. The values of $r$ and $\alpha_{S}$ as functions of $N$ are shown in Figure 9 for $\delta_{n s}=0.032$. Note that $r>0$ requires $N>3 / 2 \delta_{n s} \approx 47$.
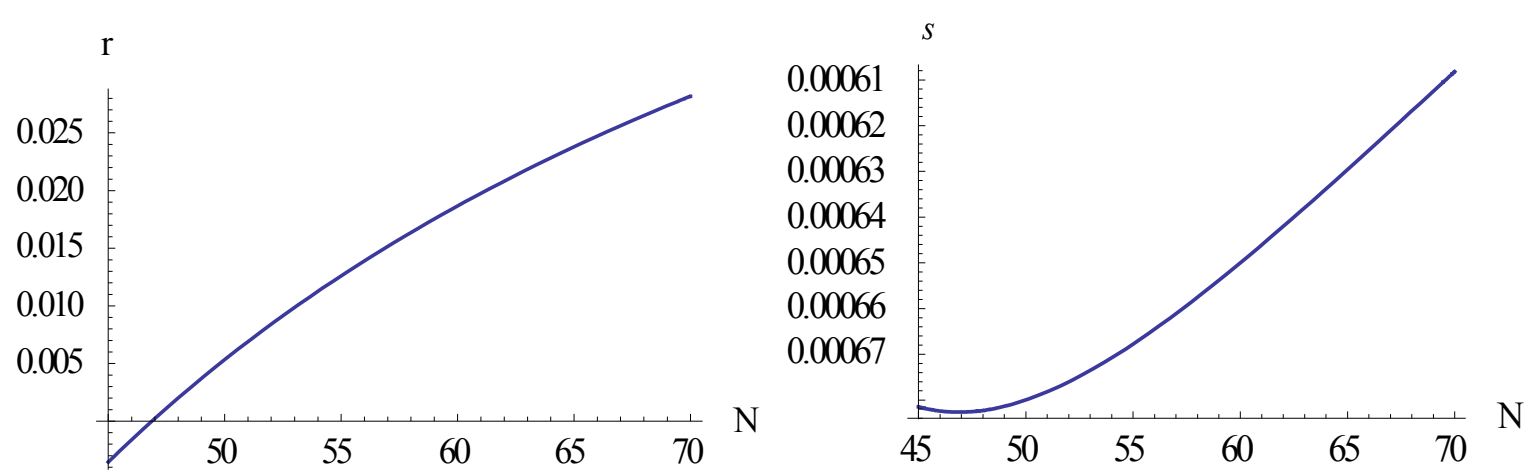

Figure 9. The spectral parameters $r$ and $\alpha_{S}$ as given in Equation (6.24.6).

We see that for $N<60$ the tensor-to-scalar ratio is $r<0.02$.

Two similar models called minimal Higgs inflation, have been investigated by Maity [165]. The first one has the potential

$$
V=\frac{\lambda}{4} \frac{\phi^{4}}{1+\hat{\phi}^{4}}
$$

where $\hat{\phi}=\phi / M$, and $M$ is the energy scale at which the universe enters the inflationary era. Defining again $b=\left(M_{P} / M\right)^{2}$ the potential slow roll parameters are

$$
\varepsilon=\frac{8 b}{\hat{\phi}^{2}\left(1+\hat{\phi}^{4}\right)^{2}} \quad, \quad \eta=12\left(\frac{M_{P}}{\phi}\right)^{2} \frac{1-\hat{\phi}^{4}}{\left(1+\hat{\phi}^{4}\right)^{2}} \quad, \quad \xi=96\left(\frac{M_{P}}{\phi}\right)^{4} \frac{1-8 \hat{\phi}^{4}+3 \hat{\phi}^{8}}{\left(1+\hat{\phi}^{4}\right)^{4}} .
$$

Assuming that $\hat{\phi}>>1$ the slow roll parameters can be approximated by

$$
\varepsilon \approx 8 b \frac{1}{\hat{\phi}^{10}}, \quad \eta \approx-12 b \frac{1}{\hat{\phi}^{6}} \quad, \quad \xi=288 b^{2} \frac{1}{\hat{\phi}^{12}} .
$$

It follows that $\xi<<\varepsilon<<|\eta|$. Using the approximation (6.24.10) and assuming that $\phi>>\phi_{\text {final }}$ the number of e-folds is found to be

$$
N \approx \frac{1}{24 b} \hat{\phi}^{6}
$$

Inserting this into Equation (6.24.10) gives

$$
\varepsilon \approx \frac{1}{4 \times 3^{5 / 3} b^{2 / 3}} \frac{1}{N^{5 / 3}}, \quad \eta \approx-\frac{1}{2 N} \quad, \quad \xi \approx \frac{9}{128 b} \frac{1}{N^{3}} .
$$

We then obtain

$$
\delta_{n s} \approx-2 \eta \approx \frac{1}{N} \quad, \quad r \approx \frac{4}{3^{5 / 3} b^{2 / 3}} \frac{1}{N^{5 / 3}} \quad, \quad \alpha_{s} \approx-\frac{2}{5^{5 / 3} b^{2 / 3}} \frac{1}{N^{8 / 3}} .
$$

For $M<<M_{P}$ this gives a small value of $r$, but the Planck value $\delta_{n s}=0.032$ gives $N \approx 31$ which is too small to give a realistic inflationary scenario. 
The other model considered by Maity [160] has the potential

$$
V=\frac{\lambda}{4} \frac{\phi^{4}}{\left(1+\hat{\phi}^{2}\right)^{2}} .
$$

For this model the slow roll parameters are

$$
\varepsilon=\frac{8 b}{\hat{\phi}^{2}\left(1+\hat{\phi}^{2}\right)^{2}} \quad, \quad \eta=12 b \frac{1-\hat{\phi}^{2}}{\hat{\phi}^{2}\left(1+\hat{\phi}^{2}\right)^{2}} \quad, \quad \xi=96 b^{2} \frac{1-6 \hat{\phi}^{2}+4 \hat{\phi}^{6}}{\hat{\phi}^{4}\left(1+\hat{\phi}^{2}\right)^{5}} .
$$

Again we have the large field approximation

$$
\varepsilon \approx 8 b \frac{1}{\hat{\phi}^{6}} \quad, \quad \eta \approx-12 b \frac{1}{\hat{\phi}^{4}} \quad, \quad \xi \approx 384 b^{2} \frac{1}{\hat{\phi}^{8}} .
$$

For this model the number of e-folds is

$$
N \approx \frac{1}{16 b} \hat{\phi}^{4} .
$$

Inserting this into Equation (6.24.16) gives

$$
\varepsilon \approx \frac{1}{8 b^{1 / 2}} \frac{1}{N^{3 / 2}} \quad, \quad \eta=-\frac{3}{4 N} \quad, \quad \xi \approx \frac{3}{2 N^{2}} .
$$

Hence, we get

$$
\delta_{n s} \approx \frac{3}{2 N} \quad, \quad r \approx \frac{2}{b^{1 / 2}} \frac{1}{N^{3 / 2}} \quad, \quad \alpha_{s}=-\frac{3}{N^{2}} .
$$

For this model the Planck value $\delta_{n s}=0.032$ gives $N \approx 47$ which may be acceptable. So this is a more promising model than the previous one. Also, for $M<<M_{P}$ this model predicts a small value of $r$.

\subsection{Twisted Inflation}

J. L. Davis et al. [80] have introduced an inflationary model motivated by brane cosmology which they have called twisted inflation. The argued that the potential of the inflaton field has the form

$$
V(\phi)=M^{4}\left(1-A \widetilde{\phi}^{2} e^{-\widetilde{\phi}}\right), \quad \widetilde{\phi}=\phi / \phi_{0}
$$

for $\phi>>1$. Calculating the spectral parameters there will appear second order polynoms in $\widetilde{\phi}$ times $e^{-\widetilde{\phi}}$ and times $e^{-2 \widetilde{\phi}}$. Then it is a sufficiently good approximation to keep only the terms with $\widetilde{\phi}^{2} e^{-\widetilde{\phi}}$. This gives

$$
\delta_{n s} \simeq 2\left(\frac{M_{P}}{\phi_{0}}\right)^{2} A \widetilde{\phi}^{2} e^{-\widetilde{\phi}} \quad, \quad r \simeq 8\left(\frac{M_{P}}{\phi_{0}}\right)^{2}\left(A \widetilde{\phi}^{2} e^{-\widetilde{\phi}}\right)^{2} \quad, \quad \alpha_{S} \simeq-2\left(\frac{M_{P}}{\phi_{0}}\right)^{4}\left(A \widetilde{\phi}^{2} e^{-\widetilde{\phi}}\right)^{2} .
$$

Hence

$$
r=2\left(\frac{\phi_{0}}{M_{P}}\right) \delta_{n s}^{2} \quad, \quad \alpha_{S}=-\frac{1}{2} \delta_{n s}^{2} .
$$

Martin et al. [33] have estimated that $\phi_{0} / M_{P} \simeq 10^{-5}$. This implies that the tensor-to-scalar ratio has a very small value according to the twisted inflation model. With $\delta_{n s}=0.032$ the running of the scalar spectral index is $\alpha_{S}=-0.0005$. According to Equation (4.50) the running of the tensor-to-scalar ratio is then approximately equal to $\delta_{n s}$, i.e., $\alpha_{r}=0.03$. 


\subsection{Inflation with Invariant Density Spectrum}

We shall here take a closer view upon the inflationary universe model with an inflaton potential of the form (6.21) giving a scale invariant density spectrum to first order in the slow roll parameters. The potential is here written as [33]

$$
V(\phi)=V_{0}(1-\alpha \hat{\phi})^{-2},
$$

where $\alpha$ is a free parameter. The slow roll parameters are

$$
\varepsilon=\frac{2 \alpha^{2}}{(1-\alpha \hat{\phi})^{2}} \quad, \quad \eta=\frac{6 \alpha^{2}}{(1-\alpha \hat{\phi})^{2}} \quad, \quad \xi=\frac{48 \alpha^{4}}{(1-\alpha \hat{\phi})^{4}}
$$

This shows that

$$
\eta=3 \varepsilon \quad, \quad \xi=12 \varepsilon^{2}
$$

Hence

$$
\delta_{n s}=\alpha_{S}=0 \quad, \quad r=\frac{32 \alpha^{2}}{(1-\alpha \hat{\phi})^{2}} .
$$

This shows that the inflationary model with inflaton potential (6.26.1) has a scale invariant Harrison-Zeldovich density fluctuation spectrum.

The slow roll era ends when the inflaton field obtains a value $\hat{\phi}_{f}$ given by $\varepsilon\left(\hat{\phi}_{f}\right)=1$, leading to

$$
\hat{\phi}_{f}=\frac{1 \pm \alpha \sqrt{2}}{\alpha} .
$$

The number of e-folds during the slow roll era is

$$
N(\phi)=\frac{1}{2 \alpha M_{P}} \int_{\hat{\phi}_{f}}^{\hat{\phi}}(1-\alpha \hat{\phi}) d \phi=(1 / 4 \alpha)\left(\hat{\phi}-\hat{\phi}_{f}\right)\left[2-\alpha\left(\hat{\phi}+\hat{\phi}_{f}\right)\right] .
$$

We assume that $\alpha>0$. Then, in order that $N>0$, we must have either $\hat{\phi}>\hat{\phi}_{f} \& \alpha \hat{\phi}<1$ with $\alpha \hat{\phi}_{f}=1-\alpha \sqrt{2}$ or $\hat{\phi}<\hat{\phi}_{f} \& \alpha \hat{\phi}>1$ with $\alpha \hat{\phi}_{f}=1+\alpha \sqrt{2}$. The maximal value of the function $N(\hat{\phi})$ with the values (6.26.5) for $\hat{\phi}_{f}$ is $N_{\max }=1 / 2$. Hence this model is ruled out as a realistic inflationary model.

\subsection{Quintessential Inflation}

Quintessential inflation has been considered by Md. W. Hossein et al. [166]. We shall here analyze this type of inflation by means of the $\mathrm{N}$-formalism. Hossein et al. found that in the small field approximation the first potential slow roll parameter is given in terms of the number of e-folds as

$$
\varepsilon(N)=\frac{\alpha^{2}}{2} \frac{1}{1-e^{-\alpha^{2} N}}
$$

where $\alpha$ is a parameter characterizing the energy of the inflaton field during the slow roll era. In the case of small field inflation $1 / N<<\alpha<<1$. From Equations (5.1), (5.3) and (6.27.1) we obtain

$$
\delta_{n s}=\alpha^{2} \operatorname{coth}\left(\alpha^{2} N / 2\right) \quad, \quad r=-8 n_{T}=\frac{8 \alpha^{2}}{1-e^{-\alpha^{2} N}} \quad, \quad \alpha_{S}=2 \alpha_{T}=-\frac{\alpha^{4}}{2} \frac{1}{\sinh ^{2}\left(\alpha^{2} N / 2\right)} .
$$


Inserting Equation (6.27.1) into Equations (5.4) and (5.7) and performing the integrations give

$$
V(N)=e^{\alpha^{2} N}-1
$$

and

$$
\phi(N)=\frac{2 M_{P}}{\alpha} \ln \left(e^{\alpha^{2} N / 2}+\sqrt{e^{\alpha^{2} N}-1}\right) .
$$

Inverting this equation gives

$$
e^{\alpha^{2} N / 2}=\cosh \left(\frac{\alpha}{2} \hat{\phi}\right) .
$$

Inserting this into Equation (6.27.3) shows that the potential of this inflationary model is

$$
V(\phi)=\sinh ^{2}\left(\frac{\alpha}{2} \hat{\phi}\right) .
$$

Hence this inflationary model is mathematically similar to hyperbolic inflation with $p=2$.

The expressions (6.27.2) give the consistency relation

$$
r=4\left(\delta_{n s}+\alpha^{2}\right)
$$

This shows that $r>4 \delta_{n s}$. Hence with $\delta_{n s}=0.032$ this model of quintessential inflation predicts $r>0.128$ which is ruled out by the BPK-data.

We shall also review three more recent versions of quintessential inflation, and consider first a model investigated by Bruck et al. [167]. The potential is

$$
V=\frac{V_{0}}{2}[1+\tanh (p \hat{\phi})] \quad, \quad p>0 .
$$

The potential slow roll parameters are

$$
\varepsilon=\frac{p^{2}}{2}[1-\tan (p \hat{\phi})]^{2}, \quad \eta=-2 p^{2} \tanh (p \hat{\phi})[1-\tan (p \hat{\phi})] .
$$

Hence the spectral parameters are,

$$
r=16 \varepsilon=8 p^{2}[1-\tanh (p \hat{\phi})]^{2}, \quad \delta_{n s}=2(3 \varepsilon-\eta)=p^{2}\left[3-2 \tanh (p \hat{\phi})-\tanh ^{2}(p \hat{\phi})\right]
$$

Calculating the number of e-folds, Bruck et al. [162] have shown that

$$
e^{2 p \hat{\phi}} \approx 4 p^{2} N
$$

Hence,

$$
\tanh (p \hat{\phi}) \approx \frac{4 p^{2} N-1}{4 p^{2} N+1}
$$

Inserting this into Equation (6.27.10) gives

$$
r \approx \frac{32 p^{2}}{\left(4 p^{2} N+1\right)^{2}} \approx \frac{2}{p^{2} N^{2}} \quad, \quad \delta_{n s} \approx 2 p^{2} \frac{3+16 p^{2} N}{\left(4 p^{2} N+1\right)^{2}} \approx \frac{2}{N} .
$$

Bruck et al. (2017) have suggested that $p \simeq 100$, so we can with good accuracy use the last approxomations in Equation (4.27.13). Inserting $\delta_{n s}=0.032$ gives $N \approx 62$ and $r \simeq 0$.

Next we consider a quintessence inflation model investigated by Dimopoulos [34], which has a potential

$$
V=M^{4} \exp \left(-2 n e^{\hat{\phi} / \sqrt{2} N_{1}}\right) .
$$


where $N_{1}$ and $n$ are dimensionless, positive constants. Note that Dimopoulos et al. used a constant $\alpha$ related to $N_{1}$ by $N_{1}=\sqrt{3 \alpha} / 2$ or $\alpha=(4 / 3) N_{1}^{2}$. Here $M^{4}=e^{2 n} V_{0}$ represents the inflation energy scale, where $V_{0}=V(0)$.

The potential slow roll parameters are

$$
\varepsilon=\left(\frac{n}{N_{1}}\right)^{2} e^{\sqrt{2} \hat{\phi} / N_{1}} \quad, \quad \eta=\frac{n}{N_{1}^{2}} e^{\hat{\phi} / \sqrt{2} N_{1}}\left(2 n e^{\hat{\phi} / \sqrt{2} N_{1}}-1\right) .
$$

The condition for the end of inflation, $\varepsilon\left(\phi_{f}\right)=1$ gives

$$
e^{\hat{\phi} / \sqrt{2} N_{1}}=\frac{N_{1}}{n} .
$$

The number of e-folds of the inflationary era is

$$
N=\frac{1}{\sqrt{2} M_{P}} \int_{\phi}^{\phi_{f}} \frac{1}{\sqrt{\varepsilon}} d \phi=\frac{N_{1}^{2}}{n}\left(e^{-\hat{\phi} / \sqrt{2} N_{1}}-e^{-\hat{\phi}_{f} / \sqrt{2} N_{1}}\right) .
$$

Inserting the value (4.27.16) for $\hat{\phi}_{f}$ gives

$$
e^{\hat{\phi} / \sqrt{2} N_{1}}=\frac{N_{1}^{2}}{n} \frac{1}{N+N_{1}} .
$$

Hence in terms of the number of e-folds the potential slow roll parameters are

$$
\varepsilon=\left(\frac{N_{1}}{N+N_{1}}\right)^{2}, \quad \eta=2 \varepsilon-\frac{1}{N+N_{1}} .
$$

Thus the scalar spectral index and the tensor-to-scalar ratio are

$$
\delta_{n s}=2\left(\frac{N_{1}}{N+N_{1}}\right)^{2}+\frac{2}{N+N_{1}} \quad, \quad r=16\left(\frac{N_{1}}{N+N_{1}}\right)^{2} .
$$

Equations (6.27.20) imply that

$$
N+N_{1}=\frac{16}{8 \delta_{n s}-r}
$$

which may be written

$$
N+N_{1}=\frac{4 N}{4-\sqrt{r}} .
$$

The last two equations gives an equation for $\sqrt{r}$ with positive solution

$$
\sqrt{r}=(2 / N)\left[1+\sqrt{1+2 N\left(N \delta_{n s}-2\right)}\right] .
$$

which requires

$$
N \delta_{n s}>1+\sqrt{1-(1 / 2) \delta_{n s}} \approx 2 .
$$

Inserting $\delta_{n s}=0.032$ gives $N>62.5$. With $N \delta_{n s}=2$ we get $r=4 \delta_{n s}^{2}=0.004$ and

$$
N_{1}=\frac{N \sqrt{r}}{4-\sqrt{r}}=\frac{N \delta_{n s}}{2-\delta_{n s}} \approx \frac{1}{2} N \delta_{n s}=1 .
$$

Hence in this case $\alpha=4 / 3$. 
Finally we consider a quintessence inflation model investigated by Agarwal et al. [134], which has a potential

$$
V(\phi)=\frac{V_{0}}{\cosh \left(\beta^{n} \hat{\phi}^{n}\right)} .
$$

For this model the potential slow roll parameters are

$$
\begin{aligned}
& \varepsilon=(1 / 2)\left[n \beta^{n} \hat{\phi}^{n-1} \tanh \left(\beta^{n} \hat{\phi}^{n}\right)\right]^{2}, \\
& \eta=-n \beta^{2}(\beta \hat{\phi})^{n-2}\left[n(\beta \hat{\phi})^{n}+(n-1) \tanh \left(\beta^{n} \hat{\phi}^{n}\right)-2 n(\beta \hat{\phi})^{n} \tanh ^{2}\left(\beta^{n} \hat{\phi}^{n}\right)\right] .
\end{aligned}
$$

Hence, the spectral parameters are

$$
\begin{aligned}
& \delta_{n s}=\beta^{n} \hat{\phi}^{n-2}\left[2 n^{2}(\beta \hat{\phi})^{n}+2 n(n-1) \tanh \left(\beta^{n} \hat{\phi}^{n}\right)-n^{2}(\beta \hat{\phi})^{n} \tanh ^{2}\left(\beta^{n} \hat{\phi}^{n}\right)\right], \\
& r=8\left[n \beta^{n} \hat{\phi}^{n-1} \tanh \left(\beta^{n} \hat{\phi}^{n}\right)\right]^{2} .
\end{aligned}
$$

The end of the inflationary era is defined by $\varepsilon\left(\hat{\phi}_{f}\right)=1$, which gives

$$
\left[n \beta^{n} \hat{\phi}_{f}^{n-1} \tanh \left(\beta^{n} \hat{\phi}_{f}^{n}\right)\right]^{2}=2 .
$$

In order to provide an analytical prediction Agarwal et al. [134] considered the case $n=1$. In this case Equation (6.27.29) reduces to

$$
\beta^{2} \tanh ^{2}(\beta \hat{\phi})=2 \text {. }
$$

Since $\tanh ^{2}(\beta \hat{\phi})<1$ this requires $\beta^{2}>2$ for inflation to end. In this case the spectral parameters are

$$
\delta_{n s}=\beta^{2}\left[2-\tanh ^{2}(\beta \hat{\phi})\right], \quad r=8 \beta^{2} \tanh ^{2}(\beta \hat{\phi}) .
$$

This leads to

$$
r=8\left(2 \beta^{2}-\delta_{n s}\right) .
$$

Hence $r>31.7$ which is totally unrealistic. Similar problems appear for higher values of $n$. Hence this class of inflationary models is ruled out by the requirement of a graceful end of the inflationary era.

\subsection{Generalized Chaplygin Gas (GCG) Inflation}

The GCG inflation model has recently been described by Dinda et al. [168]. We shall here give a review of their presentation.

The Generalized Chaplygin gas has a pressure $p$ which is given by the energy density $\rho$ as

$$
p=-A \rho^{1+\frac{m}{3}} .
$$

It follows from Equations (2.1) and (2.2) that the relationship of the density, pressure and scale factor for the Friedmann universe models can be written

$$
\frac{d \rho}{\rho+p}=-3 \frac{d a}{a} .
$$

Inserting Equation (6.28.1) and integrating gives

$$
\rho(a)=\left(A+B a^{m}\right)^{-3 / m} .
$$

For $m<0$ a universe dominated by Chaplygin gas behaves like a dust dominated universe model with $\rho \propto a^{-3}$ at very early times with small values of $a$, and as a universe dominated by LIVE with 
a constant density of the gas at late times with large values of $a$. For $m>0$ it is opposite. Hence the case $m<0$ may represent the period after the inflationary era, and the case $m>0$ can describe the evolution during the inflationary era. Hence we assume that $m>0$.

From Equations (2.1) and (6.28.1) we have

$$
\dot{\phi}^{2}=\rho+p=\rho\left(1-A \rho^{m / 3}\right) .
$$

Inserting Equation (6.28.3) gives.

$$
\dot{\phi}=\frac{\sqrt{\rho}}{\sqrt{1+(A / B) a^{-m}}} .
$$

The first part of Equation (2.2) may be written

$$
\dot{a}=a H=\frac{1}{M_{P} \sqrt{3}} a \sqrt{\rho} .
$$

From Equations (6.28.5) and (6.28.6) we have

$$
\frac{d \phi}{d a}=\frac{\dot{\phi}}{\dot{a}}=\frac{\sqrt{3} M_{P}}{a \sqrt{1+(A / B) a^{-m}}} .
$$

Integration gives

$$
A+B a^{m}=A \cosh ^{2} \breve{\phi}, \quad \breve{\phi}=\frac{m\left(\phi-\phi_{0}\right)}{2 \sqrt{3} M_{P}},
$$

where $\phi_{0}$ is an integration constant. Equation (6.28.8) implies that $a\left(\phi_{0}\right)=0$. Hence $\phi_{0}$ may be interpreted as the initial value of the inflaton field. From Equations (6.28.6) and (6.28.3) we have

$$
H=\frac{\sqrt{\rho}}{\sqrt{3} M_{P}}=\frac{1}{\sqrt{3} M_{P}} \frac{1}{\left(A+B a^{m}\right)^{3 / 2 m}} .
$$

Inserting Equation (6.28.8) gives

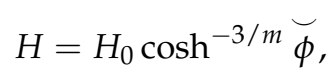

where $H_{0}=\left(1 / \sqrt{3} M_{P}\right) A^{-3 / 2 m}$ is the initial value of the Hubble parameter. Inserting Equation (4.28.10) into the first part of Equation (4.28.10) gives

$$
\rho(\phi)=A^{-3 / m} \cosh ^{-6 / m} \breve{\phi} .
$$

Hence, $A^{-3 / m}=\rho_{0}=\rho\left(\phi_{0}\right)$ in agreement with the equation $H_{0}=\sqrt{\rho_{0}} / \sqrt{3} M_{P}$. From Equations (2.1) and (6.29.1) we also have

$$
V=\frac{1}{2}(\rho-p)=\frac{\rho}{2}\left(1+A \rho^{m / 3}\right) .
$$

Inserting Equation (6.28.11) gives the inflaton potential

$$
V(\phi)=\left(V_{0} / 2\right) \frac{1+\cosh ^{2} \breve{\phi}}{\cosh ^{2(1+3 / m)} \stackrel{\phi}{~}},
$$

where $V_{0}=V\left(\phi_{0}\right)=A^{-3 / m}=\rho_{0}$. 
We now calculate the Hubble slow roll parameters. From Equations (2.28) and (6.28.10) we get

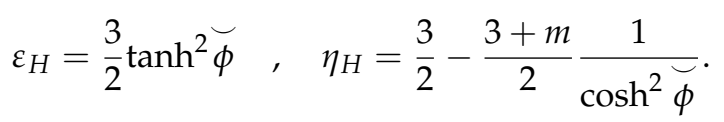

Inserting the expressions (6.28.14) into Equation (4.20) leads to

$$
\delta_{n s}=3-\frac{3-m}{\cosh ^{2} \breve{\phi}} \quad, \quad n_{T}=-3 \tanh ^{2} \breve{\phi} \quad, \quad r=24 \tanh ^{2} \breve{\phi} .
$$

The first and last of these expressions give

$$
r=24 \frac{\delta_{n s}-m}{3-m},
$$

which requires that $0<m<\delta_{n s}$. Solving Equation (6.28.16) with respect to $m$ gives

$$
m=\frac{3\left(8 \delta_{n s}-r\right)}{24-r} \approx \delta_{n s}-\frac{r}{8} .
$$

Inserting $\delta_{n s}=0.032$ and $r=0.05$ we get $m=0.026$. Hence we can approximate $\delta_{n s}$ by

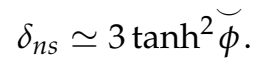

The number of e-folds is

$$
N=\frac{1}{M_{P}^{2}} \int_{\phi_{f}}^{\phi} \frac{V}{V^{\prime}} d \phi .
$$

With the potential (6.28.13) we get

$$
\frac{V^{\prime}}{V}=2 \tanh \left(\frac{3}{m}+\frac{1}{1+\cosh ^{2} \stackrel{\phi}{~}}\right) .
$$

Since the Planck data and Equation (6.28.17) show that $m<0.04$, the first term in the parenthesis is at least 150 times larger than the latter. Hence we can with good approximation neglect the last term during the slow roll era. This gives

$$
N \simeq \frac{m}{6 M_{P}^{2}} \int_{\phi_{f}}^{\phi} \operatorname{coth} \breve{\phi} d \phi=\frac{2}{m} \ln \frac{\sinh \breve{\phi}}{\sinh \breve{\phi}_{f}},
$$

or

$$
\sinh \breve{\varphi} \simeq \sinh \breve{\varphi}_{f} e_{(m / 2) N} .
$$

The value of the inflaton field at the end of inflation is given by $\varepsilon_{H}\left(\breve{\phi}_{f}\right)=1$ which leads to $\tanh ^{2} \breve{\phi}_{f}=2 / 3$. Inserting this into Equation (6.28.22) gives

$$
\tanh ^{2} \breve{\varphi} \simeq \frac{1}{1+(5 / 4) e^{-m N}} .
$$

Hence the optical parameters as given in Equations (6.28.15) and (6.28.18) are

$$
\delta_{n s}=-n_{T}=r / 8=\frac{3}{1+(5 / 4) e^{m N}} .
$$


It follows that the number of e-folds is given in terms of $\delta_{n s}$ as

$$
N \simeq \frac{1}{m} \ln \left(\frac{12}{5 \delta_{n s}}\right)
$$

Inserting the expression (6.28.17) for $m$ we obtain

$$
N \simeq \frac{8}{8 \delta_{n s}-r} \ln \left(\frac{12}{5 \delta_{n s}}\right)
$$

With $\delta_{n s}=0.032, r=0.05$ the number of e-folds in GCG- inflation is $N=168$. Since the number of e-folds is usually restricted to $50<N<60$ it is concluded that a universe dominated by generalized Chaplygin gas is not a suitable model of the inflationary era.

\subsection{Axion Monodromy Inflation}

We shall consider the axion monodromy inflation model with a potential [169]

$$
V=a_{1} \hat{\phi}+a_{2} \cos \left(\frac{\hat{\phi}}{f}+\delta\right) .
$$

A more general potential with $\hat{\phi}$ replaced by $\hat{\phi}^{p}$ in the first term has been considered by Minor and Kaplinghat [47]. With the potential (6.29.1) the potential slow roll parameters are

$$
\varepsilon=\frac{1}{2}\left(\frac{1-x \sin \theta}{\hat{\phi}+A \cos \theta}\right)^{2}, \quad \eta=-\frac{x^{2}}{A} \frac{\cos \theta}{(\hat{\phi}+A \cos \theta)} \quad, \quad \xi=-\frac{x}{A} \sqrt{2 \varepsilon} \eta \tan \theta
$$

where $A=a_{2} / a_{1}, \theta=\hat{\phi} / f+\delta, x=A / f$. The BICEP2/Planck data imply that $\varepsilon \simeq 0.007, \eta \simeq 0.006$, $\xi=0.005$. It follows from the expressions (6.29.2) that

$$
\varepsilon=\frac{A^{2}}{2 x^{4}}\left(\frac{1-x \sin \theta}{\cos \theta}\right)^{2} \eta^{2} \quad, \quad \xi=\frac{1}{x} \frac{(1-x \sin \theta) \sin \theta}{\cos ^{2} \theta} \eta^{2}=\frac{2 x^{3}}{A^{2}} \frac{\sin \theta}{1-x \sin \theta} \varepsilon .
$$

From the last of the Equation (6.29.2) and the first of Equation (6.29.3) we get

$$
A=-\frac{\sqrt{2 \varepsilon} \eta}{\xi\left(1+\frac{\xi}{\eta^{2}} \cot ^{2} \theta\right) \cos \theta} \quad, \quad x=\frac{1}{\left(1+\frac{\xi}{\eta^{2}} \cot ^{2} \theta\right) \sin \theta} \quad, \quad f=-\frac{\sqrt{2 \varepsilon} \eta}{\xi} \tan \theta .
$$

It follows from Equation (6.29.2) that

$$
\hat{\phi}=\sqrt{2 \varepsilon} \frac{1+f^{2} \eta}{2 \varepsilon+f^{2} \zeta} .
$$

or

$$
f^{2}=\sqrt{2 \varepsilon} \frac{1-\hat{\phi} \sqrt{2 \varepsilon}}{\hat{\phi} \xi-\eta \sqrt{2 \varepsilon}},
$$

which requires $\hat{\phi}<\phi_{\max }=1 / \sqrt{2 \varepsilon}$. With $\varepsilon=0.007$ this gives $\hat{\phi}<8$.3. For illustration we choose $\hat{\phi}=7$ since the value $\hat{\phi}=10$ chosen by Kobayashi et al. [169] is not allowed. From Equations (6.29.6) and (6.29.4) we then get $\theta=1.67, f=1.4, A=1, x=0.7$. Hence $\sin \theta=0.99, \cos \theta=-0.10$ and $\delta=-1.9$. 
We shall only give a rough estimate of the upper limit of the value of $N$ given the above values of the slow roll parameters and the constants. Since $\left|A \hat{\phi}^{-1} \cos \theta\right|=0.02<<1$ we shall neglect the term $A \hat{\phi}^{-1} \cos \theta$ in $\varepsilon$ when calculating $N$. This gives

$$
N \simeq \frac{\phi^{2}-\phi_{f}^{2}}{2(1-x \sin \theta)}<\frac{\phi^{2}}{2(1-x \sin \theta)} .
$$

Let us assume that $\hat{\phi}=p \hat{\phi}_{\max }=p / \sqrt{2 \varepsilon}$ where $0<p<1$. Inserting this into Equation (4.29.6) we get

$$
f^{2}=\frac{(1-p) 2 \varepsilon}{p \xi-2 \varepsilon \eta}
$$

From this and Equation (6.29.4) we obtain

$$
x \sin \theta=1 /\left[1+\left(\xi / \eta^{2}\right) \cot ^{2} \theta\right]=\frac{(1-p) \xi}{\xi-2 \varepsilon \eta} .
$$

Inserting Equation (6.29.9) and $\hat{\phi}=p / \sqrt{2 \varepsilon}$ into the inequality (6.29.7) gives

$$
N<\frac{\xi-2 \varepsilon \eta}{p \xi-2 \varepsilon \eta} \frac{p^{2}}{4 \varepsilon} \approx \frac{p}{4 \varepsilon} .
$$

With $r=16 \varepsilon$ we get

$$
r<\frac{4 p}{N}
$$

Inserting $p=0.5$ and $N=50$ gives $r<0.04$ in agreement with the observational data.

\subsection{Intermediate Inflation}

Intermediate inflation models were introduced by J. D. Barrow in 1990 [170,171]. I will here follow the presentations of A. Mohammadi et al. [172] and Rezazadeh et al. [173]. In both of these papers intermediate models with a no-canonical scalar field were considered. In the present review I will describe the corresponding class of models with a canonical scalar field by specializing to the case $n=1, \quad F_{0}=1$ in [172] or equivalently to $\alpha=1$ in [173].

The point of departure is Barrow's assumption for the time-dependency of the scale factor,

$$
a(t)=a_{0} e^{A \hat{t}^{\alpha}} \quad, \quad 0<\alpha<1 .
$$

Here $\mathrm{A}$ is a positive dimensionless constant, $a_{0}$ is the value of the scale factor at $t=0$ and $\hat{t}=t / t_{P}$ where $t_{P}$ is the Planck time. It turns out that the constant $A$ does not appear in the expressions for the optical parameters. In cold inflation its value has no consequence for the predictions of this class of models. Hence, without loss of generality we can put $A=1$.

The models are called 'intermediate' because the expansion is faster than power law expansion and slower than the exponential expansion of the de Sitter spacetime, which has $\alpha=1$. The Hubble parameter, its rate of change and and its second derivative are

$$
H=M_{P} \alpha \hat{t}^{\alpha-1}, \quad \dot{H}=-M_{P}^{2} \alpha(1-\alpha) \hat{t}^{\alpha-2}, \quad \ddot{H}=M_{P}^{3} \alpha(1-\alpha)(2-\alpha) \hat{t}^{\alpha-3} .
$$

From Equations (2.1) and (2.6) together with the identity $\ddot{a}=H^{2}+\dot{H}$, we get

$$
\rho=3 M_{P}^{4} \alpha^{2} \hat{t}^{2(\alpha-1)} \quad, \quad p=M_{P}^{4} \alpha \hat{t}^{\alpha-2}\left[2(1-\alpha)-3 \alpha \hat{t}^{\alpha}\right] .
$$

Equation (2.1) implies

$$
\rho+p=\dot{\phi}^{2}
$$


Inserting (6.30.3) and integrating with the initial condition $\phi(0)=0$ gives

$$
\hat{\phi}(t)=2 \sqrt{2 \frac{1-\alpha}{\alpha}} \hat{t}^{\alpha / 2}
$$

Equation (2.1) also gives

$$
V=\frac{1}{2}(\rho-p)
$$

Inserting (6.30.3) we get

$$
V(t)=M_{P}^{4} \alpha \hat{t}^{\alpha-2}\left[3 \alpha \hat{t}^{\alpha}-2(1-\alpha)\right] .
$$

Using Equation (6.30.5) we obtain the potential as a function of the inflaton field

$$
V(\phi)=M_{P}^{4} \alpha\left[\frac{\alpha}{2(1-\alpha)}\right]^{\frac{\alpha-2}{\alpha}}\left(\frac{\hat{\phi}}{2}\right)^{\frac{2(\alpha-2)}{\alpha}}\left[\frac{3 \alpha^{2}}{2(\alpha-2)}\left(\frac{\hat{\phi}}{2}\right)^{2}-2(1-\alpha)\right]
$$

For this class of models the spectral parameters are most easily calculated from the Hubble slow roll parameters (3.12) using Equation (6.30.2). This gives

$$
\varepsilon_{H}=\frac{1-\alpha}{\alpha} \hat{t}^{-\alpha}, \quad \eta_{H}=\frac{2-\alpha}{2(1-\alpha)} \varepsilon_{H} .
$$

Note that the slow roll parameter $\varepsilon_{H}$ is a decreasing function of time for intermediate inflation. This means that there is no natural end of the inflationary era in this class of models, and one just postulates that the inflationary era lasts for a suitable number of e-folds, say $50<N<60$. In this class of models one defines instead the beginning of the inflationary era by the condition $\varepsilon_{H}\left(t_{i}\right)=1$, and evaluates the spectral parameters at the end of the inflationary era. However, Rezazadeh et al. [173] have invoked a non-canonical scalar field in order to solve the exit problem for intermediate inflation.

Using Equation (6.30.5) the slow roll parameter $\varepsilon_{H}$ is expressed in terms of the inflaton field as

$$
\varepsilon_{H}=8\left(\frac{1-\alpha}{\alpha}\right)^{2} \frac{1}{\hat{\phi}^{2}} .
$$

It follows from Equations (3.46) and (6.30.1) that the number of e-folds is

$$
N=\hat{t}^{\alpha}-\hat{t}_{i}^{\alpha},
$$

where $t_{i}$ is the initial point of time of the inflationary era, defined by $\varepsilon_{H}\left(t_{i}\right)=1$ giving

$$
\hat{t}_{i}^{\alpha}=\frac{1-\alpha}{\alpha} .
$$

Inserting this into Equation (6.30.11) gives for the point of time when the slow roll parameters are evaluated

$$
\hat{t}^{\alpha}=\frac{N \alpha+1-\alpha}{\alpha},
$$

Giving

$$
\varepsilon_{H}=\frac{1-\alpha}{N \alpha+1-\alpha} \quad, \quad \eta_{H}=\frac{2-\alpha}{2(N \alpha+1-\alpha)} .
$$


The spectral parameters $\delta_{n s}, n_{T}$ and $r$ are expressed in terms of the Hubble slow roll parameters in Equation (4.20). Inserting the expressions (6.30.14) we obtain

$$
\delta_{n s}=\frac{2-3 \alpha}{N \alpha+1-\alpha} \quad, \quad n_{T}=-\frac{2(1-\alpha)}{N \alpha+1-\alpha} \quad, \quad r=\frac{16(1-\alpha)}{N \alpha+1-\alpha} .
$$

Note that the curvature spectrum is scale independent, corresponding to $\delta_{n s}=0$, for $\alpha=2 / 3$. The expression for $n_{s}$ corrects an error in Mohammadi et al. [172]. For these model the $r, \delta_{n s}-$ relationship is

$$
r=\frac{16(1-\alpha)}{2-3 \alpha} \delta_{n s} .
$$

The constant $\alpha$ can be expressed in terms of $N$ and $\delta_{n s}$ as

$$
\alpha=\frac{2-\delta_{n s}}{3+(N-1) \delta_{n s}} \approx \frac{2}{3+N \delta_{n s}} .
$$

Inserting the last expression into Equation (6.30.16) gives

$$
r \approx \frac{1+N \delta_{n s}}{N \delta_{n s}} 8 \delta_{n s}>8 \delta_{n s} .
$$

With the Planck values $\delta_{n s}=0.032$ and $N=60$ we get $r>0.256$. This value of $r$ is larger than permitted by the Planck observations. However the more general models with non-canonical inflaton fields studied by Mohammadi et al. [172] and Rezazadeh et al. [173] contain an additional adjustable parameter in the expressions for the observable parameters, making agreement with observational data possible. A class of intermediate inflationary models with a variable sound velocity have recently been investigated by N. Nazavari et al. [174]. In these models one may obtain agreement with the Planck data. Below we shall consider warm intermediate inflation models, and they lead naturally to a suppression of the curvature perturbation, giving a small value of $r$.

\section{Brane-Intermediate Inflation}

S. del Campo and R. Herrera [175] have investigated observable consequences of intermediate inflation on the brane. The scale factor is given in Equation (6.30.1), and the Hubble parameter and its derivatives in Equation (6.30.2). Inserting these expressions into Equation (6.16.16) gives

$$
\dot{\phi}=\left(\frac{2 \lambda}{3}\right)^{1 / 4} \sqrt{\frac{1-\alpha}{\hat{t}}} .
$$

Integrating with $\phi(0)=0$ leads to

$$
\phi=2\left(\frac{2 \lambda}{3}\right)^{1 / 4} \sqrt{(1-\alpha) \hat{t}}
$$

From the expressions (6.30.2) and Equation (6.16.19) we get

$$
V(t)=\frac{1}{\hat{t}} \sqrt{\frac{\lambda}{6}}\left[6 \alpha \hat{t}^{\alpha}-(1-\alpha)\right] .
$$

From Equations (6.30.19) and (6.30.20) the potential is

$$
\kappa V(\phi)=\frac{\lambda}{\hat{\phi}^{2}}\left\{6 \alpha\left[\frac{4}{3}(1-\alpha)\right]^{1-\alpha}\left(\frac{1}{6 \lambda}\right)^{\alpha / 2} \hat{\phi}^{2 \alpha}-\frac{4}{3}(1-\alpha)^{2}\right\} .
$$


The brane tension is usually assumed to be very small in Planck units, so in the strong field case the first term inside the brackets dominates. Hence we can approximate the potential with

$$
V(\phi)=B \hat{\phi}^{-2(1-\alpha)} \quad, \quad B=6 \alpha \lambda\left[\frac{4}{3}(1-\alpha)\right]^{1-\alpha}\left(\frac{1}{6 \lambda}\right)^{\alpha / 2},
$$

which has the same form as in polynomial inflation with $p=2(\alpha-1)$. However the predicted values of the spectral parameters are different from those of polynomial standard inflation since the dynamical equations are different in brane-inflation.

The Hubble slow roll parameters as functions of time are the same as in standard inflation, and are given in Equation (6.30.9). Combining these expressions with Equation (6.30.20) gives

$$
\varepsilon_{H}=\frac{1-\alpha}{\alpha}\left[4(1-\alpha) \sqrt{\frac{2 \lambda}{3}}\right]^{\alpha} \hat{\phi}^{-2 \alpha}, \quad \eta_{H}=\frac{2-\alpha}{2 \alpha}\left[4(1-\alpha) \sqrt{\frac{2 \lambda}{3}}\right]^{\alpha} \hat{\phi}^{-2 \alpha} .
$$

The inflaton field at the end of the inflationary era is given by $\eta_{H}\left(\phi_{f}\right)=1$, giving

$$
\phi_{f}^{2 \alpha}=\frac{2-\alpha}{2 \alpha}\left[4(1-\alpha) \sqrt{\frac{2 \lambda}{3}}\right]^{\alpha} .
$$

The number of e-folds during the slow roll era is found by combining Equations (6.30.11), (6.30.19), (6.30.23) and (6.30.24), giving

$$
\varepsilon_{H}=\frac{2(1-\alpha)}{2 N \alpha+2-\alpha} \quad, \quad \eta_{H}=\frac{2-\alpha}{2 N \alpha+2-\alpha} .
$$

In spite of the different time dependence of the inflaton field for the standard intermediate inflation and the brane-intermediate inflation the expressions for the slow roll parameters are identical. The reason is that they depend only upon the time dependence of the scale factor.

However the expressions (4.20) are modified, so Equations (6.30.15)-(6.30.17) are not valid for brane-intermediate inflation. Del Campo and Herrera [175] have shown that

$$
\delta_{n s}=2\left(3 \varepsilon_{H}-\eta_{H}\right) .
$$

Inserting the expressions (6.30.25) gives

$$
\delta_{n s}=\frac{2(4-5 \alpha)}{2 N \alpha+2-\alpha}
$$

or

$$
\alpha=\frac{2\left(4-\delta_{n s}\right)}{10+2 N \delta_{n s}-\delta_{n s}} \approx \frac{4}{5+N \delta_{n s}} .
$$

Inserting $\delta_{n s}=0.032$ and $50<N<60$ gives $0.58<\alpha<0.61$. This is a little smaller that the values $2 / 3<\alpha<4 / 5$ del Campo and Herrera arrived at with the observational data up to 2009 .

Del Campo and Herrera found that the running of the scalar index is

$$
\alpha_{S}=-\frac{\alpha}{3-4 \alpha} \delta_{n s}^{2}
$$

giving $-0.0011<\alpha_{S}<-0.0009$ in agreement with observational data. They found that the tensor-scalar ratio is given by

$$
r=\frac{8(1-\alpha)}{\alpha^{2}} F\left(n_{S}\right) \hat{t}^{1-2 \alpha} \quad, \quad F\left(n_{S}\right)=\left[\sqrt{1+n_{S}^{2}}-n_{S}^{2} \operatorname{Arsinh}\left(1 / n_{S}\right)\right]^{-1} .
$$


Inserting the expressions (6.30.9) into Equation (6.30.26) leads to

$$
\delta_{n s}=\frac{4-5 \alpha}{\alpha} \hat{t}^{-\alpha} .
$$

It follows from the last two equations that the $r, \delta_{n s}-$ relation is

$$
r=\frac{8(1-\alpha)}{\alpha^{1 / \alpha}(4-5 \alpha)^{2-1 / \alpha}} \sqrt{6 \lambda} F\left(n_{S}\right) \delta_{n s}^{2-1 / \alpha} .
$$

With $n_{S} \approx 1$ we get $F\left(n_{S}\right) \approx 2$. From the above result a proper choice of $\alpha$ is $\alpha=0.6$. Furthermore we insert the favored Planck value $\delta_{n s}=0.032$. Recently J. R. Gott III and W. N. Colley [46] have shown that from the best available data it follow that $0<r<0.04$. Inserting these values into Equation (6.30.32) gives $A>0.01$.

\subsection{Constant- Roll Inflation}

H. Motohashy, A. A. Starobinsky and J. Yokoyama [107,176], F. Cicciarella, J. Mabillard, J and M. Pieroni [177] and A. Karam et al. [178] have recently considered a class of inflationary models with constant rate of roll. They defined the 'rate of roll' as $\ddot{\phi} / H \dot{\phi}$. According to Equation (3.16) this is the same as minus the Hubble parameter $\eta_{H}$. Hence the general condition for constant rate of slow roll inflation may be written

$$
\eta_{H}=3+\alpha=-2 \beta=3 \lambda \quad \text { or } \quad \ddot{\phi}=-3 \lambda H \dot{\phi},
$$

where $\alpha, \beta$ and $\lambda$ are constants, $\alpha$ is used in [107], $\beta$ in [171] and $\lambda$ in [172]. It follows from Equations (2.5) and (3.16) that

$$
V^{\prime}=\left(\eta_{H}-3\right) H \phi^{\prime}=\alpha H \dot{\phi} .
$$

Hence, the case $\eta_{H}=3$ or $\alpha=0, \beta=-3 / 2$ and $\lambda=1$ implies that $V(\phi)=$ constant, i.e., it represents a flat potential. We shall first consider this case.

With $\eta_{H}=3$ Equation (3.7) takes the form

$$
2 M_{P}^{2} H_{, \phi \phi}-3 H=0 .
$$

The general solution of this equation is

$$
H(\phi)=A \cosh \left(\sqrt{\frac{3}{2}} \hat{\phi}\right)+B \sinh \left(\sqrt{\frac{3}{2}} \hat{\phi}\right) .
$$

Differentiating Equation (6.31.4) and using Equation (2.14) we have

$$
V=M_{P}^{2}\left(3 H^{2}-2 M_{P}^{2} H^{\prime 2}\right)=3\left(A^{2}-B^{2}\right) M_{P}^{2},
$$

showing that $V$ is indeed constant. In this case Equation (3.12) takes the form

$$
\ddot{H}+6 H \dot{H}=0 .
$$

Integration gives

$$
\dot{H}+3 H^{2}=K_{1} .
$$


where $K_{1}$ is an integration constant. It follows from Equation (2.12) that $\dot{H}<0$. Integration of Equation (6.31.7) then gives

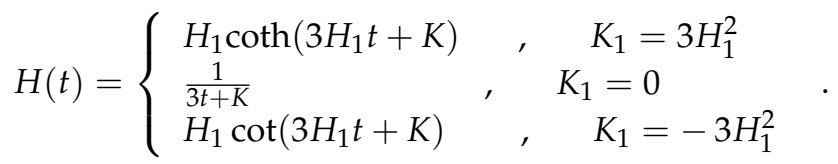

where $K$ is a new integration constant. Equation (3.16) now reduces to

$$
\ddot{\phi}+3 H \dot{\phi}=0 .
$$

Using that $H=\dot{a} / a$ and integrating gives

$$
\dot{\phi}=K_{2} a^{-3} \text {. }
$$

where $K_{2}$ is an integration constant.

Integration of Equation (6.31.8) with $K_{1}>0$ and $a(0)=0$ leads to

$$
a(t)=a_{1} \sinh _{1 / 3}\left(3 H_{1} t\right) .
$$

where $a_{1}$ is an integration constant. Inserting this into Equation (4.31.10), determining the integration constants from Equation (2.12), and assuming that the inflaton field decreases with time gives

$$
\dot{\phi}=-\frac{\sqrt{6} M_{P} H_{1}}{\sinh \left(3 H_{1} t\right)} .
$$

New integration with $\lim _{t \rightarrow \infty} \phi(t)=0$ gives

$$
\phi=\sqrt{\frac{2}{3}} M_{P} \ln \left[\operatorname{coth}\left(\frac{3}{2} H_{1} t\right)\right] .
$$

Differentiating Equation (6.31.8) gives

$$
\dot{H}=-\frac{3 H_{1}^{2}}{\sinh ^{2}\left(3 H_{1} t\right)} \quad, \quad \ddot{H}=-18 H_{1}^{3} \frac{\cosh \left(3 H_{1} t\right)}{\sinh ^{3}\left(3 H_{1} t\right)} .
$$

Inserting Equations (6.31.12) and (6.31.14) into Equation (2.12) gives $K_{1}=\sqrt{6} M_{P} H_{1}$. Equation (2.2) then gives

$$
V=3 M_{P}^{2} H^{2}-(1 / 2) \dot{\phi}^{2}=3 M_{P}^{2} H_{1}^{2},
$$

which is constant. Comparing with Equation (6.31.5) we get $H_{0}^{2}=A^{2}-B^{2}$. The Hubble slow roll parameters,

$$
\varepsilon_{H}=-\frac{\dot{H}}{H^{2}}=\frac{3}{\cosh ^{2}\left(3 H_{1} t\right)} \quad, \quad \eta_{H}=-\frac{1}{2} \frac{\ddot{H}}{H \dot{H}}=3 .
$$

The scalar spectral index is small only at a certain point of time, $t_{1}$, when $\cosh ^{2}\left(3 H_{1} t\right) \approx 2$, so this is not a realistic inflationary model.

We shall now consider the corresponding models with $\eta_{H} \neq 3$, i.e., $\alpha \neq 0$. The case $\eta_{H}=0$ i.e., $\alpha=-3$ corresponds corresponds to the scale invariant case which is in conflict with observation, so this case will not be considered here. Equation (3.7) now takes the form

$$
M_{P}^{2} H_{\phi \phi}+\beta H=0 .
$$


The general solution is

$$
H(\phi)=\left\{\begin{array}{ll}
A \cos (\sqrt{\beta} \hat{\phi})+B \sin (\sqrt{\beta} \hat{\phi}) & \quad \beta>0 \\
A \cosh (\sqrt{-\beta} \hat{\phi})+B \sinh (\sqrt{-\beta} \hat{\phi}), & \beta<0
\end{array} .\right.
$$

Equation (6.31.5) is now generalized to

$$
V(\phi)=\left\{\begin{array}{lll}
M_{P}^{2}\left[3\left(A^{2}+B^{2}\right)-(3+2 \beta)(A \sin \sqrt{\beta} \hat{\phi}-B \cos \sqrt{\beta} \hat{\phi})^{2}\right] & , \quad \beta>0 \\
M_{P}^{2}\left[3\left(A^{2}-B^{2}\right)+(3+2 \beta)(A \sinh \sqrt{-\beta} \hat{\phi}+B \cosh \sqrt{-\beta} \hat{\phi})^{2}\right], & \beta<0
\end{array} .\right.
$$

Motohashy, Starobinsky and Yokoyama [107] have considered 3 special cases.

(1) $\quad A=B=M$ giving

$$
\begin{aligned}
& H(\phi)=\left\{\begin{array}{ll}
M \sqrt{2} \sin (\sqrt{\beta} \hat{\phi}+\pi / 4), & \beta>0 \\
M e^{\sqrt{-\beta} \hat{\phi}} & , \quad \beta<0
\end{array},\right. \\
& V(\phi)=\left\{\begin{array}{lc}
2 M_{P}^{2} M^{2}\left[3-(3+2 \beta) \cos ^{2}(\sqrt{\beta} \hat{\phi}+\pi / 4)\right], & \beta>0 \\
(3+2 \beta) M_{P}^{2} M^{2} e^{\sqrt{-2 \beta}} \hat{\phi} & \beta<0
\end{array} .\right.
\end{aligned}
$$

In the case $\beta<0$ a positive potential requires $\beta>-3 / 2$.

For $-3 / 2<\beta<0$ the potential is recognized as that of power law inflation. Comparing with Equation (6.4.1) we have $\lambda^{2}=-4 \beta$. From Equations (6.4.7) and (6.4.8) we then get $\varepsilon=-2 \beta, \delta_{n s}=-4 \beta, r=-32 \beta$ and hence $r=8 \delta_{n s}$. This value of $r$ is higher than allowed by the Planck observations.

(2) $B=0, A=M$ giving

$$
\begin{gathered}
H(\phi)=\left\{\begin{array}{ll}
M \cos (\sqrt{\beta} \hat{\phi}) & , \quad \beta>0 \\
M \cosh (\sqrt{-\beta} \hat{\phi}), & \beta<0
\end{array},\right. \\
V(\phi)=\left\{\begin{array}{ll}
M_{P}^{2} A^{2}\left[3+(3+2 \beta) \sin ^{2}\left(\sqrt{\beta} \hat{\phi}^{2}\right)\right], \quad \beta>0 \\
M_{P}^{2} A^{2}\left[3-(3+2 \beta) \sin ^{2}\left(\sqrt{-\beta} \hat{\phi}^{2}\right)\right], \quad \beta<0
\end{array} .\right.
\end{gathered}
$$

(3) $\quad A=0, B=M$ giving

$$
\begin{aligned}
& H(\phi)=\left\{\begin{array}{ll}
M \sin (\sqrt{\beta} \hat{\phi}) & , \quad \beta>0 \\
M \sinh (\sqrt{-\beta} \hat{\phi}) & \quad \beta<0
\end{array},\right. \\
& V(\phi)=\left\{\begin{array}{ll}
M_{P}^{2} M^{2}\left[-3+(3+2 \beta) \cos ^{2}(\sqrt{\beta} \hat{\phi})\right], & \beta>0 \\
M_{P}^{2} M^{2}\left[3-(3+2 \beta) \cosh ^{2}(\sqrt{-\beta} \hat{\phi})\right], & \beta<0
\end{array} .\right.
\end{aligned}
$$

It may be noted that the expressions (6.31.23) and (6.31.25) for $\beta>0$ are similar to those for natural inflation as given in Equation (6.5.1). The predictions of the model with $B=0$ has been compared with the Planck data by Motohashi and Starobinsky [176].

In this case Equation (3.12) takes the form

$$
\ddot{H}-4 \beta H \dot{H}=0 .
$$

Hence, the generalization of Equation (4.31.7) is

$$
\dot{H}-2 \beta H^{2}=K_{1} \text {. }
$$


Integrating this equation we have to consider some cases.

1. Let us first consider the case $K_{1}=0$. Integration of Equation (6.31.27) then gives

$$
H=\frac{1}{K-2 \beta t} \text {. }
$$

Then the special case $\beta=0$ gives the constant Hubble parameter of a universe with exponential expansion such as the De Sitter spacetime dominated by a Lorentz Invariant Vacuum Energy (LIVE) with a constant density that may be represented by a cosmological constant.

A flat universe FLRW-universe dominated by a perfect fluid with equation of state $p=w \rho$ has a Hubble parameter

$$
H=\frac{2}{3(1+w) t} .
$$

Hence, putting $K=0$ in Equation (6.33.28) gives the Hubble parameter of a flat FLRW-universe dominated by a perfect fluid with equation of state parameter

$$
w=-\left(1+\frac{4}{3} \beta\right)
$$

The condition $-1 \leq w \leq 1$ interpolating between LIVE and a Zel'dovich fluid restricts $\beta$ to the interval $-3 / 2 \leq \beta \leq 0$.

2. Next we consider the case $K_{1}=-2 \beta H_{1}^{2}$. Then Equation (6.31.27) takes the form

$$
\dot{H}=-2 \beta\left(H_{1}^{2}-H^{2}\right),
$$

Since $\dot{H}$ is negative $\beta<0$ requires $H>H_{1}$, and $\beta>0$ requires $H<H_{1}$. Solving Equation (6.31.31) with these requirements in mind leads to

$$
H(t)=\left\{\begin{array}{ll}
H_{1} \tanh \left(2 \beta H_{1} t+K\right) \quad, \quad \beta>0 \\
H_{1} \operatorname{coth}\left(-2 \beta H_{1} t+K\right) \quad, \quad \beta<0
\end{array} .\right.
$$

3. Finally we have the case $K_{1}=2 \beta H_{1}^{2}$. Then

$$
\dot{H}=2 \beta\left(H_{1}^{2}+H^{2}\right),
$$

which requires $\beta<0$. The solution of Equation (6.31.33) is

$$
H(t)=H_{1} \cot \left(-2 \beta H_{1} t+K\right) .
$$

Integrating the expressions for the Hubble parameter we get the time evolution of the scale factor. We shall only consider the cases 2 and 3. From Equation (6.31.32) with $K=0$ we obtain

$$
a(t)=\left\{\begin{array}{ll}
a_{1} \cosh ^{-1 / \beta}\left(2 \beta H_{1} t\right), & \beta>0 \\
a_{2} \sinh ^{-1 / \beta}\left(-2 \beta H_{1} t\right), & \beta<0
\end{array},\right.
$$

It was noted by Motohashi et al. [176] that also in this case the expression for the scale factor in the case $\beta<0$ corresponds to that for a flat FLRW model with a cosmological constant filled with a perfect fluid with equation of state $p=w \rho$, where $\beta=-3(1+w) / 4$. Hence the case $\beta=-3 / 4$ corresponds to the standard $\Lambda \mathrm{CDM}$ universe model.

From Equation (6.31.34) with $K=0$ we get

$$
a(t)=a_{3} \sin \left(-2 \beta H_{1} t\right) .
$$


The cases 2 and 3 behave in a similar manner for small values of $-\beta H_{1} t$. Hence from now on we shall only consider the case 2 .

Equation (3.13) then takes the form

$$
\ddot{\phi}-2 \beta H \dot{\phi}=0,
$$

giving

$$
\dot{\phi}=K_{1} a^{2 \beta}
$$

For the case 2 we now get

$$
\dot{\phi}=\left\{\begin{array}{ll}
-\frac{2 M_{P} H_{1} \sqrt{\beta}}{\cosh \left(2 \beta H_{1} t\right)}, & \beta>0 \\
-\frac{2 M_{P} H_{1} \sqrt{-\beta}}{\sinh \left(2 \beta H_{1} t\right)}, & \beta<0
\end{array},\right.
$$

while the case 3 leads to

$$
\dot{\phi}=-\frac{2 M_{P} H_{1} \sqrt{-\beta}}{\sin \left(2 \beta H_{1} t\right)} .
$$

Integration of Equation (6.31.39) gives

$$
\phi(t)=\left\{\begin{array}{ll}
2 M_{P} H_{1} \sqrt{1 / \beta} \arctan \left(e^{-2 \beta H_{1} t}\right) & , \quad \beta>0 \\
M_{P} H_{1} \sqrt{-1 / \beta} \ln \left[\operatorname{coth}\left(\beta H_{1} t\right)\right] & \quad \beta<0
\end{array},\right.
$$

and of Equation (6.31.40),

$$
\phi(t)=M_{P} H_{1} \sqrt{-1 / \beta} \ln \left[\cot \left(\beta H_{1} t\right)\right] .
$$

Differentiating Equation (6.31.32) gives

$$
\dot{H}=\left\{\begin{array}{l}
\frac{2 \beta H_{1}^{2}}{\cosh ^{2}\left(2 \beta H_{1} t\right)} \\
-\frac{2 \beta H_{1}^{2}}{\sinh ^{2}\left(2 \beta H_{1} t\right)}
\end{array} \quad, \quad \ddot{H}= \begin{cases}8 \beta^{2} H_{1}^{3} \frac{\sinh \left(2 \beta H_{1} t\right)}{\cosh ^{3}\left(2 \beta H_{1} t\right)}, & \beta>0 \\
-8 \beta^{2} H_{1}^{3} \frac{\cosh ^{2}\left(2 \beta H_{1} t\right)}{\sinh ^{3}\left(2 \beta H_{1} t\right)}, & \beta<0\end{cases}\right.
$$

The time dependency of the inflaton field in the case 2 is

$$
V(t)=3 M_{P}^{2} H^{2}-\frac{1}{2} \dot{\phi}^{2}=M_{P}^{2} H_{1}^{2}\left\{\begin{array}{ll}
3-\frac{3+2 \beta}{\cosh ^{2}\left(2 \beta H_{1} t\right)}, & \beta>0 \\
3+\frac{3+2 \beta}{\sinh ^{2}\left(2 \beta H_{1} t\right)} \quad, \quad \beta<0
\end{array} .\right.
$$

Inserting the expressions (6.32.41) into (6.31.44) gives the inflaton potential for the case 2

$$
V(\phi)=M_{P}^{2} H_{1}^{2}\left\{\begin{array}{ll}
3 \cos ^{2}\left(\frac{\sqrt{\beta}}{M_{P} H_{1}} \phi\right)-\beta \sin ^{2}\left(\frac{\sqrt{\beta}}{M_{P} H_{1}} \phi\right) & , \quad \beta>0 \\
3 \cosh ^{2}\left(\frac{\sqrt{-\beta}}{M_{P} H_{1}} \phi\right)+\beta \sinh ^{2}\left(\frac{\sqrt{-\beta}}{M_{P} H_{1}} \phi\right) & , \quad \beta<0
\end{array} .\right.
$$

The Hubble slow roll parameters for this case are

$$
\varepsilon_{H}=\left\{\begin{array}{l}
\frac{2 \beta}{\sinh ^{2}\left(2 \beta H_{1} t\right)} \\
-\frac{\beta}{\cosh ^{2}\left(\beta H_{1} t\right)}
\end{array} \quad, \quad \eta_{H}=\left\{\begin{array}{ll}
\beta, & \beta>0 \\
-\beta, & \beta<0
\end{array} .\right.\right.
$$


The tilt of the scalar spectral index and the tensor-to-scalar-ratio are

$$
\begin{gathered}
\delta_{n s}=2\left(2 \varepsilon_{H}-\eta_{H}\right)=\left\{\begin{array}{ll}
4 \beta \frac{2-\sinh ^{2}\left(2 \beta H_{1} t\right)}{\sinh ^{2}\left(2 \beta H_{1} t\right)} & , \quad \beta>0 \\
-4 \beta \frac{2-\cosh ^{2}\left(2 \beta H_{1} t\right)}{\cosh ^{2}\left(2 \beta H_{1} t\right)}, & \beta<0
\end{array} .\right. \\
r=16 \varepsilon_{H}=\left\{\begin{array}{ll}
\frac{32 \beta}{\sinh ^{2}\left(2 \beta H_{1} t\right)}, & \beta>0 \\
-\frac{32 \beta}{\cosh ^{2}\left(2 \beta H_{1} t\right)}, & \beta<0
\end{array} .\right.
\end{gathered}
$$

In all cases the $\delta_{n s}, r-$ relationship is

$$
\beta=\frac{1}{8}\left(4 \delta_{n s}-r\right) .
$$

The preferred values from the Planck observations, $r=0.05, \delta_{n s}=0.032$ give $\beta=0.005$. Hence the case $\beta>0$ is favored with $\beta \approx 0$. The values $\beta=-3 / 2$ and $\beta=-3$ are not permitted. The observational constraint $r<0.04, \delta_{n s}=0.032$ gives the restriction $\beta>0.005$.

A related class of inflationary models, called 'constant slow-roll inflation', has been considered by Gao and Gong [74]. They are defined by the condition that the slow roll parameter $\eta$ defined in Equation (3.1) is constant, hence

$$
V,_{\phi \phi}-\kappa \eta V=0 .
$$

Integration gives

$$
V(\phi)=\left\{\begin{array}{ll}
A e^{\sqrt{\eta} \hat{\phi}}+B e^{-\sqrt{\eta} \hat{\phi}} & , \quad 0<\eta<1 \\
A+B \phi & , \quad \eta=0 \\
A \cos (\sqrt{-\eta} \hat{\phi})+B \sin (\sqrt{-\eta} \hat{\phi}) & , \quad-1<\eta<0
\end{array} .\right.
$$

These are power law inflation with exponential potential, linear polynomial inflation and natural inflation.

One may use the $\mathrm{N}$-fold formalism to investigate this class of inflationary models. Integrating Equation (5.44), i.e.,

$$
\left[\ln \left(V^{2}, N\right)\right], N=2 \eta
$$

gives

$$
V(N)=\sqrt{A+B e^{2 \eta N},}
$$

where $A$ and $B$ are constants of integration. Integrating Equation (3.58), i.e.,

$$
\varepsilon,_{N}=2 \varepsilon(\eta-2 \varepsilon)
$$

with the boundary condition $\varepsilon(0)=1$ gives

$$
\varepsilon(N)=\frac{\eta e^{2 \eta N}}{\eta+2\left(e^{2 \eta N}-1\right)} .
$$

Hence the tensor-to-scalar ratio is

$$
r=\frac{16 \eta e^{2 \eta N}}{\eta+2\left(e^{2 \eta N}-1\right)} .
$$

The scalar spectral tilt is

$$
\delta_{n s}=-2 \frac{\eta-2-e^{2 \eta N}}{\eta-2+2 e^{2 \eta N}} \eta
$$


The two last equations give

$$
\eta=(1 / 16)\left(3 r-8 \delta_{n s}\right) .
$$

From Equations (6.31.54) and (6.31.56) we get

$$
N=\frac{8}{8 \delta_{n s}-3 r} \ln \frac{16\left(8 \delta_{n s}-r\right)}{\left(32-3 r+8 \delta_{n s}\right) r} \approx \frac{8}{8 \delta_{n s}-3 r} \ln \left[\frac{1}{2}\left(8 \frac{\delta_{n s}}{r}-1\right)\right] .
$$

Inserting $\delta_{n s}=0.032, r=0.05$ gives $\eta=-0.0067$ and $N=54.3$. Hence, among the 3 classes of models in Equation (6.31.49) the one with $\eta<0$ is favored by the observational data. We shall therefore consider this class of models.

It follows from Equation (3.50) that the inflaton field is given in terms of the number of e-folds by

$$
\hat{\phi}=\hat{\phi}_{f}+\int_{0}^{N} \sqrt{2 \varepsilon(N)} d N
$$

Inserting the expression (6.31.54) we find

$$
\hat{\phi}=\frac{1}{\sqrt{-\eta}} \arcsin \left(\sqrt{\frac{2}{2-\eta}} e^{\eta N}\right), \quad \hat{\phi}_{f}=\frac{1}{\sqrt{-\eta}} \arcsin \left(\sqrt{\frac{2}{2-\eta}}\right) .
$$

which is different from Equation (12) of Gao [74]. Inserting the expression (6.31.60) into Equation (6.31.50) and choosing $A=0, B=1-\eta / 2$ gives

$$
V(\phi)=(1-\eta / 2) \sin (\sqrt{-\eta} \hat{\phi}) .
$$

This is the form of the potential of the natural inflationary models.

Cicciarella, Mabillard and Pieroni (CMP) [177] have recently applied the $\beta$ - function formalism to the constant rate of roll inflationary models. It follows from Equations (5.127) and (6.31.1) that the condition for constant rate of roll inflation can be written in terms of the $\beta$ - function as

$$
\frac{1}{2} \beta^{2}-\frac{\beta^{\prime}}{\kappa}=3 \lambda
$$

It follows from Equations (5.128) and (6.31.62) that for these models the scalar spectral index and the tensor-to-scalar ratio are related by

$$
r=4\left(\delta_{n s}+6 \lambda\right)
$$

CMP [177] have integrated Equation (6.31.62) for different cases.

1. De Sitter and chaotic models, $\lambda=0$. In this case there are two solutions.

1A. $\beta(\phi)=0$. Equation (5.112) then gives a constant Hubble parameter, $H=H_{0}$ with exponential expansion, $a \propto e^{H_{0} t}$. This represents the De Sitter spacetime. In this case the condition $\beta\left(\phi_{f}\right)=1$ is never obtained, and therefore there is no end to the slow roll era.

1B. $\beta(\phi)=-2 / \phi$. This is a member of the chaotic class of Binétruy et al. [82] with properties described in the Equations (5.159)-(5.169).

It follows from Equation (6.31.33) that the models $1 \mathrm{~A}$ and $1 \mathrm{~B}$ have $r=4 \delta_{n s}=0.128$ and are ruled out by the BPK-data.

2. Constant $\beta$-function, $\beta^{\prime}=0$. It follows from Equation (6.31.61) that $\lambda>0$ in this case with $\beta(\phi)= \pm \sqrt{6 \lambda}$. This is a member of the power law class of Binétruy et al. [82]. In this case $r>0.128$, so this model is ruled out by the BPK-data. 
3. Fast roll inflation. General case with $\lambda>0$. This class of models will be discussed in Section 6.17. For these models Equation (6.31.63) gives $r>0.128$, so they are ruled out by the BPK-data.

4. Interpolating model with $\lambda<0$. In order to simplify the expression we introduce in this case a constant $\gamma$ by $\lambda=-(2 / 3) \gamma^{2}$. It follows from Equation (6.31.63) that the BPK-data, $\delta_{n s}=0.032, \mathrm{r}<0.04$ require $\lambda<-0.0037$ or $\gamma>0.074$. In this case the solution of Equation (6.31.62) may be written

$$
\beta(\phi)=(2 \gamma / \sqrt{\kappa}) \tan (\gamma \hat{\phi}) .
$$

Hence this model Interpolates between the linear class of models described in Equations (5.150) to (5.157) and the class of chaotic models, respectively in the limits $\phi \rightarrow 0$ and $\hat{\phi} \rightarrow \pi / 2 \gamma$. Inserting the expression (6.31.64) into Equation (5.113) and integrating gives the Hubble parameter

$$
H=H_{1} \cos (\gamma \hat{\phi}),
$$

and inserting the expressions (6.31.64) and (6.31.65) into Equation (5.125) gives the potential

$$
\kappa V(\phi)=V_{0}\left[1-(1-\lambda) \sin ^{2}(\gamma \hat{\phi})\right],
$$

where $V_{0}=V(0)$. Inserting the function (6.31.64) into Equation (5.118) gives the number of e-folds

$$
N=-\frac{1}{2 \gamma^{2}} \ln \frac{\sin (\gamma \hat{\phi})}{\sin \left(\gamma \hat{\phi}_{f}\right)} .
$$

It follows from the condition $\beta\left(\phi_{f}\right)=1$ for the end of the slow roll era that

$$
2 \gamma \tan \left(\gamma \hat{\phi}_{f}\right)=1 \quad \text { or } \quad \sin ^{2}\left(\gamma \hat{\phi}_{f}\right)=\frac{1}{1+4 \gamma^{2}}
$$

Inserting Equations (6.31.67) and (6.31.68) into Equation (6.31.64) gives

$$
\hat{\beta}(N)=\frac{2 \gamma}{\sqrt{\left(1+4 \gamma^{2}\right) e^{4 \gamma^{2} N}-1}} .
$$

Inserting this into Equation (5.134) we get for $\delta_{n s}$ and $r$

$$
\delta_{n s}=4 \gamma^{2} \frac{\left(1+4 \gamma^{2}\right) e^{4 \gamma^{2} N}+1}{\left(1+4 \gamma^{2}\right) e^{4 \gamma^{2} N}-1} \quad, \quad r=\frac{32 \gamma^{2}}{\left(1+4 \gamma^{2}\right) e^{4 \gamma^{2} N}-1} .
$$

It follows from these expressions that

$$
N=\frac{1}{\delta_{n s}-r / 4} \ln \frac{8 \delta_{n s}-r}{\left(1+\delta_{n s}-r / 4\right) r} \approx \frac{1}{\delta_{n s}-r / 4} \ln \left(8 \frac{\delta_{n s}}{r}-1\right) .
$$

The BPK-data, $\delta_{n s}=0.032, r<0.04$ require $N>77$.

\subsection{Warm Inflation}

In the usual (cold) inflationary models dissipative effects with decay of inflaton energy into radiation energy are neglected. However, during the evolution of warm inflation dissipative effects are important, and inflaton field energy is transformed to radiation energy. This was first taken into account in the construction of inflationary universe models by A. Berera [179] who introduced a new class of inflationary universe models called Warm Inflation. Further references to works prior to 2009 on warm inflation are found in $[180,181]$. For later works see [182-184] and references in these articles. 
The most recent articles are $[185,186]$. In this scenario there is no need for a reheating at the end of the inflationary era. The universe heats up and becomes radiation dominated during the inflationary era, so there is a smooth transition to a radiation dominated phase.

\subsubsection{General Warm Inflation Equations}

During the warm inflation era both the inflaton field energy with density $\rho_{\phi}$ and the electromagnetic radiation with energy density $\rho_{\gamma}$ are important for the evolution of the universe. The Friedmann Equation (2.2) is generalized to

$$
H^{2}=\frac{\kappa}{3}\left(\rho_{\phi}+\rho_{\gamma}\right)
$$

In these models the continuity Equation (2.3) for the inflaton field and the radiation take the form

$$
\dot{\rho}_{\phi}+3 H\left(\rho_{\phi}+p_{\phi}\right)=-\Gamma \dot{\phi}^{2}, \dot{\rho}_{\gamma}+4 H \rho_{\gamma}=\Gamma \dot{\phi}^{2},
$$

respectively, where $\Gamma$ is a dissipation coefficient of a dissipative process which causes decay of dark energy into radiation. In general $\Gamma$ is temperature dependent. It follows from these equations that the evolution equation for the inflaton takes the form

$$
\ddot{\phi}+3 H \dot{\phi}+V^{\prime}=-\Gamma \dot{\phi}
$$

in the case of warm inflation.

During warm inflation the dark energy predominates over radiation, i.e., $\rho_{\phi}>>\rho_{\gamma}$. During inflation $H, \phi$ and $\Gamma$ are slowly varying and the production of radiation is quasi-static, $\ddot{\phi}<<H \dot{\phi}$, $\dot{\rho}_{\gamma}<<4 H \rho_{\gamma}$. Then Equations (2.2), (6.32.2) and (6.32.3) take the form

$$
3 H^{2}=\kappa \rho_{\phi}=\kappa V, \quad 4 H \rho_{\gamma}=\Gamma \dot{\phi}^{2}, \quad(3 H+\Gamma) \dot{\phi}=-V^{\prime} .
$$

Hence in these models all of the radiation energy during the inflationary era comes from dissipation of the inflaton energy. In orther words, without dissiopation there is no radiation energy in the slow roll approximation. The last equation may be written as

$$
3 H(1+Q) \dot{\phi}=-V^{\prime}, Q \equiv \Gamma / 3 H
$$

Here $Q$ is called the dissipative ratio. Using Equation (6.32.2) the energy density of the radiation during warm inflation is given by

$$
\rho_{\gamma}=C_{\gamma} T^{4}=(3 / 4) Q \dot{\phi}^{2}
$$

where $C_{\gamma}$ is a constant and $T$ is the temperature of the radiation. The last two equations together with the slow roll approximation $3 H^{2}=\kappa V$ lead to the following expression for the temperature of the radiation

$$
T=\left[\frac{Q}{4 C_{\gamma}(1+Q)^{2}} \frac{V^{\prime 2}}{V}\right]^{1 / 4} .
$$

It follows from this equation and the first of the equations in (6.32.4) that the ratio of the energy densities of the radiation and the inflaton field are

$$
\frac{\rho_{\gamma}}{\rho_{\phi}}=\frac{3 Q \dot{\phi}^{2}}{4 V}=\frac{\kappa Q \dot{\phi}^{2}}{4 H^{2}} .
$$


Differentiating the first of Equation (6.32.4) and using that $\dot{V}=V^{\prime} \dot{\phi}$ we get $2 H \dot{H}=(\kappa / 3) V^{\prime} \dot{\phi}$. Inserting the expression for $V^{\prime}$ from Equation (6.32.6) leads to

$$
\dot{\phi}^{2}=-\frac{2 \dot{H}}{\kappa(1+Q)} .
$$

Hence we get

$$
\frac{\rho_{\gamma}}{\rho_{\phi}}=-\frac{1}{2} \frac{Q}{1+Q} \frac{\dot{H}}{H^{2}} .
$$

The Hubble slow roll parameters are

$$
\varepsilon_{H} \equiv-\frac{\dot{H}}{H^{2}}, \quad \eta_{H} \equiv-\frac{\ddot{H}}{2 H \dot{H}},
$$

giving

$$
\frac{\rho_{\gamma}}{\rho_{\phi}}=\frac{1}{2} \frac{Q}{1+Q} \varepsilon_{H} .
$$

This shows that during the slow roll era the energy density of radiation energy is much less than the the energy density of the inflaton field.

In the warm inflation scenario a thermalized radiation component is present with temperature $T>H$, where both $T$ and $H$ are expressed in units of energy. Then the tensor-to-scalar ratio is modified with respect to standard cold inflation, so that [187]

$$
r_{W}=\frac{H / T}{(1+Q)^{5 / 2}} r
$$

Hence, the tensor-to-scalar ratio is suppressed by the factor $(T / H)(1+Q)^{5 / 2}$ compared with the standard cold inflation.

Hall, Moss and Berera [188] have calculated the spectral index in warm inflation for the strong dissipative regime with $Q>>1$ or $\Gamma>>3 H$. We shall here follow Visinelli [189] and permit arbitrary values of $Q$. Equation (6.32.9) can be written

$$
\dot{H}=-(\kappa / 2)(1+Q) \dot{\phi}^{2},
$$

which replaces the corresponding cold inflation Equation (2.12). Using Equations (3.1), (6.32.4), (6.32.6), (6.32.14) and finally (6.32.9) we get

$$
\varepsilon=\frac{1}{2 \kappa}\left(\frac{V^{\prime}}{V}\right)^{2}=\frac{\kappa}{2}\left[\frac{(1+Q) \dot{\phi}}{H}\right]^{2}=-(1+Q) \frac{\dot{H}}{H^{2}} .
$$

The Hubble slow roll parameters can be written

$$
\varepsilon_{H} \equiv \frac{1}{2(1+Q)}\left(\frac{V^{\prime}}{V}\right)^{2} \quad, \quad \eta_{H} \equiv \frac{1}{1+Q}\left[\frac{V^{\prime \prime}}{V}-\frac{1}{2}\left(\frac{V^{\prime}}{V}\right)^{2}\right] .
$$

Hence

$$
\varepsilon=(1+Q) \varepsilon_{H}
$$


During the inflationary era the energy density of the radiation is much less than the energy density of the inflaton field, $\rho_{\gamma}<<\rho_{\phi}$. Using Equation (6.32.12) this condition takes the form

$$
\varepsilon_{H}<<2 \frac{1+Q}{Q} .
$$

In the strong dissipation regime this reduces to $\varepsilon_{H}<<2$.

Hall, Moss and Berera [190] have defined the end of the slow roll era for warm inflation by the condition $\varepsilon_{H}=1$ which in the strong dissipation regime corresponds to $\rho_{\gamma}=\rho_{\phi} / 2$. Note that $\varepsilon_{H}=1$ means $\dot{H}+H^{2}=0$ and therefore $\ddot{a}=a\left(\dot{H}+H^{2}\right)=0$. Hence for these models inflation ends with a transition from accelerated expansion to linear expansion, like that of the empty Milne universe.

Differentiation of Equation (6.32.6) and using that $(\dot{\phi})^{\prime}=\ddot{\phi} / \dot{\phi}$ gives

$$
V^{\prime \prime}=\frac{\Gamma^{\prime} V^{\prime}}{\Gamma+3 H}-3 H(1+Q) \frac{\ddot{\phi}}{\dot{\phi}}-3 \dot{H} \text {. }
$$

Dividing by $\kappa V$ and using the first of Equation (6.32.4) in the two last terms lead to

$$
\eta=\frac{1}{\kappa} \frac{V^{\prime \prime}}{V}=\frac{Q}{1+Q} \frac{1}{\kappa} \frac{\Gamma^{\prime} V^{\prime}}{\Gamma V}-\frac{1+Q}{H} \frac{\ddot{\phi}}{\dot{\phi}}-\frac{\dot{H}}{H^{2}} .
$$

Using Equation (6.32.19) we get

$$
\frac{\ddot{\phi}}{H \dot{\phi}}=\frac{1}{1+Q}\left(\frac{\varepsilon+Q \beta}{1+Q}-\eta\right), \beta \equiv \frac{1}{\kappa} \frac{\Gamma^{\prime} V^{\prime}}{\Gamma V},
$$

in agreement with Equation (2.14) of Visinelli [189]. Hall, Moss and Berera [190] and Rezazadeh et al. [173] have calculated that in the strong dissipative regime the scalar spectral index parameter, the tensor spectral index and the tensor-to-scalar ratio are

$$
\delta_{n s}=\frac{3}{2 Q}\left[\frac{3}{2}(\varepsilon+\beta)-\eta\right] \quad, \quad n_{t}=-\frac{2 \varepsilon}{Q}, \quad r=\frac{1}{6 M_{P}}(2 \sigma V)^{1 / 4}\left(\frac{\varepsilon}{Q^{3}}\right)^{3 / 4}
$$

where $\sigma$ is the Stefan-Boltzmann constant.

It may be noted that in warm inflation the condition for slow roll is that the absolute values of $\varepsilon, \eta$ and $\beta$ are much smaller than $1+Q$.

Moss and Xiong [191] have deduced a general expression for the scalar spectral tilt in warm inflation, and Herrera [192] has shown that when the dissipation coefficient is independent of the temperature, their expression takes the form

$$
\delta_{n s}=\frac{17+6 Q}{4(1+Q)^{2}} \varepsilon+\frac{1+9 Q}{4(1+Q)^{2}} \beta-\frac{3}{2} \frac{1}{1+Q} \eta .
$$

In the weak dissipative regime this expression reduces to

$$
\delta_{n s}=\frac{1}{4}(17 \varepsilon+\beta-6 \eta)
$$

\subsubsection{Warm Polynomial Inflation}

Visinelli [193] has investigated warm inflation with a polynomial potential which we write in the form

$$
V=M^{4} \hat{\phi}^{p}
$$


where $\hat{\phi}=\phi / M_{P}$ since the potential and the inflaton field have dimension equal to the fourth and first power of energy, respectively. Here $M$ represents the energy scale of the potential when the inflaton field has Planck mass. Furthermore he assumes that the dissipative term is also monomial

$$
\Gamma=\Gamma_{0} \hat{\phi}^{q / 2}
$$

He considered models with $p>0$ and $q>p$. However in the present article we shall also consider polynomial models with $p<0$. Note that $q=0$ corresponds to a constant dissipation coefficient.

From Equations (6.32.25) and (6.32.26) we have

$$
Q=Q_{0} \hat{\phi}^{\frac{q-p}{2}} \quad, \quad Q_{0}=\frac{\Gamma_{0} M_{P}}{\sqrt{3} M^{2}}
$$

The constant $Q_{0}$ represents the strength of the dissipation. For $q=p$ the dissipative ratio is constant, $Q=Q_{0}$. We shall here consider the strong dissipative regime where $Q>>1$. Then the third of Equations (6.32.4) reduces to

$$
\dot{\phi}=-\frac{V^{\prime}}{\Gamma} \text {. }
$$

Inserting Equations (6.32.25) and (6.32.26) gives

$$
\dot{\phi}=-\frac{p M^{4}}{\Gamma_{0} M_{P}} \hat{\phi}^{p-\frac{q}{2}-1} .
$$

Integration leads to

$$
\phi(t)=\left[\frac{4+q-2 p}{2}\left(K-\frac{p M^{4}}{\Gamma_{0} M^{p-\frac{q}{2}}} t\right)\right]^{\frac{2}{4+q-2 p}} \quad, \quad q>2(p-2) .
$$

where $K$ is a constant of integration. The initial condition $\phi(0)=0$ gives $K=0$.

The special cases (i) $\Gamma=V / M_{P}^{3}$, i.e., $\Gamma_{0}=M^{4} / M_{P}^{3}, q=2 p$ and (ii) $\Gamma=\Gamma_{0}$, i.e., $q=0$, both with the initial condition $\phi(0)=0$, i.e, $K=0$, have been considered by Sharif and Saleem [194]. The latter case was originally considered by Taylor and Berera [195]. For these cases the condition $\phi(t)>0$ requires $p<0$. In the first case Equation (6.32.32) reduces to

$$
\hat{\phi}=\sqrt{-2 p M_{P} t}
$$

Note that the time has dimension inverse mass with the present units, so that $M_{P} t$ is dimensionless.

Visinelli, however, has considered polynomial models with $p>0$. Then we have to change the initial condition. The corresponding solution of Equation (6.32.31) with $q=2 p$ and the inflaton field equal to the Planck mass at the Planck time gives

$$
\hat{\phi}=\sqrt{1-2 p M_{P}\left(t-t_{P}\right)} .
$$

It may be noted that $q=2(p-2)$ gives a different time evolution of the inflaton field. Then Equation (6.32.29) with the boundary condition $\phi\left(t_{P}\right)=M_{P}$ has the solution

$$
\hat{\phi}=\exp \left[-\frac{p M^{4}}{\Gamma_{0} M_{P}^{2}}\left(t-t_{P}\right)\right]
$$

In this case the inflaton field decreases or increases exponentially, depending upon the sign of $p$. 
Inserting Equations (6.32.25) and (6.32.27) into Equations (6.32.15), (6.32.20) and (6.32.21), the slow-roll parameters are

$$
\varepsilon=\frac{p^{2}}{2} \frac{1}{\hat{\phi}^{2}} \quad, \quad \eta=\frac{2(p-1)}{p} \varepsilon, \quad \beta=\frac{q}{p} \varepsilon .
$$

With these expressions Equation (6.32.23) valid in the regime of strong dissipation, $Q>>1$, gives

$$
\delta_{n s}=\frac{3(4+3 q-p)}{4 p} \frac{\varepsilon}{Q}
$$

The slow-roll regime ends when at least one of the parameters (6.32.34) is not much smaller than $1+Q$. In the strong dissipative regime $Q>>1$ and $\varepsilon_{f}=Q_{f}$. Using Equations (6.32.27) and (6.32.34) we then get

$$
\hat{\phi}_{f}=\left(\frac{p^{2}}{2 Q_{0}}\right)^{\frac{2}{4+q-p}} .
$$

The number of e-folds, $N$, in the slow roll era for this model has been calculated by Visinelli [193]. It is defined by

$$
N=\ln \frac{a_{f}}{a}=\int_{t}^{t_{f}} H d t=\int_{\phi}^{\phi_{f}} \frac{H}{\dot{\phi}} d \phi .
$$

Using Equations (6.32.4) and (6.32.6) we get

$$
N=\frac{1}{M_{P}^{2}} \int_{\phi_{f}}^{\phi}(1+Q) \frac{V}{V^{\prime}} d \phi .
$$

Inserting the potential (6.32.25), performing the integration and considering the strong dissipative regime, gives

$$
N \approx \frac{2 Q_{0}}{p(4+q-p)}\left(\hat{\phi}^{\frac{4+q-p}{2}}-\hat{\phi}_{f}^{\frac{4+q-p}{2}}\right) .
$$

The time dependence of the inflaton field is given by Equation (6.32.32) when $p<0$ showing that $\phi_{f}>\phi$ in this case, and by Equation (6.32.32) when $p>0$ implying $\phi_{f}<\phi$ in that case, showing that $N>0$ in both cases.

$$
\hat{\phi} \approx\left(\frac{p(4+q-p) N}{2 Q_{0}}\right)^{\frac{2}{4+q-p}} .
$$

Inserting this into the first of Equation (6.32.34) and Equation (6.32.27) gives

$$
\varepsilon \approx \frac{p^{2}}{2}\left[\frac{2 Q_{0}}{p(4+q-p) N}\right]^{\frac{4}{4+q-p}} \quad, \quad Q \approx Q_{0}\left[\frac{p(4+q-p) N}{2 Q_{0}}\right]^{\frac{q-p}{4+q-p}}
$$

Inserting these expressions into Equation (6.32.35) gives

$$
\delta_{n s} \approx \frac{3(4+3 q-p)}{4(4+q-p)} \frac{1}{N}
$$

Note that with $q=0$, i.e., a constant value of the dissipation parameter $\Gamma$, this equation reduces to

$$
\delta_{n s}=\frac{3}{4 N}
$$


for all values of $p$. Then $N=60$ gives $\delta_{n s}=0.012$ which is smaller than the preferred value from the Planck data, $\delta_{n s}=0.032$. Inserting $q=2 p$ in Equation (6.32.44) and solving the equation with respect to $p$ gives,

$$
p=\frac{4\left(4 N \delta_{n s}-3\right)}{15-4 N \delta_{n s}} .
$$

The Planck values $\delta_{n s}=0.032, N=60$ give $p=2.56$ and $q=5.11$, and hence is negative in conflict with the Planck observations.

Panotopoulos and Videla [183] have investigated the tensor-to-scalar ratio in this model with $p=4$. According to Equation (6.1.36) $\delta_{n s}=2 / N$ in this case for cold inflation. For $\delta_{n s}=0.032$ this corresponds to $N \approx 62$ which is an acceptable number of e-folds. Then Table 1 shows that the tensor-to-scalar ratio is $r=0.32$, which is much larger than allowed by the Planck observations. Furthermore, in this case case $r=8 \delta_{n s}$. Panotopoulos and Videla found the corresponding $\delta_{n s}$, $r$ - relation in warm inflation with $\Gamma=a T$, where $\mathrm{a}$ is a dimensionless parameter. They considered two cases.

A. The weak dissipative regime. In this case $Q<<1$ and Equation (6.32.13) reduces to $r_{W}=(H / T) r$. They then found

$$
r_{W} \approx \frac{0.01}{\sqrt{a}} \delta_{n s}
$$

With $\delta_{n s}=0.032$ and $r_{W}<0.12$ this requires $a>7 \times 10^{-6}$. However, they also found that in this case $\delta_{n s}=1 / N$ giving $N=31$ which is too small to be compatible with the standard inflationary scenario.

B. The strong dissipative regime. Then $Q>>1$ and $r_{W} \approx\left(H / T Q^{5 / 2}\right) r$. They then found

$$
\delta_{n s}=\frac{45}{28 N} \quad, \quad r_{W}=\frac{3.8 \times 10^{-7}}{a^{4}} \delta_{n s} .
$$

Then $N=50$ and $a>1.8 \times 10^{-2}$, so this is a promising model.

Taylor and Berera [195] have briefly considered warm inflation models with an exponential potential, $V=V_{0} \exp (\phi / M)$, and found that in the strong dissipation regime the scalar spectral index parameter $\delta_{n s}$ for models of this type is

$$
\delta_{n s}=-\frac{3}{8}\left(\frac{M_{P}}{M}\right)^{2} \frac{1}{Q^{\prime}}
$$

and hence is negative in conflict with the Planck observations.

\subsubsection{Warm Natural Inflation}

Visinelli [188] has investigated warm natural inflation with potential

$$
V(\phi)=V_{0}(1+\cos \widetilde{\phi})=2 V_{0} \cos ^{2}(\widetilde{\phi} / 2) .
$$

Here $\widetilde{\phi}=\phi / M$, and $M$ is the spontaneous symmetry breaking scale. He assumed that the dissipation coefficient $\Gamma$ is independent of the inflaton field, so that $\beta=0$. Inserting the definitions of $\varepsilon$ and $\eta$ into Equation (6.32.23) then gives

$$
\delta_{n s}=\frac{3}{2 \kappa Q V^{2}}\left(\frac{3}{4} V^{\prime 2}-V V^{\prime \prime}\right) .
$$

Inserting the potential gives

$$
\delta_{n s}=\frac{3}{8 \kappa Q} \frac{3-c_{\phi}}{1+c_{\phi}},
$$


where $c_{\phi}$ is given in Equation (6.5.3). From the first of the Equation (6.32.4) with the potential (6.32.48) we have

$$
H=\sqrt{(\kappa / 3) V_{0}\left(1+c_{\phi}\right)} .
$$

Equations (6.32.5) and (6.32.51) then give

$$
Q=\frac{\Gamma}{\sqrt{3 \kappa V_{0}\left(1+c_{\phi}\right)}} .
$$

Hence

$$
\delta_{n s}=A \frac{3-c_{\phi}}{\sqrt{1+c_{\phi}}}, \quad A=\frac{3}{8 \Gamma} \sqrt{\frac{3 V_{0}}{\kappa}} .
$$

We shall now express the $\delta_{n s}$ in terms of the number of e-folds of expansion during the slow roll era for this inflationary universe model, again following Visinelli. Assuming that the dissipation parameter $\Gamma$ is independent of $\phi$, i.e., that $\beta=0$, the number of e-folds is given by

$$
N \simeq-\Gamma \int_{\phi_{i}}^{\phi_{f}} \frac{H(\phi)}{V^{\prime}(\phi)} d \phi
$$

Differentiating the potential (6.32.48) and inserting Equation (6.32.51) we get

$$
N=\frac{\alpha}{2} \int_{\widetilde{\phi}_{i}}^{\widetilde{\phi}_{f}} \frac{\sqrt{1+\cos x}}{\sin x} d x=\frac{\alpha}{\sqrt{2}} \ln \frac{\tan \left(\widetilde{\phi}_{f} / 4\right)}{\tan \left(\widetilde{\phi}_{i} / 4\right)} .
$$

Hence,

$$
\tan \frac{\widetilde{\phi}_{i}}{4}=\tan \frac{\widetilde{\phi}_{f}}{4} \exp \left(-\frac{\beta N}{2}\right)
$$

Visinelli has argued that

$$
\widetilde{\phi}_{f}=\pi-\beta,
$$

giving

$$
\tan \frac{\widetilde{\phi}_{f}}{4}=\frac{1-\tan (\beta / 4)}{1+\tan (\beta / 4)} .
$$

Inserting this into Equation (6.32.56) gives

$$
\tan \frac{\widetilde{\phi}_{i}}{4}=\gamma \exp \left(-\frac{\beta N}{2}\right), \quad \gamma=\frac{1-\tan (\beta / 4)}{1+\tan (\beta / 4)}
$$

Applying the trigonometric identity

$$
\sqrt{1+\cos \theta}=\sqrt{2} \frac{1-\tan ^{2}(\theta / 4)}{1+\tan ^{2}(\theta / 4)},
$$

in the expression (6.32.59) and inserting the result into Equation (6.32.53) we finally arrive at

$$
\delta_{n s}=\frac{3}{8} \beta \frac{\exp (2 \beta N)+\gamma^{4}}{\exp (2 \beta N)-\gamma^{4}} .
$$


Here we must have $\beta<<1$ in order to give the Planck value $\delta_{n s}=0.032$ for $N=60$. Hence, Equation (6.32.59) gives $\gamma \approx 1$. A good approximation for $\delta_{n s}$ is therefore

$$
\delta_{n s} \approx(3 / 8) \beta \operatorname{coth}(\beta N) .
$$

Inserting $\delta_{n s}=0.032$ and $N=60$ gives $\beta=0.08$.

Visinelli [188] found that the tensor-to-scalar ratio for this inflationary model is

$$
r=128 \kappa \sqrt{\frac{\pi}{\Gamma}} \frac{\dot{\phi}^{2}}{T \sqrt{H}}
$$

Differentiating the expression (6.32.51) gives

$$
\dot{H}=-\frac{\kappa V_{0}}{6 M} \frac{s_{\phi} \dot{\phi}}{H}, s_{\phi} \equiv \sin \widetilde{\phi}
$$

Combining this with Equation (6.32.14) in the strong dissipative regime and using Equation (6.32.5) gives

$$
\dot{\phi}=\frac{V_{0} s_{\phi}}{M \Gamma} .
$$

The energy density of the radiation is

$$
\rho_{\gamma}=a T^{4}
$$

where $a=7 \times 5657 \times 10^{-16} \mathrm{~J} \cdot \mathrm{m}^{-3} \cdot \mathrm{K}^{-4}=4.69 \times 10^{-6} \mathrm{GeV} \cdot \mathrm{m}^{-3} \cdot \mathrm{K}^{-4}$ is the radiation constant. Combining with Equation (6.32.7) we get

$$
T=\left(\frac{\Gamma}{4 a H}\right)^{1 / 4} \dot{\phi}^{1 / 2}
$$

Equations (6.32.63), (6.32.65) and (6.32.66) give

$$
r=B \frac{s_{\phi}^{3 / 2}}{\left(1+c_{\phi}\right)^{1 / 8}}, \quad B=\frac{384 \cdot 3^{5 / 8} \kappa^{7 / 8} \sqrt{6 \pi} V^{11 / 8} a^{1 / 4}}{M^{3 / 2} \Gamma^{9 / 4}} .
$$

Visinelli [188] has evaluated the constant $B$ and concluded that for this type of inflationary universe model the expected value of $r$ is extremely small. If observations give a value $r>10^{-14}$ this model has to be abandoned.

\subsubsection{Warm Viscous Inflation}

As noted by del Campo, Herrera and Pavón [196], it has been usual, for the sake of simplicity, to study warm inflation models containing an inflaton field and radiation, only, ignoring the existence of particles with mass that will appear due to the decay of the inflaton field. However, these particles modify the fluid pressure in two ways: (i) The relation between pressure and energy density is no longer $p=(1 / 3) \rho$ as it is for radiation. A simple generalization is to describe the mixture of matter and radiation as a fluid with equation of state $p=(\gamma-1) \rho$, where $\gamma$ is the adiabatic constant with value $0 \leq \gamma \leq 2$. (ii) Due to interactions between the particles and the radiation there will appear a bulk viscosity so that the effective pressure takes the form $p_{e f f}=p+p_{\zeta}$, where

$$
p_{\zeta}=-3 \zeta H,
$$

is a "viscous pressure" and $\varsigma$ a coefficient of bulk viscosity. 
Following Setare and Kamali [197] the second of the Equation (6.32.2) is now generalized to

$$
\dot{\rho}+3 H(\rho+p-3 \zeta H)=\Gamma \dot{\phi}^{2} .
$$

With quasi static production of radiation we have $\dot{\rho}<<3 H(\gamma \rho-3 \zeta H)$ and $\dot{\rho}<<\Gamma \dot{\phi}^{2}$. The Equation (6.32.70) reduces to

$$
\gamma \rho=Q \dot{\phi}^{2}+3 \zeta H
$$

Equations (3.7) and (6.32.9) give

$$
\varepsilon_{H}=\frac{\kappa}{2}(1+Q) \frac{\dot{\phi}^{2}}{H^{2}} .
$$

Using Equation (6.32.1) with $\rho_{\gamma}=0$ we get

$$
(2 / 3) \varepsilon_{H} \rho_{\phi}=(1+Q) \dot{\phi}^{2}
$$

Let us for a moment consider the strong dissipative regime with $Q>>1$. Then Equation (6.32.73) reduces to

$$
(2 / 3) \varepsilon_{H} \rho_{\phi}=Q \dot{\phi}^{2}
$$

In this case we get

$$
\gamma \rho=(2 / 3) \varepsilon_{H} \rho_{\phi}+3 \zeta H .
$$

Hence the slow roll condition $\varepsilon_{H}<<1$ requires that

$$
\rho_{\phi}>>(3 / 2)(\gamma \rho-3 \zeta H) .
$$

\subsubsection{Warm Viscous Intermediate Inflation}

We shall now consider the warm, viscous, intermediate inflation models studied by Setare and Kamali [197] in the strong dissipation regime. This is also similar to the isotropic universe models corresponding to the anisotropic models considered by Sharif and Saleem [194]. The scale factor is given in Equation (6.30.1) and the Hubble parameters and its derivatives are given in Equation (6.30.2), where the expressions must be multiplied by $A$ in the present case. In order to find the time evolution of the inflaton field we now write Equation (6.32.9) as

$$
\dot{\hat{\phi}}= \pm \sqrt{-2 \dot{H} /(1+Q)} .
$$

Inserting the expression (6.30.2) for $\dot{H}$ into Equation (6.32.77) gives

$$
\dot{\hat{\phi}}= \pm \sqrt{\frac{2 \alpha(1-\alpha)}{1+Q}} t_{P}^{-1} \hat{t}^{\frac{\alpha}{2}-1} .
$$

where $t_{P}$ is the Planck time. Note that this requires $0<\alpha<1$.

Both Setare and Kamali [197] and Sharif and Saleem [194] have considered two cases. In the first one assumes

$$
\Gamma=\Gamma(\phi)=V(\phi), \zeta=\zeta_{1} \rho .
$$

Equations (6.32.4) and (6.32.5) then give

$$
Q=t_{P} H
$$


Furthermore, for several reasons, the authors restricted their analysis to the strong dissipative regime where $Q>>1$. Equations (6.32.77) and (6.32.78) then reduce to

$$
\dot{\hat{\phi}}= \pm \sqrt{-\frac{2 \dot{H}}{t_{P} H}}=\sqrt{2(1-\alpha)}\left(t_{P} t\right)^{-1 / 2},
$$

where we have used Equation (6.30.2). Integrating with the initial condition $\phi(0)=0$ and assuming that $\phi(t)>0$ we get

$$
\hat{\phi}(t)=2 \sqrt{2(1-\alpha) \hat{t}}
$$

which is different from the corresponding Equation (6.31.5) for cold intermediate inflation. We see that $\phi$ is an increasing function of time. Inserting the expression (6.30.2) for $H$ into the first of the Equation (6.32.4) gives

$$
V(t)=3 \alpha^{2} M_{P}^{4} \hat{t}^{2(\alpha-1)} .
$$

Hence the potential decreases with time. Combining this with Equation (6.32.82) leads to

$$
V(\phi)=3 \alpha^{2} M_{P}^{4}\left(\frac{\hat{\phi}}{2 \sqrt{2(1-\alpha)}}\right)^{4(\alpha-1)} .
$$

Inserting the expressions (6.32.79) into Equation (6.32.71) we obtain

$$
\rho=\frac{V \dot{\phi}^{2}}{3 H\left(\gamma-3 \zeta_{1} H\right)} .
$$

Hence in order that the density of the mixture of matter and radiation shall be positive, we must have $\zeta_{1}<\gamma / 3 H$. Inserting the expressions (6.30.2), (6.32.81) and (6.32.83) we find the evolution of the density with time,

$$
\rho=\frac{2 \alpha(1-\alpha) M_{P}^{4} \hat{t}^{\alpha-2}}{\gamma-3 \zeta_{1} \alpha M_{P} \hat{t}^{\alpha-1}} .
$$

Solving Equation (6.32.81) with respect to time gives

$$
\hat{t}=\frac{\hat{\phi}^{2}}{8(1-\alpha)} .
$$

Inserting this into Equation (6.32.86) gives the density as a function of the inflaton field,

$$
\rho=\frac{2 \alpha(1-\alpha) M_{P}^{4}[\hat{\phi} / 2 \sqrt{2(1-\alpha)}]^{2(\alpha-2)}}{\gamma-3 \zeta_{1} \alpha M_{P}[\hat{\phi} / 2 \sqrt{2(1-\alpha)}]^{2(\alpha-1)}} .
$$

Setare and Kamali [197] and Sharif and Saleem [194] used the Hubble slow roll parameters (3.7). In the strong dissipative regime with $Q>>1$ they take the form

$$
\varepsilon_{H}=-\frac{\dot{H}}{H^{2}}=\frac{1}{2 Q}\left(\frac{V^{\prime}}{V}\right)^{2}, \quad \eta_{H}=-\frac{\ddot{H}}{2 H \dot{H}}=\frac{1}{Q}\left[\frac{V^{\prime \prime}}{V}-\frac{1}{2}\left(\frac{V^{\prime}}{V}\right)^{2}\right] .
$$

Furthermore Setare and Kamali have shown that for this type of inflationary models the scalar spectral index parameter is given as

$$
\delta_{n s}=2 \eta_{H}-5 \varepsilon_{H}
$$


Differentiating the expression (6.32.83) gives

$$
\varepsilon_{H}=\frac{1-\alpha}{\alpha}\left(\frac{\hat{\phi}}{2 \sqrt{2(1-\alpha)}}\right)^{-2 \alpha} \quad, \quad \eta_{H}=\frac{3-2 \alpha}{2 \alpha}\left(\frac{\hat{\phi}}{2 \sqrt{2(1-\alpha)}}\right)^{-2 \alpha}=\frac{3-2 \alpha}{2(1-\alpha)} \varepsilon_{H} .
$$

Inserting the last expression for $\eta_{H}$ into Equation (6.32.91) gives

$$
\delta_{n s}=\frac{3 \alpha-2}{1-\alpha} \varepsilon_{H} .
$$

Together with the expression (6.32.91) for $\varepsilon_{H}$ this leads to

$$
\delta_{n s}=\frac{3 \alpha-2}{\alpha}\left(\frac{\hat{\phi}}{2 \sqrt{2(1-\alpha)}}\right)^{-2 \alpha} .
$$

Note that the scale independent Harrison-Zeldovich spectrum occurs for $\alpha=2 / 3$.

The slow roll era ends when the inflaton field has a value $\phi_{f}$ so that $\varepsilon_{H}\left(\phi_{f}\right)=1$, corresponding to $\varepsilon\left(\phi_{f}\right)=Q$, which gives

$$
\left(\frac{\hat{\phi}_{f}}{2 \sqrt{2(1-\alpha)}}\right)^{2 \alpha}=\frac{1-\alpha}{\alpha} .
$$

The number of e-folds is given by Equation (6.32.40), which in the present case takes the form

$$
N=\frac{1}{\sqrt{3} M_{P}} \int_{\phi}^{\phi_{f}} \frac{V^{3 / 2}}{V^{\prime}} d \phi
$$

Inserting the potential (6.32.84) and integrating gives

$$
N=\left(\frac{\hat{\phi}}{2 \sqrt{2(1-\alpha)}}\right)^{2 \alpha}-\left(\frac{\hat{\phi}_{f}}{2 \sqrt{2(1-\alpha)}}\right)^{2 \alpha}=\left(\frac{\hat{\phi}}{2 \sqrt{2(1-\alpha)}}\right)^{2 \alpha}-\frac{1-\alpha}{\alpha} .
$$

Hence

$$
\left(\frac{\hat{\phi}}{2 \sqrt{2(1-\alpha)}}\right)^{2 \alpha}=\frac{N \alpha+1-\alpha}{\alpha} .
$$

Inserting Equation (6.32.97) into Equation (6.32.93) leads to

$$
\delta_{n s}=\frac{3 \alpha-2}{N \alpha+1-\alpha} .
$$

A positive value of $\delta_{n s}$ requires $\alpha>2 / 3$. Equation (6.32.98) can be written

$$
\alpha=\frac{2+\delta_{n s}}{3+\delta_{n s}-N \delta_{n s}} \approx \frac{2}{3-N \delta_{n s}} .
$$

Inserting the Planck value $\delta_{n s}=0.032$ and $N=60$ gives $\alpha \approx 1.9$. This is in conflict with the requirement $0<\alpha<1$. The values $2 / 3<\alpha<1$ give $0<\delta_{n s}<1 / N=0.16$ which is smaller than allowed by the BPK-data. 
In the second case Setare and Kamali and Sharif and Saleem assumed that $\Gamma=\Gamma_{0}, \zeta=\zeta_{0}$. Equation (6.4) then takes the form. Using Equations (6.32.79) and (6.30.2) and integrating with the initial condition $\phi(0)=0$, leads to

$$
\phi(t)=\phi_{1} \hat{t}^{\alpha-1 / 2} \quad, \quad H(\phi)=\alpha M_{P} \widetilde{\phi}^{\frac{2(\alpha-1)}{2 \alpha-1}} \quad, \quad \phi_{1}=\frac{2 \alpha M_{P}}{2 \alpha-1} \sqrt{\frac{6 M_{P}(1-\alpha)}{\Gamma_{0}}} .
$$

where $\widetilde{\phi}=\phi / \phi_{1}$. Hence from the first of the equations Equation (4.32.4) we have

$$
V(\phi)=3 \alpha^{2} M_{P}^{4} \widetilde{\phi}^{\frac{4(1-\alpha)}{2 \alpha-1}} .
$$

From Equations (6.32.1) and (6.32.100) the viscous pressure is

$$
p_{\zeta}=-3 \zeta_{0} \alpha M_{P}^{2} \widetilde{\phi}^{\frac{2(1-\alpha)}{2 \alpha-1}}
$$

In the present case Equation (6.32.84) is replaced by

$$
\gamma \rho=\frac{\Gamma_{0}}{3} \frac{\dot{\phi}^{2}}{H}+3 \zeta_{0} H
$$

Differentiation the first of Equation (4.32.100) we have

$$
\dot{\phi}=\frac{\phi_{1} M_{P}}{2}(2 \alpha-1) \hat{t}^{\alpha-\frac{3}{2}} .
$$

Inserting this, together with the expression (6.31.2) for $H$ into Equation (6.32.103) gives the time evolution of the density of the matter-radiation fluid,

$$
\rho=\frac{M_{P}}{3 \gamma \alpha}\left[\Gamma_{0} \phi_{0}^{2}\left(\alpha-\frac{1}{2}\right)^{2} \hat{t}^{\alpha-2}+\zeta_{0}(3 \alpha)^{2} \hat{t}^{\alpha-1}\right] .
$$

Substituting for $t$ from Equation (6.32.99) we obtain the density as a function of the inflaton field

$$
\rho=\frac{M_{P}}{3 \gamma \alpha}\left[\Gamma_{0} \phi_{0}^{2}\left(\alpha-\frac{1}{2}\right)^{2} \widetilde{\phi}^{\frac{2(\alpha-2)}{2 \alpha-1}}+\zeta_{0}(3 \alpha)^{2} \widetilde{\phi}^{\frac{2(\alpha-1)}{2 \alpha-1}}\right] .
$$

In this case $\varepsilon_{H}$ and $\eta_{H}$ becomes

$$
\varepsilon_{H}=\frac{1-\alpha}{\alpha} \widetilde{\phi}^{-\frac{2 \alpha}{2 \alpha-1}} \quad, \quad \eta_{H}=\frac{2-\alpha}{2 \alpha} \widetilde{\phi}^{-\frac{2 \alpha}{2 \alpha-1}}=\frac{2-\alpha}{2(1-\alpha)} \varepsilon_{H} .
$$

The expression for $\eta_{H}$ is different for that of Setare and Kamali and will lead to a different conclusion than their concerning the acceptability of this class of inflation models. Inserting the expressions (6.32.107) into Equation (6.32.89), the scalar spectral index is

$$
\delta_{n s}=\frac{4 \alpha-3}{1-\alpha} \varepsilon_{H} .
$$

Hence,

$$
\delta_{n s}=\frac{4 \alpha-3}{\alpha} \widetilde{\phi}^{-\frac{2 \alpha}{2 \alpha-1}} .
$$

The final value of $\phi_{f}$ is given by

$$
\widetilde{\phi}^{\frac{2 \alpha}{2 \alpha-1}}=\frac{1-\alpha}{\alpha}
$$


The number of e-folds is

$$
N=\widetilde{\phi}^{\frac{2 \alpha}{2 \alpha-1}}-\widetilde{\phi}_{f}^{\frac{2 \alpha}{2 \alpha-1}}=\widetilde{\phi}^{\frac{2 \alpha}{2 \alpha-1}}-\frac{1-\alpha}{\alpha} .
$$

Hence

$$
\widetilde{\phi}^{\frac{2 \alpha}{2 \alpha-1}}=N+\frac{1-\alpha}{\alpha} .
$$

The scalar spectral index is

$$
\delta_{n s}=\frac{4 \alpha-3}{N \alpha+1-\alpha} \approx \frac{4 \alpha-3}{\alpha} \frac{1}{N^{\prime}}
$$

which can be written

$$
\alpha \approx \frac{3}{4-N \delta_{n s}} .
$$

Inserting the Planck value $\delta_{n s}=0.032$ and $N=60$ gives $\alpha=1.4$. This is outside the range allowed by Equation (4.32.78), $\alpha<1$, which requires $N<31$. Hence these models are not acceptable for realistic inflation. However in the anisotropic case considered by Sharif and Saleem, one may obtain agreement with the Planck data for $\alpha<1$. The tensor to scalar ratio has a very small value in these models.

In the case (i) considered above the dissipation coefficient is assumed to be proportional to the potential of the inflaton field, $\Gamma=\Gamma(\phi)=\kappa V(\phi) / M_{P}$. Combining this with the first of Equations (6.32.4) we get $\Gamma=3 H^{2} / M_{P}$. Furthermore in the strong dissipative regime with $\Gamma>>3 H$ this leads to $H>>M_{P}$. With $H \approx\left(1 / M_{P}\right) \sqrt{V / 3}$ these models have $V>>3 M_{P}^{4}$.

The slow roll era begins at a point of time $t_{i}$ when the inflaton field is given by Equation (6.32.81). Combining this with Equation (6.32.73) leads to

$$
t_{i}=\left(N+\frac{1-\alpha}{\alpha}\right)^{1 / \alpha} t_{P} \approx N^{1 / \alpha} t_{P}
$$

With $N=60, \alpha=1.4$ we get $t_{i} \approx 18 t_{P}$. A smaller value of $\alpha$ corresponds to a later beginning of the inflationary era.

V. Karmali and Setare have considered warm viscous inflation models in the context of brane cosmology using the so-called chaotic potential (4.1.1) with $\alpha=3 / 2$. The corresponding models in ordinary (not brane) spacetime correspond to taking the limit that the brane tension $\lambda \rightarrow \infty$ in their equations. They first considered the case $\Gamma=\Gamma_{0}$. Then the time evolution of the inflaton field is given by Equation (6.31.5) with $\alpha=3 / 2$. In this case $\delta_{n s}=0.012$ which is smaller than the preferred value from the Planck data. Karmali and Setare got a different result. Letting $\lambda \rightarrow \infty$ in their Equation (68) gives $\delta_{n s}=0$, i.e., a scale invariant spectrum.

Next they considered the case $\Gamma=\Gamma(\phi)=\beta V(\phi) / M_{P}^{3}$ where $\beta$ is a dimensionless constant. With $\beta=1$ this corresponds to the first case considered by Sharif and Saleem. It will now be shown that this model is in conflict with observations. With $V=3 M_{P}^{2} H^{2}$ we then have $\Gamma(\phi)=3 \beta H^{2} / M_{P}$. In the strong dissipation regime: $\Gamma>>H$. Hence $3 \beta H^{2}>>M_{P} H$, giving $3 \beta H>>M_{P}$.

We now use the constraints from the Planck satellite data, $H<1.8 \times 10^{13} \mathrm{GeV}$. This gives a lower bound on $\beta$ as $\beta>>M_{P} / 3 H=4.4 \times 10^{4}$. Hence the model with $\beta=1$ is in conflict with the Planck data. 
6.32.6. The N-Formalism Applied to Warm Inflation

We shall here follow the paper of R. Herrera [192] and use units so that $\kappa=1$. In warm inflation the relationships of the derivatives of $V$ with respect to $\phi$ and $N$, the first one corresponding to Equation (5.42) in cold inflation, are

$$
V^{\prime 2}=(1+Q) V V, N \quad V^{\prime \prime}=\frac{(1+Q)\left(V_{, N}^{2}+V V,_{N N}\right)+V V_{,_{N}} Q_{, N}}{2 V,,_{N}}
$$

Furthermore for the derivatives of the dissipation coefficient $\Gamma$ with respect to $\phi$ and $N$ we have

$$
\Gamma_{, \phi}=\left[\frac{(1+Q) V}{V, N}\right]^{1 / 2} \Gamma_{, N}
$$

Hence the potential slow roll parameters of warm inflation as given in Equations (6.32.15), (6.32.20) and (6.32.21) take the forms

$$
\varepsilon=(1+Q) \frac{V, N}{2 V} \quad, \quad \eta=\frac{(1+Q)\left(V_{,_{N}}^{2}+V V_{,_{N N}}\right)+V V_{,_{N}} Q_{,_{N}}}{2 V V, N} \quad, \quad \beta=(1+Q) \frac{\Gamma_{, N}}{\Gamma}
$$

The Equation (6.32.23) for the scalar spectral tilt can then be written

$$
\delta_{n s}=\frac{11+3 Q}{8(1+Q)} \frac{V_{, N}}{V}-\frac{3}{4} \frac{V_{, N N}}{V, N}+\frac{1+9 Q}{4(1+Q)} \frac{\Gamma_{, N}}{\Gamma}-\frac{3}{4} \frac{Q_{, N}}{1+Q}
$$

or using that $\Gamma=3 H Q$,

$$
\delta_{n s}=\frac{11+3 Q}{8(1+Q)} \frac{V_{,_{N}}}{V}-\frac{3}{4} \frac{V_{,_{N N}}}{V_{,_{N}}}+\frac{1+9 Q}{4(1+Q)} \frac{H_{,_{N}}}{H}+\frac{1}{4} \frac{1+6 Q}{1+Q} \frac{Q_{, N}}{Q} .
$$

Since $3 H^{2} \approx V$ in the slow roll approximation we obtain

$$
\delta_{n s}=\frac{1}{4}\left(6 \frac{V,_{N}}{V}-3 \frac{V,_{N N}}{V,_{N}}+\frac{1+6 Q}{1+Q} \frac{Q_{N}}{Q}\right) .
$$

Using Equations (6.32.4) and (6.32.118) it follows that Equation (6.32.13) for the tensor-to-scalar ratio for warm inflation takes the form

$$
r_{w}=\frac{8}{\sqrt{3}(1+Q)^{3 / 2}} \frac{V,_{N}}{T V^{3 / 2}} .
$$

I follows from Equations (3.62), (6.32.4), (6.32.5) and (6.32.116) that

$$
\phi=\phi_{0}+\int_{0}^{N}\left[\frac{V, N}{(1+Q) V}\right]^{1 / 2} d N
$$

From Equations (6.32.7) and (6.32.116) we have

$$
T=\left[\frac{Q}{4 C_{\gamma}(1+Q)} \frac{V, N}{V}\right]^{1 / 4}
$$

Hence the tensor-to-scalar ratio is

$$
r_{w}=8\left[\frac{4 C_{\gamma} V_{, N}^{3}}{9 Q(1+Q)^{5} V^{5}}\right]^{1 / 4}
$$


In the weak dissipative regime where $Q<<1$, the $\phi, N$-relation can be written

$$
\phi=\phi_{0}+\int_{0}^{N}\left(\frac{V, N}{V}\right)^{1 / 2} d N .
$$

Using Equations (5.32.124), (6.32.121) and (6.32.122) then reduce to

$$
\delta_{n s}=\frac{1}{4}\left(\ln \frac{Q V^{6}}{V_{N_{N}}^{3}}\right), N \quad, \quad r_{w}=8\left(\frac{4 C_{\gamma}}{9} \frac{V_{N_{N}}^{3}}{Q V^{5}}\right)^{1 / 4} .
$$

In the strong dissipative regime where $Q>>1$, the $\phi, N$-relation can be written

$$
\phi=\phi_{f}+\int_{0}^{N}\left(\frac{V, N}{Q V}\right)^{1 / 2} d N .
$$

Then

$$
\delta_{n s}=\frac{3}{4}\left(\ln \frac{Q^{2} V^{2}}{V, N}\right), N \quad, \quad r_{w}=8\left(\frac{4 C_{\gamma}}{9} \frac{V_{, N}^{3}}{Q^{6} V^{5}}\right)^{1 / 4} .
$$

Note that the only difference between the expressions for $\delta_{n s}$ and $r_{w}$ in terms of $N$ in the weak and strong regime is that $Q_{\text {weak }} \leftrightarrow Q_{\text {strong }}^{6}$. It follows from Equations (6.32.127) and (6.32.129) that the potential is in both cases given by

$$
V(N)=r_{W}^{4} \exp \left(4 \int \delta_{n s} d N\right) .
$$

Herrara [192] has considered a few examples. In the first one

$$
\delta_{n s}=\frac{2}{N} \quad, \quad r_{w}=\frac{1}{N(1+\xi N)} .
$$

In this case the $\delta_{n s}, r$-relation is

$$
r_{w}=\frac{\delta_{n s}^{2}}{2\left(2 \xi+\delta_{n s}\right)} .
$$

The observational result that $r_{W}<0.04=r_{\min }$ then leads to the requirement

$$
\xi>\frac{\delta_{n s}\left(\delta_{n s}-2 r_{\min }\right)}{4 r_{\min }} .
$$

Insering $\delta_{n s}=0.032$ gives $\xi>-0.0096$.

Equations (6.32.130) and (6.32.131) give the potential as a function of $N$

$$
V(N)=\frac{A}{(\xi+1 / N)^{4}},
$$

where A is a constant. Inserting this into Equation (6.32.126) gives in the weak regime

$$
N=\frac{1}{1-\xi \exp \left(-\frac{\phi-\phi_{0}}{4 A}\right)} .
$$


From the last two equations we have the potential

$$
V(\phi)=\frac{A}{\left[1+\xi\left(1-\exp \left(-\frac{\phi-\phi_{0}}{4 A}\right)\right)\right]^{4}} .
$$

In the strong regime Equations (6.32.128) and (6.32.129) lead to an integral that cannot be solved in terms of elementary functions.

In the special case that $\xi=0$ we have the prediction $\delta_{n s}=2 / N$ and $r_{w}=1 / N$, i.e., $r_{w}=\delta_{n s} / 2=0.016$. Equation (6.32.130) then gives $V(N)=K_{1} N^{4}$. Then we get from Equation (6.32.126) $N=\left[\left(\phi-\phi_{1}\right) / 4\right]^{2}$. Hence in this case the potential is

$$
V(\phi)=K_{2}\left(\phi-\phi_{1}\right)^{8} .
$$

The expressions for cold inflation corresponding to those in Equation (6.32.131) are found in Equation (6.1.42). For these models $r<0.04$ require a value of the exponent less than 0.3 , but warm inflation gives generally a smaller value of $r$ than the corresponding cold inflation models.

\subsection{Tachyon Inflation}

So called tachyon inflation is a string theory inspired model of inflation where the action has a certain form [28,61,198,199]. Although it has been shown by Kofman and Linde [200] that there are certain problems with tachyon inflation, it is considered here because it also has some nice properties. A recent analysis leading to observational constraints on tachyon inflation has been presented in [201].

\subsubsection{General Tachyon Inflation Equations}

In these models it has become usual to introduce a so-called tachyon field and denote it by $T$. A rolling tachyon field [202] can be described as a fluid which in the homogeneous limit has energy density and pressure

$$
\rho=\frac{V(T)}{\sqrt{1-\dot{T}^{2}}}, \quad p=-V(T) \sqrt{1-\dot{T}^{2}}=-\rho\left(1-\dot{T}^{2}\right),
$$

where $V(T)$ is the tachyon potential. Hence [194],

$$
w=p / \rho=-\left(1-\dot{T}^{2}\right)
$$

showing that the tachyon matter interpolates smoothly between LIVE for $\dot{T}=0$ and dust for $\dot{T}=1$.

The equation of motion of the tachyon field is

$$
\ddot{T}+\left(3 H \dot{T}+\frac{V_{T}}{V}\right)\left(1-\dot{T}^{2}\right)=0 .
$$

where we use the notation $V_{T} \equiv d V / d T$.

For this class of models Friedmann's first and second equations take the form

$$
H^{2}=\frac{\kappa}{3} \rho=\frac{\kappa}{3} \frac{V}{\sqrt{1-\dot{T}^{2}}} \quad, \quad \frac{\ddot{a}}{a}=H^{2}+\dot{H}=-\frac{\kappa}{6}(\rho+3 p)=\frac{\kappa}{3} \frac{V}{\sqrt{1-\dot{T}^{2}}}\left(1-\frac{3}{2} \dot{T}^{2}\right)
$$


There will be accelerated expansion when $\dot{T}^{2}<2 / 3$. Inserting Friedmann's first equation gives

$$
\dot{H}=-\frac{\kappa}{2} \frac{V \dot{T}^{2}}{\sqrt{1-\dot{T}^{2}}}=-\frac{3}{2} H^{2} \dot{T}^{2}
$$

Since $\dot{H}=H_{T} \dot{T}$ Equation (6.33.5) may also be written as

$$
\dot{T}=-\frac{2}{3} \frac{H_{T}}{H^{2}}
$$

It follows that [199]

$$
\dot{T}^{2}=1-\left(\frac{\kappa V}{3 H^{2}}\right)^{2}
$$

Inserting (6.33.7) into (6.33.6) gives

$$
H^{2} \dot{H}=\frac{\kappa^{2}}{6} V^{2}-\frac{3}{2} H^{4}
$$

Using Equation (6.33.7) this equation takes the form

$$
H_{T}^{2}=\frac{1}{4}\left(9 H^{4}-\kappa^{2} V^{2}\right)
$$

It follows from Equations (6.33.4) and (6.33.5) that Hubble slow roll parameters are

$$
\varepsilon_{H}=-\frac{\dot{H}}{H^{2}}=\frac{3}{2} \dot{T}^{2} .
$$

Hence a time independent tachyon field behaves as LIVE and gives exponential expansion, while integration of Equation (6.33.8) with a tachyon field having $\dot{T}=1$ gives $a \propto t^{2 / 3}$ which is the behavior of a dust dominated universe. As emphasi zed by Gibbons [194], if the tachyon condensate starts to roll down the potential with small initial value of $\dot{T}$, a universe dominated by this new form of matter will smoothly evolve from a phase of accelerated expansion to a phase dominated by a non-relativistic fluid.

It follows from Equations (6.33.4) and (6.33.5) that

$$
\frac{\ddot{a}}{a}=H^{2}\left(1-\frac{3}{2} \dot{T}^{2}\right)
$$

Hence, the condition for accelerated expansion, $\ddot{a}>0$, requires that $\dot{T}^{2}<2 / 3$. From Equation (6.33.9) we see that in terms of the Hubble slow roll parameter $\varepsilon_{H}$ the rate of change of the tachyon field is

$$
\dot{T}= \pm \sqrt{\frac{2 \varepsilon_{H}}{3}}
$$

Thus the tachyon field varies slowly during the slow roll era, meaning that $\dot{T}^{2}<<1, \ddot{T}<<3 H \dot{T}$. It then follows that Equations (6.33.3) and (6.33.4) reduce to

$$
3 H \dot{T} \approx-V_{T} / V, \quad H^{2} \approx \kappa V / 3
$$


Note that the first of these equations is different from the corresponding Equation (2.15) of the standard single field inflation. It follows from Equations (4.33.13) that

$$
\dot{T}=\frac{2}{\sqrt{3 \kappa}}\left(V^{-1 / 2}\right)_{T^{\prime}}
$$

which is different from Equation (2.17). Equations (6.33.10) and (6.33.14) give

$$
\varepsilon_{H}=\frac{1}{2 \kappa} \frac{V_{T}^{2}}{V^{3}}
$$

Inserting Equation (6.33.13) into (6.33.12) gives

$$
\ddot{a}=H^{2} a\left(1-\varepsilon_{H}\right) .
$$

This relation is exact. If $\varepsilon_{H}$ is neglected in Equation (4.33.12) so that $\ddot{a}=H^{2} a$, there is exponential expansion. The equation of state parameter $w$ may then be written

$$
w=-\left(1-\frac{2}{3} \varepsilon_{H}\right)
$$

showing that is has a value close to -1 .

The tachyon field is related to the usual inflaton field by

$$
\phi=\sqrt{V} T .
$$

Hence, during the inflationary era the derivative with respect to the inflaton field is related to the derivative with respect to the tachyon field by

$$
\frac{d}{d \phi}=\frac{1}{\sqrt{V}} \frac{d}{d T}
$$

which leads to

$$
V^{\prime}=\frac{V_{T}}{\sqrt{V}} \quad, \quad V^{\prime \prime}=\frac{V_{T T}}{V}-\frac{1}{2} \frac{V_{T}^{2}}{V^{2}} \quad, \quad V^{\prime \prime \prime}=\frac{V_{T}^{3}}{V^{7 / 2}}-2 \frac{V_{T} V_{T T}}{V^{5 / 2}}+\frac{V_{T T T}}{V^{3 / 2}} .
$$

Let us now introduce the horizon flow parameters defined in Equation (3.63), which gives

$$
\varepsilon_{1}=-\frac{H_{N}}{H^{2}} \quad, \quad \varepsilon_{2}=-\frac{d \ln \left|\varepsilon_{1}\right|}{d N} \quad, \quad \varepsilon_{2} \varepsilon_{3}=\frac{1}{\varepsilon_{1}} \frac{d^{2} \varepsilon_{1}}{d N^{2}}-\left(\frac{1}{\varepsilon_{1}} \frac{d \varepsilon_{1}}{d N}\right)^{2} .
$$

Here and in the following we use the notation $H_{N} \equiv d H / d N$ and so forth. Inserting these expressions into Equations (2.75) and (2.79) leads to

$$
\kappa \varepsilon_{1}=\frac{V_{T}^{2}}{2 V^{3}} \quad, \quad \kappa \varepsilon_{2}=3 \frac{V_{T}^{2}}{V^{3}}-2 \frac{V_{T T}}{V^{2}}=-\frac{2}{\sqrt{V}}\left(\frac{V_{T}}{V^{3 / 2}}\right)_{T} \quad, \quad \kappa^{2} \varepsilon_{2} \varepsilon_{3}=9 \frac{V_{T}^{4}}{V^{6}}-10 \frac{V_{T}^{2} V_{T T}}{V^{5}}+2 \frac{V_{T} V_{T T T}}{V^{4}} .
$$

It follows from Equations (3.1) and (3.26) in combination with the expressions (6.33.20), or alternatively from Equations (3.72) and (3.74) in combination with the expressions (6.33.22) that

$$
\eta=\frac{1}{\kappa}\left(\frac{V_{T T}}{V^{2}}-\frac{1}{2} \frac{V_{T}^{2}}{V^{3}}\right), \quad \eta_{H}=\frac{1}{\kappa}\left(\frac{V_{T T}}{V^{2}}-\frac{V_{T}^{2}}{V^{3}}\right) .
$$


On the other hand Fei et al. [75] have defined implicitely the potential slow roll parameter in the tachyon inflationary scenario by

$$
\kappa \eta_{V}=2 \frac{V_{T T}}{V^{2}} .
$$

Combining this with Equations (6.33.21) and (6.33.22) gives

$$
\eta_{V}=6 \varepsilon_{1}+\left(\ln \varepsilon_{1}\right)_{N} .
$$

From Equation (3.51) with $\varepsilon=\varepsilon_{1}$ and Equation (6.33.19) we have

$$
V_{T}=\sqrt{\frac{\kappa V}{2 \varepsilon_{1}}} V_{N} .
$$

Inserting this into the expression for $\varepsilon_{1}$ in Equation (6.20.20) gives

$$
\varepsilon_{1}=\frac{1}{2} \frac{V_{N}}{V} .
$$

which requires that $V_{N}>0$. Integration of Equation (6.33.27) gives

$$
V(N)=V_{0} \exp \left[2 \int \varepsilon_{1} d N\right] .
$$

From Equations (3.63) and (4.56) we have to lowest order

$$
\delta_{n s}=2 \varepsilon_{1}-\left(\ln \varepsilon_{1}\right)_{N} \quad, \quad r=16 \varepsilon_{1} \quad, \quad \alpha_{S}=\left(\delta_{n s}\right)_{N} .
$$

Inserting Equation (6.33.27) into the first of the expressions (6.33.29) we obtain

$$
\delta_{n s}=\left(\ln \frac{V^{2}}{V_{N}}\right)_{N} .
$$

It follows from Equations (6.33.22) and (6.33.14) that the two first horizon-flow parameters are

$$
\varepsilon_{1}=\frac{3}{2} \dot{T}^{2} \quad, \quad \varepsilon_{2}=\frac{2 \ddot{T}}{H \dot{T}} .
$$

Steer and Vernizzi [28] have shown that to 2. order in the low roll parameters we have the consistency relationship

$$
n_{T}=-\frac{r}{8}\left(1+\delta_{n s}-\frac{r}{24}\right) .
$$

which replaces the corresponding relation (4.16) in standard single field inflation.

We shall now express the optical parameters in terms of the number of e-folds. Using the first of the Equation (6.33.22), the Equation (3.50) of the standard single field inflation is replaced by

$$
T_{N}=M_{P}^{2} \frac{V_{T}}{V^{2}} .
$$

Combing Equation (6.33.1) with (6.33.26) and (6.33.27) we obtain

$$
T_{N}=\sqrt{2} M_{P} \sqrt{\frac{\varepsilon_{1}}{V}} .
$$


Hence the tachyon field is given in terms of the number of e-folds as

$$
T(N)=\sqrt{2} M_{P} \int \sqrt{\frac{\varepsilon_{1}}{V}} d N .
$$

Note also that it follows from Equation (6.33.26) that the relationship between the tachyon field and the number of e-folds may also be written as

$$
N(T)=\frac{1}{M_{P}^{2}} \int_{T}^{T_{f}} \frac{V^{2}}{V_{T}} d T,
$$

When combining Equations (6.33.26) and (6.33.14), we obtain

$$
V_{T}=\sqrt{\frac{3}{2}} \frac{H}{\sqrt{\varepsilon_{1}}} V_{N} .
$$

Hence, an alternative form of Equation (6.33.36) is

$$
N(T)=\sqrt{\frac{3}{2}} \int_{T}^{T_{f}} \frac{H}{\sqrt{\varepsilon_{1}}} d T .
$$

Sometimes one needs to calculate the relationship between the tachyon inflaton field $T$ and the number of e-folds $N$ from a knowledge of the potential $V$ as a function of $N$. This may be obtained from Equations (6.33.26), (6.33.27), and (6.33.33) that lead to the relationship

$$
\frac{d T}{d N}=M_{P} \frac{\sqrt{V_{N}}}{V} .
$$

or

$$
T=M_{P} \int \frac{\sqrt{V_{N}}}{V} d N .
$$

We shall now review several types of tachyon inflation models.

6.33.2. Tachyon Inflation with Constant Value of $\varepsilon_{2}$

Q. Fei et al. [75] have considered a tachyon inflation model with constant value of $\varepsilon_{2}$. It then follows from Equation (6.33.21) that

$$
\varepsilon_{1}=e^{-\varepsilon_{2} N} .
$$

It follows from Equations (6.33.29) and (6.33.41) that

$$
\delta_{n s}=\frac{r}{8}-\frac{1}{N} \ln \left(\frac{r}{16}\right) .
$$

This function has a minimum at $r_{1}=8 / N$ with

$$
\delta_{n s}\left(r_{1}\right)=\frac{1+\ln (2 N)}{N} .
$$

Inserting $N=60$ gives $\delta_{n s}=0.096$ which is about three times larger than the value favored by the Planck data. Hence the model with constant value of $\varepsilon_{2}$ is excluded by the Planck data. 
6.33.3. Tachyon Inflation with Constant Value of $\delta_{n s}$

Fei et al. then considered a model with constant value of $\delta_{n s}$. Integration of Equation (4.20.31) with $\varepsilon_{1}(0)=1$ then gives

$$
r=\frac{16 \delta_{n s}}{\left(2-\delta_{n s}\right) e^{N \delta_{n s}}-2} \approx \frac{8 \delta_{n s}}{e^{N \delta_{n s}-1}} .
$$

Inserting the Planck value $\delta_{n s}=0.032$ and $N=60$ gives $r=0.044$ which is just at the upper limit of what is acceptable according to the present observational data.

6.33.4. Tachyon Inflation with Constant Value of $\eta_{H}$

Furthermore, Fei et al. considered a tachyon inflation model with constant Hubble slow motion parameter $\eta_{H}$. Equation (3.59) can be written

$$
\eta_{H}=\varepsilon_{H}+(1 / 2)\left(\ln \varepsilon_{H}\right){ }_{N} \cdot
$$

Integrating this for constant $\eta_{H}$ and with $\varepsilon_{H}(0)=1$ gives

$$
\varepsilon_{H}=\frac{\eta_{H}}{1-\left(1-\eta_{H}\right) e^{-2 \eta_{H} N}}
$$

Using this expression together with the Equation (4.20) leads to

$$
\delta_{n s}=\frac{4 \eta_{H}}{1-\left(1-\eta_{H}\right) e^{-2 \eta_{H} N}}-2 \eta_{H} \quad, \quad r=\frac{16 \eta_{H}}{1-\left(1-\eta_{H}\right) e^{-2 \eta_{H} N}} .
$$

Hence

$$
\eta_{H}=\frac{1}{8}\left(r-4 \delta_{n s}\right) .
$$

Inserting this into the last of the Equation (4.33.47) gives

$$
N=\frac{4}{r-4 \delta_{n s}} \ln \frac{r\left(8-r+4 \delta_{n s}\right)}{8\left(8 \delta_{n s}-r\right)} .
$$

In Figure 10 we have inserted $\delta_{n s}=0.032$ and plotted $N$ as a function of $r$.

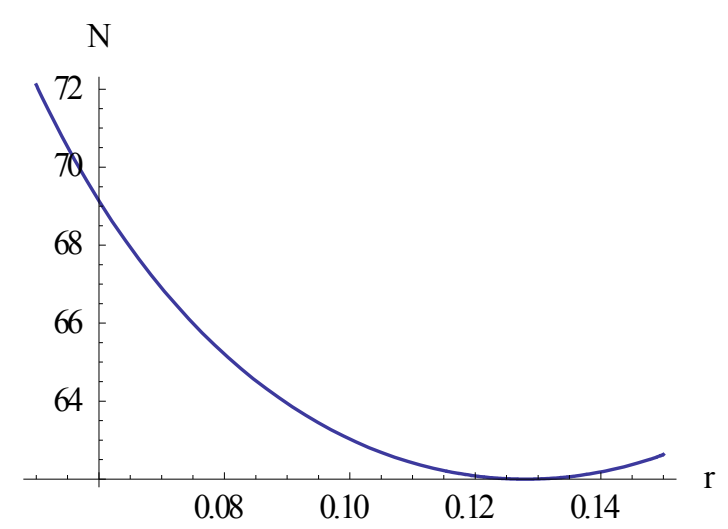

Figure 10. The number of e-folds $N$ as given in Equation (6.33.37) plotted as function of $r$.

We see that $N$ has a minimum for $r \approx 0.127$ with $N_{\min }=62$, so this model is marginally in agreement with the Planck data. 
6.33.5. Tachyon Inflation with Constant Value of $\eta_{V}$

Fei et al. also considered a tachyon inflation model with constant potential slow motion parameter $\eta_{V}$. Integrating Equation (6.33.31) then gives

$$
\varepsilon_{1}=\frac{\eta_{V}}{2-\left(2-\eta_{V}\right) e^{-2 \eta_{V} N}}
$$

Inserting this into Equation (4.13) gives

$$
\delta_{n s}=\frac{6 \eta_{V}}{2-\left(2-\eta_{V}\right) e^{-2 \eta N}}-2 \eta_{V} \quad, \quad r=\frac{16 \eta_{V}}{2-\left(2-\eta_{V}\right) e^{-2 \eta_{V} N}}
$$

Hence

$$
\eta_{V}=\frac{1}{16}\left(3 r-8 \delta_{n s}\right)
$$

With $\delta_{n s}=0.032$ a positive value of $\eta_{V}$ requires $r>0.085$. Inserting the expression (6.33.49) into the second of expressions (6.33.51) leads to

$$
N=\frac{8}{3 r-8 \delta_{n s}} \ln \frac{r\left(32+8 \delta_{n s}-3 r\right)}{16\left(8 \delta_{n s}-r\right)} .
$$

In Figure 11 we have inserted $\delta_{n s}=0.032$ and plotted $N$ as a function of $r$.

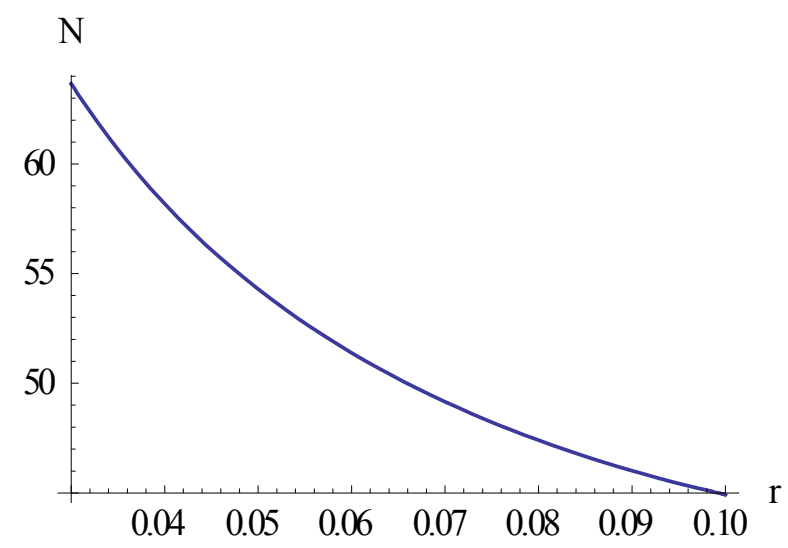

Figure 11. The number of e-folds $N$ as given in Equation (6.33.53) plotted as function of $r$.

We see that in order to have $50<N<60$ the tensor to scalar ratio must obey $0.036<r<0.065$ which is marginally in agreement with the Planck data.

Inserting the expression (6.33.51) for $r$ into Equation (3.55) and integrating gives

$$
V(N)=\sqrt{V(0)^{2}+2\left(e^{2 \eta_{V} N}-1\right)}
$$

where $V(0)=\sqrt{\eta_{V}}$.

\subsubsection{Self-Dual Tachyon Inflation}

D. A. Steer and F. Vernizzi, [28] and also Rezazadeh, Karami and Hashemi [173] have considered a tachyon inflation model with the same potential (6.8.1) as in the S-dual model and used the horizon-flow parameters to calculate the scalar spectral index, its running and the tensor to scalar ratio. The potential is written

$$
V(T)=\frac{V_{0}}{\cosh \hat{T}^{\prime}}
$$


where $V_{0}=V(0)$ and $\hat{T}=T / T_{0}$. The tachyon field has approximately the field strength $T_{0}$ during the inflationary era. From Equation (6.33.22) we then get

$$
\kappa \varepsilon_{1}=\frac{1}{2 V_{0} T_{0}^{2}} \frac{\sinh ^{2} \hat{T}}{\cosh \hat{T}}, \quad \kappa \varepsilon_{2}=\frac{1}{V_{0} T_{0}^{2}} \frac{1+\cosh ^{2} \hat{T}}{\cosh \hat{T}} \quad, \quad \kappa^{2} \varepsilon_{2} \varepsilon_{3}=\frac{1}{V_{0}^{2} T_{0}^{4}} \frac{\sinh ^{4} \hat{T}}{\cosh ^{2} \hat{T}^{\prime}}
$$

Inserting these expressions into those in Equation (4.56), we obtain

$$
\delta_{n s}=\frac{2}{V_{0} T_{0}^{2}} \cosh \hat{T} \quad, \quad r=\frac{8}{V_{0} T_{0}^{2}} \frac{\sinh ^{2} \hat{T}}{\cosh \hat{T}} \quad, \quad \alpha_{S}=-\frac{2}{V_{0}^{2} T_{0}^{4}} \sinh ^{2} \hat{T} .
$$

These expressions are different from those in Equation (6.8.6) for the same potential in standard single field inflation. It follows from the expressions for $\delta_{n s}$ and $r$ that

$$
V_{0} T_{0}^{2}=\frac{4}{\sqrt{\left(4 \delta_{n s}-r\right) \delta_{n s}}} .
$$

Inserting the BPK-values $\delta_{n s}=0.32, r<0.04$ and $r_{\min }=0$, corresponding to $\left(V_{0} T_{0}^{2}\right)_{\min }=2 / \delta_{n s}$, gives $62.5<V_{0} T_{0}^{2}<75$. Furthermore

$$
\alpha_{S}=-(1 / 8) r \delta_{n s}
$$

Hence $\left|\alpha_{S}\right|<0.0005$ showing that the running of the scalar spectral index is very small in this inflationary model.

Inserting the potential (6.33.55) into Equation (6.33.34) gives the number of e-folds

$$
N(T)=V_{0} T_{0}^{2} \int_{\hat{T}}^{\hat{T}_{f}} \frac{d \hat{T}}{\sinh \hat{T}}=V_{0} T_{0}^{2} \ln \frac{\tanh \left(\hat{T}_{f} / 2\right)}{\tanh (\hat{T} / 2)} .
$$

The end of the inflationary era takes place when $\varepsilon_{1}\left(T_{f}\right)=1$, giving

$$
\cosh \hat{T}_{f}=V_{0} T_{0}^{2}+\sqrt{V_{0}^{2} T_{0}^{4}+1}
$$

To 2. order in $\left(1 / 2 V_{0} T_{0}^{2}\right)$ this gives

$$
\tanh \left(\frac{\hat{T}_{f}}{2}\right)=1+\frac{3}{2}\left(\frac{1}{2 V_{0} T_{0}^{2}}\right)^{2}
$$

Hence we can use the approximation $\tanh \left(\hat{T}_{f} / 2\right)=1$ in Equation (6.33.58), giving $\ln \left[\tanh \left(\hat{T}_{f} / 2\right)\right] \approx \ln 1=0$, which leads to

$$
\tanh \left(\frac{\hat{T}}{2}\right)=e^{-\frac{N}{V_{0} T_{0}^{2}}}
$$

It follows that

$$
\sinh \hat{T}=\frac{1}{\sinh \left(N / V_{0} T_{0}^{2}\right)} \quad, \quad \cosh \hat{T}=\operatorname{coth}\left(N / V_{0} T_{0}^{2}\right) .
$$


Inserting these expressions into Equation (6.33.57) gives

$$
\delta_{n s}=\frac{2}{V_{0} T_{0}^{2}} \operatorname{coth}\left(\frac{N}{V_{0} T_{0}^{2}}\right) \quad, \quad r=\frac{16}{V_{0} T_{0}^{2}} \frac{1}{\sinh \left(2 N / V_{0} T_{0}^{2}\right)} \quad, \quad \alpha_{S}=-\frac{2}{V_{0}^{2} T_{0}^{4}} \frac{1}{\sinh ^{2}\left(N / V_{0} T_{0}^{2}\right)} \text {. }
$$

\subsubsection{Exponential Tachyon Inflation}

Steer and F. Vernizzi, [28] and Rezazadeh, Karami and Hashemi [173] have also considered a tachyon inflation model with exponential potential

$$
V(T)=V_{0} e^{-\hat{T}} .
$$

The corresponding model in standard inflation was considered in Section 4.4 and then leads to power law inflation as given in Equation (4.4.7), and a tensor-to-scalar ratio that is larger than allowed by the Planck data. However in the Tachyon inflation scenario the model with this potential give more interesting predictions, as will be shown below.

Inserting the potential (6.33.66) into Equation (6.33.15) and integrating with $T_{0}=T\left(t_{0}\right)$ gives the time dependency of the tachyon potential

$$
e^{-\hat{T}}=\left(e^{-\hat{T}_{0} / 2}+\frac{t-t_{0}}{2 \sqrt{3 V_{0}}}\right)^{2}
$$

where $t_{0}$ is a positive constant. The potential has a power law time dependence

$$
V(t)=V_{0}\left(e^{-\hat{T}_{0} / 2}+\frac{t-t_{0}}{2 \sqrt{3 V_{0}}}\right)^{2} .
$$

Hence, according to the slow roll approximation (6.33.12) the Hubble parameter is.

$$
H(t)=\sqrt{\frac{\kappa V_{0}}{3}}\left(e^{-\hat{T}_{0} / 2}+\frac{t-t_{0}}{2 \sqrt{3 V_{0}}}\right) .
$$

The first Hubble slow roll parameter, given in Equation (6.33.11), is

$$
\varepsilon_{H}=\frac{1}{2 V_{0}} e^{\hat{T}} .
$$

The slow roll era ends when $\varepsilon_{H}\left(\hat{T}_{f}\right)=1$ giving

$$
e^{\hat{T}_{f}}=2 V_{0}
$$

Inserting the potential (6.33.66) into Equation (6.33.38) and performing the integration gives

$$
N=V_{0}\left(e^{-\hat{T}}-e^{-\hat{T}_{f}}\right) .
$$

The last three equations together with Equation (6.33.22) give

$$
2 \varepsilon_{1}=\varepsilon_{2}=\varepsilon_{3}=\frac{2}{2 N+1} .
$$

Inserting this into Equation (4.56) we

$$
\delta_{n s}=\frac{4}{2 N+1} \quad, \quad r=\frac{16}{2 N+1} \quad, \quad \alpha_{S}=-\frac{8}{(2 N+1)^{2}} .
$$


Inserting $\delta_{n s}=0.032$ gives $N=62$ in good agreement with the inflationary requirements. The expressions (6.33.71) imply $r=4 \delta_{n s}=0.128$ which is too large to be compatible with the Planck data. Furthermore $\alpha_{s}=-(1 / 2) \delta_{n s}^{2}=-0.0005$. Note the agreement with the tachyonic inflation with exponential inflation and the prediction (6.1.37) of standard inflation with power law inflation having $p=2$-the so-called chaotic inflation.

\subsubsection{Inverse Power Law Tachyon Inflation}

Rezazadeh, Karami and Hashemi [173] have also considered tachyon inflation with inverse power-law potential,

$$
V(T)=V_{0} \hat{T}^{-n} .
$$

Inserting this into Equation (6.33.13) and integrating with $T\left(t_{P}\right)=M_{P}$ and $n \neq 4$ gives

$$
\hat{T}(t)=\left[1+A\left(t-t_{P}\right)\right]^{\frac{2}{4-n}} \quad, \quad A=\frac{n(4-n) M_{P}^{\frac{n}{2}-1}}{2 \sqrt{3 V_{0} T_{0}^{n}}} .
$$

Hence

$$
V(t)=V_{0}\left[1+A\left(t-t_{P}\right)\right]^{-\frac{2 n}{4-n}} .
$$

Inserting this into Equation (6.33.4) and neglecting $\dot{T}$ compared to 1 in the slow roll era, we get

$$
H(t)=\frac{1}{M_{P}} \sqrt{\frac{V_{0}}{3}}\left[1+A\left(t-t_{P}\right)\right]^{-\frac{n}{4-n}} .
$$

Normalizing the scale factor to $a\left(t_{P}\right)=1$ gives

$$
a(t)=\exp \left\{\frac{1}{M_{P} A} \sqrt{\frac{V_{0}}{3}}\left[1+A\left(t-t_{P}\right)\right]^{\frac{2(2-n)}{4-n}}-1\right\}
$$

It follows from Equations (6.33.15) and (6.33.75) that the first Hubble slow roll parameter is

$$
\varepsilon_{H}=\frac{n^{2}}{2 V_{0} \hat{T}_{0}^{2}} \hat{T}^{n-2} .
$$

For $n=2$ the slow roll parameter $\varepsilon_{H}$ is constant, and then there is no graceful exit from the slow roll era. Furthermore Rezazadeh, Karami and Hashemi [173] have shown that this model is ruled out by the Planck data. The inflaton field increases with time for $n<4$. A graceful exit of the slow roll era requires that $\varepsilon_{H}$ increases with time and hence that $2<n<4$.

Inserting the potential (6.33.73) into Equation (6.33.41) and performing the integration gives

$$
N=\frac{V_{0} T_{0}^{2}}{n(n-2)}\left(\hat{T}^{2-n}-\hat{T}_{f}^{2-n}\right) .
$$

The slow roll era ends when $\varepsilon_{H}\left(T_{f}\right)=1$, giving

$$
T_{f}^{2-n}=\frac{n^{2}}{2 V_{0} \hat{T}_{0}^{2}} .
$$

Hence

$$
\hat{T}^{2-n}=\frac{n[2(n-2) N+n]}{2 V_{0} T_{o}^{2}} .
$$


Inserting this into Equation (6.33.80) gives

$$
\varepsilon_{1}=\varepsilon_{H}=\frac{n}{(n-2) N+n} .
$$

From Equation (6.33.28) we now obtain

$$
\delta_{n s}=\frac{4(n-1)}{2(n-2) N+n} \quad, \quad r=\frac{16 n}{2(n-2) N+n} \quad, \quad \alpha_{S}=-\frac{8(n-1)(n-2)}{[2(n-2) N+n]^{2}} .
$$

These expressions imply that

$$
r=\frac{4 n}{n-1} \delta_{n s}>4 \delta_{n s} .
$$

With $\delta_{n s}=0.032$ this relationship gives $r>0.128$ while the BPK-data require $r<0.04$, so this model is not in agreement with the observational data.

\subsubsection{Tachyon-Intermediate Inflation}

S. del Campo, R. Herrera and A. Toloza [203] have considered the intermediate inflation in the tachyonic framework. Then the scale factor is given in Equation (6.30.1). The Hubble parameter and its derivatives with respect to cosmic time are given in Equation (6.30.2). However Equation (2.1) is now replaced by (6.33.1) so the density and pressure of the tachyonic field are not given by Equation (6.30.3).

Inserting the expressions (6.30.2) into Equation (6.33.6) and integrating with $T(0)=0$ gives

$$
T=\frac{2}{2-\alpha} \sqrt{\frac{2(1-\alpha)}{3 \alpha}} \hat{t}^{\frac{2-\alpha}{2}},
$$

which replaced Equation (5.30.5) in the ordinary intermediate inflation. It is assumed that $0<\alpha<1$. Hence the tachyon field is an increasing function of time. From Equations (6.33.4) and (6.33.6) we obtain

$$
\kappa V=H \sqrt{3\left(3 H^{2}+2 \dot{H}\right)} .
$$

Inserting the expressions (6.30.2) gives

$$
\kappa^{2} V(t)=3 \alpha^{2} \hat{t}^{-2(1-\alpha)} \sqrt{1-\frac{2(1-\alpha)}{3 \alpha} \hat{t}^{-\alpha}} .
$$

It follows from Equations (6.33.84) and (6.33.86) that the potential as a function of the tachyon field is

$$
\begin{aligned}
& \kappa^{2} V=A_{1} T^{-2 \beta} \sqrt{1-A_{2} T^{-2(1-\beta)}}, \quad \beta=\frac{2(1-\alpha)}{2-\alpha} \\
& A_{1}=3 A^{2} \alpha^{2}\left[\frac{(2-\beta)^{2} \beta}{3(1-\beta)}\right]^{\beta}, \quad A_{2}=\left\{(2-\beta)^{\alpha}\left[\frac{\beta}{3(1-\beta)}\right]\right\}^{2-\beta} \\
& \varepsilon_{H}=\frac{\beta}{2(1-\beta)}\left[\frac{\beta(1-\beta)}{3}\right]^{1-\beta} T^{-2(1-\beta)}, \quad \eta_{H}=\frac{1}{\beta} \varepsilon_{H}
\end{aligned}
$$

The Hubble slow roll parameters are given as a function of time in Equation (6.30.9). Combining this with Equation (6.33.88) leads to

$$
\varepsilon_{H}=\frac{\beta}{2(1-\beta)}\left[\frac{\beta(1-\beta)}{3}\right]^{1-\beta} T^{-2(1-\beta)}, \quad \eta_{H}=\frac{1}{\beta} \varepsilon_{H} .
$$


Hence $\varepsilon_{H}$ is a decreasing function of time. So like standard intermediate inflation there is no natural finish of the slow roll era, and the parameter $\varepsilon_{H}$ is used to define the initial value of the inflaton field by the condition $\varepsilon_{H}\left(T_{i}\right)=1$, giving

$$
T_{i}^{2(1-\beta)}=\frac{\beta}{2(1-\beta)}\left[\frac{\beta(1-\beta)}{3}\right]^{1-\beta} .
$$

When combining Equations (6.30.11) and (6.33.84) we find that the number of e-folds during the inflationary era is

$$
N=\left[(2-\beta) \sqrt{\frac{3(1-\beta)}{\beta}}\right]^{2(1-\beta)}\left[T^{2(1-\beta)}-T_{f}^{2(1-\beta)}\right] .
$$

The tachyon inflaton potential is an increasing function of $N$. Inserting this into Equation (6.33.91) gives

$$
T^{2(1-\beta)}=\frac{\beta}{2(1-\beta)}\left[\frac{\beta(1-\beta)}{3}\right]^{1-\beta}\left\{1+2(1-\beta)[(1-\beta)(2-\beta)]^{-2(1-\beta)} N\right\} .
$$

Hence

$$
\varepsilon_{H}=\frac{[(1-\beta)(2-\beta)]^{2(1-\beta)}}{2(1-\beta) N+[(1-\beta)(2-\beta)]^{2(1-\beta)}} .
$$

In tachyon inflation, the scalar spectral index is not given in the same way as in standard inflation in terms of the Hubble parameters. However, Rezazadeh et al. [173] have shown that the $r$, $\delta_{n s}-$ relation is

$$
r=\frac{8 \beta}{2 \beta-1} \delta_{n s} .
$$

It follows from this relationship that $r>4 \delta_{n s}=0.128$. Hence these inflationary models are ruled out by the BPK-data which according to Gott and Colley [46], require $r<0.04$.

\subsubsection{The N-Formalism Applied to Tachyon Inflation}

N. Barbosa-Cendejas et al. $[61,201]$ have used the $N$-formalism and the horizon-flow parameters, and studied a tachyon inflationary model with $n=1$ of the perturbative class considered in Section 5.2. Then Equation (3.73) takes the form

$$
\varepsilon_{1}=\frac{\beta}{N} .
$$

Inserting this into Equation (6.33.94) and performing the integration gives

$$
V(N)=V_{0}\left(\frac{N}{N_{0}}\right)^{2 \beta}
$$

Substituting this into Equation (6.33.33) and integrating with $T(0)=0$ shows that the potential is

$$
V(T) \propto\left\{\begin{array}{lc}
V_{0}\left(\frac{T}{T_{0}}\right)^{\frac{4 \beta}{1-2 \beta}}, \quad \beta \neq 1 / 2 \\
V_{0} e^{\frac{1}{M_{P}} \sqrt{\frac{V_{0}}{2 \beta N_{0}}}\left(T-T_{0}\right)}, \quad \beta=1 / 2
\end{array} .\right.
$$

From Equations (6.33.97) and (6.33.25) we get

$$
\delta_{n s}=\frac{1+2 \beta}{N}, \quad r=\frac{16 \beta}{N}, \quad \alpha_{S}=-\frac{1+2 \beta}{N^{2}}
$$


This gives

$$
\beta=\frac{1}{2}\left(N \delta_{n s}-1\right) \quad, \quad r=8\left(\delta_{n s}-\frac{1}{N}\right) .
$$

When comparing with Equation (6.1.44) we see that the last of these equations is the same consistency relationship as that for ordinary polynomial inflation. With $N=50, \delta_{n s}=0.032$ we get $\beta=0.3, r=0.096$. This value of the tensor-to-scalar ratio is larger than allowed by the most recent analysis of the observational data [46], $r<0.04$.

Fei et al. [75] have considered a model with

$$
\delta_{n s}=\frac{p}{N+A}
$$

Inserting this into Equation (6.33.26) gives

$$
\left(\ln \frac{V^{2}}{V_{N}}\right)_{N}=\frac{p}{N+A}
$$

The solution of this equation with $V_{0}=V(0)$ is

$$
V(N)=\left\{\begin{array}{ll}
\frac{V_{0}}{1+C_{1}\left[(N+A)^{1-p}-A^{1-p}\right]}, & p \neq 1 \\
\frac{V_{0}}{1+C_{2} \ln \left(1+\frac{N}{A}\right)} & , \quad p=1
\end{array},\right.
$$

where $C_{1}$ and $C_{2}$ are constants. Inserting this into Equation (6.20.32) gives

$$
\varepsilon_{1}=\left\{\begin{array}{ll}
\frac{C_{1}(p-1)}{2(N+A)\left[C_{1}+\left(A^{p-1}-C_{1}\right)\left(1+\frac{N}{A}\right)^{p-1}\right]}, & p \neq 1 \\
\frac{C_{2}}{2(N+A)\left[1+C_{2} \ln \left(1+\frac{N}{A}\right)\right]} & , \quad p=1
\end{array} .\right.
$$

The constants are determined by the condition that the slow roll era ends when $\varepsilon_{1}(0)=1$, giving

$$
C_{1}=\frac{2 A^{p}}{p-1} \quad, \quad C_{2}=-2 A .
$$

Hence the potential and the first horizontal slow roll parameter are

$$
V(N)= \begin{cases}\frac{V_{0}}{1+\frac{2 A}{p-1}\left[\left(1+\frac{N}{A}\right)^{1-p}-1\right]}, & p \neq 1 \\ \frac{V_{0}}{1-2 A \ln \left(1+\frac{N}{A}\right)} & , \quad p=1\end{cases}
$$

and

$$
\varepsilon_{1}=\left(\begin{array}{ll}
\frac{p-1}{2(N+A)+(p-1-2 A)\left(1+\frac{N}{A}\right)^{p}} & , \quad p \neq 1 \\
\frac{1}{\left(1+\frac{N}{A}\right)\left[1-2 A \ln \left(1+\frac{N}{A}\right)\right]} & , \quad p=1
\end{array}\right.
$$

Combining this with Equation (6.33.107) we get the $\delta_{n s}, r-$ relations

$$
r= \begin{cases}\frac{16\left[(N+A) \delta_{n s}-1\right]}{2(N+A)+\left[(N+A) \delta_{n s}-1-2 A\right]\left(1+\frac{N}{A}\right)^{(N+A) \delta_{n s}}}, & p \neq 1 \\ \frac{A \delta_{n s}}{1+2 A \ln \left(A \delta_{n s}\right)}, & , \quad p=1\end{cases}
$$

The tensor-to-scalar ratio $r$ is plotted as function of $A$ from the expression (6.33.109) for $p \neq 1$ with $\delta_{n s}=0.032$ and $N=50$ in Figure 12 . 
The factor $(N+A) \delta_{n s}-1-2 A$ changes sign for

$$
A=A_{1}=\frac{N \delta_{n s}-1}{2-\delta_{n s}} .
$$

Inserting $N=50$ and $\delta_{n s}=0.032$ gives $A_{1}=0.31$ while $N=60$ gives $A_{1}=0.45$. Because $\left(1+\frac{N}{A_{1}}\right)^{\left(N+A_{1}\right) \delta_{n s}} \approx 3 \times 10^{3}$ is so large, $r$ changes sign close to $A=A_{1}$. Since only positive values of $r$ are allowed, this class of models require that $A<A_{1}$. This is the reason of the restricted range of $A$ in Figure 12.

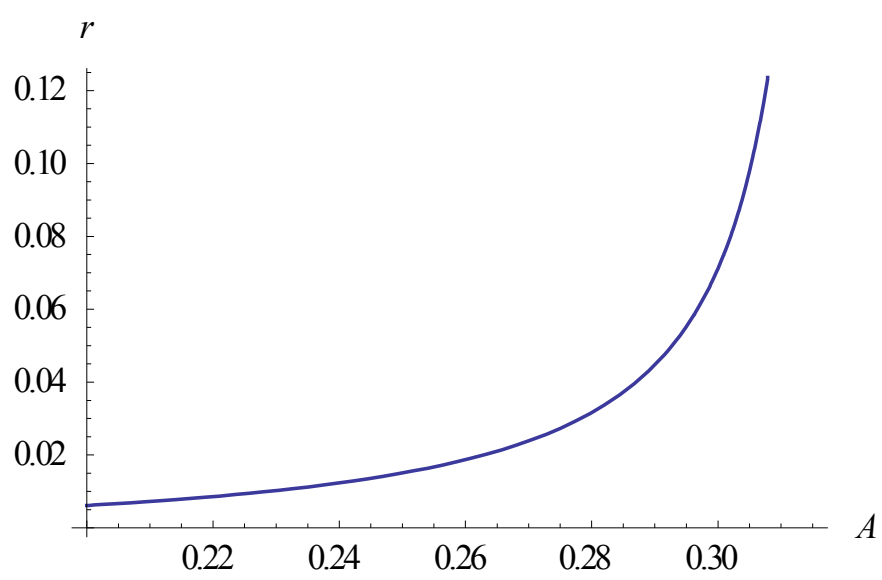

Figure 12. The tensor to scalar ratio as given in Equation (6.33.111) for $p \neq 1$ plotted as function of $A$ in the region $0.20<A<0.315$ for $N=50$ and $\delta_{n s}=0.032$.

It may be noted that

$$
r\left(A_{1}\right)=\frac{16\left(N \delta_{n s}-1\right)}{2 N-1} \approx 8\left(\delta_{n s}-\frac{1}{N}\right)
$$

With $N=60, \delta_{n s}=0.032$ gives $r\left(A_{1}\right) \approx 0.12$.

Equation (6.33.103) can be written as

$$
A=\frac{p}{\delta_{n s}}-N \quad \text { or } \quad p=(N+A) \delta_{n s} .
$$

Hence $A>0$ requires $p>N \delta_{n s}$. Inserting $N=50, \delta_{n s}=0.032$ gives $p>1.6$. The value $p=1$ gives $A=\left(1 / \delta_{n s}\right)-N$. With $N>50, \delta_{n s}=0.032$ we have $A<-19$. However negative values of $A$ are not allowed by the expression (6.33.109) for $p=1$. So the model with $p=1$ is excluded by the Planck data.

The relationship between the tachyon inflaton potential and the number of e-folds of the scale factor during the slow roll era can be calculated by inserting Equation (6.33.107) into Equation (6.33.35). Performing the integration with $T_{0}=T(0)$ one obtains for $p \neq 1, p \neq 2$,

$$
N=A\left[1+\beta_{1}\left(T-T_{0}\right)\right]^{\frac{2}{2-p}}-A \quad, \quad \beta_{1}=\frac{(2-p) \sqrt{V_{0} / 2}}{2 A M_{P}} .
$$

Inserting this into Equation (6.33.107) one obtains the inverse power law potential

$$
V(T)=\frac{V_{0}}{1+\frac{2 A}{p-1}\left\{\left[1+\beta_{1}\left(T-T_{0}\right)\right]^{\frac{2(1-p)}{2-p}}-1\right\}} .
$$

In the special case with $p=p_{1}$ and $A=A_{1}$ the potential takes the form 


$$
V(T)=V_{0}\left[1+\beta_{1}\left(T-T_{0}\right)\right]^{\frac{2(p-1)}{2-p}} .
$$

For $p=2$ the potential as given in Equation (6.33.107) takes the form

$$
V(N)=\frac{V_{0}(N+A)}{A+(1-2 A) N} .
$$

Inserting this equation into Equation (6.33.32) and integrating with $N\left(T_{0}\right)=0$ gives

$$
N=A\left[e^{\left.\sqrt{V_{0} / 2\left(1 / A M_{P}\right)\left(T-T_{0}\right)}-1\right]} .\right.
$$

Substituting this into Equation (6.33.116) gives

$$
V(T)=\frac{V_{0}}{1+2 A\left[e^{-\sqrt{V_{0} / 2}\left(1 / A M_{P}\right)\left(T-T_{0}\right)}-1\right]} .
$$

The expression reduces to the exponential potential

$$
V=V_{0} e^{\sqrt{2 V_{0}}\left(T-T_{0}\right) / M_{P}}
$$

if $A=1 / 2$. In this case Equation (6.33.102) gives $N=\left(4-\delta_{n s}\right) / 2 \delta_{n s}=62$ which is an acceptable value of $N$. Inserting these values into the first expression in Equation (6.33.109) gives $r=0.064$. Hence the tachyonic inflationary model with an exponential potential is allowed by the observational data, while in the standard inflationary scenario the model with exponential potential gives too high value of $r$ as seen in Equation (6.4.12).

Fei et al. [75] have also considered a class of tachyonic inflationary models with the first horizontal slow roll parameter given by

$$
\varepsilon_{1}=\frac{1}{\left(1+N / N_{0}\right)^{p}},
$$

where I have used the notation of Equation (5.66). The $\delta_{n s}, r$-relationship is the same as Equation (5.67) in the standard inflationary scenario, and a prediction of this class of inflationary models is that $r<0.033$. However, the expressions of the potential are different in the tachyonic inflationary scenario.

We can use the Planck data to estimate realistic values of $p$ and $N_{0}$. The scalar tilt and the tensor-to-scalar ratio are

$$
\delta_{n s}=\frac{2}{\left(1+\frac{N}{N_{0}}\right)^{p}}+\frac{p}{N+N_{0}} \quad, \quad r=\frac{16}{\left(1+\frac{N}{N_{0}}\right)^{p}} .
$$

From these equations it follows that the $\delta_{n s}, r$-relation has the form

$$
\delta_{n s}=\frac{r}{8}+\frac{p}{N+N_{0}}=\frac{r}{8}+\frac{p}{N_{0}}\left(\frac{r}{16}\right)^{1 / p} .
$$

We can now express $\mathrm{p}$ and $N_{0}$ in terms of $\delta_{n s}, r$ and $N$ as

$$
\left[1-\left(\frac{r}{16}\right)^{1 / p}\right] p=N\left(\delta_{n s}-\frac{r}{8}\right) \quad, \quad N_{0}=\frac{8 p}{8 \delta_{n s}-r}-N .
$$

Inserting $\delta_{n s}=0.032, r=0.05, N=60$, solving the first equation numerically for $p$, and inserting the result into the second equation, gives $p=1.6, N_{0}=2$. Hence these are realistic values for this class of tachyonic inflationary models. 
Substituting Equation (6.33.120) into Equation (6.33.27) and integrating gives

$$
V(N)=\left\{\begin{array}{ll}
V_{0} e^{\frac{2}{1-p}\left(N+N_{0}\right)}, & p \neq 1 \\
V_{0}\left(1+\frac{N}{N_{0}}\right)^{2 N_{0}}, & p=1
\end{array} .\right.
$$

It was shown by Fei et al. [75] that the potential cannot be expressed by $T$ in terms of elementary functions for arbitrary values of $p$. We therefore proceed with the case $p=1$. In this case

$$
N_{0}=\frac{N \delta_{n s}-1}{2-\delta_{n s}} \quad, \quad r=\frac{16 N_{0}}{N+N_{0}}=\frac{16\left(N \delta_{n s}-1\right)}{2 N-1} .
$$

Inserting $\delta_{n s}=0.032, N=60$ gives $N_{0}=0.5$ and $r=0.12$ which is too large in relation to the Planck data.

Inserting the expression of the potential for $p=1$ in Equation (6.33.124) into Equation (6.33.32) and integrating with $N\left(T_{0}\right)=0$ gives

$$
N= \begin{cases}N_{0}\left[1+\sqrt{\frac{V_{0}}{2}} \frac{1-2 N_{0}}{2 N_{0}}\left(T-T_{0}\right)\right]^{\frac{2}{1-2 N_{0}}}-N_{0}, & N_{0} \neq 1 / 2 \\ \frac{1}{2}\left[e^{\sqrt{2 V_{0}}\left(T-T_{0}\right)}-1\right] & ,\end{cases}
$$

Combining Equations (6.33.124) and (6.33.126) gives the potentials

$$
V(T)=\left\{\begin{array}{lll}
V_{0}\left[1+\sqrt{\frac{V_{0}}{2}} \frac{1-2 N_{0}}{2 N_{0}}\left(T-T_{0}\right)\right]^{\frac{4}{1-2 N_{9}}}, & N_{0} \neq 1 / 2 \\
V_{0} \exp \left[\sqrt{2 V_{0}}\left(T-T_{0}\right)\right] & , & N_{0} \neq 1 / 2
\end{array} .\right.
$$

Let us consider an inverse power law potential of the form

$$
V(T)=\frac{V_{0}}{1+\hat{T}^{p}} .
$$

Steer and Vernizzi [28] considered the special case with $p=4$. Inserting Equation (6.33.128) into Equation (6.33.20), the horizon slow roll parameters are found to be

$$
\varepsilon_{1}=\frac{p^{2} M_{P}^{2}}{2 V_{0}} \frac{\hat{T}^{2(p-1)}}{1+\hat{T}^{p}} \quad, \quad \varepsilon_{2}=\frac{p M_{P}^{2}}{V_{0}} \frac{\hat{T}^{p-2}\left[2(p-1)+(p-2) \hat{T}^{p}\right]}{1+\hat{T}^{p}} .
$$

Furthermore Steer and Vernizzi have assumed that $\hat{T}^{4}>>1$ and $V_{0} T_{0}^{2}>>M_{P}^{2}$. Hence we have to leading order

$$
\varepsilon_{1} \approx \frac{p^{2} M_{P}^{2}}{2 V_{0} T_{0}^{2}} \hat{T}^{p-2} \quad, \quad \varepsilon_{2} \approx \frac{p(p-2) M_{P}^{2}}{V_{0} T_{0}^{2}} \hat{T}^{p-2} .
$$

Inflation ends when $\varepsilon_{1}\left(\hat{T}_{f}\right)=1$ which gives

$$
\hat{T}_{f}^{p-2}=\frac{2 V_{0}}{p^{2} M_{p}^{2}} .
$$

It follows from Equations (6.33.35) and (6.33.128) that the number of e-folds is

$$
N(T)=\frac{V_{0} T_{0}^{2}}{p(p-2) M_{P}^{2}}\left(\frac{1}{T^{p-2}}-\frac{1}{T_{f}^{p-2}}\right) .
$$


The two last equations give

$$
\hat{T}^{2}=\frac{V_{0}^{2}}{M_{P}^{2}} \frac{1}{2(p-2) N+p^{2} / 2} .
$$

Inserting this into Equation (6.33.130) and using that $\varepsilon_{3}=-\left(\ln \varepsilon_{2}\right)_{N}$ gives

$$
\varepsilon_{1}=\frac{p}{2(p-2) N+p} \quad, \quad \varepsilon_{2}=\varepsilon_{3}=\frac{2(p-2)}{2(p-2) N+p} .
$$

Inserting these expressions into Equation (6.33.25) gives

$$
\delta_{n s}=\frac{4(p-1)}{2(p-2) N+p} \quad, \quad r=\frac{16 p}{2(p-2) N+p} \quad, \quad \alpha_{S}=-\frac{8(p-1)(p-2)}{[2(p-2) N+p]^{2}} .
$$

The case $p=4$ was considered by Steer and Vernizzi. It gives

$$
\delta_{n s}=\frac{3}{N+1} \quad, \quad r=\frac{16}{N+1} \quad, \quad \alpha_{S}=-\frac{3}{(N+1)^{2}} .
$$

Hence we get the relationships

$$
r=\frac{16}{3} \delta_{n s} \quad, \quad \alpha_{S}=-\frac{1}{3} \delta_{n s}^{2} .
$$

Inserting the Planck value $\delta_{n s}=0.032$ gives $r=0.17$ and $\alpha_{S}=-0.002$. Due to the large value of $r$ this model is ruled out by the Planck data.

It follows from the expressions (6.33.135) that

$$
r=\frac{4 p}{p-1} \delta_{n S} \quad, \quad \alpha_{S}=-\frac{p-2}{2(p-1)} \delta_{n s}^{2} .
$$

The first of these equations can be written

$$
p=\frac{r}{r-4 \delta_{n s}} .
$$

Inserting this into the second of the Equation (4.33.135) gives

$$
\alpha_{n s}=-\left(\delta_{n s}-\frac{r}{8}\right) \delta_{n s} .
$$

In order that $p$ shall be positive we must have $r>4 \delta_{n s}$. With $\delta_{n s}=0.032$ this gives $r>0.128$ while the Planck data favor $r<0.04$. Hence the Planck data favor negative values of $p$ because positive values give too large tensor-to-scalar ratio. Another way of seeing that the Planck data favor negative values of $p$ is to solve the first of the Equation (6.20.131) with respect to $p$. This gives

$$
p=\frac{4\left(N \delta_{n s}-1\right)}{(2 N+1) \delta_{n s}-4} .
$$

Inserting $N=60, \delta_{n s}=0.032$ gives $p=-29$. Substituting this into Equation (6.33.136) gives $r=0.123$. A smaller value of $r$ is favored, for example $r=0.05$. From Equation (6.33.139) we then get $p=-0.64$. Solving Equation (6.33.141) with respect to $N$ gives

$$
N=\frac{4(1-p)+p \delta_{n s}}{(2-p) 2 \delta_{n s}} .
$$


Putting $p=-0.64, \delta_{n s}=0.032$ into this equation gives $N=38$ which is too small. In Figure 13 we have plotted $p$ as given in Equation (6.20.137) as a function of $N$ for $\delta_{n s}=0.032$.

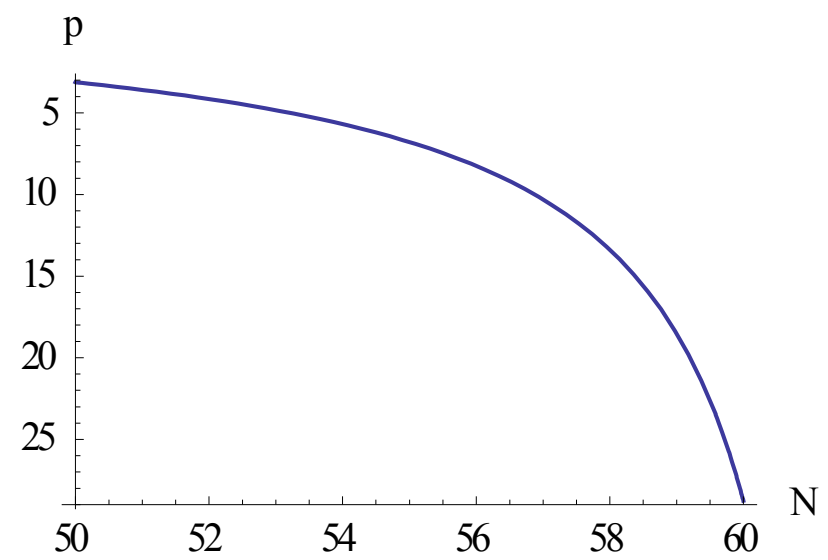

Figure 13. The parameter $p$ plotted as a function of $N$ as given in Equation (6.33.136) for $50<N<60$.

We see that the preferred values of $p$ are $-30<p<-3$.

Barbosa-Candejas et al. [201] considered a polynomial class of models with

$$
\varepsilon_{1}=\frac{\lambda}{N\left(N^{2 \lambda}+1\right)}
$$

Inserting the expression (6.33.143) into Equation (6.33.37) and performing the integration gives

$$
V(N)=\frac{V_{0}}{1+N^{-2 \lambda}} \quad, \quad V_{0}=V(0) .
$$

From Equations (6.33.143), (6.33.144) and (6.33.24) we get

$$
T(N)=\left\{\begin{array}{lc}
T_{1} N^{\frac{1-2 \lambda}{2}}, & \lambda \neq 1 / 2 \\
T_{1} \ln N & , \quad \lambda=1 / 2
\end{array},\right.
$$

where $T_{1}=\left[V_{0}(1-2 \lambda)^{2} / 8 \lambda M_{P}^{2}\right]^{2 \lambda /(2 \lambda-1)}$. Hence the potential as a function of the tachyon field is

$$
V(T)=\left\{\begin{array}{ll}
\frac{V_{0}}{1+\left(T / T_{1}\right)^{n}}, & \lambda \neq 1 / 2 \\
\frac{V_{0}}{1+\exp \left(-T / T_{1}\right)}, & \lambda=1 / 2
\end{array},\right.
$$

where $n=4 \lambda /(2 \lambda-1)$.

Inserting the expression (6.33.143) into Equation (6.33.28) we obtain the spectral parameters for this class of inflationary models

$$
\delta_{n s}=\frac{1+2 \lambda}{N}, \quad r=\frac{16 \lambda}{N\left(N^{2 \lambda}+1\right)} .
$$

This gives the $r, \delta_{n s}$-relationship

$$
r=\frac{8\left(N \delta_{n s}-1\right)}{N\left(N^{N \delta_{n s}-1}+1\right)}
$$

Inserting $\delta_{n s}=0.032,50<N<60$ gives $0.003<r<0.008$, which are acceptable values. 
Barbosa-Candejas et al. [201] also investigated an exponential class of models with

$$
\varepsilon_{1}=\frac{\lambda}{2\left(e^{\lambda N}+1\right)} .
$$

Inserting the expression (6.33.149) into Equation (6.33.37) and integrating gives

$$
V(N)=\frac{V_{0}}{1+\exp (-\lambda N)} .
$$

From Equations (6.33.149), (6.33.150) and (6.33.24) we obtain

$$
T(N)=T_{1} \exp (-\lambda N / 2) \quad, \quad T_{1}=2 M_{P} / \sqrt{\lambda V_{0}} .
$$

Hence the potential as a function of the inflaton field, is

$$
V(T)=\frac{V_{0}}{1+\left(T / T_{1}\right)^{2}} .
$$

Putting the expression (6.33.149) into Equation (6.33.28), we obtain the spectral parameters for this class of inflationary models

$$
\delta_{n s}=\lambda, \quad r=\frac{8 \lambda}{e^{\lambda N}+1} .
$$

Hence the $r, \delta_{n s}$-relationship is

$$
r=\frac{8 \delta_{n s}}{e^{N \delta}+1} .
$$

Inserting $\delta_{n s}=0.032,50<N<60$ gives $0.03<r<0.05$, which again are acceptable values.

Finally Barbosa-Candejas et al. [200] have studied the tachyonic version of S-dual inflation, with

$$
\varepsilon_{1}=\frac{\lambda}{\sinh (2 \lambda N)} .
$$

Inserting the expression (6.33.155) into Equation (6.33.37) and integrating leads to

$$
V(N)=V_{0} \tanh (\lambda N) .
$$

From Equations (6.33.155), (6.33.156), and (6.33.24) we obtain

$$
T(N)=\frac{\sqrt{\lambda}}{2} M_{P} \ln \tanh \left(\frac{\lambda N}{2}\right) .
$$

Hence the potential as a function of the inflaton field, is

$$
V(T)=\frac{V_{0}}{\cosh \left(\frac{\sqrt{\lambda V_{0}}}{M_{P}} T\right)} .
$$

Inserting the expression (6.33.155) into Equation (6.33.28) we obtain the spectral parameters for this class of inflationary models

$$
\delta_{n s}=2 \lambda \operatorname{coth}(\lambda N) \quad, \quad r=\frac{16 \lambda}{\sinh (2 \lambda N)} .
$$

These expressions can be combined to give

$$
\lambda=(1 / 4) \sqrt{\delta_{n s}\left(4 \delta_{n s}-r\right)} .
$$


Inserting $\delta_{n s}=0.032, r=0.04$ gives $\lambda=0.013$ in order to obtain agreement with the PBK-observations.

\subsubsection{Tachyon Warm Intermediate Brane Inflation}

V. Kamali, S. Basilakos and A. Mehrabi [204] have investigated tachyon warm-intermediate inflation in light of the Planck data. They have noted that while in cold standard tachyon inflation reheating is problematic because the tachyon fields in such models do not oscillate around the minimum of the potential, this problem can be alleviated in the context of warm inflation, where production of radiation occurs during the slow-roll era, which implies that reheating is not necessary.

For this class of models the density and pressure of the tachyon inflaton field is given by Equation (6.33.1). Inserting the expressions (6.33.1) into the brane version of the 1. Friedmann Equation (5.16.1), gives

$$
H^{2}=\frac{\kappa}{3} \frac{V}{\sqrt{1-\dot{T}^{2}}}\left(1+\frac{V}{2 \lambda \sqrt{1-\dot{T}^{2}}}\right) .
$$

The equations of continuity for the inflaton energy and the radiation energy are found in Equation (6.32.2). Inserting the expressions (6.33.1) into the equation of continuity of the tachyon field gives evolution equation of the tachyon field

$$
\frac{\ddot{T}}{1-\dot{T}^{2}}+3 H \dot{T}+\frac{V^{\prime}}{V}=-\frac{\Gamma}{V} \dot{T} \sqrt{1-\dot{T}^{2}} .
$$

Differentiating Equation (6.33.161), using that $\dot{V}=v^{\prime} \dot{\phi}$, and utilizing Equation (6.33.162) lead to

$$
\frac{6}{\mathcal{K}} H \dot{H}=-\dot{T}^{2}\left(1+\frac{V}{\lambda \sqrt{1-\dot{T}^{2}}}\right)\left(\Gamma+\frac{3 H V}{\sqrt{1-\dot{T}^{2}}}\right) .
$$

without any approximations. In the case of strong dissipation $\Gamma>>3 \mathrm{HV}$, and the equation reduces to

$$
\dot{T}^{2}=-\frac{6}{\kappa \Gamma} H \dot{H}\left(1+\frac{V}{\lambda \sqrt{1-\dot{T}^{2}}}\right)^{-1} .
$$

From Equation (6.33.161), we then get to lowest order in $V / \lambda$,

$$
\dot{T}^{2}=-(6 / \kappa \Gamma) H \dot{H}\left(1+3 H^{2} / \kappa \lambda\right)^{-1} .
$$

It may be noted that Equation (13) of Kamali, Basilakos and Mehrabi is slightly different. They have $\left(1+6 H^{2} / \kappa \lambda\right)^{-1 / 2}$ for the last factor, but a series expansion gives the expression (6.33.165) to lowest order in $H^{2} / \kappa \lambda$.

Kamali, Basilakos and Mehrabi have investigated two cases, I. $\Gamma=\Gamma_{0}=$ constant, and II. $\Gamma=\Gamma_{1} T_{R}$, where $T_{R}$ is the radiation temperature, and $\Gamma_{1}$ is a constant. With the number of e-folds $50<N<60$ they found for model I: $0.032<\delta_{n s}<0.037,0.004<r<0.009$, and for the model II: $0.031<\delta_{n s}<0.36,0.002<r<0.009$. Hence the models are in agreement with the Planck observational data.

\subsubsection{Tachyon Natural Inflation}

I shall here consider tachyon inflation with a potential like that of Equation (6.5.1)

$$
V=V_{0}(1+\cos \widetilde{T}), \quad \widetilde{T}=T / M .
$$


This is the same as the potential in Equation (2) of Rashidi and Nozari [205]. We shall here put their warp factor $\lambda=1$. With this potential Equations (6.33.15) and (6.33.23) give

$$
\varepsilon=\varepsilon_{H}=\frac{1}{2 \beta} \frac{1-\cos \widetilde{T}}{(1+\cos \widetilde{T})^{2}} \quad, \quad \eta=-\frac{1}{2 \beta} \frac{1}{1+\cos \widetilde{T}} \quad, \quad \eta_{H}=-\frac{1}{\beta} \frac{1}{(1+\cos \widetilde{T})^{2}}
$$

where $\beta=V_{0} \kappa^{2} M^{2}>0$. Note that Rashidi and Nozari have defined the slow roll parameters by the expressions (3.12) for $\varepsilon_{H}$ and $\eta_{H}$, (but without the factor 2 in the denominator in the expression for $\eta_{H}$, and calculated the second slow roll parameter by using the first expression in Equation (6.33.23) for $\eta$ instead of the second one for $\eta_{H}$.

The number of e-folds is found by inserting the potential (6.33.166) and its derivative into Equation (6.33.36), which gives

$$
N=\beta\left(\cos \widetilde{T}_{f}-\cos \widetilde{T}+2 \ln \frac{1-\cos \widetilde{T}_{f}}{1-\cos \widetilde{T}}\right) .
$$

The final value of the tachyon fiel is given by $\varepsilon\left(T_{f}\right)=1$ which leads to

$$
\cos \widetilde{T}_{f}=\frac{\sqrt{16 \beta+1}-4 \beta-1}{4 \beta} .
$$

The scalar spectral tilt, the tensor-toscalar-ratio and the running of the scalar spectral index are given by (4.13) and (4.4), respectively. Inserting the expressions (6.33.167) and introducing a function $-2<G(\beta)<0$ by

$$
G+1=\cos \widetilde{T}
$$

leads to

$$
\begin{gathered}
\delta_{n s}=-\frac{2}{\beta} \frac{G-1}{(G+2)^{2}}, \\
r=-\frac{8}{\beta} \frac{G}{(G+2)^{2}}, \\
\alpha_{S}=\frac{2}{\beta^{2}} \frac{(4-G) G}{(G+2)^{4}} .
\end{gathered}
$$

Solving Equation (6.33.171) with respect to $G$ gives

$$
G=-\frac{1+2 \beta \delta_{n s}-\sqrt{1+6 \beta \delta_{n s}}}{\beta \delta_{n s}} .
$$

where the minus sign has been chosen due to the condition $-2<G(\beta)<0$ which leads to the requirement $\beta>1 / 2 \delta_{n s}$. With $\delta_{n s}=0.032$ according to the Planck 2015 data, this demands that $\beta>15.6$.

Inserting the expression (6.33.174) into Equation (6.33.172) gives the $\left(r, \delta_{n s}\right)$-relationship

$$
r=\frac{1+2 \beta \delta_{n s}-\sqrt{1+6 \beta \delta_{n s}}}{\left(1-\sqrt{1+6 \beta \delta_{n s}}\right)} 8 \delta_{n s} .
$$

The tensor-to-scalar-ratio $r$ is plotted as a function of $\beta$ for $\delta_{n s}=0.032$ in Figure 14 . 


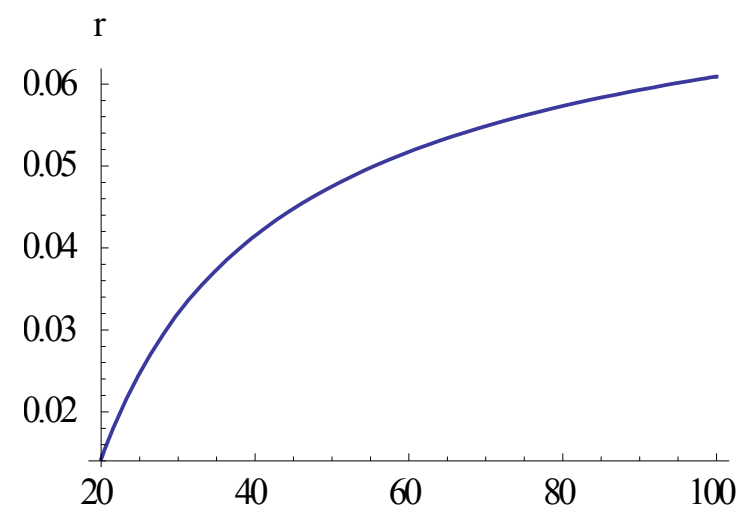

Figure 14. Tensor to scalar ratio as function of $\beta$.

Here $r$ is an increasing function of $\beta$ with $\lim _{\beta \rightarrow \infty} r=(8 / 3) \delta_{n s}$. Hence a prediction of this model is that $r<(8 / 3) \delta_{n s}$ or $r<0.085$ with $\delta_{n s}=0.032$.

\section{Conclusions}

The most recent analysis [46] (July 2017) of the combined Planck, BICEP2 and Keck results has given the restriction $r<0.04$ on the tensor-to-scalar-ratio for the CMB-radiation. This, together with the precise value of the scalar spectral index, $n_{s}=0.968 \pm 0.006$, determined from the observations, rule out several classes of inflationary universe models. The observations are still not sufficiently accurate to give precise values of the other spectral parameters. Hence we shall here focus mostly upon the predicted $\delta_{n s}, r-$ relationship of different inflationary models in order to judge how well they come out of the confrontation with observational data.

1. Polynomial inflation. The potential is given in Equation (6.1.1) and the $\delta_{n s}, r-$ relationship in (6.1.23). For $\delta_{n s}=0.032$ and $r<0.04$ this relationship requires $p<0.37$. Hence polynomial inflation with for example $p=2$ is ruled out by observations.

2. Hilltop inflation. The potential is given in Equation (6.2.1). It was noted below Equation (6.2.12) that small field hilltop inflation is ruled out by the observational data. As shown in Equation (6.2.30) large field hilltop inflation predicts $r<(8 / 3) \delta_{n s}=0.085$ which is in agreement with observations. However in general large field inflation has an unsecure theoretical foundation since the energy scale of the symmetry breaking is larger than the Planck energy. Strictly speaking we need a quantum gravity theory to describe such models.

3. Symmetry breaking inflation with potential (6.3.1). It was shown that this inflationary model predicts $4 \delta_{n s}<r<5 \delta_{n s}$, or with the Planck data, $0.128<r<0.16$. This is larger than the values, $r<0.04$, favored by the BICEP2/Planck-Keck data, so this model is ruled out.

4. Exponential potential inflation. For an inflation model with potential (6.4.1) the $\delta_{n s}, r-$ relationship is $r=8 \delta_{n s}=0.256$, which is in conflict with observations. However it is possible for models with the more general form (6.4.13) of the potential to be in agreement with observations.

5. Natural inflation. The potentials are given in Equation (6.5.1). It was shown from the $\delta_{n s}$, $r$ - relationship (6.5.24) that the symmetry breaking mass is $M=7 M_{P}$, i.e., much larger than the Planck mass in these models, which is somewhat problematic, since we are then outside the region of validity of the classical theory of relativity.

6. Hybrid natural inflation with potential (6.6.1). It was shown that the hybrid natural inflation models are in trouble unless $\alpha_{S}>0$ and $r<4 \alpha_{s}$.

7. Higgs or Starobinsky inflation. The simplest form of the potential is given in Equation (6.7.2). This model predicts that $r=3 \delta_{n s}^{2}=0.003$ which is in agreement with observations. The more general potential (6.7.13) gives the $\delta_{n s}, r$ - relationship $r \approx\left(2 / q^{2}\right) \delta_{n s}^{2}$. In general the 
Higgs-Starobinsky inflation models predict very small values of $r$. It should be noted, however, that there may exist a limit to how small $r$ is allowed to be. Hamada and coworkers [206] have recently argued in the context of Higgs inflation, that the PandaX-II bound on the dark-matter mass, $m_{D M}>750 \mathrm{GeV}$, leads to the requirement $r>10^{-3}$ in most of the parameter space of elementary particle physics.

8. S-dual inflation. These models have in general a potential of the form (6.8.2). It was shown that these models do not have a graceful exit of the slow roll era. Also they have an extra free parameter making exact predictions problematic.

9. Hyperbolic inflation. The potential is given in Equation (6.9.1). These models are similar to those of the S-dual inflation, but they do not suffer from the exit problem. With suitable values of the two free parameters of these models they give values of the spectral parameters in accordance with observations.

10. M-flation. The potential is given in Equation (6.10.1). This model predicts too large tensor-to-scalar ration and is ruled out by observations.

11. Supergravity motivated inflation. These models have the potential (6.11.1) with two arbitrary parameters, that can be chosen so that a model in this class is in agreement with the observational data. There is also a so-called $\alpha$ - attractor model with a potential (6.11.9). This is in agreement with observations for $\alpha<25$.

12. Goldstone inflation with potential (6.12.1). This model is mathematically identical to one of the natural inflation models and is a large field model. It can be adjusted to be in accordance with observations, but has the same foundational problems as natural inflation.

13. Coleman-Weinberg inflation with potential (6.13.1). This class of models incorporates both small-fields and large-field models. The small field version is in agreement with observations, is physically well motivated, and is a promising inflation model.

14. Kähler moduli inflation with potential (6.14.1). This model predicts a very small value of $\mathrm{r}$ and is in agreement with the present observational data.

15. Hybrid inflation. Inflation models in this class have two fields, a so-called water-fall field and an inflaton field. The simplest version with potential (6.15.1) predicts too large value of $r$ and is thus ruled out by the observational data.

16. Brane inflation. The predicted tensor-to-scalar ratio for brane inflation with the polynomial potential (6.1.1) is given in Equation (6.16.29), which leads to the value $r_{B}=0.096$. According to the most recent analysis of the observational data $r<0.04$, so this brane model is ruled out by observations.

17. Fast roll inflation with potential (6.17.1). This model predict $r=8 \delta_{n s}=0.256$. Hence it is ruled out by the observational data due to the high value it predicts for $r$.

18. Running mass inflation with potential (16.18.1). This model has three free parameters, and hence it cannot predict the values of the optical parameters.

19. K-inflation. Like fast roll inflation this class of models predict $r=8 \delta_{n s}=0.256$ and is thus ruled out by the observational data.

20. Dirac-Born-Infield inflation. A class of DBI-inflationary models with polynomial potential $V \propto \phi^{p}$ has been considered. According to Equation (6.20.2), it predicts $r=0.096$, and is hence in conflict with observational data.

21. Flux-brane inflation with potential (6.21.1). This model predicts $\delta_{n s} \approx 1 / N$ giving $N \approx 31$ for $\delta_{n s}=0.032$. This is lower than admitted in order to solve the horizon- and flatness problems.

22. Mutated hilltop inflation with potential (6.22.1). This model is not ruled out by the Planck/BICEP2 observations.

23. Arctan inflation with potential (6.23.1). In this model $\delta_{n s} \simeq 4 / 3 N$ showing that the number of e-folds during the slow roll era is $N=42$, which is a little less that the optimal number for solving the horizon and flatness problems. 
24. Inflation with fractional potential. One version of this model has potential (6.24.1). This leads to the prediction that for $N<60$ the tensor-to-scalar ratio is $r<0.02$. Another version has potential (6.24.8) having a too small number of e-folds during the slow roll era to give a realistic inflationary scenario. A third version has potential (6.24.14) giving $N \approx 47$, which is close to being acceptable.

25. Twisted inflation with potential (6.25.1). The tensor-to-scalar ratio has a very small value according to the twisted inflation model. It seems to be an acceptable inflation model.

26. Inflation with invariant density spectrum. This model has potential (6.26.1) and has a scale invariant Harrison-Zeldovich density fluctuation spectrum. Also, the number of e-folds is less than one. Hence, this model is ruled out as a realistic inflationary model.

27. Quintessential inflation. A first version has potential (6.27.6). This model of quintessential inflation predicts $r>0.128$ and is thus ruled out by observations. A second version has potential (6.27.8). Using $\delta_{n s}=0.032$ it predicts $N \approx 62$ and $r \simeq 0$. This model is in agreement with observations. A third model has potential (6.27.14). With $\delta_{n s}=0.032$ this model gives $N>62.5$ and $r \approx 4 \delta_{n s}^{2}=0.004$ in agreement with observations. A fourth model has potential (6.27.26) and turned out to be very unrealistic.

28. Generalized Chaplygin gas inflation. The inflaton field has a potential given in Equation (6.28.13). With $\delta_{n s}=0.032$ this model predicts $N=168$. Since the number of e-folds is usually restricted to $50<N<60$ it is concluded that a universe dominated by generalized Chaplygin gas is not a suitable model of the inflationary era.

29. Axion monodromy inflation with potential (6.29.1). With a reasonable value of a parameter in this model one obtains $r<0.04$ in agreement with the observational data.

30. Intermediate inflation. The potential is given in Equation (6.30.8). The simplest versions of these models predict that $r>8 \delta_{n s}=0.256$, which is not allowed by observations.

31. Constant rate of roll inflation. Maybe the most promising version of this class of models is the one with potential (6.31.45). However, the potential contains two arbitrary parameters, and this prevents a prediction of the tensor-to-scalar-ratio unless one can determine for example an initial condition defining the beginning of the slow roll era. Recently Yi and Gong [207] has shown that the model with potential (6.31.25) and $\beta<0$ is in conflict with the Planck data.

32. Warm inflation. During the evolution of warm inflation dissipative effects are important, and inflaton field energy is transformed to radiation energy. This is a large class on inflationary models that may be realized in a large number of ways. In the warm inflation scenario a thermalized radiation component is present with temperature $T>H$, where both $T$ and $H$ are expressed in units of energy. Then the tensor-to-scalar ratio is suppressed by the factor $(T / H)(1+Q)^{5 / 2}$ when compared with the standard cold inflation, where $Q$ the so-called dissipative ratio defined in Equation (6.32.5).

Let us summarize the predictions of some specific models.

Warm polynomial inflation with an inflaton potential given by Equation (6.1.1) with $p=4$ has been investigated by Panotopoulos and Videla [182]. They found that in the weak dissipative regime when $Q<<1$ the scalar spectral tilt is $\delta_{n s}=1 / N$, giving $N=31$ which is too small to be compatible with the standard inflationary scenario. However, in the strong dissipative regime when $Q>>1$, the spectral parameters can be made to be in accordance with the observational data by choosing a proper value of an arbitrary parameter.

Taylor and Berera [195] have briefly considered warm inflation models with an exponential potential, $V=V_{0} \exp (\phi / M)$, and found that in the strong dissipation regime the scalar spectral index parameter $\delta_{n s}$ for models of this type is negative in conflict with the Planck observations.

Visinelli [189] has investigated warm natural inflation with potential (6.32.48). He found that this class of inflationary models predicts a vanishing value of the tensor-to-scalar-ratio. 
Several versions of warm viscous inflation have also been investigated, and their properties have been restricted in order to obtain agreement with the observational data. In all such models, the tensor-to-scalar-ratio has a small value, but without some fundamental theory making it possible to determine some physical parameters they cannot presdict the values of the CMB spectral parameters.

33. Tachyon inflation. Tachyon inflation is a string theory inspired model of inflation. In these models it has become usual to introduce a so-called tachyon field, and denote it by $T$. The tachyon field is related to the usual inflaton field by Equation (6.33.16). Like warm inflation, this is a large class of inflationary models that may be realized in a large number of ways. Here, too, we shall summarize the predictions of some specific models.

A class of models with $\varepsilon_{1}=\beta / N$, where $\beta$ is an arbitrary constant, leads to the relationship $r=8\left(\delta_{n s}-1 / N\right)$. With $N=50, \delta_{n s}=0.032$ we get $\beta=0.3, r=0.096$. This value of the tensor-to-scalar ratio is larger than allowed by the most recent analysis of the observational data [46], $r<0.04$. This class of models have members with polynomial or exponential tachyon potentials.

As shown in Figure 12, tachyon models with $\delta_{n s}=p /(N+A)$ have $r<0.04$ when $0<A<0.29$. Several other tachyon models may be made to agree with observations, but having more than one free parameter, that is not a prediction of the models, only adjustments after the observational results have been obtained.

It has been shown that tachyon natural inflation predicts $r<(8 / 3) \delta_{n s}$ or $r<0.085$ with $\delta_{n s}=0.032$.

It should also be mentioned that S. Chervon and coworkers [208-210] have developed a procedure for calculating the optical parameters of inflationary universe models exactly, without applying the slow roll approximation. One may show that the predictions made by means of such calculations deviate only a few per cent from those based upon the slow roll approximations.

In the present article I have given a systematic exposition of the dynamics of inflationary models, the three types of slow roll parameters-the potential-, the Hubble-, and the horizon-flow parameters-, and the $N$-formalism of inflationary models. Furthermore 33 classes of inflationary models have been described, many of them in a rather detailed way. Their predictions of the tensor-to-scalar-ratio has been calculated, given the measured scalar tilt, $\delta_{n s}=0.032$, and the proper range of the number of e-folds, $50<N<60$, and compared to the requirement $r<0.04$ of the most recent analysis of the observational data. A supplementary review has been given in [211].

The main result is that many inflationary models can be ruled out because they predict to large value of $r$, and a few are strongly favored. Models that are ruled out by the observational data are: Most types of polynomial inflation, small field hilltop inflation, the main type of symmetry breaking inflation, the simples types of exponential potential inflation, some types of hybrid natural inflation, M-flation, the simplest types of hybrid inflation, brane inflation, fast roll inflation, K-inflation, Arctan-inflation, inflation with invariant density spectrum, two of the four models of quintessential inflation that were reviewed, generalized Chaplygin gas inflation, the simplest versions of intermediate inflation, and some types of tachyon inflation.

Other models are not attractive because they contain too may arbitrary parameters, implying that they have a rather phenomenological character and lack predictive force, or that their theoretical foundations are weak, such as for large field inflations that strictly speaking need a quantum gravity theory because their symmetry breaking energy is larger than the Planck energy, or they have no graceful exit of the slow roll era. Models in this category are: large field hilltop inflation, natural inflation, S-dual inflation, hyperbolic inflation, supergravity motivated inflation, Goldstone inflation, large field Coleman-Weinberg inflation and constant rate of roll inflation. 
The models of inflation that come best out of the confrontation with observational data, have a graceful exit of the slow roll era, and are also able to predict a value for $r$ given the restrictions mentioned above of $\delta_{n s}$ and $N$ are: Higgs or Starobinsky inflation, small field Coleman-Weinberg inflation, Kähler moduli inflation, standard DBI-inflation, mutated hilltop inflation, some versions of inflation with fractional potential, twisted inflation, some types of quintessential inflation, axion monodromy inflation, and many types of warm inflation.

In general, warm inflation seems to be the class with physically most realistic inflationary models, and also those that come best out of the confrontation with observational data.

Conflicts of Interest: The author declares no conflicts of interest.

\section{References}

1. Ade, P.; Aghanim, N.; Armitage-Caplan, C.; Arnaud, M.; Ashdown, M.; Atrio-Barandela, F.; Aumont, J.; Baccigalupi, C.; Banday, A.J.; Barreiro, R.B.; et al. Planck 2013 results. XXX. Cosmic infrared background measurements and implications for star formation. Astron. Astrophys. 2014, 571, A30.

2. Ade, P.; Aghanim, N.; Ahmed, Z.; Aikin, R.W.; Alexander, K.D.; Arnaud, M.; Aumont, J.; Baccigalupi, C.; Banday, A.J.; Barkats, D.; et al. Joint Analysis of BICEP2/Keck Array and Planck Data. Phys. Rev. Lett. 2015, 114, 101301. [CrossRef] [PubMed]

3. Watson, G.S. An Exposition on Inflationary Cosmology. arXiv 2000, arXiv:astro-ph/0005003.

4. Linde, A.D. Inflationary Cosmology. Lect. Notes Phys. 2008, 738, 1-54.

5. Kinney, W.H. Cosmology, inflation, and the physics of nothing. In Techniques and Concepts of High-Energy Physics XII; NATO Science Series; Springer: Berlin, Germany, 2003; Volume 123, pp. 189-243.

6. Ade, P.; Aghanim, N.; Armitage-Caplan, C.; Arnaud, M.; Ashdown, M.; Atrio-Barandela, F.; Aumont, J.; Baccigalupi, C.; Banday, A.J.; Barreiro, R.B.; et al. Planck 2013 results. XVI. Cosmological parameters. Astron. Astrophys. 2014, 571, A16.

7. Ade, P.; Aghanim, N.; Armitage-Caplan, C.; Arnaud, M.; Ashdown, M.; Atrio-Barandela, F.; Aumont, J.; Baccigalupi, C.; Banday, A.J.; Barreiro, R.B.; et al. Planck 2013 results. XXII. Constraints on inflation. Astron. Astrophys. 2014, 571, A22.

8. Germán, G.; Herrera-Aguilar, A.; Hidalgo, J.C.; Sussman, R.A. Canonical single field slow-roll inflation with a non-monotonic tensor-to-scalar ratio. J. Cosmol. Astropart. Phys. 2016, 2016, 025. [CrossRef]

9. Liddle, A.R.; Parsons, P.; Barrow, J.D. Formalizing the slow roll approximation in inflation. Phys. Rev. D 1994, 50, 7222-7232. [CrossRef]

10. Peiris, H.V.; Komatsu, E.; Verde, L.; Spergel, D.N.; Bennett, C.L.; Halpern, M.; Hinshaw, G.; Jarosik, N.; Kogut, A.; Limon, M.; et al. First-year Microwave Anisotropy Probe (WMAP) Observations: Implications for Inflation. Astrophys. J. Suppl. 2003, 148, 213-231. [CrossRef]

11. Schwarz, D.J.; Terrero-Escalante, C.A. Primordial fluctuations and cosmological inflation after WMAP1. J. Cosmol. Astropart. Phys. 2004, 2004, 003. [CrossRef]

12. Creminelli, P.; Nacir, D.L.; Simonović, M.; Trevisan, G.; Zaldarriaga, M. $\Phi^{2}$ Inflation at its Endpoint. Phys. Rev. D 2014, 90, 083513. [CrossRef]

13. Correia, F.P.; Schmidt, M.G.; Tavartkiladze, Z. Natural Inflation from 5D SUGRA and Low Reheat Temperature. Nucl. Phys. B 2015, 898, 173-196. [CrossRef]

14. Kinney, W.H. Inflation: Flow, fixed points and observables to arbitrary order in slow roll. Phys. Rev. D 2002, 66, 083508. [CrossRef]

15. Kolb, E.W. A coasting cosmology. Astrophys. J. 1989, 344, 543-550. [CrossRef]

16. Turok, N. Global Texture as the Origin of Cosmic Structure. Phys. Rev. Lett. 1989, 63, 2625-2628. [CrossRef] [PubMed]

17. Grøn, Ø.; Johannesen, S. Conformally flat spherically symmetric spacetimes. Eur. Phys. J. Plus 2013, 128, 92. [CrossRef]

18. Leach, S.M.; Liddle, A.R.; Martin, J.; Schwarz, D.J. Cosmological parameter estimation and the inflationary cosmology. Phys. Rev. D 2002, 66, 023515. [CrossRef] 
19. Martin, J. The Observational Status of Cosmic Inflation After Planck. In The Cosmic Microwave Background; Fabris, J., Piattella, O., Rodrigues, D., Velten, H., Zimdahl, W., Eds.; Astrophysics and Space Science Proceedings; Springer: Cham, Switzerland, 2016; Volume 45.

20. Spaliński, M. New Solutions of the Inflationary flow equations. J. Cosmol. Astropart. Phys. 2007, $2007,016$. [CrossRef]

21. Lyth, D.H. What would we learn by detecting a gravitational wave signal in the cosmic microwave background anisotropy? Phys. Rev. Lett. 1997, 78, 1861-1863. [CrossRef]

22. Lyth, D.H.; Riotto, A. Particle Physics Models of Inflation and Cosmological Density Perturbation. Phys. Rep. 1999, 314, 1-146. [CrossRef]

23. Easther, R.; Kinney, W.H.; Powell, B.A. The Lyth Bound and the End of Inflation. J. Cosmol. Astropart. Phys. 2006, 2006, 004. [CrossRef]

24. Germán, G. On the Lyth bound and single-field slow roll inflation. arXiv 2014, arXiv:1405.3246.

25. Coone, D.; Roest, D.; Vennin, V. The Hubble flow of Plateau Inflation. J. Cosmol. Astropart. Phys. 2015, 2015, 010. [CrossRef]

26. Vennin, V. Horizon-Flow off-track for Inflation. Phys. Rev. D 2014, 89, 083526. [CrossRef]

27. Martin, J.; Ringeval, C.; Vennin, V. Encyclopædia Inflationaris. Phys. Dark Univ. 2014, 5-6, 75-235. [CrossRef]

28. Steer, D.A.; Vernizzi, F. Tachyon inflation: Tests and comparison with single scalar field inflation. Phys. Rev. D 2004, 70, 043527. [CrossRef]

29. Myrzakulov, R.; Sebastiani, L.; Zerbini, S. Reconstruction of Inflation Models. Eur. Phys. J. C 2015, 75, 215. [CrossRef]

30. Mukhanov, V. Quantum Cosmological Perturbations: Predictions and Observations. Eur. Phys. J. C 2013, 73, 2486. [CrossRef]

31. Ballesteros, G.; Casas, J.A. Large tensor-to-scalar ratio and running of the scalar spectral index with instep inflation. Phys. Rev. D 2015, 91, 043502. [CrossRef]

32. Kinney, W.H. Horizon crossing and inflation with large $\eta$. Phys. Rev. D 2005, 72, 023515. [CrossRef]

33. Martin, J.; Motohashi, H.; Suyama, T. Ultra Slow-Roll Inflation and the non-Gaussianity Consistency Relation. Phys. Rev. D 2013, 87, 023514. [CrossRef]

34. Dimopoulos, K. Slow-roll versus ultra slow-roll inflation. Phys. Lett. B 2017, 775, 262-265. [CrossRef]

35. Ade, P. Planck 2015 results. XX. Cosmological parameters. Astron. Astrophys. 2016, 594, A13.

36. Ade, P. Planck 2015 results. XIII. Constraints on inflation. Astron. Astrophys. 2016, 594, A20.

37. Huang, Q. Lyth bound revisited. Phys. Rev. D 2015, 91, 123532. [CrossRef]

38. Baumann, D. TASI Lectures on Inflation. arXiv 2012, arXiv:0907.5424.

39. Ade, P.; Aikin, R.W.; Barkats, D.; Benton, S.J.; Bischoff, C.A.; Bock, J.J.; Brevik, J.A.; Buder, I.; Bullock, E.; Dowell, C.D.; et al. BICEP 2 I: Detection of B-mode Polarization at Degree Angular scales. Phys. Rev. Lett. 2014, 112, 241101. [CrossRef] [PubMed]

40. Ashoorioon, A.; Dimopoulos, K.; Sheikh-Jabbari, M.M.; Shiu, G. Non-Bunch-Davis Initial State Reconciles Chaotic Models with BICEP and Planck. Phys. Lett. B 2014, 737, 98-102. [CrossRef]

41. Gao, Q.; Gong, Y.; Li, T. The Modified Lyth Bound and Implications of BICEP2 Results. Phys. Rev. D 2015, 92, 063509. [CrossRef]

42. Gao, Q.; Gong, Y. The challenge for the single field inflation with BICEP2 result. Phys. Lett. B 2014, 734, 41-43. [CrossRef]

43. Gao, X.; Li, T.; Shukla, P. Fractional-chaotic inflation in the lights of PLANCK and BICEP2. Phys. Lett. B 2014, 738, 412-417. [CrossRef]

44. Benetti, M.; Ramos, R.O. Warm dissipative effects predictions and constraints from the Planck data. Phys. Rev. D 2017, 95, 023517. [CrossRef]

45. Bamba, K.; Odintsov, S.; Saridakis, E.N. Inflationary Cosmology in unimodular F(T) gravity. Mod. Phys. Lett. A 2017, 32, 1750114. [CrossRef]

46. Gott III, J.R.; Colley, W.N. Reanalysis of the BICEP2, Keck and Planck Data: No Evidence for Gravitational Radiation. arXiv 2017, arXiv:1707.06755.

47. Minor, K.E.; Kaplinghat, M. Inflation that runs naturally, gravitational waves and suppression of power at large and small scales. Phys. Rev. D 2014, 91, 063504. [CrossRef]

48. Antusch, S.; Nolde, D. BICEP2 implications for single field slow-roll inflation revisited. J. Cosmol. Astropart. Phys. 2014, 2014, 035. [CrossRef] 
49. Bramante, J.; Lehman, L.; Martin, A. Clearing the Brush: The Last Stand of Solo Small Field Inflation. Phys. Rev. D 2014, 90, 023530. [CrossRef]

50. Barenboim, G.; Park, Wan-Il. On the tensor-to-scalar ratio in large single-field inflation models. arXiv 2015, arXiv:1509.07132.

51. Huang, Q.; Wang, S. No evidence for the blue-tilted power spectrum of relic gravitational waves. J. Cosmol. Astropart. Phys. 2015, 2015, 021. [CrossRef]

52. Stewart, E.D.; Lyth, D.H. A more accurate analytic calculation of the spectrum of cosmological perturbations produced during inflation. Phys. Lett. B 1993, 302, 171-175. [CrossRef]

53. Huang, Q. Slow roll reconstruction for running spectral index. Phys. Rev. D 2007, 76, 043505. [CrossRef]

54. Vallinotto, A.; Copeland, E.J.; Kolb, E.W.; Liddle, A.R.; Steer, D.A. Inflationary potentials yelding constant scalar perturbation spectral indices. Phys. Rev. D 2004, 69, 103519. [CrossRef]

55. Hodges, H.; Blumenthal, G. Arbitrariness of inflationary fluctuation spectra. Phys. Rev. D 1990, 42, 3329. [CrossRef]

56. Amoros, J.; de Haro, J. The twilight of the single slow rolling inflation. arXiv 2015, arXiv:1503.02153.

57. Czerny, M.; Kobayashy, T.; Takahashi, F. Running Spectral Index from Large-field Inflation with modulations Revisited. Phys. Lett. B 2014, 735, 176-180. [CrossRef]

58. Carrillo-González, M.; German, G.; Herrera-Aguilar, A.; Hidalgo, J.C.; Sussman, R. Testing hybrid natural inflation with BICEP2. Phys. Lett. B 2014, 734, 345-349. [CrossRef]

59. Cheng, C.; Huang, Q.G. The Tilt of Primordial Gravitational WavesSpectra from BICEP2. Mod. Phys. Lett. A 2014, 29, 1450185.

60. Cheng, C.; Huang, Q.G. Constraints on the cosmological parameters from BICEP2, Planck and WMAP. Eur. Phys. J. C 2014, 74, 3139. [CrossRef]

61. Barbosa-Cendejas, N.; De-Santiagoa, J.; Germana, G.; Hidalgoa, J.C.; Mora-Lunaa, R.R. Tachyon inflation in the N-formalism. J. Cosmol. Astropart. Phys. 2015, 2015, 020. [CrossRef]

62. Engqvist, K.; Mazumdar, A.; Stephens, P. Inflection point inflation within supersymmetry. J. Cosmol. Astropart. Phys. 2010, 2010, 020. [CrossRef]

63. Choi, S.-M.; Lee, H.M. Inflection point inflation and reheating. Eur. Phys. J. C 2016, 76, 303. [CrossRef]

64. Okada, N.; Okada, S.; Raut, D. Inflection-point inflation in hyper-charge oriented U(1)X model. Phys. Rev. D 2017, 95, 055030. [CrossRef]

65. Bamba, K.; Nojiri, S.; Odintsov, S.D. Reconstruction of scalar field theories realizing inflation consistent with the Planck and BICEP2 results. Phys. Lett. B 2014, 737, 374-378. [CrossRef]

66. Garcia-Bellido, J.; Roest, D. The large-N running of the spectral index of inflation. Phys. Rev. D 2014, 89, 103527. [CrossRef]

67. Garcia-Bellido, J.; Roest, D.; Scalisi, M.; Zavala, I. The Lyth Bound of Inflation with a Tilt. Phys. Rev. D 2014, 90, 123539. [CrossRef]

68. Bamba, K.; Odintsov, S.D. Inflation in a viscous fluid model. Eur. Phys. J. C 2016, 76, 18. [CrossRef]

69. Chiba, T. Reconstructing the inflaton potential from the spectral index. Progr. Theor. Exp. Phys. 2015, 2015, 073E02. [CrossRef]

70. Roest, D. Universality classes of inflation. J. Cosmol. Astropart. Phys. 2014, 2014, 007. [CrossRef]

71. Mukhanov, V. Inflation without selfreproduction. Prog. Phys. 2014, 63, 36-41. [CrossRef]

72. Lin, J.; Gao, Q.; Gong, Y. The reconstruction of inflationary potentials. Mon. Not. R. Astron. Soc. 2016, 459, 4029-4037. [CrossRef]

73. Barranko, L.; Boubekeur, L.; Mena, O. A model-independent fit to Planck and BICEP2 data. Phys. Rev. D 2014, 90, 063007. [CrossRef]

74. Gao, Q.; Gong, Y. Reconstruction of extended inflationary potentials for attractors. arXiv 2017, arXiv:1703.02220.

75. Fei, Q.; Gong, Y.; Lin, J.; Yi, Z. The reconstruction of Tachyon inflationary potentials. J. Cosmol. Astropart. Phys. 2017, 2017, 018. [CrossRef]

76. Creminelli, P.; Dubovsky, S.; Nacir, D.L.; Simonović, M.; Trevisan, G.; Villadoro, G.; Zaldarriaga, M. Implications of the scalar tilt for the tensor-to-scalar ratio. Phys. Rev. D 2015, 92, 123528. [CrossRef]

77. Gobetti, R.; Pajer, E.; Roest, D. On the Three Primordial Numbers. J. Cosmol. Astropart. Phys. 2015, $2015,058$. [CrossRef] 
78. Koh, S.; Lee, B.H.; Tumurtushaa, G. Reconstruction of the Scalar Field Potential in Inflationary Models with a Gauss-Bonnet term. Phys. Rev. D 2017, 95, 123509. [CrossRef]

79. Kallosh, R.; Linde, A. Universality Class in Conformal Inflation. J. Cosmol. Astropart. Phys. 2013, $2013,002$. [CrossRef]

80. Davis, J.L.; Levi, T.S.; Van Raamsdonk, M.; Whyte, K.R.L. Twisted Inflation. J. Cosmol. Astropart. Phys. 2010, 2010, 032. [CrossRef]

81. Biagetti, M.; Kehagias, A.; Riotto, A. What We Can Learn from the Running of the Spectral Index if no Tensors are Detected in the Cosmic Microwave Background Anisotropy. Phys. Rev. D 2015, 91, 103527. [CrossRef]

82. Binétruy, P.; Kiritsis, E.; Mabillard, J.; Pieroni, M.; Rosset, C. Universality classes for models of inflation. J. Cosmol. Astropart. Phys. 2015, 2015, 033. [CrossRef]

83. Linde, A.D. Inflationary Cosmology after Planck 2013. arXiv 2014, arXiv:1402.0526.

84. Linde, A.D. Chaotic Inflation. Phys. Lett. B 1983, 129, 177-181. [CrossRef]

85. Clesse, S. An introduction to inflation after Planck: From theory to observations. arXiv 2015, arXiv:1501.00460.

86. Barrow, J.D.; Liddle, J.D. Perturbation spectra from intermediate inflation. Phys. Rev. D 1993, 47, R5219-R5223. [CrossRef]

87. Rezazadeh, K.; Karami, K.; Hashemi, S. Tachyon inflation with steep potentials. Phys. Rev. D 2017, 95, 103506. [CrossRef]

88. Kinney, W.H.; Kolb, E.W.; Melchiorri, A.; Riotto, A. Inflation model constraints from the Wilkinson Anisotropy Probe three-year data. Phys. Rev. D 2006, 74, 023502. [CrossRef]

89. Chiba, T.; Kohri, K. Consistency Relations for Large Field Inflation. Prog. Theor. Exp. Phys. 2014, $2014,093 \mathrm{E} 01$. [CrossRef]

90. Kobayashi, T.; Seto, O. Polynomial inflation models after BICEP2. Phys. Rev. D 2014, 89, 103524. [CrossRef]

91. Destri, C.; de Vega, H.J.; Sanchez, N.G. MCMC analysis of WMAP3 and SDSS data points to broken symmetry inflation potentials and provides a lower bound on the tensor to scalar ratio. Phys. Rev. D 2008, 77, 043509. [CrossRef]

92. de Haro, J.; Amorós, J.; Pan, S. Simple inflationary quintessential model. Phys. Rev. D 2016, 93, 084018. [CrossRef]

93. Boubekeur, L.; Lyth, D.H. Hilltop Inflation. J. Cosmol. Astropart. Phys. 2005, 2005, 010. [CrossRef]

94. Kohri, K.; Lin, C.M.; Lyth, D.H. More hilltop inflation models. J. Cosmol. Astropart. Phys. 2007, $2007,004$. [CrossRef]

95. Basilakos, S.; Lima, J.A.S.; Solà, J. A viable Starobinsky-like inflationary scenario in the light of Planck and BICEP2 results. Int. J. Mod. Phys. D 2014, 23, 1442011. [CrossRef]

96. Kinney, W.H.; Mahanthappa, K.T. Inflation at Low Scales: General Analysis and a Detailed Model. Phys. Rev. D 1996, 53, 5455-5467. [CrossRef]

97. Zarei, M. On the running of the spectral index to all orders: A new approach to constraint the inflationary models. Class. Quantum Gravity 2016, 33, 115008. [CrossRef]

98. Lyth, D.H.; Liddle, A.R. The Primordial Density Perturbation; Cambridge University Press: Cambridge, UK, 2009.

99. Pieroni, M. Classification of Inflationary Models and Constraints on Fundamental Physics. Ph.D. Thesis, Paris Diderot University, Paris, France, 2016.

100. Shiu, G.; Henry Tye, S.H. Some aspects of brane inflation. Phys. Lett. B 2001, 516, 421-430. [CrossRef]

101. Drees, M.; Erfani, E. Running Spectral Index and Formation of Primordial Black Hole in Single Field Inflation Models. J. Cosmol. Astropart. Phys. 2012, 2012, 035. [CrossRef]

102. Lu, Z. Inflation in the Generalized Inverse Power Law Scenario. J. Cosmol. Astropart. Phys. 2013, $2013,038$. [CrossRef]

103. Chung, Y.; Lin, E. Topological inflation with large tensor-to-scalar-ratio. J. Cosmol. Astropart. Phys. 2014, 2014, 020. [CrossRef]

104. Escudero, M.; Ramírez, H.; Boubekeur, L.; Giusarma, E.; Mena, O. The present and future of the most favored inflationary models after Planck 2015. J. Cosmol. Astropart. Phys. 2016, 2016, 020. [CrossRef]

105. Rehman, M.U.; Shafi, Q.; Wickman, J.R. GUT Inflation and Proton Decay after WMAP 5. Phys. Rev. D 2008, 78, 123516. [CrossRef]

106. Liddle, R.A. Power-law inflation with exponential potentials. Phys. Lett. B 1989, 4, 502-508. [CrossRef] 
107. Motohashi, H.; Starobinsky, A.A.; Yokoyama, J. Inflation with a constant rate of roll. J. Cosmol. Astropart. Phys. 2015, 2015, 018. [CrossRef]

108. Lucchin, F.; Matarrese, S. Power-law Inflation. Phys. Rev. D 1985, 32, 1316. [CrossRef]

109. Story, K.T.; Reichardt, C.L.; Hou, Z.; Keisler, R.; Aird, K.A.; Benson, B.A.; Bleem, L.E.; Carlstrom, J.E.; Chang, C.L.; Cho, H.; et al. A measurement of the cosmic microwave background damping tail from the 2500-square-degree SPT-Sz survey. Astrophys. J. 2013, 779, 86. [CrossRef]

110. Unnikrishnan, U.; Sahni, V. Resurrecting power law inflation in the light of Planck results. J. Cosmol. Astropart. Phys. 2013, 2013, 063. [CrossRef]

111. Geng, C.Q.; Hossain, W.; Myrzakulov, R.; Sami, M.; Saridakis, E.N. Quintessential inflation with canonical and noncanonical scalar fields and Planck 2015 results. Phys. Rev. D 2015, 92, 023522. [CrossRef]

112. Alcaniz, J.S.; Carvalho, F.C. $\beta$-exponential inflation. Europhys. Lett. Assoc. 2006, 79, 39001. [CrossRef]

113. Santos, M.A.; Menetti, M.; Alcanis, J.; Brito, F.A.; Silva, R. CMB constraints on $\beta$-exponential inflationary models. arXiv 2017, arXiv:1710.09808.

114. Freese, K.; Frieman, J.A.; Olinto, A.V. Natural inflation with pseudo Nambu-Goldstone bosons. Phys. Rev. Lett. 1990, 65, 3233. [CrossRef] [PubMed]

115. Freese, K.; Kinney, W.H. On Natural Inflation. Phys. Rev. D 2004, 70, 083512.

116. Freese, K.; Kinney, W.H. Natural Inflation: Consistency with Cosmic Microwave Background Observations of Planck and BICEP 2. J. Cosmol. Astropart. Phys. 2015, 2015, 044. [CrossRef]

117. Kohri, K.; Lim, C.S.; Lin, C.M. Distinguishing between Extra Natural Inflation and Natural Inflation after BICEP2. J. Cosmol. Astropart. Phys. 2014, 2014, 001. [CrossRef]

118. Márián, I.G.; Defenu, N.; Trombettoni, A.; Nándori, I. Pseudo Periodic Higgs Inflation. arXiv 2017, arXiv:1705.10276.

119. Ross, G.G.; Germán, G. Hybrid natural inflation from non Abelian discrete symmetry. Phys. Lett. B 2010, 684, 199-204. [CrossRef]

120. Hebecker, A.; Kraus, S.C.; Westphal, A. Evading the Lyth bound in Hybrid Natural Inflation. Phys. Rev. D 2013, 88, 123506. [CrossRef]

121. Vázquez, J.A.; Carrillo-González, M.; Germán, G.; Herrera-Aguilar, A.; Hidalgo, J.C. Constraining Hybrid Natural Inflation with recent CMB data. J. Cosmol. Astropart. Phys. 2015, 2015, 039. [CrossRef]

122. Ross, G.G.; Germán, G.; Vázquez, J.A. Hybrid Natural Inflation. J. High Energy Phys. 2016, $2016,10$. [CrossRef]

123. Ross, G.G.; Germán, G. Hybrid Natural Low Scale Inflation. Phys. Lett. B 2010, 691, 117-120. [CrossRef]

124. Hebecker, A.; Kraus, S.C.; Witkowski, L.T. D7-Brane Chaotic Inflation. Phys. Lett. B 2014, 737, 16-22. [CrossRef]

125. Bezrukov, F.; Shaposhnikov, M. The standard model Higgs boson as the Inflaton. Phys. Lett. B 2008, 659, 703-706.

126. Bezrukov, F. The Higgs field as an inflaton. Class. Quantum Gravity 2013, 30, 214001. [CrossRef]

127. Gorbunov, D.; Tokareva, A. $\mathrm{R}^{2}$-inflation with conformal SM Higgs field. J. Cosmol. Astropart. Phys. 2013, 2013, 021. [CrossRef]

128. Zeynizadeh, S.; Akbarieh, A.R. Higgs inflation and general initial conditions. Eur. Phys. J. C 2015, 75, 355. [CrossRef]

129. Rubio, J. Higgs inflation and vacuum stability. J. Phys. Conf. Ser. 2015, 631, 012032. [CrossRef]

130. Sebastiani, L.; Cognola, G.; Myrzakulov, R.; Odintsov, S.D.; Zerbini, S. Nearly Starobinsky inflation from modified gravity. Phys. Rev. D 2014, 89, 023518. [CrossRef]

131. Cook, J.L.; Dimastrogiovanni, E.; Easson, D.; Krauss, L.M. Reheating predictions in single field inflation. J. Cosmol. Astropart. Phys. 2015, 2015, 047. [CrossRef]

132. Kallosh, R.; Linde, A.; Roest, D. Large Field Inflation and Double $\alpha$-Attractors. J. High Energy Phys. 2014, 2014, 52. [CrossRef]

133. Anchordoqui, L.A.; Barger, V.; Goldberg, H.; Huang, X.; Marfatia, D. S-dual inflation: BICEP 2 data without unlikeliness. Phys. Lett. B 2014, 734, 134-136. [CrossRef]

134. Agarwal, A.; Myrzakulov, R.; Sami, M.; Singh, N.K. Quintessential inflation in a thawing realization. Phys. Lett. B 2017, 770, 200-208. [CrossRef]

135. Basilakos, S.; Barrow, J.D. Hyperbolic inflation in the light of Planck 2013. Phys. Rev. D 2015, 91, 103517. [CrossRef] 
136. Kallosh, R.A.; Linde, A. Planck, LHC, and $\alpha$-attractors. Phys. Rev. D 2015, 91, 083528. [CrossRef]

137. Gong, J.O.; Shin, C.S. Natural Cliff Inflation. arXiv 2017, arXiv:1711.08270.

138. Kallosh, R.A.; Linde, A. Escher in the Sky. Comptes Rendus Phys. 2015, 16, 914-927. [CrossRef]

139. Coleman, S.; Weinberg, E. Radiative Corrections as the Origin of Spontaneous Symmetry Breaking. Phys. Rev. D 1973, 7, 1888-1910. [CrossRef]

140. Barenboim, G.; Chun, E.J.; Lee, H.M. Coleman-Weinberg Inflation in light of Planck. Phys. Lett. B 2014, 730, 81-88. [CrossRef]

141. Iso, S.; Kohri, K.; Shimada, K. Small Field Coleman-Weinberg Inflation driven by Fermion Condensate. Phys. Rev. D 2015, 91, 044006. [CrossRef]

142. Conlon, J.P.; Quevedo, F. Kähler Moduli Inflation. J. High Energy Phys. 2006, 2006, 146. [CrossRef]

143. Linde, A.D. Hybrid inflation. Phys. Rev. D 1994, 49, 748-754. [CrossRef]

144. Maartens, R.; Wands, D.; Bassett, B.A.; Heard, I. Chaotic inflation on the brane. Phys. Rev. D 2000, 62, 041301. [CrossRef]

145. Galcagni, G. Slow roll parameters in braneworld cosmologies. Phys. Rev. D. 2004, 69, 103508. [CrossRef]

146. Bennai, M.; Chakir, H.; Sakhi, Z. On Inflation Potentials in Randall-Sundrum Braneworld Model. Electron. J. Theor. Phys. 2006, 9, 84-93.

147. Naciri, M.; Safsati, A.; Zarrouki, R.; Bennai, M. MSSM Braneworld Inflation. Adv. Stud. Theor. Phys. 2014, 8, 277-283. [CrossRef]

148. Okada, N.; Okada, S. Simple brane-world inflationary models in light of BICEP2. arXiv 2014, arXiv:1407.3544.

149. Maartens, R.; Koyama, K. Brane-World Gravity. Living Rev. Relativ. 2004, 13, 5. [CrossRef] [PubMed]

150. Choudhury, S. Can Effective Field Theory of inflation generate large tensor-to-scalar ratio within Randall Sundrum single braneworld? Nucl. Phys. B 2015, 894, 29-55. [CrossRef]

151. Santos, J.R.L.; Moraes, P.H.R.S. Fast-roll solutions from two scalar field inflation. arXiv 2015, arXiv:1504.07204.

152. Grøn, Ø. A new standard model of the universe. Eur. J. Phys. 2002, 23, 135-144. [CrossRef]

153. Covi, L.; Lyth, D.H.; Melchiorri, A. New constraints on the running-mass inflation model. Phys. Rev. D 2003, 67, 043507. [CrossRef]

154. Covi, L.; Lyth, D.H.; Melchiorri, A.; Odman, C.J. Running-mass inflation model and WMAP. Phys. Rev. D 2004, 70, 123521. [CrossRef]

155. Armendáriz-Picón, C.; Damour, T.; Mukhanov, V.F. k-inflation. Phys. Lett. B 1999, 458, 209-218. [CrossRef]

156. Garriga, J.; Mukhanov, V.F. Perturbations in k-inflation. Phys. Lett. B 1999, 458, 219-225. [CrossRef]

157. Li, S.; Liddle, A.R. Observational constraints on tachyon and DBI inflation. J. Cosmol. Astropart. Phys. 2014, 2014, 044. [CrossRef]

158. Lorenz, L.; Martin, J.; Ringeval, C. K-inationary Power Spectra in the Uniform Approximation. Phys. Rev. D 2008, 78, 083513. [CrossRef]

159. Tsujikawa, S. Distinguishing between inflationary models from cosmic microwave background. Prog. Theor. Exp. Phys. 2014, 2014, 06B104. [CrossRef]

160. Guo, R.Y.; Zhang, X. Constraints on inflation revisited: An analysis including the latest local measurement of Hubble constant. Eur. Phys. J. C 2017, 77, 882. [CrossRef]

161. Hebecker, A.; Kraus, S.C.; Lüst, D.; Steinfurt, S.; Weigand, T. Fluxbrane inflation. Nucl. Phys. B 2012, 854, 509-551. [CrossRef]

162. Pal, B.K.; Pal, S.; Basu, B. Mutated Hilltop Inflation: A Natural Choice for Early Universe. J. Cosmol. Astropart. Phys. 2010, 2010, 029. [CrossRef]

163. Pal, B.K. Mutated hilltop inflation revisited. arXiv 2017, arXiv:1711.00833.

164. Eshagli, M.; Zarei, M.; Riazi, N.; Riasatpour, A. A non-minimally coupled potential for inflation and dark energy after Planck 2015: A Comprehensive Study. J. Cosmol. Astropart. Phys. 2015, 2015, 037. [CrossRef]

165. Maity, D. Minimal Higgs inflation. Nucl. Phys. B 2017, 919, 560-568. [CrossRef]

166. Hossain, Md.W.; Myrzakulov, R.; Samu, M.; Saridakis, E.N. B mode polarization á la BICEP2 and relic gravity waves produced during quintessential inflation. Phys. Rev. D 2014, 89, 123513. [CrossRef]

167. Bruck, C.; Dimopoulos, K.; Longden, C.; Owen, C. Gauss-Bonnet-coupled Quintessential Inflation. arXiv 2017, arXiv:1707.06839.

168. Dinda, B.R.; Kumar, S.; Sen, A.A. Inflationary generalized Chaplygin gas and dark energy in the light of the Planck and BICEP2 experiments. Phys. Rev. D 2014, 90, 083515. [CrossRef] 
169. Kobayashi, T.; Seto, O.; Yamaguchi, Y. Axion monodromy inflation with sinusoidal corrections. Prog. Theor. Exp. Phys. 2014, 2014, 103E01. [CrossRef]

170. Barrow, J.D. Graduated inflationary universes. Phys. Lett. B 1990, 235, 40-43. [CrossRef]

171. Barrow, J.D.; Saich, P. The behaviour of intermediate inflationary universes. Phys. Lett. B 1990, $249,406-410$. [CrossRef]

172. Mohammadi, A.; Ossoulian, Z.; Golanbari, T.; Saaidi, K. Intermediate inflation with modified kinetic term. Astrophys. Space Sci. 2015, 359, 7. [CrossRef]

173. Rezazadeh, K.; Karami, K.; Karimi, P. Intermediate inflation from a non-canonical scalar field. J. Cosmol. Astropart. Phys. 2015, 2015, 053. [CrossRef]

174. Nazavari, N.; Mohammadi, Z.; Saaidi, K. Intermediate inflation driven by DBI scalar field. Phys. Rev. D 2016, 93, 123504. [CrossRef]

175. Campo, S.; Herrera, R. Intermediate inflation on the brane. Phys. Lett. B 2009, 670, 266-270. [CrossRef]

176. Motohashi, H.; Starobinsky, A. Constant roll inflation: Confrontation with recent observational data. Europhys. Lett. 2017, 117, 39001. [CrossRef]

177. Cicciarella, F.; Mabillard, J.; Pieroni, M. New perspectives on constant-roll inflation. arXiv 2017, arXiv:1709.03527.

178. Karam, A.; Marzola, L.; Pappas, T.; Racioppi, A.; and Tamvakis, K. Constant-Roll (Quasi-) Linear Inflation. arXiv 2017, arXiv:1711.09861.

179. Berera, A. Warm Inflation. Phys. Rev. Lett. 1995, 75, 3218-3221. [CrossRef] [PubMed]

180. Berera, A.; Moss, I.G.; Ramos, R.O. Warm Inflation and its Microphysical Basis. Rep. Prog. Phys. 2009, 72, 026901. [CrossRef]

181. Campo, S. Warm Inflationary Universe Models. In Aspects of Today's Cosmology; Alfonso-Faus, A., Ed.; InTech: Rijeka, Croatia, 2011; ISBN 978-953-307-626-3.

182. Bartrum, S.; Bastero-Gil, M.; Berera, A.; Cerezo, R.; Ramos, R.O.; Rosa, J.G. The importance of being warm (during inflation). Phys. Lett. B 2014, 732, 116-121. [CrossRef]

183. Panotopoulos, G.; Videla, N. Warm $(\lambda / 4) / \varphi^{4}$ inflationary universe model in light of Planck 2015 results. Eur. Phys. J. C 2015, 75, 525. [CrossRef]

184. Grøn, Ø. Warm Inflation. Universe 2016, 2, 20. [CrossRef]

185. Bastero-Gil, M.; Bhattacharya, S.; Dutta, K.; Gangopadhyay, R.M. Constraining Warm Inflation with CMB data. arXiv 2017, arXiv:1710.10008.

186. Arya, R.; Dasgupta, A.; Goswami, G.; Prasad, J.; Rangarajan, R. Revisiting CMB constraints on Warm Inflation. arXiv 2017, arXiv:1710.11109.

187. Bastero-Gil, M.; Berera, A. Warm Inflation model building. Int. J. Mod. Phys. A 2009, 24, $2207-2240$. [CrossRef]

188. Hall, L.; Moss, I.G.; Berera, A. Constraining warm inflation with the cosmic microwave background. Phys. Lett. B 2004, 589, 1-6. [CrossRef]

189. Visinelli, L. Natural Warm Inflation. J. Cosmol. Astropart. Phys. 2011, 2011, 013. [CrossRef]

190. Hall, L.; Moss, I.G.; Berera, A. Scalar perturbation spectra from warm inflation. Phys. Rev. D 2004, 69, 083525. [CrossRef]

191. Moss, I.G.; Xiong, C. On the consistency of warm inflation. J. Cosmol. Astropart. Phys. 2008, $2008,023$. [CrossRef]

192. Herrera, R. Reconstructing warm inflation. arXiv 2018, arXiv:1801.05138.

193. Visinelli, L. Observational constraints on Monomial Warm Inflation. J. Cosmol. Astropart. Phys. 2016, 2016, 054. [CrossRef]

194. Sharif, M.; Saleem, R. Warm Anisotropic Inflationary Universe Model. Eur. Phys. J. C 2014, 74, 2738. [CrossRef]

195. Taylor, A.N.; Berera, A. Perturbation Spectra in the Warm Inflationary Scenario. Phys. Rev. D 2000, 62, 083517. [CrossRef]

196. Campo, S.; del Herrera, R.; Pavón, D. Cosmological perturbations in warm inflationary models with viscous pressure. Phys. Rev. D 2007, 75, 083518. [CrossRef]

197. Setare, M.R.; Kamali, M. Warm-intermediate inflationary model with viscous pressure in high dissipative regime. Gen. Relativ. Gravit. 2014, 46, 1698. [CrossRef]

198. Fairbairn, M.; Tytgat, M.H.G. Inflation from a Tachyon Fluid? Phys. Lett. B 2002, 546, 1-7. [CrossRef] 
199. Li, X.; Liu, D.; Hao, J. On tachyon inflation. J. Shanghai Norm. Univ. 2004, 33, 29.

200. Kofman, L.; Linde, A. Problems with Tachyon Inflation. J. High Energy Phys. 2002, 2002, 004. [CrossRef]

201. Barbosa-Cendejas, N.; De-Santiago, J.; German, G.; Hidalgo, J.C.; Mora-Luna, R.R. Theoretical and observationao constraints on Tachyon inflation. arXiv 2017, arXiv:1711.06693.

202. Gibbons, G.W. Cosmological evolution of the rolling tachyon. Phys. Lett. B 2002, 537, 1-4. [CrossRef]

203. Campo, S.; Herrera, R.; Toloza, A. Tachyon field in intermediate inflation. Phys. Rev. D 2009, $79,083507$. [CrossRef]

204. Kamali, V.; Basilakos, S.; Mehrabi, A. Tachyon warm-intermediate inflation in the light of Planck data. Eur. Phys. J. C 2016, 76, 525. [CrossRef]

205. Rashidi, N.; Nozari, K. Observational Status of Tachyon Matural Inflation and Reheating. arXiv 2017, arXiv:1712.05120.

206. Hamada, Y.; Kawai, H.; Nakanishi, Y.; Oda, K. Higgs inflation puts lower and upper bounds on tensor-to-scalar ratio and on Higgs-portal-dark-matter mass. arXiv 2017, arXiv:1709.09350.

207. Yi, Z.; Gong, Y. On the constant-roll inflation. arXiv 2017, arXiv:1712.07478.

208. Chervon, S.V. Inflationary Cosmological Models without Restrictions on a Scalar Field Potential. Gen. Relativ. Gravit. 2004, 36, 1547-1553. [CrossRef]

209. Chervon, S.V.; Novello, M.; Triay, R. Exact Cosmology and Specification of an Inflationary Scenario. Gravit. Cosmol. 2005, 11, 329-332.

210. Chervon, S.V.; Fomin, I.V. On Calculation of the Cosmological Parameters in Exact Models of Inflation. Gravit. Cosmol. 2008, 14, 163-167. [CrossRef]

211. Ellis, J.; Wands, D. Inflation. In Review of Particle Physics. Chin. Phys. C 2016, 40, 367-378.

(C) 2018 by the author. Licensee MDPI, Basel, Switzerland. This article is an open access article distributed under the terms and conditions of the Creative Commons Attribution (CC BY) license (http://creativecommons.org/licenses/by/4.0/). 